\title{
PROTEOMIC ANALYSIS OF AGE-RELATED CHANGES IN OVINE CEREBROSPINAL FLUID
}

Carl PC Chen, ${ }^{1,2}$ Jane E Preston, ${ }^{1}$ Shaobo Zhou, ${ }^{3}$ Heidi R Fuller, ${ }^{4,5}$ David G A Morgan, ${ }^{5,6}$ Ruoli Chen ${ }^{\mathbf{1 , 5 , 6}}$

${ }^{1}$ Institute of Pharmaceutical Science, King's College London, London, SE1 7UL UK

${ }^{2}$ Department of Physical Medicine and Rehabilitation, Chang Gung Memorial

Hospital at Linkou, College of Medicine, Chang Gung University, Kwei Shan, Taoyuan County, Taiwan, ROC

${ }^{3}$ Institute of Biological and Environmental Science and Technology (iBEST),

School of Life Sciences, University of Bedfordshire, Luton LU1 3JU, UK

${ }^{4}$ Wolfson Centre for Inherited Neuromuscular Disease, RJAH Orthopaedic Hospital, Oswestry, SY10 7AG, UK.

${ }^{5}$ Institute for Science and Technology in Medicine (ISTM), Keele University, Staffordshire ST5 5BG, UK

${ }^{6}$ School of Pharmacy, Keele University, Staffordshire ST5 5BG, UK

\section{Correspondence}

Dr Ruoli Chen

School of Pharmacy, Institution for Science and Technology in Medicine, Keele University, Staffordshire ST5 5BG, UK

Email: r.chen@keele.ac.uk 


\begin{abstract}
Cerebrospinal fluid (CSF) circulates through the brain and has a unique composition reflecting the biological processes of the brain. Identifying ageing CSF biomarkers can aid in understanding the ageing process and interpreting CSF protein changes in neurodegenerative diseases. In this study, ovine CSF proteins from young (1-2 year old), middle aged (3-6 year old) and old (7-10 year old) sheep were systemically studied. CSF proteins were labelled with iTRAQ tagging reagents and fractionated by 2-dimensional high performance, liquid chromatography. Tryptic peptides were identified using MS/MS fragmentation ions for sequencing and quantified from iTRAQ reporter ion intensities at m/z 114, 115, 116 and 117 . Two hundred thirty one peptides were detected, from which 143 proteins were identified. There were 52 proteins with $>25 \%$ increase in concentrations in the old sheep compared to the young. 33 of them increased $>25 \%$ but $<50 \%, 13$ increased $>50 \%$ but $<1$ fold, 6 increased $>1$ fold [i.e. haptoglobin $(\mathrm{Hp})$, haemoglobin, neuroendocrine protein 7B2, IgM, fibrous sheath interacting protein 1, vimentin]. There were 18 proteins with $>25 \%$ decrease in concentrations in the old sheep compared to the young. 17 of them decreased $>25 \%$ but $<50 \%$, and histone deacetylase 7 (HDAC7) was gradually decreased for over $80 \%$. Glutathione S-transferase was decreased in middle aged CSF compared to both young and old CSF. The differential expressions of 3 proteins (Hp, neuroendocrine protein 7B2, IgM) were confirmed by immunoassays. These data expand our current knowledge regarding ovine CSF proteins, supply the necessary information to understand the ageing process in the brain and provide a basis for diagnosis of neurodegenerative diseases.
\end{abstract}




\section{Highlights}

- CSF protein changes during normal ageing process

- Seventy proteins were changed in concentration among different aged sheep CSF

- A common ageing CSF biomarker identified to date is immunoproteins

- Identifying ageing CSF biomarker help understand the ageing process in the brain

Key words: ageing, CSF, biomarkers, proteomics, choroid plexus, neurodegenerative disease 


\section{INTRODUCTION}

Cerebrospinal fluid (CSF) is a clear, colourless bodily fluid that circulates through the brain and communicates freely with the brain extracellular fluid. In adult humans, about two-thirds of CSF is secreted by the choroid plexus (CP) at the rate of about $0.35-0.4 \mathrm{ml} / \mathrm{min}$ or $500-600 \mathrm{ml} /$ day, while the remainder is from the extrachoroidal sources [1]. CSF has a unique composition that is different from the plasma, due to the presence of blood-brain barrier (BBB) and blood-CSF barrier (BCSFB), the active secretion at the $\mathrm{CP}$ and specific transport systems for moving substances between blood and CSF. In comparison to plasma ultrafiltrate, CSF contains higher concentrations of sodium, chloride, and magnesium and lower concentrations of glucose, proteins, amino acids, uric acid, potassium, bicarbonate, calcium and phosphate [2]. The CSF is in direct contact with the brain interstitial fluid, and the composition of the CSF therefore reflects biological processes occurring in the brain [3]. This understanding has led to an interest in the potential for discovering biomarkers within the CSF, which can be used to monitor brain function and aid in diagnosis of neurological diseases.

During ageing, several structural, chemical, and functional changes occur in the brain. The weight and volume of both cerebral cortex and hippocampus reduce, and the ventricular system expands to fill the space vacated by the brain parenchyma [4]. In contrast, the $\mathrm{CP}$ in the lateral ventricles doubles in weight, leading to significant increase of the ratio of the $\mathrm{CP}$ and the brain in weight $[5,6]$. The CSF secretion by the $\mathrm{CP}$ is decreased, leading to a decreased overall turnover of CSF which can affect its protein composition. 
There are few studies on the CSF biology during ageing in absence of any neurological diseases, and the drastically incomplete knowledge hinders us from understanding the ageing process in the brain. In this study, we systemically characterized age-related protein changes in ovine CSF using a gel free proteomic mass spectrometry (MS) approach with isobaric labelled samples (iTRAQ) techniques. The advantage of using sheep in this study is that adequate CSF samples of all age groups can be obtained, with facile control over gender selection and environmental factors. Furthermore, we have previous characterized the age-related changes in the $\mathrm{CP}$ structure and function in the sheep, and found that the secretion rate of the CP is decreased thus slowed overall CSF turnover, which contributed to the increased protein concentrations within the CSF with increasing age [6-10]. 


\section{METHODS}

\subsection{Sample collection}

Clun Forest strain adult female sheep aged between 1 and 10 year old were divided into 3 groups: young (aged 1-2 years); middle-aged (3-6 years) and old (7-10 years). Each group comprises 7 sheep. Sheep were anaesthetized with i.v. thiopentone sodium (20 mg.kg $\left.{ }^{-1}\right)$ and heparinised (20,000 IU heparin $\left.\mathrm{kg}^{-1}\right)$. CSF samples were collected from the cisterna magna by needle puncture [11]. Samples were spun at $10000 \mathrm{~g}$ for 10 min at $4{ }^{\circ} \mathrm{C}$. CSF samples with any blood contamination determined by the presence of erythrocytes in the solution or precipitation were discarded. Supernatants were immediately stored at $-80{ }^{\circ} \mathrm{C}$ until being analysed. All procedures were within the Home Office Scientific procedures Act, 1986 (HMSO, London, UK), and were approved by King's College London research ethics committee.

\subsection{Sample preparation for MS analysis}

Equal volumes of CSF samples from seven individuals within each group were pooled to give a final volume of $80 \mu \mathrm{l}$ per age group. An internal standard (IS) was prepared by pooling equal amount of each sample from the analysis set. The pooled samples were then digested with sequencing grade porcine trypsin (Promega, Fitchburg, Wisconsin, USA) overnight at $37{ }^{\circ} \mathrm{C}$, followed by reduction and alkylation steps performed according to the instructions outlined in the iTRAQ labelling kit (AB Sciex, Framingham, MA, USA). Following this, the digests were then dried down in a vacuum centrifuge and iTRAQ labelling carried out also according to instructions in the iTRAQ labelling kit. Each iTRAQ tag was assigned as follows: iTRAQ reagent 114 for Young, 115 for Middle-age, 116 for Old and 117 for the IS. 
Both MS and MS/MS analysis was performed on the fractionated peptides using an Applied Biosystems 4800 MALDI TOF/TOF mass spectrometer. The mass spectrometer was operated under control of 4000 Series Explorer v3.5.2 software (Applied Biosystems, Waltham, Massachusetts, USA). A total of 1000 shots per MS spectrum (no stop conditions) and 2500 shots per MS/MS spectrum (no stop conditions) were acquired. The following MS/MS acquisition settings were used: $2 \mathrm{KV}$ operating mode with $\mathrm{CID}$ on and precursor mass window resolution set to 300.00 (FWHM). Peak lists of MS and MS/MS spectra were generated using 4000 Series Explorer v3.5.2 software and the following parameters were used after selective labelling of monoisotopic mass peaks: MS peak lists: $\mathrm{S} / \mathrm{N}$ threshold 10 , Savitzky Golay smoothing (3 points across peak (FWHM)), no baseline correction, MS/MS peak lists: S/N threshold 14; smoothing algorithm: Savitzky Golay, smoothing (7 points across peak (FWHM)).

\subsection{Quantitative MS analysis}

Liquid chromatography fractionation and subsequent MS analysis was conducted as described before [12]. Briefly, labelled tryptic peptides obtained from protein digestion were pooled and dissolved in $2.4 \mathrm{ml}$ of SCX buffer A (10 mM phosphate, $20 \%$ acetonitrile) and centrifuged at $15,000 \times g$ for 5 min to remove any insoluble debris. The peptide mixture was loaded onto a Polysulphoethyl A column $(4.6 \mathrm{~mm} \times$ $100 \mathrm{~mm}, 5 \mu \mathrm{m}, 300 \AA$, PolyLC Inc.) using a flow rate of $800 \mu \mathrm{l} / \mathrm{min}$. The bound sample was washed using SCX buffer A for approximately 20 min, until non-binding mixture components were removed (as determined by the UV traces returning to baseline levels). Peptides were then eluted at $400 \mu \mathrm{l} / \mathrm{min}$ using a gradient of SCX 
buffer B (SCX A containing $1 \mathrm{M}$ potassium chloride) of $0-30 \%$ over $30 \mathrm{~min}$. Fractions were collected manually at $60 \mathrm{~s}$ intervals. SCX fractions containing peptides were dried to completeness and dissolved in $30 \mu \mathrm{l}$ of RP buffer A ( $2 \%$ acetonitrile) before separating further using an Ultimate 3000 chromatography system connected to a Probot fraction collector (Dionex, Sunnyvale, CA, USA). Samples were randomised and loaded onto a C18 trapping column before eluting onto a $75 \mu \mathrm{m}$ internal diameter C18 PepMap column. Peptides were washed for 15 min in RP buffer A before eluting with a $2-50 \%$ gradient of acetonitrile over $120 \mathrm{~min}$, followed by further elution with $90 \%$ acetonitrile for $15 \mathrm{~min}$. Eluted peptide fractions were spotted onto a MALDI-MS target plate every $10 \mathrm{~s}$ with $3 \mathrm{mg} / \mathrm{ml} \alpha$-cyano-2-hydroxy cinnamic acid at a flow rate of $1.2 \mu \mathrm{l} / \mathrm{min}$. A blank injection was performed between peptide runs to minimise sample carry-over.

Peptides were analysed using an Applied Biosystems 4800 Proteomics Analyser with close external standards to calibrate the instrument. Survey spectra were collected from the range 900 to $4000 \mathrm{Da}$, with a focus mass of $2500 \mathrm{Da}$. Fifty shots were fired per sub-spectrum, with 1000 total shots per spectrum without stop conditions. All subspectra were accepted, and laser intensity was set to 3750 . For product ion analysis, a maximum of 10 precursor ions per spot were selected automatically with a minimum signal-to-noise ratio of 40. Lowest-intensity precursors were acquired first.

An automated database search was run using GPS Explorer v3.6 (AB Sciex, Framingham, MA, USA). MASCOT was used as the search engine to search the NCBI non-redundant database version Oct 04, 2011, using the following search parameters: species $=$ mammals; precursor ion mass tolerance of $150 \mathrm{ppm}$; MS/MS 
fragment ion mass tolerance of $0.3 \mathrm{Da}$; iTRAQ fragment ion mass tolerance of 0.2 Da. Oxidation of methionine residues were allowed as variable modifications, and Nterm (iTRAQ), lysine (iTRAQ) and MMTS modification of cysteine residues were set as fixed modifications. Protein were identified on the basis of having at least one peptide with an ion score above $95 \%$ confidence. All identified CSF proteins were subjected to functional analyses using PubMed search (https://www.ncbi.nlm.nih.gov/pubmed/) and the PANTHER classification system (http://www.pantherdb.org/genes/batchIdSearch.jsp) [13]. Only peptides unique for a given protein was considered for relative quantification. iTRAQ Ratios were normalized using the following formula: iTRAQ Ratio = Ratio/(median iTRAQ Ratio of all found pairs) that was applied in GPS Explorer software.

\subsection{Enzyme-linked immunosorbent assay (ELISA)}

Experiments were performed using commercially available ovine protein ELISA kits: sheep haptoglobin (HP), sheep neuroendocrine protein 7B2 (MyBioscience, San Diego, CA, USA), according to the manufactory instructions. The target protein concentrations in CSF were determined according to the standard curve with known concentrations of the target proteins.

\subsection{Western Immunoblotting}

CSF samples $(15 \mu \mathrm{l})$ were mixed with equal amount of $2 \mathrm{x}$ Laemmli sample buffer (Sigma-Aldrich, St. Louis, Missouri, USA). The samples were loaded onto precast 10\% SDS-polyacrylamide gel (Bio-Rad Laboratories, Hercules, CA, USA) and were electrophoresed at $60 \mathrm{mV}$ constant voltage until the dye front reached the bottom of the gel. The electrophoresed protein was transferred onto a $0.45 \mu \mathrm{m}$ thick 
nitrocellulose membrane, which was saturated for $1 \mathrm{~h}$ with $5 \%(\mathrm{w} / \mathrm{v})$ milk powder in tris-buffered saline (TBS) containing 0.05\% Tween 20 (TBS-T). Membranes were then incubated with the primary antibodies rabbit anti-sheep mu chain (1:500)(SigmaAldrich, St. Louis, Missouri, USA), anti-sheep IgG (1:500)(Zymed-Thermo Fisher Scientific, Waltham, Massachusetts, USA); or anti-human haemoglobin $\beta / \gamma / \delta(\mathrm{H}-76)$ (1:200)(Santa Cruz Biotechnology, Santa Cruz, CA, USA) overnight at $4{ }^{\circ} \mathrm{C}$. Thereafter the membranes were washed and incubated with horseradish peroxidaseconjugated goat anti-rabbit IgG (1:1000)(Dako-Agilent technologies, Santa Clara, California, USA). After final wash, the immunostained proteins were developed using a Pierce ECL developing kit (Thermo Fisher Scientific, Waltham, Massachusetts, USA), and were detected by a Fluorchem M image system (ProteinSimple, San Jose, California, USA). A CSF sample was used as an internal standard for each Western blotting experiment. The Western bolts images were analysed using Image studio lite version 5.2 software (LI-COR Biosciences, Lincoln, Nebraska, USA). Each band was analysed by manually selecting them to obtain the density measurement. The ratios of the sample density to the internal reference were used and compared among age groups.

\subsection{Data Analysis}

All values were expressed as mean \pm SEM. One way ANOVA with Tukey post-hoc study was used to analyse comparisons among the 3 age groups. SPSS version 19 (SPSS INC., Chicago, USA) was used for the analysis. Values of $\mathrm{P}<0.05$ were considered statistically significant, and values of $\mathrm{P}<0.01$ were considered statistically highly significant. 


\section{RESULTS}

\subsection{Identification of proteins in CSF}

The total protein concentration in the CSF increased with advancing age, as these sheep showed age-related reduction in CSF secretion / turnover [10]. We therefore applied equal amount volume of CSF in the analysis instead of using equal amount of CSF proteins. Two hundred and thirty one peptides were detected from the pooled CSF samples. All but 5 of the peptides were named. From these peptides, 143 proteins were identified. Of these proteins, 78 were identified with at least two peptides while the reminder was identified with a single peptide (Supplement 1 and 2). These proteins included not only relatively abundant proteins, such as albumin, IgG, transthyretin, transferrin, haptoglobin, complements, and various apolipoproteins, but also lower abundance peptides such as neuropeptide Y (see Supplement 1 and 2). The functional classification of the 143 identified proteins was listed in Table 1.

\subsection{Quantitative analysis of CSF proteins in different age groups}

iTRAQ not only identifies CSF proteins but also enable us to quantitatively compare the proteins with different labels [14]. Although the protein profile in each iTRAQ experiment may vary when complex protein mixture are analysed, quantitative comparison is valid when the same peptide is detected in samples at the same time. We therefore compared the ratios of an individual age group to the pooled CSF group. Our results demonstrated that there were substantial quantitative differences in many proteins between the three groups. We separated these proteins into 2 main groups: whose levels have increased (Table 2) or decreased (Table 3) greater than 25\%. There were 52 proteins with $>25 \%$ increase in concentrations in the old sheep compared to the young (Table 2). 33 of them increased $>25 \%$ but $<50 \%$ [e.g. insulin like growth 
factor binding proteins (IGFBPs), Complements], 13 increased $>50 \%$ but $<1$ fold [e.g. transthyretin (TTR), apolipoprotein C-III), 6 increased $>1$ fold [i.e. haptoglobin (Hp), haemoglobin (HGB), neuroendocrine protein 7B2, immunoglobin $\mathrm{M}(\operatorname{IgM})$, fibrous sheath interacting protein 1 , vimentin] (Table 2 ). There were 18 proteins with $>25 \%$ decrease in concentrations in the old sheep compared to the young. 17 of them decreased $>25 \%$ but $<50 \%$ (e.g. fibrinogen, transferrin), and histone deacetylase 7 (HDAC7) was gradually decreased for over $80 \%$ (Table 3). Glutathione S-transferase was decreased in middle aged CSF compared to both young and old CSF.

\subsection{Validation of proteins associated with ageing process}

To confirm peptide-based protein identifications and variant proteins as suggested by tandem MS, ELISA and Western Immunoblotting were performed on the CSF samples. As the first step towards verifying these ageing biomarkers in Table 2, that displayed more than 1 fold among the 3 age groups, all commercially available antibodies and ELISA kits for the sheep proteins were purchased for further analysis. These were sheep Hp ELISA kit, sheep neuroendocrine protein 7B2 ELISA kit, antisheep mu chain and sheep IgG antibodies. In addition, an antibody for human haemoglobin $\beta / \gamma / \delta$ (H-76) was bought as there were no specific antibodies for sheep haemoglobin. Specific ovine protein ELISA kits indicated there were significant increase of Hp and neuroendocrine protein 7B2 in the old CSF (Fig 1). Western blots of sheep mu chain showed significant increase in IgM in old CSF, in contrast, there were no changes in IgG among age groups (Fig 2). There were no bands on Western immunoblots with the anti-human haemoglobin $\beta / \gamma / \delta$ antibody on the ovine CSF samples (data not shown). 


\section{DISCUSSION}

In this study, we studied ovine CSF proteome and compared CSF protein levels between different ages. We identified 143 different ovine CSF proteins using MS/MS fragmentation ions for sequencing, and generated the most complete characterization of ovine CSF proteome to date according to our knowledge. The number of proteins detected in ovine CSF was similar with in bovine CSF [15], but was lower compared to human CSF proteome [16], probably because both the ovine and bovine protein database are far less than completed than the human [17]. A large percentage of ovine CSF proteins (21.7\%) are binding proteins, including the major CNS apolipoproteins (e.g. A, C, D, E), consistent with CSF "sink action" [1]. We did not find the apolipoprotein B, the major apolipoprotein in the blood that is not present in CSF, indicating the minimal blood contamination in our CSF samples $[18,19]$. To maximize efficient use of the MS, 7 ovine CSF samples with same age were pooled into a group, similar to our previous studies [12]. This approach will ensure adequate materials to identify low abundant proteins and minimized inter-subject variability, which was kept in minimum as the sheep was carefully controlled over their age, gender, disease states, season etc. The variability can be further addressed by performing careful follow-on / validation of aliquot from each individual animal.

Using iTRAQ reporter ion intensities at $\mathrm{m} / \mathrm{z} 114,115,116,117,52$ proteins were found to be increased for more than $25 \%$ between ages, and 6 of them (neuroendrocrine protein 7B2, IgM, fibrous sheath interacting protein 1, Hp, HGB, vimentin) were gradually increased for more than one fold with age. There were 18 proteins with $>25 \%$ decrease in concentrations between ages, and the HDAC7 
gradually decreased in concentrations for more than $80 \%$. GST decreased in middle aged CSF samples compared to both young and old CSF samples.

While high throughput proteomic analysis of CSF proteins selects candidate proteins for further study, candidate proteins identified by this approach need to be validated before their biological roles are pursued extensively. As the first step towards developing candidate ageing markers, we sought to use commercially available antibodies against the candidate proteins on individual ovine CSF samples. We used as much as possible of commercially available sheep protein diagnosis kits/antibodies, and validated 3 candidate proteins: $\mathrm{Hp}$, neuroendocrine secretory protein 7B2 and $\operatorname{IgM}$.

$\mathrm{Hp}$ is an acute-phase protein that scavenges HGB in the event of haemolysis, but also can be induced by inflammation [20, 21]. Human CSF Hp has a role in the protection of the CNS against autoimmune inflammatory responses after aneurysmal subarachnoid haemorrhage [22]. Increased human CSF Hp concentration were found in a number of neurological diseases, e.g. idiopathic normal pressure hydrocephalus, traumatic brain injury, Gullain-Barre syndrome and neuromyelitis optica [23, 24]. Chamoun et al. [25] reported there was an increased likelihood of detecting Hp in human CSF with age and suggested Hp was a marker of BCSFB dysfunction.

Neuroendocrine secretory protein $7 \mathrm{~B} 2$ resides in the secretory granules of neuroendocrine cells and functions as a specific chaperone for the proprotein convertase 2 [26] as well as an anti-aggregation secretory chaperone associated with neurodegenerative diseases [27]. The normal concentration of 7B2 in human CSF is 
$10-100$ folds greater than in plasma $[26,28]$, suggesting $7 \mathrm{~B} 2$ is originated from the brain.

The brain has historically been considered an 'immune privileged region', as it is separated from peripheral circulation by $\mathrm{BBB}$ and BCSFB. However, the brain also contains microglia, the counterpart to macrophages which actively surveys the brain [29]. A recent study suggests that brain immune surveillance communicates with the immune system and can generate adaptive immune responses [30]. In our study, 13.3\% identified CSF proteins were immunoproteins, which are proteins with immunological activities, e.g. immunoglubins and complements. Among them, IgM significant was increased in the old ovine CSF. IgM plays an important role in cytolytic reactions and agglutination [31], and is strongly related with a fatal neurodegenerative disease called prion disease [32]. There was a correlation between CSF IgM and CSF apolipoproteins in patients with neurological diseases [33]. In contrast, IgG levels in the CSF were not different between different age groups. IgM is the largest antibody and moves out of the brain only with CSF clearance, while IgG is the most abundant but the smallest antibody and moves out of the brain via additional efflux system [34]. The increase of IgM in the old CSF could be caused by either the old sheep were subjected to some infections that the young sheep were not, and / or the old sheep had slower CSF turnover rates compared to the young ones $[10]$.

The HGB expression in vertebrates was previously thought to be restricted to red blood cells, however the expression of HGB was found in both rat and human neurons $[35,36]$ and HGB was detected in the CSF of normal human controls [37]. HGB was 
involved in neuronal mitochondrial energetics with epigenetic changes to histones and may provide neuroprotection in multiple sclerosis by supporting neuronal metabolism [38]. Although the sequence homology of haemoglobin $\beta$ in ovis aries vs. human is $83 \%$ homology, Western blots with an anti-human haemoglobin $\beta / \gamma / \delta(\mathrm{H}-76)$ antibody showed no reaction with the ovine CSF samples.

To our best knowledge, there are only 2 studies on ageing CSF biomarkers in human. Utilising a shotgun proteomics strategy in conjunction with ICAT (isotope-coded affinity tags), Zhang et al. [18] identified 312 different CSF proteins, of which 187 were identified with at least two peptides while the reminder was identified with a single peptide. The authors discovered 6 proteins that were changed more than one fold between the young and old human CSF, i.e. agrin, bromodomain, splice isoform $\gamma$-2 of P36873, protein phosphatase, hypothetical protein, serine/threonine protein phosphatase, ubiquinol-cytochrome $c$ reductase. An important issue in Zhang's study is that the changes in the relative amount of proteins were measured with pooled CSF samples. The variability between humans is more than between sheep. The study could not define if the age-related difference found were due to difference in some individuals or being distributed over all participants. Although follow up immunoassays can partially address this problem, this approach is limited by availability of sufficiently sensitive antibodies. Another study on age-related changes in human CSF proteome was conducted by Baird et al. [19] using the Slow off-rate modified Aptamer Array technique. Baird et al. identified 82 proteins that were changed over age $28-82$, most of which are associated with immune system activation or response to injury. These biomarkers overlap with our discovery in immunoproteins, indicating a role for inflammatory process in CNS ageing. A recent 
study found in healthy ageing human CSF, there was a three-fold increase of soluble triggering receptor expressed on myeloid cell 2 (TREM 2), which functions in immune response and may be involved in chronic inflammation [39].

Ageing is associated with chronic inflammation. Inflammageing describes the close relationship between low-grade chronic inflammation and ageing in various organs, including the brain [40]. A number of theories have been developed to define the inflammageing, such as redox stress, mitochondrial damage, immunosenescence, endocrinosenescence, epigenetic modification etc [41]. The age-related changes in the brain including the inflammageing may provide a substrate for developing neurodegenerative disease. Indeed, inflammation has long been associated with neurodegenerative diseases [42]. Future studies on neurodegenerative diseases should consider the ageing factor. An important precondition for discovering CSF biomarkers in neurodegenerative diseases is an understanding of the dynamic nature of the CSF proteome during normal ageing process.

A number of Alzheimer's disease (AD) biomarkers, such as amyloid beta, tau, neurofilament light, were detected in ovine CSF. Total tau and neurofilament light levels were comparable with human levels [43]. We also found a few of AD related proteins in the ovine CSF, e.g. Apolipoprotein E, Amyloid beta A4 protein isoform $b$ precursor, amyloid precursor-like protein 1, and they were not significantly different in concentrations among the age groups. Sheep are not known to develop AD naturally, probably due to their shorter lifespan compared to humans and being culled once past their useful lifespan for the farmers [43]. 
In conclusion, this study has generated detail knowledge of the ovine proteome and presented a number of suitable candidate proteins for further study in terms of their physiological variation in CSF during ageing. It would be important to validate the results of this study in CSF from humans in future and to conduct further experiments to understand their roles in healthy ageing and neurodegerative diseases. Changes in the CSF proteins during ageing without disease may be small and heterogeneous between individuals. Thus a combination of a number of studies on CSF ageing biomarkers would provide a full picture of ageing related changes in CSF proteome. A common ageing CSF biomarker identified to date is immunoproteins indicating a role of the immune response and inflammation in normal ageing process in the brain. MS analysis usually serves to identify candidates, functional and follow up studies will be needed for understanding the physiology of brain ageing as well as for recognizing neurodegenerative disease-associated changes in CSF proteome patterns. 
COMPETING FINANCIAL INTERESTS

There is no conflict of interest.

\section{AUTHOR CONTRIBUTIONS}

This project was conceived by R.C. and J.E.P. The in vivo experiments in this paper were performed by C.P.C. and R.C., while the quantitative mass spectrometry experiment was conducted by H.R.F. Data were analysed and interpreted by R.C., H.R.F., and S.Z. The manuscript was prepared by R.C., S.Z., H.R.F., C.P.C, and D.G.A.M. All authors have read and approved the final copy.

\section{FUNDING}

This study was supported by research grants received from the Biotechnology and Biological Sciences Research Council (BBSRC) (BBD01381X1), the Wellcome Trust (200633/z/16/z), the Physiology Society (summer vacation studentship).

\section{ACKNOWLEDGMENTS}

We thank Ms Anam Baksh for performing some of the immunoassays. 


\section{REFERENCES}

[1]. Ghersi-Egea, J.F., Strazielle, N., Catala, M., Silva-Vargas, V., Doetsch, F., Engelhardt, B. (2018) Molecular anatomy and functions of the choroidal bloodcerebrospinal fluid barrier in health and disease. Acta Neuropathol., 135(3), 337-61.

[2]. Shen, D.D., Artru, A.A., Adkison, K.K. (2004) Principles and applicability of CSF sampling for the assessment of CNS drug delivery and pharmacodynamics. $A d v$ Drug Deliv Rev. 56(12), 1825-57.

[3]. Stoop, M.P., et al. (2010). Quantitative proteomics and metabolomics analysis of normal human cerebrospinal fluid samples._Mol Cell Proteomics. 9(9), 2063-75.

[4]. Wyss-Coray, T. (2016). Ageing, neurodegeneration and brain rejuvenation. Nature, 539(7628):180-186.

[5]. Spector, R., and Johanson, C.E. (1989). The mammalian choroid plexus. Sci. Am. $261,68-74$.

[6]. Chen, R.L., Preston, J.E. (2012). Changes in kinetic of amino acids uptake at the ageing ovine blood-cerebrospinal fluid barrier. Neurobiol. Ageing. 33, 121-133.

[7]. Chen, R.L., et al. (2009). Age-related changes in choroid plexus and bloodcerebrospinal fluid barrier function in the sheep. Exp. Geront. 44(4), 289-296.

[8]. Chen, R.L., Chen, P.C., Preston, J.E. (2010). Elevation of CSF albumin in old sheep: relations to CSF turnover and albumin extraction at blood CSF barrier. $J$ Neurochem. 113, 1230-1239.

[9]. Chen, C.P., Chen, R.L., Preston, J.E. (2010). The influence of cerebrospinal fluid turnover on age-related changes in cerebrospinal fluid protein concentrations. Neurosci Lett. 476(3), 138-141. 
[10]. Chen, C.P., Chen, R.L., Preston, J.E. (2012). The influence of ageing in the cerebrospinal fluid concentrations of proteins that are derived from the choroid plexus, brain and plasma. Exp Geront. 27(4), 323-328.

[11]. Chen, R.L., Sage, E.A., Dunn, M.J., Wait, R., Preston, J.E. (2006). Optimising ovine cerebrospinal fluid preparation for two-dimensional gel electrophoresis. Proteomics. 6(10), 3170-5.

[12]. Fuller, H.R., Man, N.T., Lam Le, T., Shamanin, V.A., Androphy, E.J., Morris, G.E. (2010). Valproate and bone loss: iTRAQ proteomics show that valproate reduces collagens and osteonectin in SMA cells. J Proteome Res. 9 (8), 4228-4233.

[13]. Mi, H., Muruganujan, A., Casagrande, J.T., Thomas PD. (2013). Large-scale gene function analysis with the PANTHER classification system. Nat Protoc. 8(8), $1551-1566$

[14]. Wiese, S., Reidegeld, K.A., Meyer, H.E., Warscheid, B. (2007). Protein labeling by iTRAQ: a new tool for quantitative mass spectrometry in proteome research. Proteomics. 7(3), 340-50.

[15]. Brenn, A., Karger, A., Skiba, M., Ziegler, U., Groschup, M.H. (2009). A comprehensive proteome map of bovine cerebrospinal fluid. Proteomics. 9(22), 51995205.

[16]. Schutzer, S.E., et al. (2012). Establishing the Proteome of Normal Human Cerebrospinal Fluid. PLoS ONE 5(6), e10980. doi:10.1371/journal.pone.0010980.

[17]. Chiaradia, E. et al. (2012). Proteomic evaluation of sheep serum proteins. BMC Vet Res. 8, 66. doi: 10.1186/1746-6148-8-66.

[18]. Zhang, J., et al. (2005). Quantitative proteomic analysis of age-related changes in human cerebrospinal fluid. Neurobiol Aging. 26, 207-227. 
[19]. Baird, G.S., et al. (2012). Age-dependent changes in the cerebrospinal fluid proteome by slow off-rate modified aptamer array. Am J Pathol. 180(2): 446-456.

[20]. Cooper, C.E., et al. (2013). Haptoglobin Binding Stabilizes Hemoglobin Ferryl Iron and the Globin Radical on Tyrosine $\beta 145$. Antioxid Redox Signal. 18(17), 22642273.

[21]. Wang, Y., Kinzie, E., Berger, F.G., Lim, S.K., Baumann, H. (2001).

Haptoglobin, an inflammation-inducible plasma protein. Redox Rep. 6(6):379-85.

[22]. Kantor, E., et al. (2014). Haptoglobin genotype and functional outcome after aneurysmal subarachnoid haemorrhage. J Neurosurg. 120(2), 386-390.

[23]. Chang, K.H., et al. (2007). Elevated haptoglobin level of cerebrospinal fluid in Guillain-Barré syndrome revealed by proteomics analysis. Proteomics - Clinical Applications. 1, 467-475.

[24]. Chang, K.H., et al. (2013). Analyses of haptoglobin level in the cerebrospinal fluid and serum of patients with neuromyelitis optica and multiple sclerosis. Clinica Chimica Acta. 417, 26-30.

[25]. Chamoun, V., et al. (2001). Haptoglobins as markers of blood-CSF barrier dysfunction: the findings in normal CSF. J Neurol Sci. 182 (2), 117-121.

[26]. Mbikay, M., Seidah, N., Chrétien, M. (2001). Neuroendocrine secretory protein 7B2: structure, expression and functions. Biochem. J. 357(2), 329-342.

[27]. Helwig, M., et al. (2012). The Neuroendocrine Protein 7B2 suppresses the aggregation of neurodegenerative disease-related proteins. J Biol. Chem. 288(2), 1114-1124. 
[28]. Suzuki, H., et al. (1989). Pituitary protein 7B2-like immunoreactivity in cerebrospinal fluid: comparison with other neuropeptides. J. Lab Clin. Med. 113(6), 743-748.

[29]. Wolf, S.A., Boddeke, H.W.G.M., Kettenmann H. (2017). Microglia in Physiology and Disease. Annu Rev Physiol. 79, 419-643.

[30]. Louveau, A., et al. (2015). Structural and functional features of central nervous system lymphatic vessels. Nature. 523, 337-341.

[31]. Boes, M. (2000). Role of natural and immune IgM antibodies in immune responses. Mol. Immun. 37(18), 1141-1149.

[32]. Tayebi, M., Collinge, J., Hawke, S. (2009). Unswitched immunoglobulin M response prolongs mouse survival in prion disease. J General Virology. 90, 777-782.

[33]. Svatonová, J. (2006). Critical evaluation of the biological role of $\operatorname{IgM}$ in cerebrospinal fluid in inflammatory and other diseases of the nervous system. Folia Microbiol (Praha). 51(5), 485-491.

[34]. Garg, A., Balthasar, J.P. (2009) Investigation of the Influence of FcRn on the Distribution of IgG to the Brain. AAPS J. 11(3), 553-557.

[35]. Biagioli, M., et al. (2009). Unexpected expression of alpha- and beta-globin in mesencephalic dopaminergic neurons and glial cells. Proc Natl Acad Sci U S A. $106(36), 15454-15459$.

[36]. Richter, F., Meurers, B.H., Zhu, C., Medvedeva, V.P., Chesselet, M.F. (2009). Neurons express hemoglobin alpha- and beta-chains in rat and human brains. J Comp Neurol. 515(5), 538-547.

[37]. Mast, H., Pöche, H., Marx, P. (1987). Hemoglobin quantitation in subarachnoid hemorrhage. Klin Wochenschr. 65(11), 513-515. 
[38]. Brown, N., et al. (2016). Neuronal hemoglobin expression and its relevance to multiple sclerosis neuropathology. J Mol Neurosci. 59, 1. doi:10.1007/s12031-0150711-6.

[39]. Henjum, K., et al. (2016). Cerebrospinal fluid soluble TREM2 in aging and Alzheimer's disease. Alzheimers Res Ther. 8(1), 17. doi: 10.1186/s13195-016-0182-1.

[40]. Franceschi, C., et al. (2007). Inflammaging and anti-inflammaging: a systemic perspective on aging and longevity emerged from studies in humans. Mech Ageing Dev. 128(1), 92-105.

[41]. Müller, L., Pawelec, G. (2014). Aging and immunity - Impact of behavioral intervention. Brain Behav.Immun. 39, 8-22.

[42]. Heneka, M.T., Kummer, M.P., Latz, E. (2014). Innate immune activation in neurodegenerative disease. Nat Rev Immunol. 14(7), 463-77.

[43]. Reid, S.J., Mckean, N.E., Henty, K., Portelius, E., Blennow,, K., Rudiger, S.R., et al. (2017) Alzheimer's disease markers in the aged sheep (Ovis aries). Neurobiol Aging. 58,112-19. 
Table 1. Functional classification of ovine CSF proteins

\begin{tabular}{lll} 
Protein function group & Protein number & Percentage (\%) \\
\hline Binding proteins & 31 & 21.7 \\
Peptide hormones & 14 & 9.8 \\
Enzymes for metabolism/signalling & 36 & 25.2 \\
Structural molecules & 23 & 16.1 \\
Immunoproteins & 19 & 13.3 \\
Coagulation proteins & 9 & 6.3 \\
Unknown & 11 & 7.7 \\
\hline Total & 143 & 100 \\
\hline
\end{tabular}


Table 2: A list of proteins showing more than $25 \%$ age-related increase in ovine CSF

\begin{tabular}{|c|c|c|c|c|c|c|}
\hline Protein name & $\begin{array}{l}\text { Accession } \\
\text { number }\end{array}$ & $\begin{array}{l}\text { Peptide } \\
\text { count }\end{array}$ & $\begin{array}{l}\text { Average } \\
\text { iTRAQ } \\
\text { ratio } \\
\text { young/IS }\end{array}$ & $\begin{array}{l}\text { Average } \\
\text { iTRAQ } \\
\text { ratio } \\
\text { middle- } \\
\text { age/IS }\end{array}$ & $\begin{array}{l}\text { Average } \\
\text { iTRAQ } \\
\text { ratio } \\
\text { old/IS }\end{array}$ & $\begin{array}{l}\text { \% } \\
\text { increase }\end{array}$ \\
\hline \multirow[t]{2}{*}{ Haptoglobin } & gi|258499 & 3 & $0.57[3]$ & $0.51[3]$ & $1.56[3]$ & \multirow{8}{*}{$\begin{array}{l}\text { Age- } \\
\text { related } \\
\text { increase } \\
>1 \text { fold }\end{array}$} \\
\hline & gi|998960 & 1 & $0.43[1]$ & $0.39[1]$ & $1.44[1]$ & \\
\hline \multirow[t]{2}{*}{ Haemoglobin } & $\begin{array}{l}\text { gi||122686 } \\
\text { beta subunit }\end{array}$ & 3 & $0.49[3]$ & $1.06[3]$ & $1.90[3]$ & \\
\hline & $\begin{array}{l}\text { gi|1787 } \\
\text { alpha subunit }\end{array}$ & 1 & $0.95[1]$ & $1.56[1]$ & $1.88[1]$ & \\
\hline Vimentin & gi|21757045 & 2 & $0.47[2]$ & $0.44[2]$ & $1.06[2]$ & \\
\hline IgM (Ig mu chain) & gi|165945 & 2 & $0.56[2]$ & $0.61[2]$ & $1.21[2]$ & \\
\hline $\begin{array}{l}\text { Neuroendrocrine } \\
\text { protein 7B2 isoform } 1\end{array}$ & gi|221139785 & 1 & $1.17[1]$ & $1.83[1]$ & $2.50[1]$ & \\
\hline $\begin{array}{l}\text { Fibrous sheath } \\
\text { interacting protein } 1\end{array}$ & gil28175039 & 1 & $0.23[1]$ & $0.34[1]$ & $0.78[1]$ & \\
\hline Transthyretin & gil57526651 & 7 & $0.63[11]$ & $\begin{array}{l}0.73 \\
{[11]}\end{array}$ & $\begin{array}{l}1.09 \\
{[11]}\end{array}$ & \multirow{14}{*}{$\begin{array}{l}\text { Age- } \\
\text { related } \\
\text { increase } \\
>50 \% \\
\text { but }<1 \\
\text { fold }\end{array}$} \\
\hline Serpin A3-1 & gil31340900 & 4 & $0.73[5]$ & $0.63[5]$ & $1.28[5]$ & \\
\hline Apolipoprotein C-III & gil47564119 & 3 & $0.74[3]$ & $1.09[3]$ & $1.22[3]$ & \\
\hline \multirow{2}{*}{$\begin{array}{l}\text { Inter-alpha-trypsin } \\
\text { inhibitor }\end{array}$} & gi159857769 & 2 & $0.95[2]$ & $1.16[2]$ & $1.57[2]$ & \\
\hline & gil48374067 & 2 & $0.82[2]$ & $1.12[2]$ & $1.68[2]$ & \\
\hline Actin & gil929752 & 1 & $0.78[1]$ & $0.82[1]$ & $1.55[1]$ & \\
\hline $\begin{array}{l}\text { Calcium uniporter } \\
\text { channel }\end{array}$ & gil833995 & 1 & $1.28[1]$ & $0.88[1]$ & $2.36[1]$ & \\
\hline $\begin{array}{l}\text { Thyroxine-binding } \\
\text { globulin }\end{array}$ & gil155369640 & 1 & $0.66[1]$ & $0.62[1]$ & $1.07[1]$ & \\
\hline $\begin{array}{l}\text { Beta-actin-like protein } \\
\text { 2-like }\end{array}$ & gil57043600 & 1 & $0.78[1]$ & $0.82[1]$ & $1.55[1]$ & \\
\hline $\begin{array}{l}\text { Retinoic acid receptor } \\
\text { responder protein } 2\end{array}$ & gil346716116 & 1 & $0.58[1]$ & $0.97[1]$ & $0.91[1]$ & \\
\hline Neuropeptide Y & gil30923117 & 1 & $0.78[1]$ & $1.4[1]$ & $1.54[1]$ & \\
\hline $\begin{array}{l}\text { Pyruvate kinase } \\
\text { isozymes } \mathrm{m} 1 / \mathrm{m} 2 \\
\text { isoform }\end{array}$ & gil33286418 & 1 & $1.05[1]$ & $0.59[1]$ & $1.61[1]$ & \\
\hline $\begin{array}{l}\text { Serum } \\
\text { paroxonase/arylesterase } \\
1\end{array}$ & gil126722853 & 1 & $0.99[1]$ & $0.91[1]$ & $2.18[1]$ & \\
\hline Complement C1r-B & gil164518925 & 1 & $0.91[1]$ & $0.91[1]$ & $1.44[1]$ & \\
\hline Ceruloplasmin & gil57617174 & 14 & $0.85[17]$ & $\begin{array}{l}0.84 \\
{[17]}\end{array}$ & $\begin{array}{l}1.15 \\
{[17]}\end{array}$ & \multirow{10}{*}{$\begin{array}{l}\text { Age- } \\
\text { related } \\
\text { increase } \\
>25 \% \\
\text { but } \\
<50 \%\end{array}$} \\
\hline Clusterin & gil27806907 & 7 & $0.81[9]$ & 0.93 [9] & $1.10[9]$ & \\
\hline IGF-2 & gil57164331 & 2 & $0.83[2]$ & $1.43[2]$ & $1.20[2]$ & \\
\hline IGFBP-2 & gil57164237 & 7 & $0.90[7]$ & $1.14[7]$ & $1.25[7]$ & \\
\hline IGFBP-4 & gil27807009 & 1 & $0.59[1]$ & $0.82[1]$ & $0.76[1]$ & \\
\hline IGFBP-5 & gil263306 & 1 & $1.69[1]$ & $1.32[1]$ & $2.19[1]$ & \\
\hline \multirow[t]{2}{*}{ IGFBP-6 } & gil56849568 & 1 & $0.39[1]$ & $0.72[1]$ & $0.82[1]$ & \\
\hline & gil5705897 & 1 & $0.79[1]$ & $1.13[1]$ & $0.96[1]$ & \\
\hline IGFBP-7 & gil4504619 & 3 & $0.75[3]$ & $0.61[3]$ & $1.01[3]$ & \\
\hline Beta 2-microglubin & gil57164311 & 4 & $0.91[4]$ & $0.99[4]$ & $1.17[4]$ & \\
\hline
\end{tabular}




\begin{tabular}{|c|c|c|c|c|c|}
\hline Primary amine oxidase & gil30794300 & 3 & $0.75[3]$ & $0.91[3]$ & $1.06[3]$ \\
\hline \multirow{4}{*}{ Complement $\mathrm{C} 4$} & gil1227 & 7 & $0.84[7]$ & $0.96[7]$ & $1.06[7]$ \\
\hline & gil1235 & 5 & $0.76[5]$ & $0.91[5]$ & $1.06[5]$ \\
\hline & gil50567362 & 4 & $0.87[4]$ & $1.08[4]$ & $1.14[4]$ \\
\hline & gil11314244 & 1 & $0.96[1]$ & $1.52[1]$ & $1.64[1]$ \\
\hline Complement C3 & gil4093220 & 1 & $0.76[1]$ & $0.96[1]$ & $1.03[1]$ \\
\hline Complement C6 & gil47115536 & 1 & $0.76[1]$ & $1.20[1]$ & $0.98[1]$ \\
\hline Complement C8 & gil841248 & 1 & $0.61[1]$ & $0.73[1]$ & $0.78[1]$ \\
\hline Chromogranin B & gil12644006 & 2 & $1.00[2]$ & $1.28[2]$ & $1.32[2]$ \\
\hline Alpha-macroglubin & gil290543549 & 2 & $0.78[2]$ & $0.92[2]$ & $1.17[2]$ \\
\hline \multirow[t]{2}{*}{ Beta-2 glycoprotein 1} & gil54792721 & 2 & $0.79[3]$ & $1.01[3]$ & 1.06 [3] \\
\hline & gil57528174 & 2 & $0.65[2]$ & $0.81[2]$ & $1.14[2]$ \\
\hline \multirow[t]{2}{*}{ Plasminogen } & gil833995 & 2 & $0.89[2]$ & $0.87[2]$ & $1.12[2]$ \\
\hline & gil51702791 & 1 & $0.83[1]$ & $0.99[1]$ & $1.12[1]$ \\
\hline $\begin{array}{l}\text { Proactivator } \\
\text { polypeptide }\end{array}$ & gil27806447 & 2 & $0.60[2]$ & $0.76[2]$ & $0.83[2]$ \\
\hline Apolipoprotein D & gil348582766 & 1 & $0.71[1]$ & $0.99[1]$ & $0.90[1]$ \\
\hline $\begin{array}{l}\text { Brain-type } \\
\text { ribonuclease }\end{array}$ & gil2381819 & 1 & $0.82[1]$ & $1.37[1]$ & $1.22[1]$ \\
\hline $\begin{array}{l}\text { Extracellular } \\
\text { superoxide dismutase }\end{array}$ & gil130508829 & 1 & $0.54[1]$ & $0.85[1]$ & $0.72[1]$ \\
\hline $\begin{array}{l}\text { Phosphatidylcholine } \\
\text { sterol acyltransferase }\end{array}$ & gil21542351 & 1 & $1.10[1]$ & $0.90[1]$ & $1.62[1]$ \\
\hline Eukephalin & gil223387 & 1 & $0.86[1]$ & $1.07[1]$ & $1.10[1]$ \\
\hline Neuroserpin & gil18158628 & 1 & $0.43[1]$ & $0.66[1]$ & $0.58[1]$ \\
\hline Alpha-2 antiplasmin & gil27807209 & 1 & $0.58[1]$ & $0.72[1]$ & $0.82[1]$ \\
\hline $\begin{array}{l}\text { Niemann-pick C2 } \\
\text { protein }\end{array}$ & gil28373999 & 1 & $0.74[1]$ & $0.71[1]$ & $0.94[1]$ \\
\hline Rheumatoid factor $\mathrm{Fab}$ & gil3659940 & 1 & $1.07[1]$ & $1.42[1]$ & $1.60[1]$ \\
\hline $\begin{array}{l}\text { Neuroblastoma } \\
\text { suppressor }\end{array}$ & gil13928832 & 1 & $1.04[1]$ & $1.07[1]$ & $1.35[1]$ \\
\hline Malate dehydrogenase & gi15174539 & 1 & $0.75[1]$ & $0.72[1]$ & $0.99[1]$ \\
\hline Alpha-1 antiproteinase & gil461443 & 1 & $0.28[1]$ & $0.28[1]$ & $0.40[1]$ \\
\hline Kininogen-1 & gil57109938 & 1 & $0.74[1]$ & $0.97[1]$ & $1.11[1]$ \\
\hline Fibromodalin & gil453157 & 1 & $0.78[1]$ & $0.84[1]$ & $0.91[1]$ \\
\hline
\end{tabular}

Column headings refer to the following: accession number is the reference for each protein in the NCBInr database; peptide count refers to the number of unique peptides with MS/MS ion scores used for protein identification; the average iTRAQ ratios are shown after data normalisation followed by the number of peptides used for quantification in square brackets. IS = internal standard. 
Table 3: A list of proteins showing more than $25 \%$ age-related decrease in ovine CSF

\begin{tabular}{|c|c|c|c|c|c|c|}
\hline Protein name & $\begin{array}{l}\text { Accession } \\
\text { number }\end{array}$ & $\begin{array}{l}\text { Peptide } \\
\text { count }\end{array}$ & $\begin{array}{l}\text { Average } \\
\text { iTRAQ } \\
\text { ratio } \\
\text { young/IS }\end{array}$ & $\begin{array}{l}\text { Average } \\
\text { iTRAQ } \\
\text { ratio } \\
\text { middle- } \\
\text { age/IS }\end{array}$ & $\begin{array}{l}\text { Average } \\
\text { iTRAQ } \\
\text { ratio } \\
\text { old/IS }\end{array}$ & $\begin{array}{l}\text { \% } \\
\text { decrease }\end{array}$ \\
\hline $\begin{array}{l}\text { Histon } \\
\text { deacetylase } 7\end{array}$ & gi|30913010 & 1 & $3.85[1]$ & $1.39[1]$ & $0.76[1]$ & $\begin{array}{l}\text { Age- } \\
\text { related } \\
\text { decrease } \\
>80 \%\end{array}$ \\
\hline \multirow[t]{3}{*}{ Fibrinogen } & gil1346006 & 3 & $1.25[3]$ & $1.40[3]$ & $0.74[3]$ & \multirow{20}{*}{$\begin{array}{l}\text { Age- } \\
\text { related } \\
\text { decrease } \\
>25 \% \text { but } \\
<50 \%\end{array}$} \\
\hline & gil3789962 & 3 & $1.03[3]$ & $1.15[3]$ & 0.87 [3] & \\
\hline & gil6980816 & 2 & $1.23[2]$ & $1.42[2]$ & $0.92[2]$ & \\
\hline $\begin{array}{l}\text { Neurosecretory } \\
\text { protein VGF }\end{array}$ & gill17136078 & 2 & $1.39[2]$ & $1.04[2]$ & $0.99[2]$ & \\
\hline $\begin{array}{l}\text { Beta-1,3-N- } \\
\text { actyl } \\
\text { glucosamingl } \\
\text { transferase } 6\end{array}$ & gil61553937 & 2 & $1.64[2]$ & $1.38[2]$ & $1.17[2]$ & \\
\hline Tetranectin & gil37409 & 2 & $1.23[2]$ & $1.37[2]$ & $0.83[2]$ & \\
\hline Transferrin & gil2318026 & 2 & $1.40[2]$ & $1.09[2]$ & $0.98[2]$ & \\
\hline \multirow[t]{2}{*}{ Calreticulin } & gil545920 & 2 & $0.95[2]$ & $1.11[2]$ & 0.64 [2] & \\
\hline & gil237420 & 1 & $0.91[1]$ & $0.99[1]$ & $0.55[1]$ & \\
\hline Contactin-2 & gil4827022 & 2 & $1.25[2]$ & $0.73[2]$ & $0.77[2]$ & \\
\hline $\begin{array}{l}\mathrm{V} \text {-set and } \\
\text { transmembrane } \\
\text { domain protein } \\
2 \mathrm{~A}\end{array}$ & gil20306326 & 1 & $1.12[2]$ & $0.81[2]$ & $0.59[2]$ & \\
\hline Cadherin-15 & gil4826669 & 1 & $1.39[1]$ & $0.89[1]$ & $0.72[1]$ & \\
\hline $\begin{array}{l}\text { Chain A, } \\
\text { Divalent cation } \\
\text { tolerant protein } \\
\text { cuta }\end{array}$ & gil56554578 & 1 & $1.37[1]$ & $1.04[1]$ & $1.00[1]$ & \\
\hline $\begin{array}{l}\text { Cell adhesion } \\
\text { molecule } 4\end{array}$ & gil21686977 & 1 & $1.11[1]$ & $0.50[1]$ & $0.77[1]$ & \\
\hline $\begin{array}{l}\text { Adipsin / } \\
\text { complement } \\
\text { factor D }\end{array}$ & gil773265 & 1 & $1.50[1]$ & $1.27[1]$ & $1.09[1]$ & \\
\hline Nidogen-2 & gil2791962 & 1 & $1.35[1]$ & $1.22[1]$ & $0.70[1]$ & \\
\hline Protein $\mathrm{C}$ & gil163487 & 1 & $0.93[1]$ & $0.68[1]$ & $0.69[1]$ & \\
\hline $\begin{array}{l}\text { Neurocan core } \\
\text { protein }\end{array}$ & gil77681353 & 1 & $1.11[1]$ & $0.71[1]$ & $0.61[1]$ & \\
\hline $\begin{array}{l}\text { Protein kinase } \\
\text { C-binding } \\
\text { protein NELL-2 }\end{array}$ & gil2494290 & 1 & $1.81[1]$ & $1.40[1]$ & $1.00[1]$ & \\
\hline $\begin{array}{l}\text { Nuclear factor } \\
\text { of activated T } \\
\text { cells } \\
\text { cytoplasmic } \\
\text { isoform } 2\end{array}$ & gil27886541 & 1 & $0.96[1]$ & $1.56[1]$ & $0.38[1]$ & \\
\hline
\end{tabular}

Column headings refer to the following: accession number is the reference for each protein in the NCBInr database; peptide count refers to the number of unique peptides 
with MS/MS ion scores used for protein identification; the average iTRAQ ratios are shown after data normalisation followed by the number of peptides used for quantification in square brackets. IS = internal standard. 
A

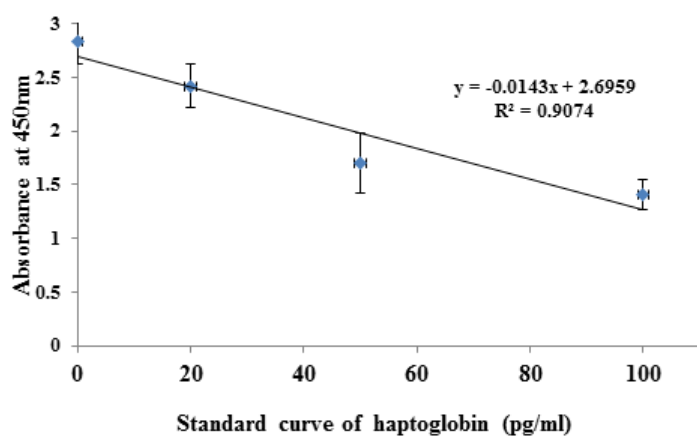

C

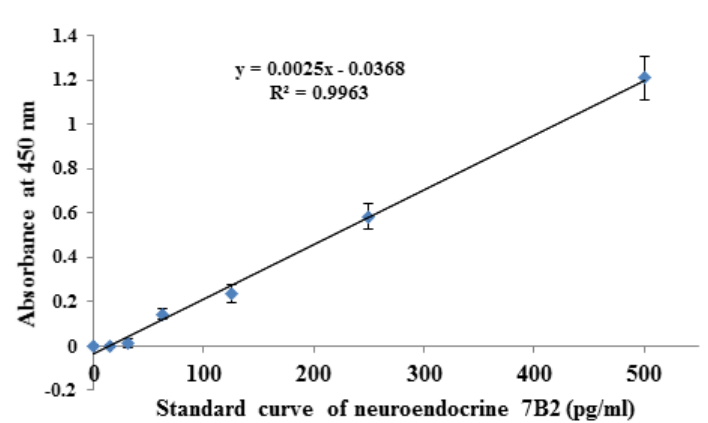

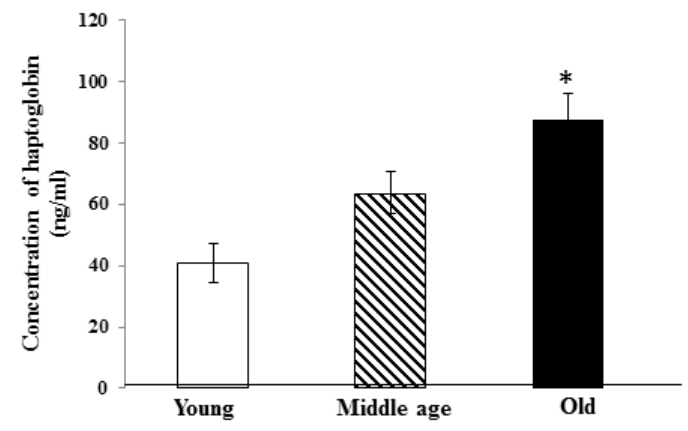

D

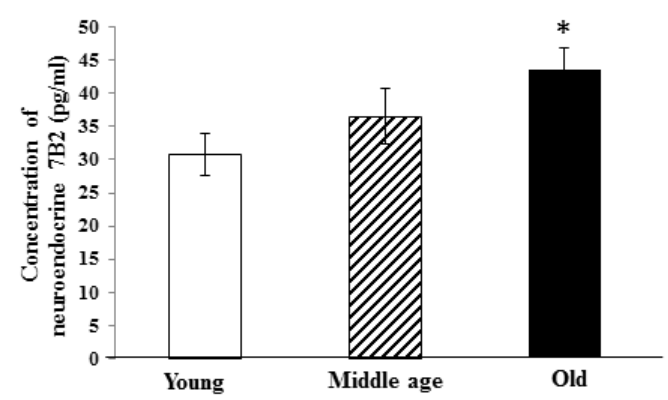

Figure 1. Concentration of haptoglobin (HP) and neuroendocrine protein 7B2 in CSF of young, middle age, and old groups by ELISA assay. A, C are standard curves of $\mathrm{Hp}(\mathrm{A})$ and neuroendocrine 7B2 (C); B and D summarized the concentrations of Hp (B) and neuroendocrine 7B2 (D) in CSF from different age groups, $\mathrm{n}=7 .{ }^{*}$ compared to young group, $\mathrm{P}<0.01$. 
A

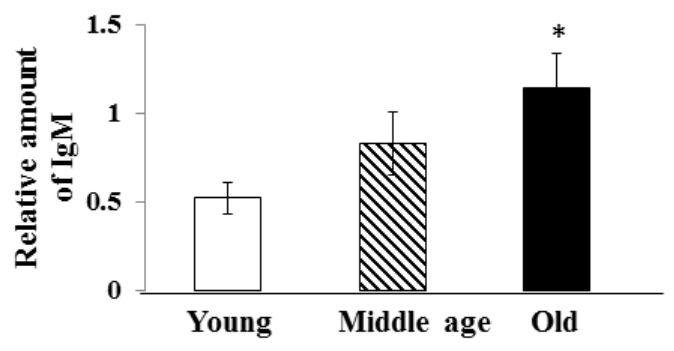

B

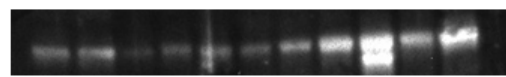

$\begin{array}{lllllllllll}1 & 2 & 3 & 4 & 5 & 6 & 7 & 8 & 9 & 10 & 11\end{array}$

C

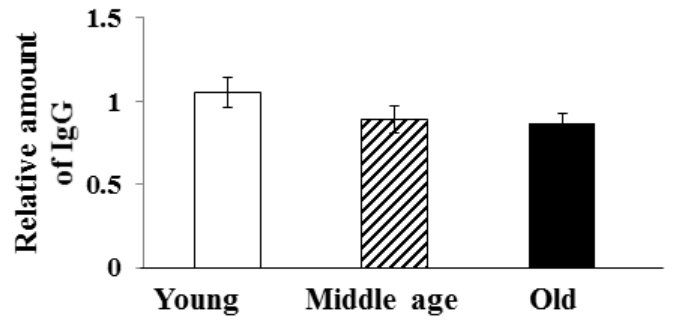

D

$\begin{array}{llllllllllll}1 & 2 & 3 & 4 & 5 & 6 & 7 & 8 & 9 & 10 & 11\end{array}$

Figure 2. Relative amount of $\operatorname{IgM}(\mathrm{A})$ and $\operatorname{IgG}(\mathrm{C})$ in $\mathrm{CSF}$ between different ages, which was based on their band density of Western immunoblotting image of $\operatorname{IgM}(\mathrm{B})$ and $\operatorname{IgG}(\mathrm{D})$ respectively. In image $\mathrm{B}$ or D, lanes 1-4: young CSF; lanes 5-7: middle aged CSF; lanes 8-10: old CSF; lane 11: molecular size marker. *compared to young group, $\mathrm{P}<0.01$ 


\begin{tabular}{|c|c|c|c|c|c|c|c|c|c|c|c|c|c|}
\hline \multirow[b]{2}{*}{ Protein common name } & \multicolumn{2}{|l|}{ Protein } & \multirow{2}{*}{$\begin{array}{l}\text { Peptide } \\
\text { count }\end{array}$} & \multirow{2}{*}{$\begin{array}{l}\text { Total } \\
\text { ion } \\
\text { score }\end{array}$} & \multicolumn{3}{|c|}{ Average iTRAQ ratio } & \multicolumn{3}{|c|}{ iTRAQ Standard Deviation } & \multicolumn{3}{|c|}{ iTRAQ Peptides } \\
\hline & MW & PI & & & 114/117 & $115 / 117$ & 116/117 & 114/117 & $115 / 117$ & $116 / 117$ & 114/117 & $115 / 117$ & $116 / 117$ \\
\hline $\begin{array}{l}\text { Serum albumin precursor } \\
\text { Serotransferrin; } \\
\text { Transferrin; }\end{array}$ & 79687 & 6 & 43 & 2879 & 1 & 2 & 1.1 & 0.6 & 0.7 & 0.3 & 147 & 147 & 147 \\
\hline Beta-1 metal-binding globulin; & 88817 & 7 & 20 & 1321 & 1 & 1 & 1.1 & 0.5 & 0.6 & 0.4 & 36 & 36 & 36 \\
\hline Serum albumin; & 79648 & 6 & 18 & 1217 & 1 & 2 & 1.1 & 0.7 & 0.7 & 0.4 & 70 & 70 & 70 \\
\hline Angiotensinogen; & 54004 & 7 & 15 & 1182 & 1 & 1 & 1.1 & 0.6 & 0.6 & 0.3 & 19 & 19 & 19 \\
\hline Apolipoprotein A-I, apoA-1 & 31729 & 6 & 15 & 1043 & 1 & 1 & 1.2 & 0.5 & 0.4 & 0.3 & 27 & 27 & 27 \\
\hline Ceruloplasmin precursor & 129919 & 6 & 14 & 1038 & 1 & 1 & 1.4 & 0.4 & 1.0 & 0.5 & 17 & 17 & 17 \\
\hline Complement C3 & 205367 & 6 & 15 & 969 & 1 & 1 & 1.1 & 0.4 & 0.4 & 0.2 & 23 & 23 & 23 \\
\hline Alpha-1-antiproteinase precursor & 50699 & 6 & 13 & 804 & 1 & 1 & 1.3 & 0.6 & 0.5 & 0.4 & 19 & 19 & 19 \\
\hline Apolipoprotein E; & 38112 & 6 & 10 & 687 & 1 & 1 & 1.3 & 0.4 & 0.3 & 0.2 & 16 & 16 & 16 \\
\hline Complement component & 43395 & 6 & 10 & 576 & 1 & 1 & 1.2 & 0.6 & 0.4 & 0.3 & 10 & 10 & 10 \\
\hline Preprocomplement component C3 & 205042 & 6 & 9 & 570 & 1 & 1 & 1.1 & 0.3 & 0.3 & 0.2 & 16 & 16 & 16 \\
\hline Transferrin & 86771 & 7 & 7 & 549 & 1 & 1 & 1.0 & 0.4 & 0.4 & 0.3 & 16 & 16 & 16 \\
\hline $\begin{array}{l}\text { Alpha-2-HS-glycoprotein precursor } \\
\text { Immunoglobulin lambda light }\end{array}$ & 41651 & 5 & 6 & 534 & 1 & 1 & 1.0 & 0.4 & 0.6 & 0.3 & 8 & 8 & 8 \\
\hline chain constant region segment 1 & 13045 & 8 & 5 & 525 & 1 & 1 & 1.3 & 0.5 & 0.6 & 0.3 & 15 & 15 & 15 \\
\hline Keratin 1 & 70200 & 8 & 8 & 504 & 3 & 5 & 1.8 & 1.1 & 1.6 & 0.6 & 10 & 10 & 10 \\
\hline $\begin{array}{l}\text { Alpha-1-antiproteinase precursor } \\
\text { Ig lambda chain C region - sheep }\end{array}$ & 51106 & 6 & 9 & 502 & 1 & 1 & 1.3 & 0.6 & 0.6 & 0.4 & 13 & 13 & 13 \\
\hline (fragment) & 13172 & 8 & 4 & 495 & 1 & 1 & 1.3 & 0.5 & 0.5 & 0.2 & 15 & 15 & 15 \\
\hline Complement $\mathrm{C} 3$ preproprotein & 204039 & 6 & 8 & 483 & 1 & 1 & 1.1 & 0.3 & 0.4 & 0.2 & 14 & 14 & 14 \\
\hline Contactin-1 precursor & 122263 & 6 & 6 & 481 & 2 & 2 & 1.4 & 1.0 & 1.2 & 0.5 & 7 & 7 & 7 \\
\hline Transthyretin precursor & 17582 & 6 & 7 & 464 & 1 & 1 & 1.3 & 0.4 & 0.7 & 0.4 & 11 & 11 & 11 \\
\hline Complement component $\mathrm{C} 4$ & 15520 & 5 & 7 & 463 & 1 & 1 & 1.3 & 0.4 & 0.5 & 0.3 & 7 & 7 & 7 \\
\hline Complement $\mathrm{C} 4$ & 108118 & 6 & 9 & 458 & 1 & 2 & 1.3 & 0.5 & 0.5 & 0.4 & 9 & 9 & 9 \\
\hline Clusterin preproprotein & 55864 & 6 & 7 & 455 & 1 & 1 & 1.3 & 0.4 & 0.4 & 0.2 & 9 & 9 & 9 \\
\hline Albumin & 79319 & 6 & 7 & 442 & 1 & 2 & 1.1 & 1.3 & 1.8 & 0.7 & 21 & 21 & 21 \\
\hline Fibronectin & 95323 & 9 & 6 & 421 & 2 & 1 & 1.2 & 0.9 & 1.1 & 0.4 & 7 & 7 & 7 \\
\hline Keratin, type I cytoskeletal 9 & 66102 & 5 & 4 & 411 & 2 & 4 & 1.7 & 1.6 & 3.5 & 0.8 & 4 & 4 & 4 \\
\hline
\end{tabular}


Immunoglobulin gamma-1 chain Antithrombin-III precursor

Insulin-like growth factor-binding protein 2 precursor

Pigment epithelium-derived factor precursor

Gelsolin

Actin-depolymerizing factor;

Prostaglandin-H2 D-isomerase

Beta-2-microglobulin precursor

Plasminogen

Ectonucleotide

pyrophosphatase/phosphodiesterase

family member 2

Complement component $\mathrm{C} 4$

Chain A, crystal structure of the

first active autolysate form of the

porcine alpha trypsin

Chain E, leech-derived tryptase

inhibitortrypsin complex

Serpin A3-1 precursor

Amyloid beta A4 protein isoform $b$ precurso

72 kda type IV collagenase

precurso

Phospholipid transfer protein

Insulin-like growth factor-binding

protein 7

Keratin, type II cytoskeletal 2

epidermal

Brevican core protein

Fibrinogen beta chain;

Fibrinopeptide B

Apolipoprotein A-I

Chain A, crystal structure of bovine

holo-rbp at ph 4.0

Complement component 4 , gene 2

\begin{tabular}{|c|c|c|c|c|c|c|c|c|c|c|c|}
\hline 55492 & 6 & 6 & 399 & 1 & 1 & 1.1 & 0.5 & 0.4 & 0.2 & 14 & 14 \\
\hline 57635 & 6 & 7 & 397 & 1 & 2 & 1.2 & 0.3 & 0.2 & 0.2 & 7 & 7 \\
\hline 37202 & 7 & 7 & 394 & 1 & 2 & 1.5 & 0.4 & 0.7 & 0.3 & 7 & 7 \\
\hline 50039 & 7 & 5 & 388 & 2 & 2 & 1.6 & 1.1 & 1.7 & 0.3 & 5 & 5 \\
\hline 91483 & 6 & 6 & 371 & 1 & 2 & 1.2 & 0.4 & 0.5 & 0.3 & 6 & 6 \\
\hline 22795 & 6 & 4 & 343 & 1 & 2 & 1.2 & 0.5 & 0.5 & 0.3 & 8 & 8 \\
\hline 14845 & 6 & 4 & 340 & 1 & 1 & 1.4 & 0.4 & 0.6 & 0.2 & 4 & 4 \\
\hline 41059 & 8 & 4 & 322 & 1 & 2 & 1.4 & 0.3 & 0.5 & 0.2 & 4 & 4 \\
\hline 111363 & 7 & 7 & 316 & 1 & 2 & 1.4 & 0.6 & 0.8 & 0.4 & 7 & 7 \\
\hline 15731 & 6 & 5 & 287 & 1 & 1 & 1.3 & 0.4 & 0.4 & 0.3 & 5 & 5 \\
\hline 14234 & 8 & 3 & 286 & 1 & 1 & 1.1 & 0.4 & 0.4 & 0.1 & 5 & 5 \\
\hline 25594 & 8 & 3 & 286 & 1 & 1 & 1.1 & 0.4 & 0.4 & 0.1 & 5 & 5 \\
\hline 49726 & 6 & 4 & 278 & 1 & 1 & 1.5 & 0.4 & 0.5 & 0.4 & 5 & 5 \\
\hline 91357 & 5 & 4 & 276 & 1 & 1 & 1.1 & 0.4 & 0.4 & 0.3 & 5 & 5 \\
\hline 81088 & 5 & 5 & 275 & 1 & 1 & 1.2 & 0.8 & 0.3 & 0.3 & 5 & 5 \\
\hline 59170 & 6 & 3 & 256 & 1 & 2 & 1.1 & 0.5 & 0.7 & 0.3 & 4 & 4 \\
\hline 32245 & 8 & 3 & 253 & 1 & 1 & 1.2 & 0.9 & 0.4 & 0.4 & 3 & 3 \\
\hline 70811 & 8 & 3 & 243 & 2 & 2 & 1.2 & 0.1 & 0.3 & 0.2 & 3 & 3 \\
\hline 103281 & 5 & 3 & 241 & 2 & 2 & 1.5 & 0.3 & 0.4 & 0.3 & 3 & 3 \\
\hline 59432 & 8 & 3 & 239 & 1 & 2 & 0.9 & 0.2 & 0.0 & 0.1 & 3 & 3 \\
\hline 33204 & 5 & 3 & 232 & 1 & 1 & 1.1 & 0.3 & 0.4 & 0.3 & 5 & 5 \\
\hline 22019 & 5 & 4 & 230 & 1 & 2 & 1.3 & 0.3 & 0.4 & 0.1 & 6 & 6 \\
\hline 205543 & 7 & 4 & 228 & 1 & 2 & 1.4 & 0.3 & 0.5 & 0.4 & 4 & 4 \\
\hline
\end{tabular}




\begin{tabular}{|c|c|c|c|c|c|c|c|c|c|c|c|c|}
\hline Hypothetical protein & 263095 & 6 & 3 & 227 & 1 & 1 & 1.0 & 0.4 & 0.3 & 0.2 & 3 & 3 \\
\hline Keratin 10 & 62434 & 5 & 3 & 221 & 2 & 2 & 1.2 & 0.8 & 0.6 & 0.3 & 3 & 3 \\
\hline $\begin{array}{l}\text { Hemoglobin subunit beta; Beta- } \\
\text { globin }\end{array}$ & 17983 & 7 & 3 & 219 & 1 & 2 & 2.3 & 0.1 & 0.2 & 0.2 & 3 & 3 \\
\hline $\begin{array}{l}\text { Inter-alpha-trypsin inhibitor heavy } \\
\text { chain } \mathrm{H} 2 \text { precursor }\end{array}$ & 114147 & 7 & 4 & 217 & 1 & 2 & 1.2 & 0.6 & 0.6 & 0.3 & 4 & 4 \\
\hline $\begin{array}{l}\text { Neurosecretory protein VGF } \\
\text { precursor }\end{array}$ & 69569 & 5 & 2 & 203 & 2 & 2 & 1.2 & 0.3 & 0.5 & 0.3 & 2 & 2 \\
\hline Fibronectin & 69908 & 8 & 2 & 199 & 2 & 1 & 1.3 & 0.2 & 0.5 & 0.0 & 2 & 2 \\
\hline Prothrombin & 76294 & 6 & 4 & 196 & 1 & 1 & 1.2 & 0.1 & 0.3 & 0.7 & 4 & 4 \\
\hline Pigment epithelium-derived factor & 50486 & 6 & 3 & 196 & 2 & 2 & 1.6 & 0.3 & 0.4 & 0.2 & 3 & 3 \\
\hline Factor xiia inhibitor precursor & 55333 & 6 & 3 & 193 & 1 & 2 & 1.5 & 0.3 & 0.2 & 0.3 & 3 & 3 \\
\hline Apolipoprotein C-III precursor & 11982 & 5 & 3 & 186 & 1 & 2 & 1.4 & 0.0 & 0.1 & 0.1 & 3 & 3 \\
\hline $\begin{array}{l}\text { Primary amine oxidase, lung } \\
\text { isozyme }\end{array}$ & 87835 & 6 & 3 & 184 & 1 & 1 & 1.3 & 0.1 & 0.1 & 0.2 & 3 & 3 \\
\hline Sex hormone-binding globulin & 41550 & 5 & 2 & 172 & 2 & 2 & 1.4 & 0.4 & 0.9 & 0.1 & 2 & 2 \\
\hline Apolipoprotein A-IV precursor & 48023 & 6 & 3 & 171 & 1 & 1 & 1.1 & 0.5 & 0.4 & 0.1 & 4 & 4 \\
\hline Complement $\mathrm{C} 3$ alpha chain & 90597 & 5 & 2 & 168 & 1 & 2 & 1.2 & 0.5 & 0.1 & 0.2 & 3 & 3 \\
\hline $\begin{array}{l}\text { Beta-1,3-N- } \\
\text { acetylglucosaminyltransferase }\end{array}$ & & & & & & & & & & & & \\
\hline bgnt-6 & 45814 & 6 & 2 & 164 & 2 & 2 & 1.4 & 0.4 & 0.4 & 0.1 & 2 & 2 \\
\hline Serum albumin & 78092 & 6 & 4 & 162 & 1 & 2 & 1.1 & 0.4 & 0.7 & 0.2 & 6 & 6 \\
\hline DKFZP459P137 protein & 58526 & 5 & 3 & 162 & 1 & 1 & 1.4 & 0.2 & 0.3 & 0.3 & 3 & 3 \\
\hline $\begin{array}{l}\text { Collagen alpha-1(XII) chain long } \\
\text { isoform precursor }\end{array}$ & 356530 & 5 & 2 & 159 & 1 & 1 & 1.1 & 0.0 & 0.1 & 0.2 & 2 & 2 \\
\hline Pigment epithelium-derived factor & 49797 & 6 & 2 & 157 & 2 & 2 & 1.5 & 0.1 & 0.3 & 0.2 & 2 & 2 \\
\hline Transcription factor AEBP1 & 85223 & 5 & 3 & 155 & 1 & 1 & 1.4 & 0.1 & 0.6 & 0.3 & 3 & 3 \\
\hline Fibrinogen A-alpha chain & 46124 & 6 & 3 & 155 & 1 & 2 & 1.0 & 0.2 & 0.1 & 0.2 & 3 & 3 \\
\hline $\begin{array}{l}\text { Haptoglobin heavy chain, hph } \\
\text { chain }\end{array}$ & 30384 & 6 & 3 & 153 & 1 & 1 & 1.9 & 0.2 & 0.2 & 0.3 & 3 & 3 \\
\hline Chromogranin B & 79125 & 5 & 2 & 151 & 1 & 2 & 1.6 & 0.1 & 0.1 & 0.0 & 2 & 2 \\
\hline Metalloproteinase inhibitor 2 & 25216 & 7 & 2 & 149 & 1 & 1 & 1.1 & 0.2 & 0.1 & 0.1 & 2 & 2 \\
\hline Chitinase-3-like protein 1 & 46169 & 9 & 3 & 148 & 1 & 1 & 1.5 & 0.2 & 0.3 & 0.3 & 3 & 3 \\
\hline Tetranectin & 25324 & 6 & 2 & 145 & 1 & 2 & 1.0 & 0.2 & 0.1 & 0.6 & 2 & 2 \\
\hline Vitamin D-binding protein & 28956 & 5 & 3 & 140 & 1 & 1 & 1.2 & 0.4 & 0.3 & 0.4 & 4 & 4 \\
\hline Transferrin & 7637 & 8 & 2 & 137 & 2 & 2 & 1.2 & 0.3 & 0.1 & 0.0 & 2 & 2 \\
\hline
\end{tabular}


Limbic system-associated membrane protein precursor

Fibrinogen alpha chain

Insulin-like growth factor II

preproprotein

Complement component $\mathrm{C} 7$

precursor

Unnamed protein product

Dickkopf-related protein 3

precursor

Beta-2-glycoprotein 1 precursor

WAP, kazal, immunoglobulin,

kunitz and NTR domain-containing protein 2 precursor

Inter-alpha (globulin) inhibitor $\mathrm{H} 4$

$\begin{array}{rlll}40309 & 6 & 2 & 136 \\ 1991 & 4 & 1 & 136 \\ 20980 & 9 & 2 & 134 \\ 103502 & 7 & 2 & 130 \\ 55266 & 5 & 2 & 130 \\ 40484 & 5 & 2 & 129 \\ 43614 & 9 & 2 & 129\end{array}$

68543

Calcium uniporter channel

component [cattle, heart, Peptide

Mitochondrial Partial, 18 aa,

segment 3

Plasminogen precursor

Calreticulin

Amyloid precursor-like protein 1

Unnamed protein product

Immunoglobulin kappa light chain

constant region

Insulin-like growth factor-binding protein 7

Beta-2-glycoprotein 1

Superoxide dismutase [Cu-Zn]

Immunoglobulin lambda-6c light

chain variable region

Alpha-macroglobulin

Igg1 heavy chain constant region

Myocilin; Trabecular meshwork-

induced glucocorticoid response

protein;

Immunoglobulin mu chain

$\begin{array}{lllllll}1.0 & 0.1 & 0.2 & 0.1 & 2 & 2 & 2 \\ 1.7 & 0.0 & 0.0 & 0.0 & 1 & 1 & 1 \\ 1.4 & 0.1 & 0.0 & 0.0 & 2 & 2 & 2 \\ 1.0 & 0.2 & 0.0 & 0.1 & 2 & 2 & 2 \\ 1.1 & 0.1 & 0.3 & 0.0 & 2 & 2 & 2 \\ 0.9 & 0.5 & 0.2 & 0.2 & 3 & 3 & 3 \\ 1.3 & 0.4 & 0.7 & 0.1 & 3 & 3 & 3 \\ & & & & & & \\ 1.1 & 0.2 & 0.1 & 0.0 & 2 & 2 & 2 \\ 1.9 & 0.0 & 0.1 & 0.2 & 2 & 2 & 2\end{array}$

\begin{tabular}{|c|c|c|c|c|c|c|c|c|c|c|c|}
\hline 2470 & 5 & 1 & 123 & 2 & 1 & 2.8 & 0.0 & 0.0 & 0.0 & 1 & 1 \\
\hline 101001 & 8 & 2 & 121 & 1 & 1 & 1.3 & 0.1 & 0.2 & 0.1 & 2 & 2 \\
\hline 52543 & 4 & 2 & 119 & 1 & 2 & 0.8 & 0.1 & 0.2 & 0.1 & 2 & 2 \\
\hline 74871 & 6 & 2 & 117 & 1 & 1 & 0.9 & 0.4 & 0.7 & 0.2 & 2 & 2 \\
\hline 55767 & 5 & 2 & 116 & 1 & 1 & 1.3 & 0.1 & 0.1 & 0.1 & 2 & 2 \\
\hline 13396 & 6 & 2 & 114 & 2 & 2 & 1.1 & 0.4 & 0.1 & 0.1 & 3 & 3 \\
\hline 32027 & 8 & 2 & 113 & 1 & 1 & 1.4 & 0.1 & 0.0 & 0.3 & 2 & 2 \\
\hline 43957 & 9 & 2 & 109 & 1 & 1 & 1.3 & 0.2 & 0.4 & 0.2 & 2 & 2 \\
\hline 17556 & 6 & 2 & 106 & 1 & 1 & 1.1 & 0.1 & 0.1 & 0.1 & 2 & 2 \\
\hline 12870 & 5 & 1 & 106 & 2 & 1 & 1.5 & 0.0 & 0.0 & 0.0 & 1 & 1 \\
\hline 178259 & 6 & 2 & 104 & 1 & 1 & 1.4 & 0.4 & 0.4 & 0.2 & 2 & 2 \\
\hline 39259 & 6 & 2 & 101 & 1 & 1 & 1.2 & 0.8 & 0.7 & 0.3 & 7 & 7 \\
\hline 58155 & 5 & 2 & 99 & 2 & 2 & 1.2 & 0.3 & 0.4 & 0.5 & 2 & 2 \\
\hline 57811 & 5 & 2 & 99 & 1 & 1 & 1.4 & 0.0 & 0.1 & 0.0 & 2 & 2 \\
\hline
\end{tabular}


Chain c, the crystal structure of modified bovine fibrinogen

Proactivator polypeptide

Apolipoprotein D, apod

Insulin-like growth factor-binding

protein 6

Hepatocarcinogenesis-specific

protein/hemopexin homolog

Inter-alpha-trypsin inhibitor heavy

chain $\mathrm{H} 4$

Phospholipid transfer protein

Inhibitor of carbonic anhydrase

precursor

Unnamed protein product

Collagen type I alpha 1

Contactin-2 precursor

Fibulin-1

Cadherin-15 preproprotein

Vitamin K-dependent protein S

precurso

Apolipoprotein D-like

Unnamed protein product

Glutaminyl cyclase

Chain a, divalent cation tolerant protein cuta

Vitronectin precursor

V-type proton atpase subunit S1

precursor

\begin{tabular}{|c|c|c|c|c|c|c|c|c|c|c|c|}
\hline 51472 & 5 & 2 & 98 & 1 & 2 & 1.1 & 0.1 & 0.1 & 0.0 & 2 & 2 \\
\hline 64672 & 5 & 2 & 97 & 1 & 1 & 1.0 & 0.1 & 0.4 & 0.1 & 2 & 2 \\
\hline 31277 & 5 & 1 & 95 & 2 & 2 & 1.4 & 0.0 & 0.0 & 0.0 & 1 & 1 \\
\hline 14741 & 11 & 1 & 93 & 1 & 2 & 1.1 & 0.0 & 0.0 & 0.0 & 1 & 1 \\
\hline 33129 & 9 & 2 & 93 & 2 & 2 & 1.6 & 0.4 & 0.4 & 0.3 & 3 & 3 \\
\hline 108849 & 6 & 2 & 90 & 1 & 2 & 2.0 & 0.1 & 0.2 & 0.0 & 2 & 2 \\
\hline 57667 & 7 & 2 & 89 & 1 & 1 & 1.0 & 0.2 & 0.4 & 0.2 & 2 & 2 \\
\hline 85724 & 6 & 2 & 89 & 1 & 1 & 1.1 & 0.0 & 0.2 & 0.3 & 2 & 2 \\
\hline 12782 & 5 & 2 & 89 & 1 & 1 & 1.4 & 0.0 & 0.2 & 0.3 & 2 & 2 \\
\hline 10249 & 5 & 1 & 87 & 2 & 3 & 1.3 & 0.0 & 0.0 & 0.0 & 1 & 1 \\
\hline 119638 & 8 & 2 & 87 & 2 & 1 & 0.9 & 0.1 & 0.1 & 0.0 & 2 & 2 \\
\hline 70865 & 5 & 2 & 85 & 1 & 2 & 1.2 & 0.7 & 0.3 & 0.4 & 2 & 2 \\
\hline 91489 & 5 & 1 & 84 & 2 & 1 & 0.9 & 0.0 & 0.0 & 0.0 & 1 & 1 \\
\hline 83178 & 5 & 2 & 83 & 1 & 1 & 0.7 & 0.4 & 0.2 & 0.1 & 2 & 2 \\
\hline 23365 & 5 & 1 & 83 & 1 & 1 & 1.1 & 0.0 & 0.0 & 0.0 & 1 & 1 \\
\hline 75687 & 6 & 2 & 81 & 2 & 2 & 1.3 & 0.5 & 0.2 & 0.2 & 2 & 2 \\
\hline 28410 & 7 & 1 & 80 & 3 & 2 & 2.4 & 0.0 & 0.0 & 0.0 & 1 & 1 \\
\hline 16967 & 6 & 1 & 79 & 2 & 2 & 1.2 & 0.0 & 0.0 & 0.0 & 1 & 1 \\
\hline 57941 & 6 & 1 & 79 & 1 & 1 & 1.4 & 0.0 & 0.0 & 0.0 & 1 & 1 \\
\hline 53615 & 5 & 1 & 77 & 1 & 1 & 0.9 & 0.0 & 0.0 & 0.0 & 1 & 1 \\
\hline 52957 & 4 & 1 & 76 & 1 & 1 & 0.6 & 0.0 & 0.0 & 0.0 & 1 & 1 \\
\hline 20326 & 10 & 1 & 75 & 1 & 2 & 1.4 & 0.0 & 0.0 & 0.0 & 1 & 1 \\
\hline 14422 & 9 & 1 & 73 & 1 & 1 & 1.3 & 0.0 & 0.0 & 0.0 & 1 & 1 \\
\hline 105492 & 6 & 1 & 73 & 1 & 1 & 1.3 & 0.0 & 0.0 & 0.0 & 1 & 1 \\
\hline 37821 & 5 & 1 & 73 & 1 & 1 & 1.3 & 0.0 & 0.0 & 0.0 & 1 & 1 \\
\hline 15391 & 10 & 1 & 71 & 1 & 1 & 1.1 & 0.0 & 0.0 & 0.0 & 1 & 1 \\
\hline 26551 & 6 & 1 & 69 & 1 & 1 & 1.4 & 0.0 & 0.0 & 0.0 & 1 & 1 \\
\hline
\end{tabular}

Brain-type ribonuclease

Similar to pro alpha $1(\mathrm{I})$ collagen

Alpha 1 (I) chain propeptide

Type I collagen alpha 1 chain

$\mathrm{C}$-type natriuretic peptide precursor

Immunoglobulin lambda light

chain

26551 
Extracellular superoxide dismutase

$[\mathrm{Cu}-\mathrm{Zn}]$ precursor

Thyroxine-binding globulin

precursor

Insulin-like growth factor-binding

protein 5

CCP modules 3-12, with parts of

CCP 2 and 13

Ig gamma heavy chain constant

region - rabbit (fragment)

Phosphatidylcholine-sterol

acyltransferase; Lecithin-

cholesterol acyltr

Plasminogen

Neuronal pentraxin-1 precursor

SPARC precursor

Type I collagen alpha 1 precursor

Enkephalin precursor

Hypothetical protein LOC478604 isoform 1

Cd14

Chain c, crystal structure of mouse

neuroserpin

Calsyntenin-1 isoform 1

Fibrinogen A-alpha-chain

Dystroglycan

Unnamed protein product

Beta-actin-like protein 2-like

Truncated tenascin XB

$\mathrm{V}$-set and transmembrane domain

containing 2A

Golph2

Immunoglobulin V lambda chain

Immunoglobulin lambda light

chain VLJ region

Immunoglobulin light chain

variable region

\begin{tabular}{|c|c|c|c|c|c|c|c|c|c|c|c|}
\hline 26858 & 7 & 1 & 68 & 1 & 1 & 0.9 & 0.0 & 0.0 & 0.0 & 1 & 1 \\
\hline 50564 & 6 & 1 & 67 & 1 & 1 & 1.3 & 0.0 & 0.0 & 0.0 & 1 & 1 \\
\hline 13885 & 6 & 1 & 66 & 2 & 2 & 2.6 & 0.0 & 0.0 & 0.0 & 1 & 1 \\
\hline 84142 & 6 & 1 & 66 & 2 & 2 & 1.5 & 0.0 & 0.0 & 0.0 & 1 & 1 \\
\hline 22719 & 8 & 1 & 66 & 0 & 1 & 1.1 & 0.0 & 0.0 & 0.2 & 2 & 2 \\
\hline 22082 & 5 & 1 & 66 & 1 & 1 & 1.9 & 0.0 & 0.0 & 0.0 & 1 & 1 \\
\hline 15564 & 6 & 1 & 65 & 1 & 1 & 1.3 & 0.0 & 0.0 & 0.0 & 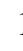 & 1 \\
\hline 51248 & 6 & 1 & 64 & 1 & 2 & 1.0 & 0.0 & 0.0 & 0.0 & 1 & 1 \\
\hline 38326 & 5 & 1 & 64 & 1 & 1 & 1.1 & 0.0 & 0.0 & 0.0 & 1 & 1 \\
\hline 23336 & 4 & 1 & 63 & 2 & 2 & 1.4 & 0.0 & 0.0 & 0.0 & 1 & 1 \\
\hline 30836 & 5 & 1 & 62 & 1 & 2 & 1.3 & 0.0 & 0.0 & 0.0 & 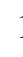 & 1 \\
\hline 24946 & 5 & 1 & 62 & 2 & 3 & 2.2 & 0.0 & 0.0 & 0.0 & 1 & 1 \\
\hline 17800 & 8 & 1 & 61 & 1 & 1 & 0.9 & 0.0 & 0.0 & 0.0 & 1 & 1 \\
\hline 32371 & 5 & 1 & 60 & 1 & 1 & 0.7 & 0.0 & 0.0 & 0.0 & 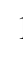 & 1 \\
\hline 117941 & 5 & 1 & 60 & 2 & 2 & 1.2 & 0.0 & 0.0 & 0.0 & 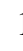 & 1 \\
\hline 49533 & 6 & 1 & 60 & 1 & 1 & 1.2 & 0.0 & 0.0 & 0.0 & 1 & 1 \\
\hline 104713 & 9 & 1 & 59 & 1 & 1 & 1.0 & 0.0 & 0.2 & 0.5 & 2 & 2 \\
\hline 10796 & 5 & 1 & 59 & 1 & 1 & 1.8 & 0.0 & 0.0 & 0.0 & 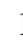 & 1 \\
\hline 44904 & 5 & 1 & 59 & 1 & 1 & 1.8 & 0.0 & 0.0 & 0.0 & 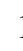 & 1 \\
\hline 31411 & 5 & 1 & 59 & 2 & 3 & 1.2 & 0.0 & 0.0 & 0.0 & 1 & 1 \\
\hline 29430 & 8 & 1 & 57 & 1 & 1 & 0.7 & 0.7 & 0.2 & 0.3 & 2 & 2 \\
\hline 48655 & 5 & 1 & 57 & 2 & 1 & 1.3 & 0.0 & 0.0 & 0.0 & 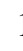 & 1 \\
\hline 12429 & 6 & 1 & 57 & 3 & 1 & 0.9 & 0.0 & 0.0 & 0.0 & 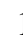 & 1 \\
\hline 30883 & 8 & 1 & 56 & 1 & 2 & 1.0 & 0.1 & 0.2 & 0.3 & 2 & 4 \\
\hline 13688 & 6 & 1 & 55 & 1 & 2 & 1.0 & 0.1 & 0.2 & 0.3 & 2 & 4 \\
\hline
\end{tabular}


EGF containing fibulin-like

extracellular matrix protein 1

Prosaas

Alpha-2-antiplasmin precursor

Inter-alpha-trypsin inhibitor;

Haptoglobin beta chain $\{\mathrm{N}-$

terminal $\}$

Semaphorin-7A isoform 1

preproprotein

Insulin-like growth factor binding

protein-6

Cadherin-2; Neural cadherin;

Complement C2; C3/C5 convertase

Retinoic acid receptor responder

protein 2

Chain a, crystal structure analysis

of the bovine npc2 (niemann-pick

c2) protein

Chain A, Crystal Structure Of A

Human Igm Rheumatoid Factor

Fab In Complex With Its

Autoantigen igg

Insulin-like growth factor-binding

protein 4 precursor

Fibrinogen, gamma A chain

Protein disulfide isomerase

Glutathione S-transferase P

Cell adhesion molecule 4 precursor

Complement component 3

Adipsin/complement factor D

Pro-neuropeptide $Y$

Pyruvate kinase isozymes M1/M2

isoform a

Immunoglobulin V lambda chain

5.1 .11

Immunoglobulin V lambda chain

5.1 .5

\begin{tabular}{|c|c|c|c|c|c|c|c|c|c|c|c|}
\hline 58743 & 5 & 1 & 55 & 2 & 2 & 1.5 & 0.0 & 0.0 & 0.0 & 1 & 1 \\
\hline 27747 & 6 & 1 & 55 & 2 & 2 & 1.9 & 0.0 & 0.0 & 0.0 & 1 & 1 \\
\hline 58364 & 5 & 1 & 55 & 1 & 1 & 1.0 & 0.0 & 0.0 & 0.0 & 1 & 1 \\
\hline 15382 & 6 & 1 & 55 & 1 & 1 & 1.2 & 0.0 & 0.0 & 0.0 & 1 & 1 \\
\hline 2452 & 9 & 1 & 54 & 1 & 1 & 1.7 & 0.0 & 0.0 & 0.0 & 1 & 1 \\
\hline 79541 & 8 & 1 & 54 & 1 & 1 & 1.1 & 0.0 & 0.0 & 0.0 & 1 & 1 \\
\hline 10490 & 10 & 1 & 53 & 0 & 1 & 1.0 & 0.0 & 0.0 & 0.0 & 1 & 1 \\
\hline 105324 & 5 & 1 & 53 & 1 & 2 & 1.3 & 0.0 & 0.0 & 0.0 & 1 & 1 \\
\hline 89708 & 7 & 1 & 52 & 1 & 1 & 1.0 & 0.0 & 0.0 & 0.0 & 1 & 1 \\
\hline 21276 & 9 & 1 & 52 & 1 & 1 & 1.1 & 0.0 & 0.0 & 0.0 & 1 & 1 \\
\hline 16756 & 8 & 1 & 51 & 1 & 1 & 1.1 & 0.0 & 0.0 & 0.0 & 1 & 1 \\
\hline 26076 & 7 & 1 & 51 & 1 & 2 & 1.9 & 0.0 & 0.0 & 0.0 & 1 & 1 \\
\hline 30999 & 7 & 1 & 51 & 1 & 1 & 0.9 & 0.0 & 0.0 & 0.0 & 1 & 1 \\
\hline 39301 & 6 & 1 & 50 & 1 & 2 & 1.2 & 0.0 & 0.0 & 0.0 & 1 & 1 \\
\hline 64027 & 6 & 1 & 50 & 1 & 1 & 1.0 & 0.0 & 0.0 & 0.0 & 1 & 1 \\
\hline 25625 & 7 & 1 & 50 & 1 & 1 & 1.4 & 0.0 & 0.0 & 0.0 & 1 & 1 \\
\hline 44712 & 6 & 1 & 49 & 1 & 1 & 0.9 & 0.0 & 0.0 & 0.0 & 1 & 1 \\
\hline 8803 & 5 & 1 & 49 & 1 & 1 & 1.2 & 0.0 & 0.0 & 0.0 & 1 & 1 \\
\hline 22043 & 6 & 1 & 49 & 2 & 2 & 1.3 & 0.0 & 0.0 & 0.0 & 1 & 1 \\
\hline 9115 & 5 & 1 & 49 & 1 & 2 & 1.8 & 0.0 & 0.0 & 0.0 & 1 & 1 \\
\hline 63836 & 8 & 1 & 48 & 1 & 1 & 1.9 & 0.0 & 0.0 & 0.0 & 1 & 1 \\
\hline 11278 & 6 & 1 & 48 & 2 & 1 & 1.5 & 0.0 & 0.0 & 0.0 & 1 & 1 \\
\hline 11332 & 5 & 1 & 48 & 2 & 1 & 1.5 & 0.0 & 0.0 & 0.0 & 1 & 1 \\
\hline
\end{tabular}


Igg3 heavy chain constant region

Nidogen-2

Ig mu heavy chain $\mathrm{V}$ region

precursor - sheep (fragment)

Thrombospondin-2 precursor

Neuroendocrine protein 7B2

isoform 1

SPARC-like protein 1 precursor

C4b-binding protein alpha chain

precursor

Protein C prepropeptide

Alpha globin chain

Galectin 1

Neuroblastoma suppressor of

tumorigenicity 1 precursor

Plasma glutamate carboxypeptidase

precursor

Aspartate aminotransferase,

cytoplasmic

Repulsive guidance molecule A isoform 3

14-3-3 protein gamma; Protein

kinase $\mathrm{C}$ inhibitor protein 1

CD166 antigen; Activated

leukocyte cell adhesion molecule; altname: Ful

Serum paraoxonase/arylesterase 1

Cathepsin L2 precursor

Unnamed protein product

Complement C4B precursor

Malate dehydrogenase,

cytoplasmic isoform 2

Mimecan; osteoglycin

Alpha-1-antiproteinase; Alpha-1-

antitrypsin; Alpha-1-prot

Complement component C6; Flags:

Precursor

\begin{tabular}{|c|c|c|c|c|c|c|c|c|c|c|c|}
\hline 42669 & 8 & 1 & 47 & 1 & 1 & 1.2 & 0.0 & 0.0 & 0.0 & 1 & 1 \\
\hline 158020 & 5 & 1 & 47 & 2 & 2 & 0.8 & 0.0 & 0.0 & 0.0 & 1 & 1 \\
\hline 15956 & 9 & 1 & 47 & 1 & 1 & 1.1 & 0.0 & 0.0 & 0.0 & 1 & 1 \\
\hline 138958 & 5 & 1 & 47 & 1 & 2 & 1.0 & 0.0 & 0.0 & 0.0 & 1 & 1 \\
\hline 25536 & 6 & 1 & 47 & 1 & 3 & 3.0 & 0.0 & 0.0 & 0.0 & 1 & 1 \\
\hline 77718 & 5 & 1 & 47 & 1 & 2 & 1.8 & 0.0 & 0.0 & 0.0 & 1 & 1 \\
\hline 76307 & 6 & 1 & 46 & 1 & 1 & 1.2 & 0.0 & 0.0 & 0.0 & 1 & 1 \\
\hline 55951 & 6 & 1 & 46 & 1 & 1 & 0.8 & 0.0 & 0.0 & 0.0 & 1 & 1 \\
\hline 16968 & 9 & 1 & 46 & 1 & 2 & 2.2 & 0.0 & 0.0 & 0.0 & 1 & 1 \\
\hline 16381 & 5 & 1 & 45 & 2 & 2 & 1.6 & 0.0 & 0.0 & 0.0 & 1 & 1 \\
\hline 20837 & 5 & 1 & 45 & 1 & 2 & 1.6 & 0.0 & 0.0 & 0.0 & 1 & 1 \\
\hline 55474 & 6 & 1 & 45 & 2 & 2 & 1.2 & 0.0 & 0.0 & 0.0 & 1 & 1 \\
\hline 49640 & 7 & 1 & 44 & 2 & 1 & 1.4 & 0.0 & 0.0 & 0.0 & 1 & 1 \\
\hline 53091 & 7 & 1 & 44 & 1 & 1 & 1.0 & 0.0 & 0.0 & 0.0 & 1 & 1 \\
\hline 30967 & 5 & 1 & 44 & 1 & 2 & 1.3 & 0.0 & 0.0 & 0.0 & 1 & 1 \\
\hline 64831 & 6 & 1 & 44 & 1 & 1 & 1.1 & 0.0 & 0.0 & 0.0 & 1 & 1 \\
\hline 43725 & 6 & 1 & 43 & 1 & 1 & 2.6 & 0.0 & 0.0 & 0.0 & 1 & 1 \\
\hline 41703 & 7 & 1 & 43 & 1 & 1 & 1.0 & 0.0 & 0.0 & 0.0 & 1 & 1 \\
\hline 46412 & 5 & 1 & 43 & 1 & 1 & 0.5 & 0.0 & 0.0 & 0.0 & 1 & 1 \\
\hline 200574 & 7 & 1 & 42 & 1 & 2 & 1.9 & 0.0 & 0.0 & 0.0 & 1 & 1 \\
\hline 41198 & 7 & 1 & 42 & 1 & 1 & 1.2 & 0.0 & 0.0 & 0.0 & 1 & 1 \\
\hline 38499 & 5 & 1 & 42 & 1 & 1 & 1.1 & 0.0 & 0.0 & 0.0 & 1 & 1 \\
\hline 50821 & 6 & 1 & 41 & 0 & 0 & 0.5 & 0.0 & 0.0 & 0.0 & 1 & 1 \\
\hline 117535 & 6 & 1 & 41 & 1 & 2 & 1.2 & 0.0 & 0.0 & 0.0 & 1 & 1 \\
\hline
\end{tabular}


Ig lambda chain C region - horse (fragment)

Kininogen-1 isoform 2

KIAA0578 protein

Fibromodulin

Neurocan core protein precursor

[Pan troglodytes]

Endopin 2B

Complement c1r-A subcomponent

precursor

Lysosomal alpha-mannosidase

Immunoglobulin light chain

variable region

Complement component 3

Serum amyloid A protein

Unnamed protein product

Glutathione S-transferase Mu 1

Coagulation factor IX

Presequence protease

mitochondrial isoform 2 precursor

Immunoglobulin igg-2 heavy chain

constant region

Secreted nidogen domain protein

precursor

Protein kinase C-binding protein

NELL2

Chain a, new crystal forms of a mu class glutathione s-transferase

Fibrous sheath interacting protein 1

Chain a, crystal structure of

tetradeca-(3-fluorotyrosyl)-

glutathione s-transferase

EGF-containing fibulin-like

extracellular matrix protein 2

Fibulin-4;

Complement component C7

precursor

\begin{tabular}{|c|c|c|c|c|c|c|c|c|c|c|c|}
\hline 13899 & 8 & 1 & 41 & 1 & 2 & 1.5 & 0.0 & 0.0 & 0.0 & 1 & 1 \\
\hline 53513 & 6 & 1 & 41 & 1 & 1 & 1.3 & 0.0 & 0.0 & 0.0 & 1 & 1 \\
\hline 182933 & 6 & 1 & 39 & 1 & 1 & 0.8 & 0.0 & 0.0 & 0.0 & 1 & 1 \\
\hline 44986 & 6 & 1 & 40 & 1 & 1 & 1.2 & 0.0 & 0.0 & 0.0 & 1 & 1 \\
\hline 149096 & 5 & 1 & 40 & 1 & 1 & 0.7 & 0.0 & 0.0 & 0.0 & 1 & 1 \\
\hline 51047 & 6 & 1 & 40 & 1 & 1 & 1.1 & 0.0 & 0.0 & 0.0 & 1 & 1 \\
\hline 86405 & 5 & 1 & 40 & 1 & 1 & 1.7 & 0.0 & 0.0 & 0.0 & 1 & 1 \\
\hline 117756 & 7 & 1 & 40 & 1 & 1 & 1.2 & 0.0 & 0.0 & 0.0 & 1 & 1 \\
\hline 11310 & 6 & 1 & 40 & 2 & 2 & 1.5 & 0.0 & 0.0 & 0.0 & 1 & 1 \\
\hline 18508 & 5 & 1 & 40 & 1 & 1 & 1.2 & 0.0 & 0.0 & 0.0 & 1 & 1 \\
\hline 8367 & 6 & 1 & 40 & 1 & 1 & 1.2 & 0.0 & 0.0 & 0.0 & 1 & 1 \\
\hline 52913 & 5 & 1 & 40 & 2 & 1 & 1.0 & 0.0 & 0.0 & 0.0 & 1 & 1 \\
\hline 28494 & 7 & 1 & 40 & 1 & 1 & 2.3 & 0.0 & 0.0 & 0.0 & 1 & 1 \\
\hline 52234 & 5 & 1 & 40 & 1 & 1 & 1.0 & 0.0 & 0.0 & 0.0 & 1 & 1 \\
\hline 127532 & 6 & 1 & 40 & 1 & 1 & 0.7 & 0.0 & 0.0 & 0.0 & 1 & 1 \\
\hline 46351 & 6 & 1 & 40 & 2 & 1 & 1.3 & 0.0 & 0.0 & 0.0 & 1 & 1 \\
\hline 161194 & 6 & 1 & 39 & 2 & 1 & 1.5 & 0.0 & 0.0 & 0.0 & 1 & 1 \\
\hline 100049 & 5 & 1 & 39 & 2 & 2 & 1.2 & 0.0 & 0.0 & 0.0 & 1 & 1 \\
\hline 28786 & 8 & 1 & 39 & 2 & 3 & 3.2 & 0.0 & 0.0 & 0.0 & 1 & 1 \\
\hline 75849 & 5 & 1 & 39 & 0 & 0 & 0.9 & 0.0 & 0.0 & 0.0 & 1 & 1 \\
\hline 28109 & 9 & 1 & 38 & 1 & 1 & 2.3 & 0.0 & 0.0 & 0.0 & 1 & 1 \\
\hline 51861 & 5 & 1 & 38 & 1 & 1 & 1.2 & 0.0 & 0.0 & 0.0 & 1 & 1 \\
\hline 103094 & 6 & 1 & 38 & 1 & 1 & 1.2 & 0.0 & 0.0 & 0.0 & 1 & 1 \\
\hline
\end{tabular}




\begin{tabular}{|c|c|c|c|c|c|c|c|c|c|c|c|c|c|}
\hline Secretogranin-2 precursor & 76942 & 5 & 1 & 38 & 1 & 2 & 1.1 & 0.0 & 0.0 & 0.0 & 1 & 1 & 1 \\
\hline Complement C8 beta & 17458 & 8 & 1 & 38 & 1 & 1 & 0.9 & 0.0 & 0.0 & 0.0 & 1 & 1 & 1 \\
\hline Histone deacetylase 7 & 28292 & 10 & 1 & 38 & 5 & 2 & 0.9 & 0.0 & 0.0 & 0.0 & 1 & 1 & 1 \\
\hline $\begin{array}{l}\text { Complement factor B subunit } \mathrm{Bb} \text { - } \\
\text { pig (fragment) }\end{array}$ & 21793 & 6 & 1 & 38 & 1 & 2 & 1.5 & 0.0 & 0.0 & 0.0 & 1 & 1 & 1 \\
\hline $\begin{array}{l}\text { Nuclear factor of activated T-cells, } \\
\text { cytoplasmic } 2 \text { isoform C }\end{array}$ & 105863 & 7 & 1 & 38 & 1 & 2 & 0.4 & 0.0 & 0.0 & 0.0 & 1 & 1 & 1 \\
\hline Fibroleukin & 55998 & 8 & 1 & 38 & 2 & 2 & 1.4 & 0.0 & 0.0 & 0.0 & 1 & 1 & 1 \\
\hline Fibulin-5 precursor & 52687 & 5 & 1 & 38 & 2 & 2 & 1.3 & 0.0 & 0.0 & 0.0 & 1 & 1 & 1 \\
\hline $\begin{array}{l}\text { Ig lambda chain V region } \\
\text { Precursor polypeptide (AA -19 to }\end{array}$ & 12660 & 9 & 1 & 38 & 1 & 1 & 1.1 & 0.0 & 0.0 & 0.0 & 1 & 1 & 1 \\
\hline 206) & 28103 & 6 & 1 & 38 & 1 & 1 & 1.4 & 0.0 & 0.0 & 0.0 & 1 & 1 & 1 \\
\hline
\end{tabular}

Molecular weight (MW) is predicted based on amino acid sequence. 


\section{Applied Biosystems}

Analysis Information

Report Type Protein-Peptide Summary by Sample Set

Sample Set Name ITRAQ Sept11

Analysis Type

Database

MS/MS

95\% Cl pep rank1 NCBInr

Creation Date

NewNCBInr

Reported By 11/01/2011 10:22:25 - admin

Last Modified

10/31/2011 11:18:48

10/31/2011 20:00:34

MS Acq. : Proc. Methods (Unspecified) : (Unspecified)

Interpretation Method (Unspecified)

\begin{tabular}{|c|c|c|c|c|c|c|c|c|c|c|c|c|c|c|c|}
\hline Rank & Protein Name & Accession No. & Protein MW & $\begin{array}{c}\text { Pep. } \\
\text { Count }\end{array}$ & $\begin{array}{r}\text { Total lon } \\
\text { Score }\end{array}$ & $\begin{array}{r}\text { Avg. } \\
\text { iTRAQ } \\
\text { Ratio } \\
114 / 117^{*}\end{array}$ & $\begin{array}{r}\text { Avg. } \\
\text { iTRAQ } \\
\text { Ratio } \\
115 / 117^{*}\end{array}$ & $\begin{array}{r}\text { Avg. } \\
\text { iTRAQ } \\
\text { Ratio } \\
116 / 117^{*}\end{array}$ & $\begin{array}{r}\text { iTRAQ } \\
\text { SDev } \\
114 / 117^{*}\end{array}$ & $\begin{array}{r}\text { iTRAQ } \\
\text { SDev. } \\
115 / 117^{*}\end{array}$ & $\begin{array}{r}\text { iTRAQ } \\
\text { SDev. } \\
116 / 117^{*}\end{array}$ & $\begin{array}{r}\text { iTRAQ } \\
\text { Peptides } \\
114 / 117\end{array}$ & $\begin{array}{r}\text { iTRAQ } \\
\text { Peptides } \\
115 / 117\end{array}$ & $\begin{array}{r}\text { iTRAQ } \\
\text { Peptides } \\
116 / 117\end{array}$ & $\begin{array}{l}\text { Total lon } \\
\text { C. I. \% }\end{array}$ \\
\hline & serum albumin precursor [Ovis aries] & gi|57164373 & 79687.2 & 43 & 2879 & 0.989 & 1.113 & 0.950 & 0.493 & 0.470 & 0.244 & 147 & 147 & 147 & 100 \\
\hline
\end{tabular}

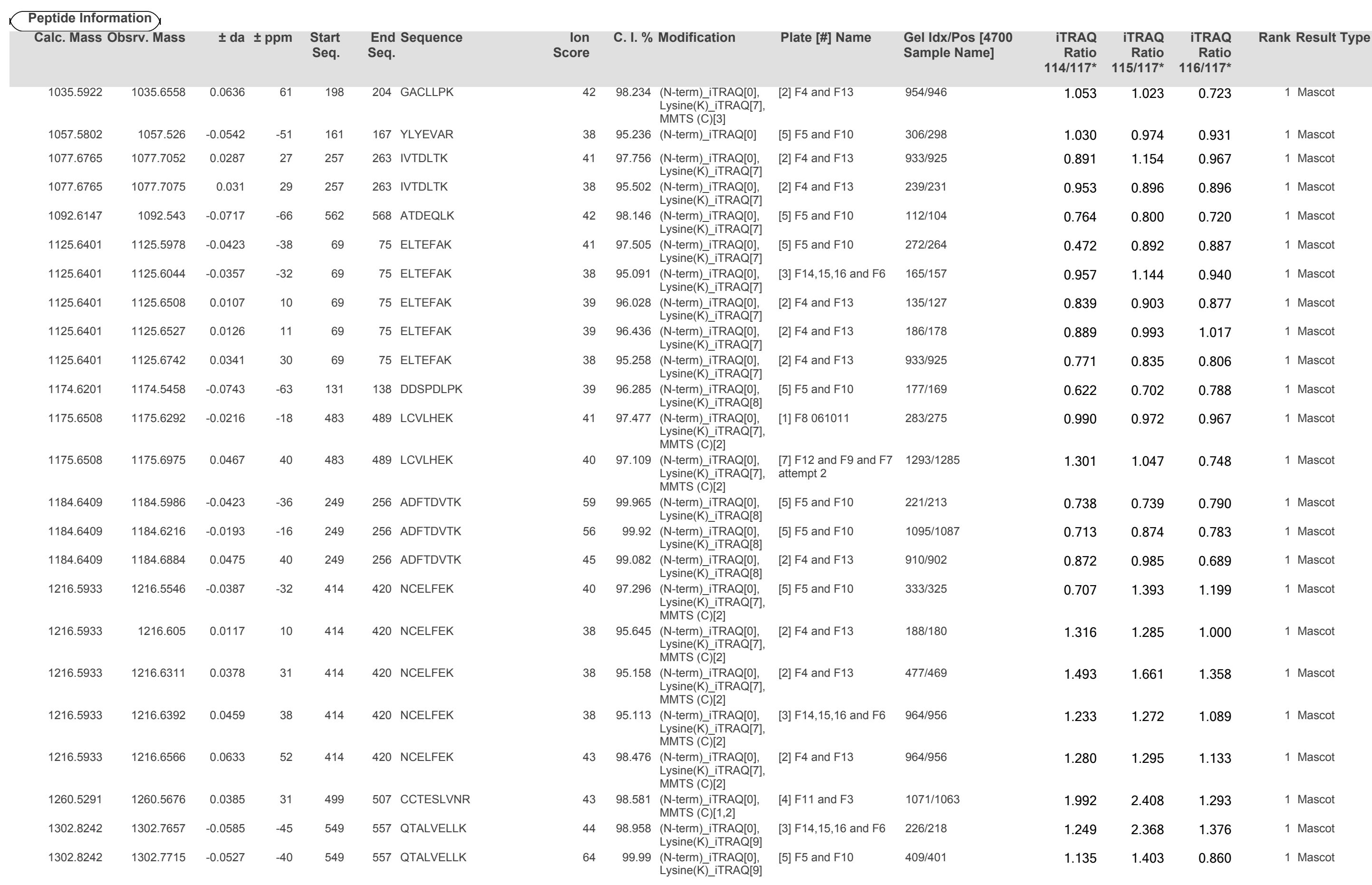




\begin{tabular}{|c|c|c|c|c|c|}
\hline 302.8242 & 1302.8359 & 0.0117 & 9 & 549 & 557 QTALVELLK \\
\hline 302.8242 & 1302.8368 & 0.0126 & 10 & 549 & 557 QTALVELLK \\
\hline 1302.8242 & 1302.8618 & 0.0376 & 29 & 549 & 557 QTALVELLK \\
\hline 1335.6627 & 1335.6188 & -0.0439 & -33 & 310 & 318 SHCIAEVDK \\
\hline 338.6526 & 1338.6294 & -0.0232 & -17 & 123 & 130 NECFLNHK \\
\hline 412.7145 & 1412.7344 & 0.0199 & 14 & 588 & 597 EGCFVLEGPK \\
\hline 1412.7145 & 1412.757 & 0.0425 & 30 & 588 & 597 EGCFVLEGPK \\
\hline 1427.8131 & 1427.851 & 0.0379 & 27 & 361 & 371 HPEYAVSVLLR \\
\hline 1488.7906 & 1488.8635 & 0.0729 & 49 & 413 & 420 KNCELFEK \\
\hline 1488.7906 & 1488.8796 & 0.089 & 60 & 413 & 420 KNCELFEK \\
\hline 1575.0216 & 1574.9834 & -0.0382 & -24 & 548 & 557 KQTALVELLK \\
\hline 1575.0216 & 1575.0242 & 0.0026 & 2 & 548 & 557 KQTALVELLK \\
\hline 1575.0216 & 1575.1365 & 0.1149 & 73 & 548 & 557 KQTALVELLK \\
\hline 1593.921 & 1593.8564 & -0.0646 & -41 & 402 & 412 HLVDEPQNLIK \\
\hline 1593.921 & 1593.8634 & -0.0576 & -36 & 402 & 412 HLVDEPQNLIK \\
\hline 1593.921 & 1593.975 & 0.054 & 34 & 402 & 412 HLVDEPQNLIK \\
\hline 1593.921 & 1593.9929 & 0.0719 & 45 & 402 & 412 HLVDEPQNLIK \\
\hline 1593.921 & 1594.0195 & 0.0985 & 62 & 402 & 412 HLVDEPQNLIK \\
\hline 1609.99 & 1610.0524 & 0.0624 & 39 & 66 & 75 LVKELTEFAK \\
\hline 1641.9296 & 1641.8838 & -0.0458 & -28 & 438 & $51 \mathrm{APQ}$ \\
\hline 647.8727 & 1647.8531 & -0.0196 & -12 & 421 & 433 HGEYGFQNALIVR \\
\hline 647.8727 & 1647.8931 & 0.0204 & 12 & 421 & 433 HGEYC \\
\hline 1647.8727 & 1647.9025 & 0.0298 & 18 & 421 & 433 HGEYGFQNALIVR \\
\hline 1647.8727 & 1647.9867 & 0.114 & 69 & 421 & 433 HGEYGFQNALIVR \\
\hline 1687.8225 & 1687.7981 & -0.0244 & -14 & 300 & 309 ECCDKPVLEK \\
\hline 1687.8225 & 1687.8889 & 0.0664 & 39 & 300 & 309 ECCDKPVLEK \\
\hline 1687.8975 & 1687.8418 & -0.0557 & -33 & 569 & 580 TVMENFVAFVDK \\
\hline 1687.8975 & 1687.8925 & -0.005 & -3 & 569 & 580 TVMENFVAFVDK \\
\hline 1687.8975 & 1687.9059 & 0.0084 & 5 & 569 & 580 TVMENFVAFVDK \\
\hline 1687.8975 & 1687.9149 & 0.0174 & 10 & 569 & 580 TVMENFVAFVDK \\
\hline 1687.8975 & 1687.9182 & 0.0207 & 12 & 569 & 580 TVMENFVAFVDK \\
\hline 1687.8975 & 1687.9308 & 0.0333 & 20 & 569 & 580 TVMENFVAFVDK \\
\hline 1687.8975 & 1687.9449 & 0.0474 & 28 & 569 & 580 TVMENFVAFVDK \\
\hline 1687.8975 & 1687.9557 & 0.0582 & 34 & 569 & 580 TVMENFVAFVDK \\
\hline 1687.8975 & 1687.9612 & 0.0637 & 38 & 569 & 580 TVMENFVAFVDK \\
\hline 1696.8629 & 1696.818 & -0.0449 & -26 & 89 & 100 SLHTLFGDELCK \\
\hline 1696.8629 & 1696.8333 & -0.0296 & -17 & 89 & 100 SLHTLFGDELCK \\
\hline 596.8629 & 1696.8533 & -0.0096 & & 39 & 10 SLHTLFGDELCF \\
\hline
\end{tabular}

7099.997 (N-term) iTRAQ[0], [4] F11 and F3 1149/1141

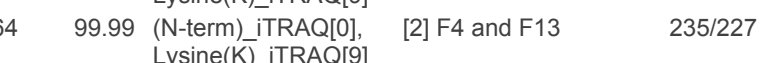

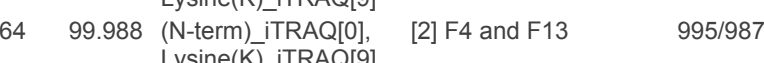

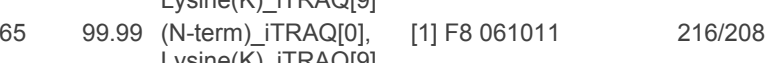
Lysine(K) iTRAQ[9],

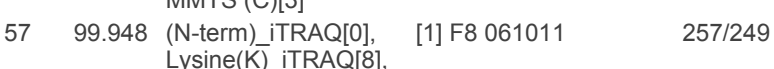
MMTS (C)[3]

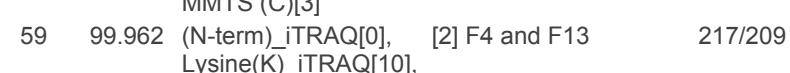

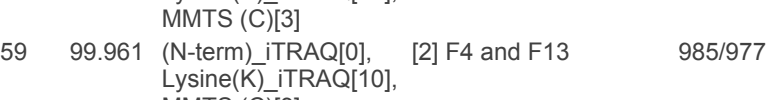
MMTS (C)[3] $[3]$

$41 \quad 97.693$ (N-term)_iTRAQ[0] $\quad[3] \mathrm{F} 14,15,16$ and F6 $\quad 902 / 894$

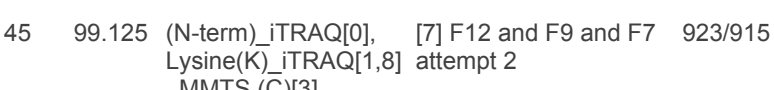

$4298.138 \begin{aligned} & \text { MMTS (C)[3] } \\ & \text { (N-term) iTRAC[0], } \\ & \text { Lysine(K) } \text { iTRAQ[1,8] attempt 2 }\end{aligned}$

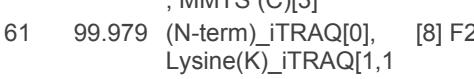

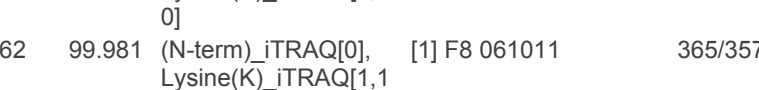

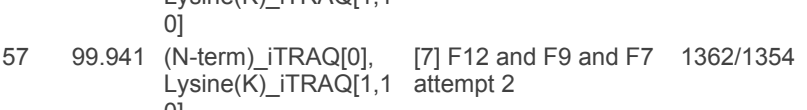

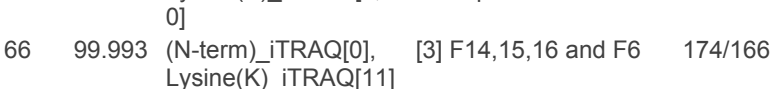

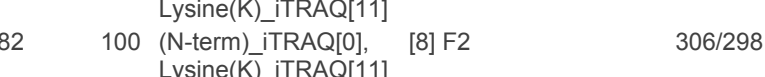

80100 (N-term) iTRAQ[0], [3] F14,15,16 and F6 $821 / 813$

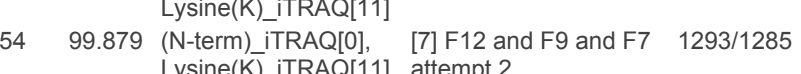

$\begin{array}{ll}62 & 99.983 \\ \text { (N-term) iTRAQ[0], } \\ \text { Lysine }(K) \text { iTRAQ[1] }\end{array}$

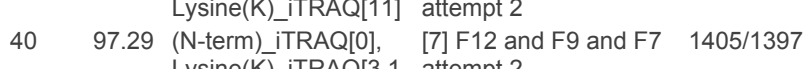

$\begin{array}{llll}0 & 01 \\ 65 & 99.99(\mathrm{~N} \text {-term)_iTRAQ[0] } & {[8] \mathrm{F} 2} & 352 / 344\end{array}$

$60 \quad 99.974$ (N-term)_iTRAQ[0] [3] F14,15,16 and F6 $\quad 203 / 195$

4599.06 (N-term)_iTRAQ[0] [4] F11 and F3 $\quad 1100 / 1092$

$58 \quad 99.957$ (N-term)_iTRAQ[0] [5] F5 and F10 $1227 / 1219$

5999.967 (N-term)_iTRAQ[0] [3] F14,15,16 and F6 $867 / 859$

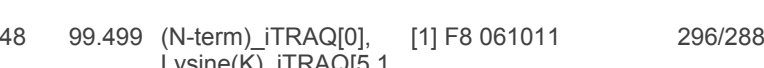

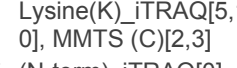

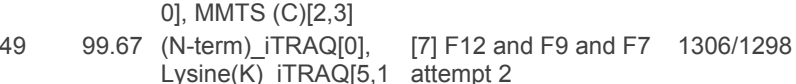

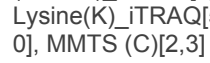

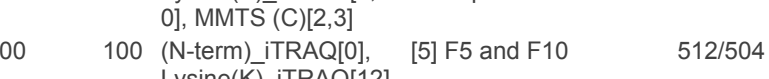

6799.994 (N-term) ITRAA[0], [1] F8 061011 501/493

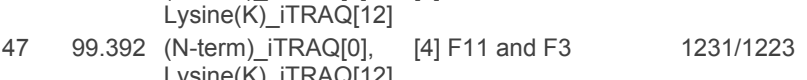

90100 (N-term) iTRAQ[0] ] [5] F5 and F10 1382/1374

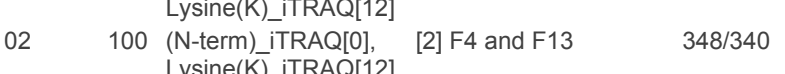

100 (N-term) ITRAC[0], [4] F11 and F3 1272/1264

4999.662 (N-term) iTRAQ[0], [3] F14,15,16 and F6 1048/1040

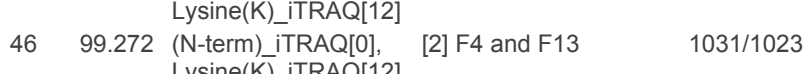

$53 \quad 99.852$ (N-term) iTRAQ[0], [7] F12 and F9 and F7 1084/1076

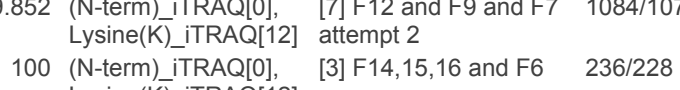
Lysien(K) $(T R A Q[1]$
MMTS (C) [11]

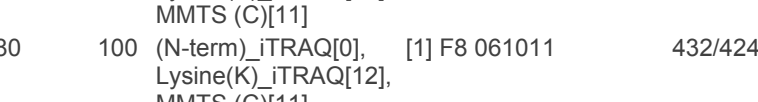

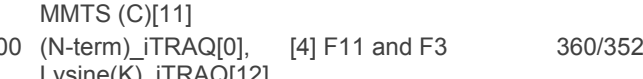

\begin{tabular}{|c|c|c|c|}
\hline 1.196 & 1.443 & 0.987 & 1 Mascot \\
\hline 0.663 & 1.379 & 1.131 & 1 Mascot \\
\hline 0.751 & 1.218 & 1.051 & 1 Mascot \\
\hline 0.960 & 1.116 & 0.923 & 1 Mascot \\
\hline 0.935 & 0.980 & 0.912 & 1 Mascot \\
\hline 0.761 & 0.660 & 0.721 & 1 Mascot \\
\hline 0.784 & 0.952 & 0.841 & 1 Mascot \\
\hline 0.682 & 0.665 & 0.694 & 1 Mascot \\
\hline 0.767 & 0.779 & 0.859 & 1 Mascot \\
\hline 0.728 & 0.912 & 0.869 & 1 Mascot \\
\hline 1.065 & 1.281 & 1.002 & 1 Mascot \\
\hline 0.570 & 1.183 & 1.125 & 1 Mascot \\
\hline 0.912 & 0.993 & 0.968 & 1 Mascot \\
\hline 1.536 & 1.441 & 0.941 & 1 Mascot \\
\hline 0.873 & 0.928 & 0.888 & 1 Mascot \\
\hline 1.128 & 0.994 & 0.937 & 1 Mascot \\
\hline 0.851 & 0.864 & 0.846 & 1 Mascot \\
\hline 1.204 & 1.723 & 1.246 & 1 Mascot \\
\hline 0.894 & 1.263 & 0.966 & 1 Mascot \\
\hline 0.666 & 0.756 & 0.734 & 1 Mascot \\
\hline 0.917 & 1.114 & 0.986 & 1 Mascot \\
\hline 1.575 & 1.505 & 1.070 & 1 Mascot \\
\hline 1.821 & 2.149 & 1.349 & 1 Mascot \\
\hline 1.046 & 0.896 & 0.933 & 1 Mascot \\
\hline 0.704 & 0.888 & 1.066 & 1 Mascot \\
\hline 0.897 & 0.911 & 0.842 & 1 Mascot \\
\hline 1.113 & 1.245 & 0.975 & 1 Mascot \\
\hline 0.506 & 1.255 & 0.753 & 1 Mascot \\
\hline 0.887 & 1.062 & 0.891 & 1 Mascot \\
\hline 1.453 & 1.560 & 0.727 & 1 Mascot \\
\hline 0.914 & 1.109 & 0.881 & 1 Mascot \\
\hline 1.120 & 1.121 & 0.952 & 1 Mascot \\
\hline 1.092 & 0.966 & 1.058 & 1 Mascot \\
\hline 1.939 & 1.118 & 2.089 & 1 Mascot \\
\hline 1.666 & 1.429 & 1.798 & 1 Mascot \\
\hline 0.515 & 0.534 & 0.670 & 1 Mascot \\
\hline 0.417 & 0.545 & 0.730 & 1 Mascot \\
\hline & 0.549 & 0.62 & \\
\hline
\end{tabular}




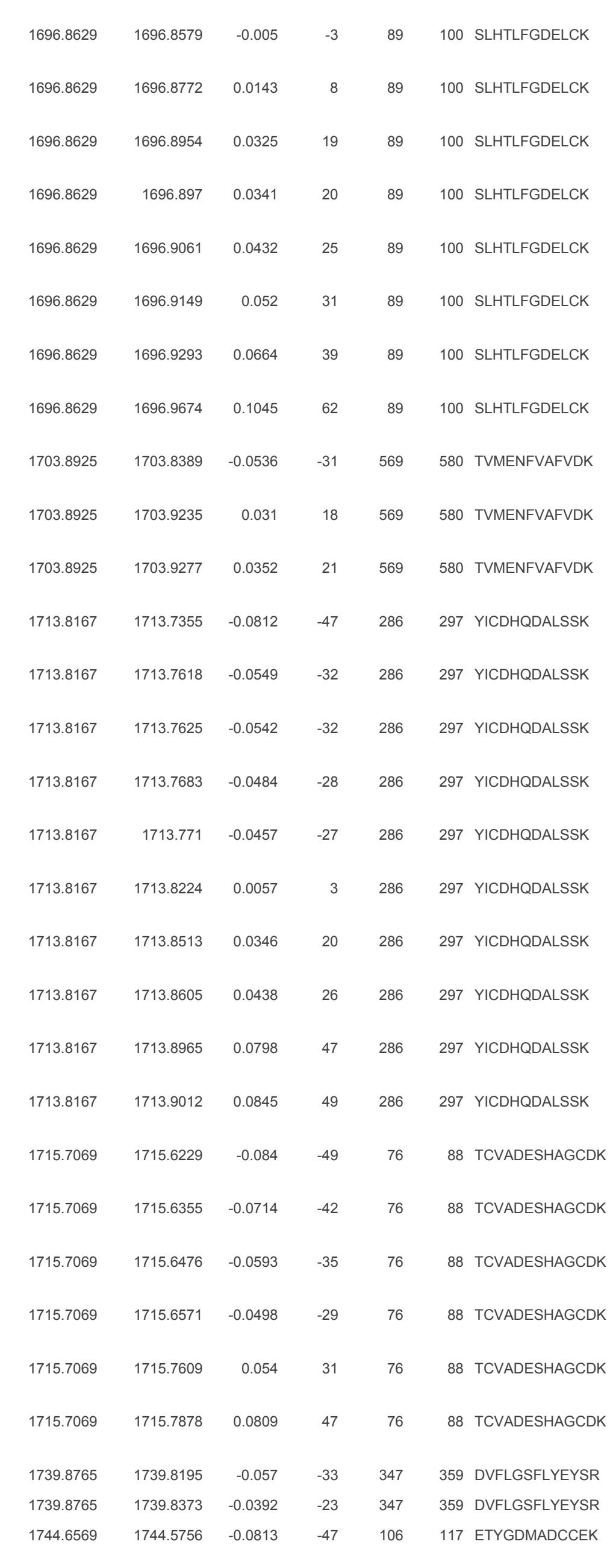

45 MMTS (C)[11]

(11] F8 $061011 \quad 467 / 459$

MMTS (C)[11] 12$]$,
(N-term) iTRAQ[0], [5] F5 and F10 1298/1290

(N-term) iTRAQ[0],
Lysine(K)

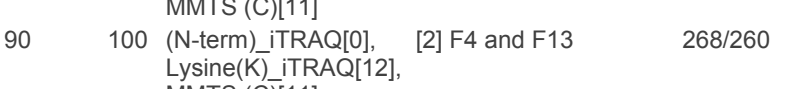

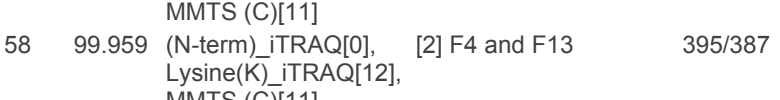

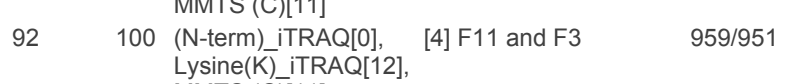

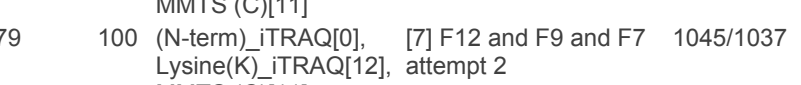

95100 (N-term) iTRAQ[0], [3] F14, 15,16 and F6 963/955

100 (N-term) iTRAQ[0],
Lysine(K) iRAQ[12],
MMTS (C) (111]

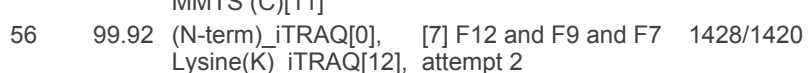

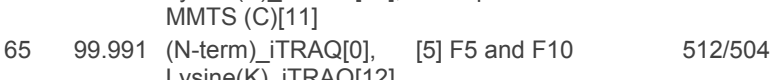

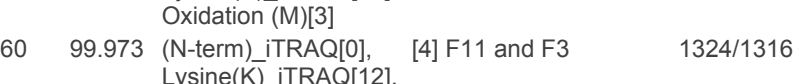

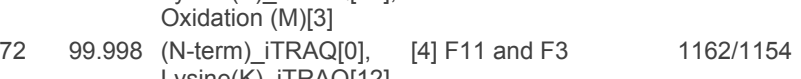

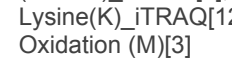

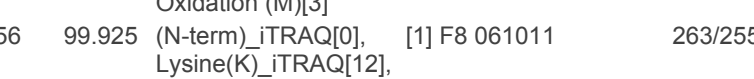

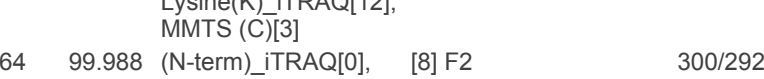

99.988 (N-term) iTRAQ[0],
Lysine(k) iTRAQ[12],
LMTS F2

$300 / 292$

00 (N-term) iTRAQ[0],
Lysine(K)_iTRAQ[12],

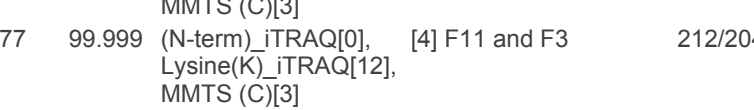

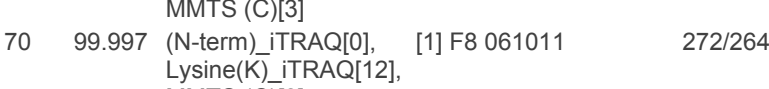

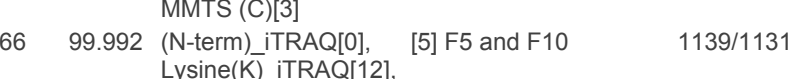

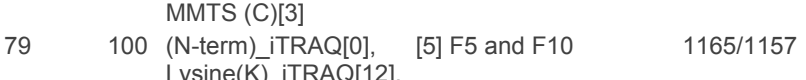

$100(\mathrm{~N}$-term) iTRAQ[0],
Lsine(K) Lysine(K)
MMTS (C) [I] 3 RA

$46 \quad 99.302$ (N-term) iTRAQ[0], [7] F12 and F9 and F7 $1287 / 1279$ Lysine(K) iTRAQ
MMTS (C)[3]

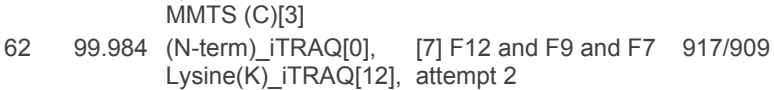
Lysine(K) $(\mathrm{iTR})$
MMTS (C) [3]

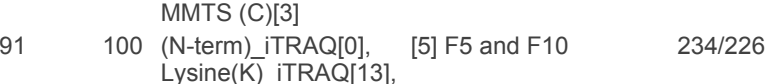

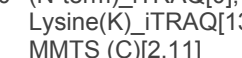

99100 (N-term) itRAQ[0], [8] F2 240/232

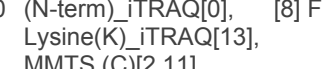

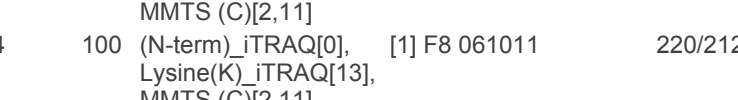

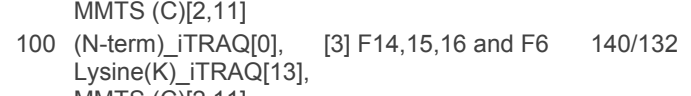

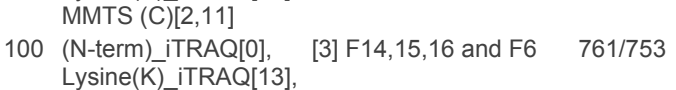

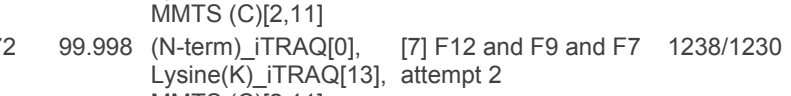
MMTS (C) $[2,11]$
100 (N-term) iTRAQ[0] [8] F2

$528 / 520$

6999.997 (N-term)_iTRAQ[0] [5] F5 and F10 $539 / 531$

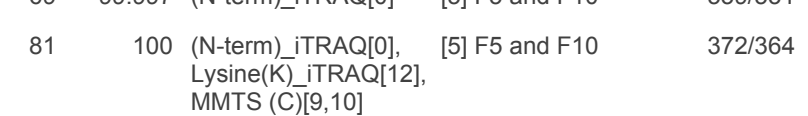

\begin{tabular}{|c|c|c|c|}
\hline 0.333 & 0.594 & 0.650 & 1 Mascot \\
\hline 1.501 & 2.002 & 1.278 & 1 Mascot \\
\hline 0.740 & 1.016 & 1.053 & 1 Mascot \\
\hline 1.702 & 1.957 & 2.371 & 1 Mascot \\
\hline 0.444 & 0.630 & 0.658 & 1 Mascot \\
\hline 1.127 & 1.204 & 1.089 & 1 Mascot \\
\hline 0.565 & 0.588 & 0.718 & 1 Mascot \\
\hline 0.293 & 0.582 & 0.761 & 1 Mascot \\
\hline 0.947 & 0.682 & 0.857 & 1 Mascot \\
\hline 0.981 & 1.153 & 0.881 & 1 Mascot \\
\hline 1.040 & 1.306 & 1.265 & 1 Mascot \\
\hline 1.856 & 1.835 & 1.293 & 1 Mascot \\
\hline 0.598 & 0.783 & 0.774 & 1 Mascot \\
\hline 0.750 & 0.756 & 0.775 & 1 Mascot \\
\hline 0.976 & 1.094 & 0.818 & 1 Mascot \\
\hline 0.596 & 0.815 & 0.827 & 1 Mascot \\
\hline 1.128 & 1.501 & 0.763 & 1 Mascot \\
\hline 1.397 & 1.609 & 1.181 & 1 Mascot \\
\hline 0.839 & 0.761 & 0.818 & 1 Mascot \\
\hline 0.563 & 0.829 & 0.901 & 1 Mascot \\
\hline 1.249 & 1.693 & 1.438 & 1 Mascot \\
\hline 1.468 & 1.953 & 1.384 & 1 Mascot \\
\hline 0.910 & 1.195 & 0.815 & 1 Mascot \\
\hline 1.117 & 1.293 & 1.153 & 1 Mascot \\
\hline 1.503 & 1.461 & 0.913 & 1 Mascot \\
\hline 1.157 & 1.099 & 0.900 & 1 Mascot \\
\hline 1.064 & 1.113 & 0.939 & 1 Mascot \\
\hline 0.723 & 0.723 & 0.784 & 1 Mascot \\
\hline 1.208 & 1.740 & 1.228 & 1 Mascot \\
\hline 0.601 & 0.935 & 0.924 & 1 Mascot \\
\hline
\end{tabular}




\begin{tabular}{|c|c|c|c|c|c|c|c|c|c|c|c|c|c|c|}
\hline 1744.6569 & 1744.6769 & 0.02 & 11 & 106 & 117 ETYGDMADCCEK & 85 & 100 & 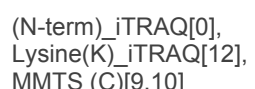 & , ${ }^{[2] ~ F 4 ~ a n d ~ F 13 ~}$ & 188/180 & 0.564 & 1.022 & 0.953 & 1 Mascot \\
\hline 1744.6569 & 1744.6855 & 0.0286 & 16 & 106 & 117 ETYGDMADCCEK & 50 & 99.723 & 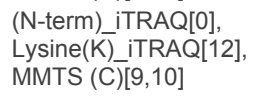 & , ${ }^{[4] ~ F 11 ~ a n d ~ F 3 ~}$ & 1076/1068 & 1.209 & 1.073 & 0.938 & 1 Mascot \\
\hline 1744.6569 & 1744.6956 & 0.0387 & 22 & 106 & 117 ETYGDMADCCEK & 80 & 100 & 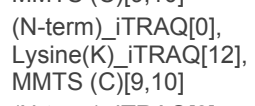 & , ${ }^{[4] F 11 \text { and F3 }}$ & 1110/1102 & 0.855 & 0.800 & 0.763 & 1 Mascot \\
\hline 1744.6569 & 1744.717 & 0.0601 & 34 & 106 & 117 ETYGDMADCCEK & 65 & 99.991 & 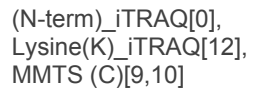 & [2] F4 and F13 & 975/967 & 0.542 & 1.061 & 0.868 & 1 Mascot \\
\hline 1744.6569 & 1744.7797 & 0.1228 & 70 & 106 & 117 ETYGDMADCCEK & 38 & 95.793 & 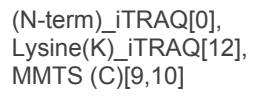 & $\begin{array}{l}\text { [7] F12 and F9 and F7 } \\
\text {, attempt } 2\end{array}$ & $1368 / 1360$ & 1.180 & 1.412 & 0.907 & 1 Mascot \\
\hline 1754.7317 & 1754.6647 & -0.067 & -38 & 375 & 386 EYEATLEDCCAK & 81 & 100 & 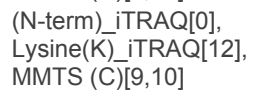 & , $[5]$ F5 and F10 & $434 / 426$ & 1.193 & 1.237 & 0.787 & 1 Mascot \\
\hline 1754.7317 & 1754.7434 & 0.0117 & 7 & 375 & 386 EYEATLEDCCAK & 74 & 99.999 & 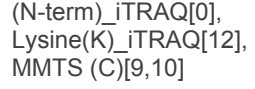 & , $[4]$ F11 and F3 & 1181/1173 & 1.263 & 1.219 & 0.852 & 1 Mascot \\
\hline 1754.7317 & 1754.7574 & 0.0257 & 15 & 375 & 386 EYEATLEDCCAK & 86 & 100 & 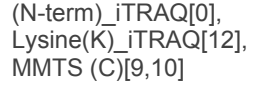 & , [2] F4 and F13 & 265/257 & 1.329 & 1.220 & 0.758 & 1 Mascot \\
\hline 1754.7317 & 1754.8008 & 0.0691 & 39 & 375 & 386 EYEATLEDCCAK & 39 & 96.361 & 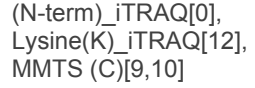 & [3] F14,15,16 and F6 & 961/953 & 1.224 & 1.824 & 1.030 & 1 Mascot \\
\hline 1754.7317 & 1754.8232 & 0.0915 & 52 & 375 & 386 EYEATLEDCCAK & 48 & 99.572 & 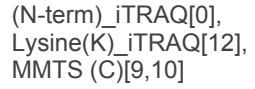 & , [4] F11 and F3 & $1258 / 1250$ & 1.016 & 1.115 & 0.660 & 1 Mascot \\
\hline 1760.6517 & 1760.6782 & 0.0265 & 15 & 106 & 117 ETYGDMADCCEK & 53 & 99.845 & 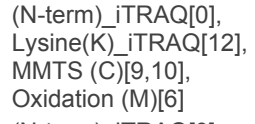 & , ${ }^{[2] ~ F 4 ~ a n d ~ F 13 ~}$ & 186/178 & 0.721 & 0.962 & 0.833 & 1 Mascot \\
\hline 1760.6517 & 1760.6796 & 0.0279 & 16 & 106 & 117 ETYGDMADCCEK & 80 & 100 & 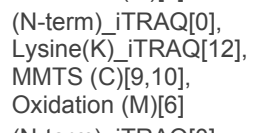 & , ${ }^{[4]}$ F11 and F3 & 1030/1022 & 0.965 & 0.949 & 0.867 & 1 Mascot \\
\hline 1829.8429 & 1829.7992 & -0.0437 & -24 & 387 & 399 EDPHACYATVFDK & 44 & 98.962 & $\begin{array}{l}\text { (N-term) iTRAQ[0]] } \\
\text { Lysine(K) iTRAQ[13], } \\
\text { MMTS (C)[6] }\end{array}$ & [4] F11 and F3 & $278 / 270$ & 1.101 & 0.923 & 1.044 & 1 Mascot \\
\hline 1829.8429 & 1829.8104 & -0.0325 & -18 & 387 & 399 EDPHACYATVFDK & 70 & 99.997 & 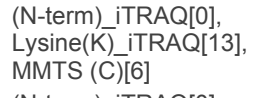 & [3] F $14,15,16$ and $F 6$ & 200/192 & 1.058 & 0.737 & 0.680 & 1 Mascot \\
\hline 1829.8429 & 1829.8975 & 0.0546 & 30 & 387 & 399 EDPHACYATVFDK & 98 & 100 & 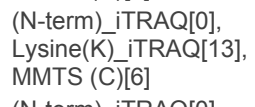 & [3] F14, 15,16 and F6 & $879 / 871$ & 1.304 & 1.103 & 0.679 & 1 Mascot \\
\hline 1829.8429 & 1829.9292 & 0.0863 & 47 & 387 & 399 EDPHACYATVFDK & 64 & 99.988 & 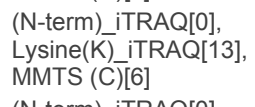 & $\begin{array}{l}\text { [7] F1 } 12 \text { and } F 9 \text { and F7 } \\
\text { attempt } 2\end{array}$ & $975 / 967$ & 1.498 & 1.988 & 0.928 & 1 Mascot \\
\hline 1857.9014 & 1857.882 & -0.0194 & -10 & 469 & 482 MPCTEDYLSLILNR & 76 & 99.999 & $\begin{array}{l}\text { (N-term) iTRAQ[0], } \\
\text { MMTS (C) }[3]\end{array}$ & {$[8] \mathrm{F} 2$} & $558 / 550$ & 0.855 & 0.856 & 0.879 & 1 Mascot \\
\hline 1860.6581 & 1860.5664 & -0.0917 & -49 & 267 & 280 ECCHGDLLECADDR & 92 & 100 & 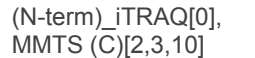 & [5] F5 and F10 & $474 / 466$ & 1.263 & 1.107 & 0.804 & 1 Mascot \\
\hline 1860.6581 & 1860.6014 & -0.0567 & -30 & 267 & 280 ECCHGDLLECADDR & 52 & 99.839 & $\begin{array}{l}\text { (N-erm) iTRAQ[0], } \\
\text { MMTS (C) }[2,3,10]\end{array}$ & {$[3] \mathrm{F} 14,15,16$ and F6 } & $221 / 213$ & 0.796 & 1.173 & 0.796 & 1 Mascot \\
\hline 1860.6581 & 1860.6028 & -0.0553 & -30 & 267 & 280 ECCHGDLLECADDR & 81 & 100 & $\begin{array}{l}\text { (N-term) iTRACI], } \\
\text { MMTS }(\bar{C})[2,3,10]\end{array}$ & [5] F5 and F10 & $407 / 399$ & 1.372 & 1.168 & 1.163 & 1 Mascot \\
\hline 1860.6581 & 1860.6277 & -0.0304 & -16 & 267 & 280 ECCHGDLLECADDR & 55 & 99.916 & $\begin{array}{l}\text { (N-term) } \text { (iTRAQ[0], } \\
\text { MMTS (C) }[2,3,10]\end{array}$ & [4] F11 and F3 & $329 / 321$ & 3.021 & 1.621 & 1.794 & 1 Mascot \\
\hline 1860.6581 & 1860.6558 & -0.0023 & -1 & 267 & 280 ECCHGDLLECADDR & 53 & 99.854 & $\begin{array}{l}\text { (N-term) iTRAC[0], } \\
\text { MMTS }(\bar{C})[2,3,10]\end{array}$ & [1] F8 061011 & 400/392 & 1.452 & 1.453 & 1.660 & 1 Mascot \\
\hline 1860.6581 & 1860.7362 & 0.0781 & 42 & 267 & 280 ECCHGDLLECADDR & 44 & 98.798 & 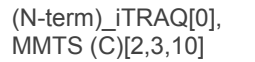 & [5] F5 and F10 & 1264/1256 & 1.608 & 2.083 & 1.057 & 1 Mascot \\
\hline 1860.6581 & 1860.74 & 0.0819 & 44 & 267 & 280 ECCHGDLLECADDR & 79 & 100 & 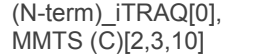 & [3] F14,15,16 and F6 & 930/922 & 1.021 & 1.161 & 1.116 & 1 Mascot \\
\hline 1866.1184 & 1866.0886 & -0.0298 & -16 & 402 & 413 HLVDEPQNLIKK & 91 & 100 & $\begin{array}{l}\text { (N-term) iTRAQ[0], } \\
\text { Lysine(K)_iTRAQ[11, } \\
\text { 12] }\end{array}$ & [5] F5 and F10 & $1151 / 1143$ & 1.080 & 1.402 & 0.894 & 1 Mascot \\
\hline 1873.8964 & 1873.8337 & -0.0627 & -33 & 469 & 482 MPCTEDYLSLILNR & 48 & 99.537 & $\begin{array}{l}\text { (N-term)iTRAQ[0], } \\
\text { MMTS (C)|3], } \\
\text { Oxidation (M)[1] }\end{array}$ & {$[8] \mathrm{F} 2$} & $525 / 517$ & 0.752 & 0.937 & 0.819 & 1 Mascot \\
\hline 1914.127 & 1914.0345 & -0.0925 & -48 & 437 & 451 KAPQVSTPTLVEISR & 58 & 99.954 & $\begin{array}{l}\text { (N-term) iTRAQ[0], } \\
\text { Lysine(Ki) iTAQA[1] }\end{array}$ & [5] F5 and F10 & $316 / 308$ & 1.282 & 1.017 & 1.003 & 1 Mascot \\
\hline 1914.127 & 1914.0424 & -0.0846 & -44 & 437 & 451 KAPQVSTPTLVEISR & 48 & 99.503 & 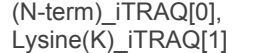 & [4] F11 and F3 & 239/231 & 0.866 & 0.990 & 0.889 & 1 Mascot \\
\hline 1914.127 & 1914.0715 & -0.0555 & -29 & 437 & 451 KAPQVSTPTLVEISR & 71 & 99.998 & $\begin{array}{l}\text { (N-term) iTRAQ[0], } \\
\text { Lysisen(K) }\end{array}$ & {$[3] \mathrm{F} 14,15,16$ and F6 } & $184 / 176$ & 0.831 & 0.937 & 0.889 & 1 Mascot \\
\hline 1914.127 & 1914.2155 & 0.0885 & 46 & 437 & 451 KAPQVSTPTLVEISR & 72 & 99.998 & 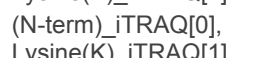 & {$[3] \mathrm{F} 14,15,16$ and F6 } & $833 / 825$ & 1.073 & 0.930 & 1.216 & 1 Mascot \\
\hline 1969.0491 & 1968.9862 & -0.0629 & -32 & 139 & 151 LKPEPDTLCAEFK & 57 & 99.945 & $\begin{array}{l}\text { (N-term) iTRAQ[0], } \\
\text { Lysine(K)) TRRAQ[2, } \\
\text { 3], MMTS (C)[9] }\end{array}$ & {$[3] \mathrm{F} 14,15,16$ and F6 } & $230 / 222$ & 1.025 & 1.108 & 0.958 & 1 Mascot \\
\hline 1969.0491 & 1968.9873 & -0.0618 & -31 & 139 & 151 LKPEPDTLCAEFK & 53 & 99.843 & 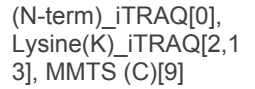 & [4] F11 and F3 & 347/339 & 0.799 & 0.931 & 0.875 & 1 Mascot \\
\hline 1969.0491 & 1969.0894 & 0.0403 & 20 & 139 & 151 LKPEPDTLCAEFK & 46 & 99.336 & $\begin{array}{l}\text { (N-term) iTRAQ[0], } \\
\text { Lysine(K)_iTAQQ[2,1 }\end{array}$ & [4] F11 and F3 & 959/951 & 0.966 & 0.935 & 1.158 & 1 Mascot \\
\hline
\end{tabular}




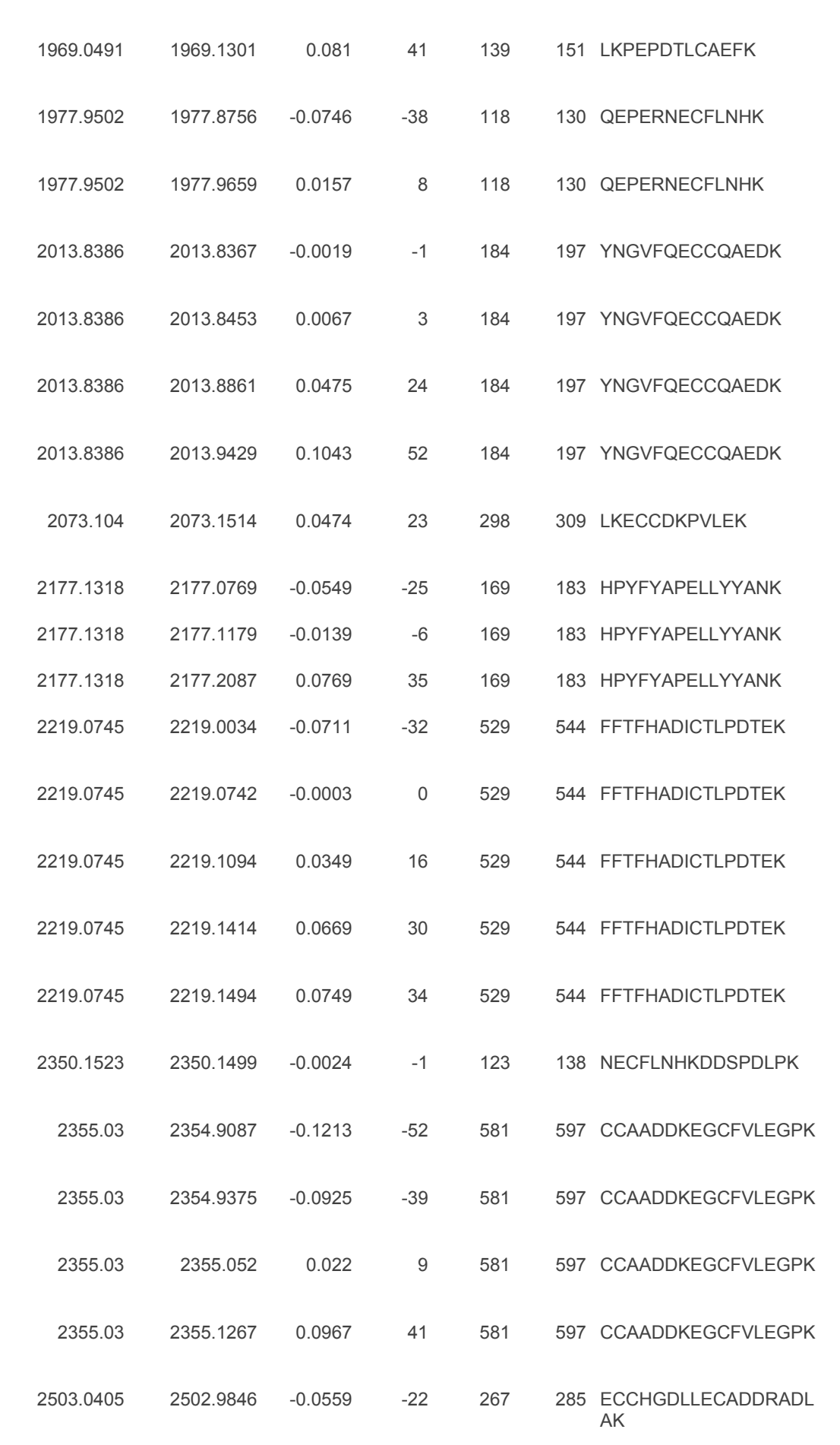

2 RecName: Full=Serotransferrin: Short=Transferrin:- gil2501351 888169
699997 3], MMTS (C) [9]

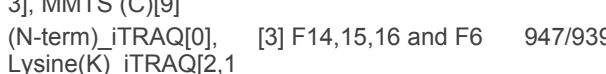
Lysine(K) - iTRAQ $[2$,
3 3], MMTS (C) $)(9]$

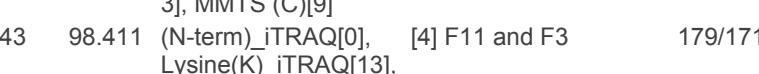
Lysine(K) ITRAQ[13],
MMTS (C) [8]

$46 \quad 99.336$ (N-term)iTRAQ[0], [5] F5 and F10 1132/1124 Lysine(K) iTRAQ[13,
MMTS (C) [8]

$70 \quad 99.997$ (N-term) iTRAQ[0], $[4] \mathrm{F} 11$ and F3 $\quad 1229 / 1221$

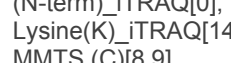

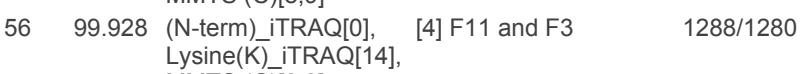

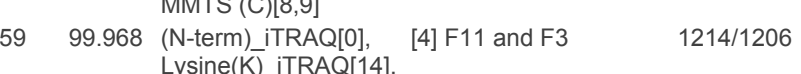

MMTS (C) [8.9]

$\left.74 \quad 99.999 \begin{array}{l}\text { (N-term) iTRAQ[0], } \\ \text { Lysine(K) }\end{array}\right]$ [4] F11 and F3 $1257 / 1249$ 99. MMTS (C) [8,9]

1226/1218

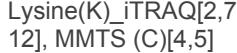

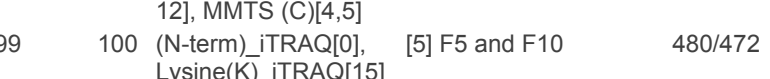

100 (N-term) iTRAQ[0], [5] F5 and F10 1349/1341

97.214 (N-term) iTRAQ[O], [3] F14,15,16 and F6 1019/1011

100 (N-term) ITRAQ[T], [5] F5 and F10 514/506

Lysine(K) iTRAQ[1
MMTS (C) [I]

100 (N-term) iTRAQ[0], [3] F14,15, 16 and F6 262/254

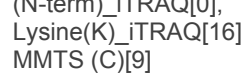

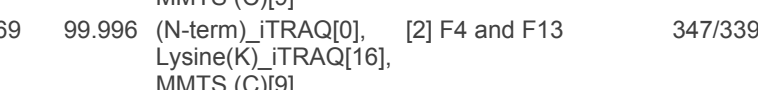

$49 \quad 99.648$ (N-term)iTRAQ[0], [2] F4 and F13 $\quad 438 / 430$

$$
\begin{aligned}
& 8 \text { (N-term)iTRAQ[0], } \\
& \text { Lysine(K)iTRAQ[16], } \\
& \text { MMTS (C)[9] }
\end{aligned}
$$

$46 \quad 99.305$ (N-term) iTRAQ[0], [2] F4 and F13 1030/1022

Lysine(K) iTRAQ[16],

$47 \quad 99.381 \quad($ N-term) iTRAQ[0], $\quad[5] \mathrm{F} 5$ and $\mathrm{F} 10 \quad 1194 / 1186$

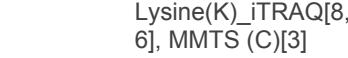

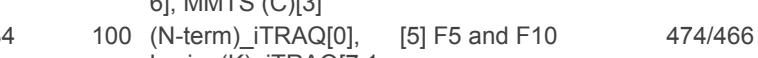

Lysine(K) ITRAQ[7,1
7], MMTS (C) $)(1,2,10]$

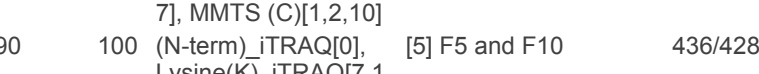

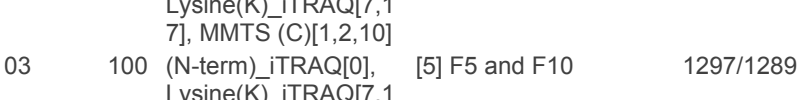

99.817 7], MMTS (C)[1,2, 10]

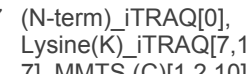

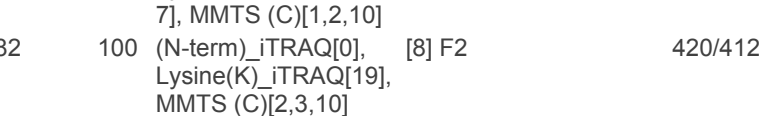

$0.951-0.901-0.884=0.438$

\begin{tabular}{llll}
1.047 & 0.910 & 0.908 & 1 Mascot \\
0.880 & 0.897 & 1.109 & 1 Mascot \\
1.049 & 1.105 & 1.025 & 1 Mascot \\
2.242 & 2.031 & 0.895 & 1 Mascot \\
2.093 & 1.654 & 0.932 & 1 Mascot \\
1.826 & 1.527 & 1.043 & 1 Mascot \\
1.930 & 1.969 & 0.998 & 1 Mascot \\
1.084 & 1.054 & 0.971 & 1 Mascot \\
0.566 & 0.661 & 0.736 & 1 Mascot \\
0.465 & 0.762 & 0.976 & 1 Mascot \\
0.811 & 0.811 & 1.058 & 1 Mascot \\
0.705 & 1.027 & 0.870 & 1 Mascot \\
0.844 & 1.077 & 0.823 & 1 Mascot \\
\hline 0.856 & 1.004 & 1.010 & 1 Mascot \\
1.072 & 1.012 & 1.062 & 1 Mascot \\
\hline 0.987 & 1.128 & 0.653 & 1 Mascot \\
0.690 & 0.621 & 0.812 & 1 Mascot \\
2.389 & 2.697 & 1.331 & 1 Mascot \\
2.489 & 2.259 & 1.224 & 1 Mascot \\
3.319 & 3.761 & 1.524 & 1 Mascot \\
1.476 & 2.779 & 1.087 & 1 Mascot \\
1.456 & 1.205 & 0.971 & 1 Mascot \\
\hline & & & \\
\hline
\end{tabular}

100

\begin{tabular}{|c|c|c|c|c|c|c|c|c|c|c|c|c|c|c|}
\hline \multicolumn{2}{|c|}{ Calc. Mass Obsrv. Mass } & \multicolumn{2}{|c|}{ \pm da \pm ppm } & $\begin{array}{l}\text { Start } \\
\text { Seq. }\end{array}$ & $\begin{array}{l}\text { End Sequence } \\
\text { Seq. }\end{array}$ & $\begin{array}{l}\text { lon } \\
\text { Score }\end{array}$ & \multicolumn{2}{|c|}{ C. I. \% Modification } & Plate [\#] Name & $\begin{array}{l}\text { Gel Idx/Pos [4700 } \\
\text { Sample Name] }\end{array}$ & $\begin{array}{r}\text { iTRAQ } \\
\text { Ratio } \\
114 / 117^{*}\end{array}$ & $\begin{array}{r}\text { iTRAQ } \\
\text { Ratio } \\
115 / 117^{*}\end{array}$ & $\begin{array}{r}\text { iTRAQ } \\
\text { Ratio } \\
116 / 117^{*}\end{array}$ & Rank Result Type \\
\hline 10236084 & 10235566 & -0.0518 & -51 & 554 & 560 GDVAFVK & 42 & 98313 & & 15] E5 and F10 & 2081200 & 0800 & 0707 & 0728 & 1 Macset \\
\hline & & & & & & & & Lysine(K)_iTRAQ[7] & & & & & & \\
\hline 1140.6146 & 1140.5513 & -0.0633 & -55 & 320 & 327 DSADGFLK & 49 & 99.679 & (N-term) iTRAQ[0], & [5] F5 and F10 & $248 / 240$ & 0.845 & 0.890 & 1.037 & 1 Mascot \\
\hline 1140.6146 & 1140.6289 & 0.0143 & 13 & 320 & 327 DSADGFLK & 40 & 96.994 & 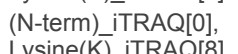 & [5] F5 and F10 & 1119/1111 & 0.394 & 0.629 & 0.385 & 1 Mascot \\
\hline 1207.766 & 1207.7739 & 0.0079 & 7 & 448 & 456 GYLAVAVVK & 61 & 99.976 & (N-term) iTRAQ[0], & [4] F11 and F3 & 1081/1073 & 0.761 & 0.721 & 0.777 & 1 Mascot \\
\hline 1215.593 & 1215.6121 & 0.0191 & 16 & 188 & 195 LCQLCAGK & 58 & 99.95 & 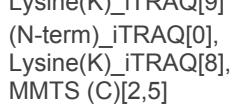 & [2] F4 and F13 & $267 / 259$ & 2.625 & 2.161 & 1.404 & 1 Mascot \\
\hline 1241.6075 & 1241.6476 & 0.0401 & 32 & 540 & 548 YYGYTGAFR & 38 & 95.945 & (N-term) iTRAQ[0] & [4] F11 and F3 & 1063/1055 & 1.179 & 1.029 & 1.001 & 1 Mascot \\
\hline 1268.6028 & 1268.5626 & -0.0402 & -32 & 61 & 68 TSHMDCIK & 55 & 99.914 & 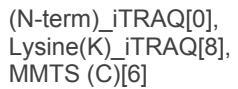 & [1] F8 061011 & $220 / 212$ & 0.912 & 0.857 & 0.783 & 1 Mascot \\
\hline
\end{tabular}
(N-term) iTRAQ
Lysine(K) iTRAQ
MMTS (C)[6] 


\begin{tabular}{|c|c|c|c|c|c|}
\hline 1455.7563 & 1455.6851 & -0.0712 & -49 & 156 & 166 ELPDPQESIQR \\
\hline 1455.7563 & 1455.7013 & -0.055 & -38 & 156 & 166 ELPDPQESIQR \\
\hline 1455.7563 & 1455.8163 & 0.06 & 41 & 156 & 166 ELPDPQESIQR \\
\hline 1492.7883 & 1492.7474 & -0.0409 & -27 & 695 & 704 LLEACTFHKP \\
\hline 1492.7883 & 1492.7501 & -0.0382 & -26 & 695 & 704 LLEACTFHKP \\
\hline 1492.7883 & 1492.8335 & 0.0452 & 30 & 695 & 704 LLEACTFHKP \\
\hline 1556.7952 & 1556.6943 & -0.1009 & -65 & 60 & $68 \mathrm{KTSHMDCIK}$ \\
\hline 1610.7458 & 1610.7025 & -0.0433 & -27 & 671 & 682 TYDSYLGDDYVR \\
\hline 1610.7458 & 1610.7777 & 0.0319 & 20 & 671 & 682 TYDSYLGDDYVR \\
\hline 1610.7458 & 1610.7975 & 0.0517 & 32 & 671 & 682 TYDSYLGDDYVR \\
\hline 1623.785 & 1623.8906 & 0.1056 & 65 & 27 & 37 WCTISTHEANK \\
\hline 1639.8778 & 1639.9443 & 0.0665 & 41 & 424 & 435 CGLVPVLAENYK \\
\hline 1839.0084 & 1839.0402 & 0.0318 & 17 & 480 & 493 TAGWNIPMGLLYSK \\
\hline 1882.9432 & 1882.8854 & -0.0578 & -31 & 670 & 682 KTYDSYLGDDYVR \\
\hline 1882.9432 & 1883.0215 & 0.0783 & 42 & 670 & 682 KTYDSYLGDDYVR \\
\hline 1893.0116 & 1892.9187 & -0.0929 & -49 & 107 & 120 DNPQTHYYAVAVVK \\
\hline 1893.0116 & 1892.9353 & -0.0763 & -40 & 107 & 120 DNPQTHYYAVAVVK \\
\hline 1893.0116 & 1892.9382 & -0.0734 & -39 & 107 & 120 DNPQTHYYAVAVVK \\
\hline 1893.0116 & 1892.9613 & -0.0503 & -27 & 107 & 120 DNPQTHYYAVAVVK \\
\hline 1893.0116 & 1892.9622 & -0.0494 & -26 & 107 & 120 DNPQTHYYAVAVVK \\
\hline 1893.0116 & 1893.058 & 0.0464 & 25 & 107 & 120 DNPQTHYYAVAVVK \\
\hline 1893.0116 & 1893.074 & 0.0624 & 33 & 107 & 120 DNPQTHYYAVAVVK \\
\hline 2004.1012 & 2004.0406 & -0.0606 & -30 & 153 & 166 LYKELPDPQESIQR \\
\hline 2004.1012 & 2004.1591 & 0.0579 & 29 & 153 & 166 LYKELPDPQESIQR \\
\hline 2119.0627 & 2118.9917 & -0.071 & -34 & 406 & 423 GEADAMSLDGGYLYIAG \\
\hline 2119.0627 & 2119.0623 & -0.0004 & 0 & 406 & 423 GEADAMSLDGGYLYIAG \\
\hline 2190.168 & 2190.1929 & 0.0249 & 11 & 300 & $\begin{array}{l}\text { 314 DKPDNFQLFQSPHGK } \\
314\end{array}$ \\
\hline 2353.2188 & 2353.2102 & -0.0086 & -4 & 283 & 299 EDVIWELLNHAQEHFGK \\
\hline 2635.3845 & 2635.2712 & -0.1133 & -43 & 403 & 423 IMKGEADAMSLDGGYLYI \\
\hline
\end{tabular}
RecName: Full=Serum albumin; AltName: Full=BSA; $\quad$ gil1351907
AltName; Allergen=Bos $d 6$; Flags: Precursor
$39 \quad 96.643$ (N-term)_iTRAQ[0] $\quad[5]$ F5 and F10 $256 / 248$

$\begin{array}{llll}40 & 96.83 \text { (N-term)_iTRAQ[0] } & {[8] \mathrm{F} 2} & 265 / 257\end{array}$

$46 \quad 99.277$ (N-term)_iTRAQ[0] [4] F11 and F3 1006/998

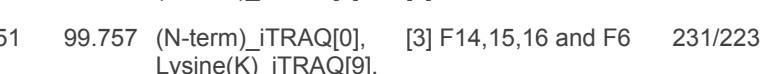
MMTS (C)[5] $[5]$

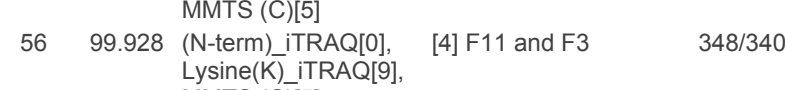

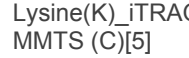

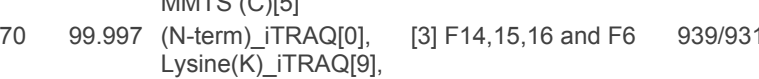

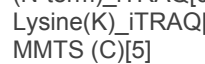

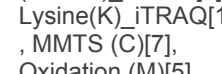

$89 \begin{array}{lll}\text { Oxidation }(\mathrm{M})[5] \\ \text { (N-term)_TRAQ[0] }\end{array}$

6799.994 (N-term)_iTRAQ[0] [4] F11 and F3 $\quad$ 1076/1068

4398.493 (N-term)_iTRAQ[0] [3] F14,15,16 and F6 $853 / 845$

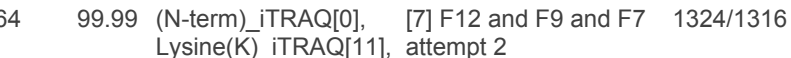
Lysine(K) iTRAQ[11], attempt 2
MMTS (C)[2]

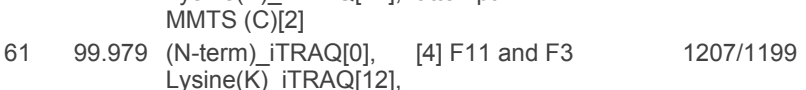

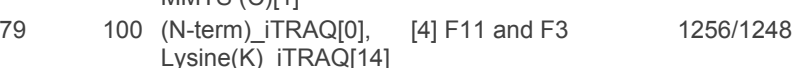

5599.91 (N-term) iTRAC[0], [8] F2 $311 / 303$

$40 \quad 97.156$ (N-term) iTRAQ[0], [7] F12 and F9 and F7 1302/1294

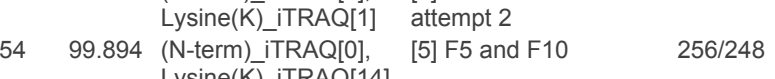

98100 (N-term)_iTRAQ[0], [5] F5 and F10 306/298

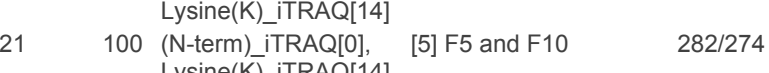

$40 \quad 96.816$ (N-term) iTRAQ[0], [5] F5 and F10 303/295

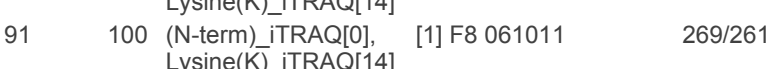

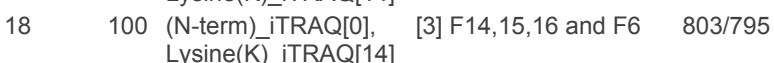

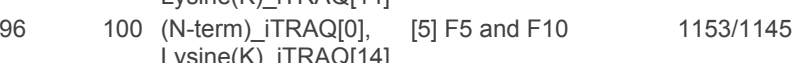

$58 \quad 99.951$ (N-term) iTRAQ[0], [5] F5 and F10 326/318

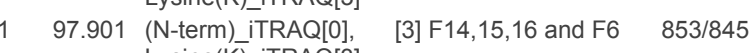

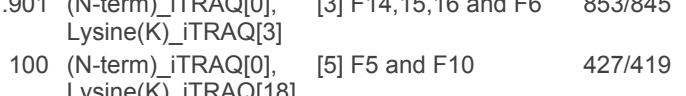

100 (N-term) iTRAQ[0] [4] F11 and F3 1175/1167

Lysine(K) iTRAQ[18]
(N-term) iTRAQ[0], [5] F5 and F10 1176/1168

(5)

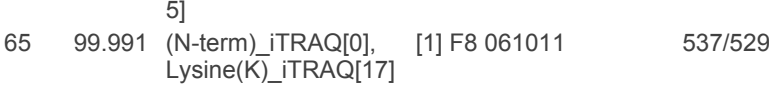

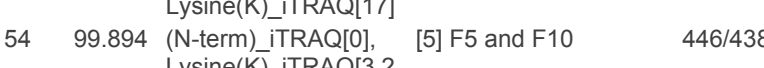

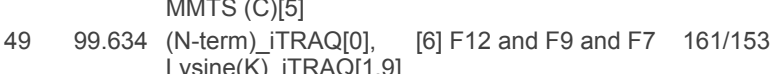

\begin{tabular}{|c|c|c|c|}
\hline 0.895 & 0.941 & 0.940 & 1 Mascot \\
\hline 0.997 & 0.872 & 0.859 & 1 Mascot \\
\hline 0.869 & 0.816 & 0.942 & 1 Mascot \\
\hline 1.564 & 1.614 & 1.149 & 1 Mascot \\
\hline 1.019 & 1.067 & 1.175 & 1 Mascot \\
\hline 1.273 & 1.133 & 1.035 & 1 Mascot \\
\hline 1.208 & 1.128 & 1.012 & 1 Mascot \\
\hline 1.288 & 1.129 & 1.053 & 1 Mascot \\
\hline 1.262 & 1.063 & 1.189 & 1 Mascot \\
\hline 1.543 & 1.609 & 1.498 & 1 Mascot \\
\hline 0.933 & 0.861 & 0.906 & 1 Mascot \\
\hline 2.141 & 1.164 & 0.439 & 1 Mascot \\
\hline 1.561 & 1.633 & 1.284 & 1 Mascot \\
\hline 0.918 & 0.787 & 1.095 & 1 Mascot \\
\hline 0.932 & 0.881 & 0.971 & 1 Mascot \\
\hline 0.870 & 0.549 & 0.791 & 1 Mascot \\
\hline 0.696 & 0.632 & 1.051 & 1 Mascot \\
\hline 0.740 & 0.686 & 0.761 & 1 Mascot \\
\hline 0.695 & 0.390 & 0.666 & 1 Mascot \\
\hline 1.034 & 1.017 & 0.774 & 1 Mascot \\
\hline 0.459 & 0.696 & 0.905 & 1 Mascot \\
\hline 0.746 & 0.829 & 1.022 & 1 Mascot \\
\hline 1.212 & 1.390 & 1.210 & 1 Mascot \\
\hline 0.903 & 1.258 & 0.855 & 1 Mascot \\
\hline 0.805 & 0.891 & 0.843 & 1 Mascot \\
\hline 0.625 & 0.597 & 0.749 & 1 Mascot \\
\hline 0.560 & 0.619 & 0.817 & 1 Mascot \\
\hline 0.700 & 0.746 & 0.854 & 1 Mascot \\
\hline 0.926 & 0.583 & 0.316 & \\
\hline
\end{tabular}

$\begin{array}{llllll}18 & 1217 & 0.953 & 1.101 & 0.949 & 0.568\end{array}$

Mascot ascot

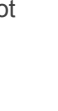

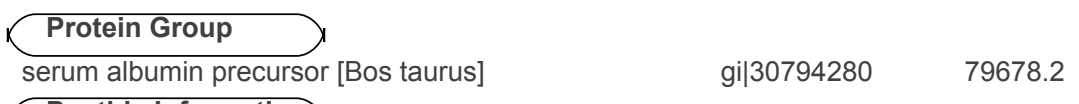




$\begin{array}{lccccl}1175.6508 & 1175.6975 & 0.0467 & 40 & 483 & 489 \text { LCVLHEK } \\ 1260.5291 & 1260.5676 & 0.0385 & 31 & 499 & 507 \text { CCTESLVNR } \\ 1302.8242 & 1302.7657 & -0.0585 & -45 & 549 & 557 \text { QTALVELLK } \\ 1302.8242 & 1302.7715 & -0.0527 & -40 & 549 & 557 \text { QTALVELLK } \\ 1302.8242 & 1302.8359 & 0.0117 & 9 & 549 & 557 \text { QTALVELLK } \\ 1302.8242 & 1302.8368 & 0.0126 & 10 & 549 & 557 \text { QTALVELLK } \\ 1302.8242 & 1302.8618 & 0.0376 & 29 & 549 & 557 \text { QTALVELLK } \\ 1427.8131 & 1427.851 & 0.0379 & 27 & 361 & 371 \text { HPEYAVSVLLR } \\ 1575.0216 & 1574.9834 & -0.0382 & -24 & 548 & 557 \text { KQTALVELLK } \\ 1575.0216 & 1575.0242 & 0.0026 & 2 & 548 & 557 \text { KQTALVELLK } \\ 1575.0216 & 1575.1365 & 0.1149 & 73 & 548 & 557 \text { KQTALVELLK } \\ 1593.921 & 1593.8564 & -0.0646 & -41 & 402 & 412 \text { HLVDEPQNLIK } \\ 1593.921 & 1593.8634 & -0.0576 & -36 & 402 & 412 \text { HLVDEPQNLIK } \\ 1593.921 & 1593.975 & 0.054 & 34 & 402 & 412 \text { HLVDEPQNLIK } \\ 1593.921 & 1593.9929 & 0.0719 & 45 & 402 & 412 \text { HLVDEPQNLIK } \\ 1593.921 & 1594.0195 & 0.0985 & 62 & 402 & 412 \text { HLVDEPQNLIK } \\ 1687.8975 & 1687.8418 & -0.0557 & -33 & 569 & 580 \text { TVMENFVAFVDK } \\ 1687.8975 & 1687.8925 & -0.005 & -3 & 569 & 580 \text { TVMENFVAFVDK } \\ 1687.8975 & 1687.9059 & 0.0084 & 5 & 569 & 580 \text { TVMENFVAFVDK } \\ 1687.8975 & 1677.9149 & 0.0174 & 10 & 569 & 580 \text { TVMENFVAFVDK } \\ 1687.8975 & 1687.9182 & 0.0207 & 12 & 569 & 580 \text { TVMENFVAFVDK } \\ 1687.8975 & 1687.9308 & 0.0333 & 20 & 569 & 580 \text { TVMENFVAFVDK } \\ 1687.8975 & 1687.9449 & 0.0474 & 28 & 569 & 580 \text { TVMENFVAFVDK } \\ 1687.8975 & 1687.9557 & 0.0582 & 34 & 569 & 580 \text { TVMENFVAFVDK } \\ 1687.8975 & 1687.9612 & 0.0637 & 38 & 569 & 580 \text { TVMENFVAFVDK } \\ 1696.8629 & 1696.818 & -0.0449 & -26 & 89 & 100 \text { SLHTLFGDELCK } \\ 16996.8629 & 1696.8333 & -0.0296 & -17 & 89 & 100 \text { SLHTLFGDELCK } \\ 1696.8629 & 1696.9674 & 0.1045 & 62 & 89 & 100 \text { SLHTLFGDELCK }\end{array}$

$40 \quad$ MMTS (C)[2]

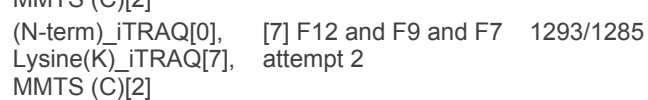

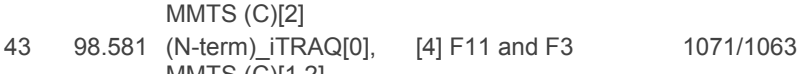

$44 \quad 98.958$ (N-term) iTRAQ[0], $[3] \mathrm{F} 14,15,16$ and F6 $\quad 226 / 218$

$\left.64 \quad 99.99 \begin{array}{c}\text { Lysine(K)_iTRAQ[9] } \\ \text { (N-term) iTRAQ[0], } \\ \text { Lysine(K)_TRAQ[9] }\end{array}\right]$ [5] F5 and F10 $409 / 401$

$70 \quad 99.997$ (N-term)_iTRAQ[0], [4] F11 and F3 $\quad 1149 / 1141$

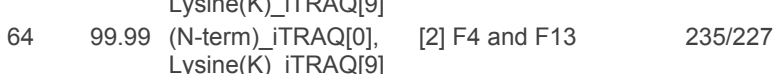

6499.988 (N-term) iTRAQ[0], [2] F4 and F13 995/987

$41 \quad 97.693$ (N-term) iTRAQ[0] [3] F14,15,16 and F6 $902 / 894$

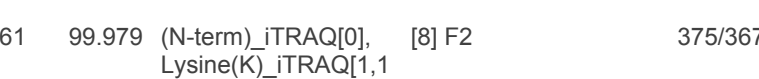

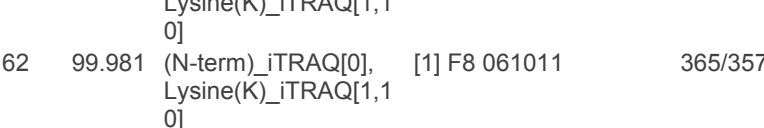

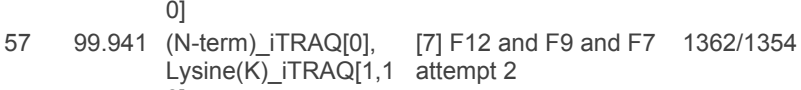
Lysine(K)_iTRAQ[1 1 attempt 2
$0]$
(N-term)_iTRAQ[0], [3] F14,15,16 and F6 $\quad 174 / 166$

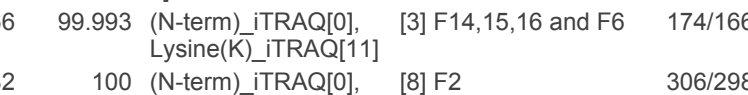

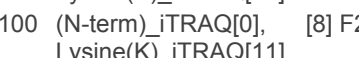

$306 / 298$
$821 / 813$

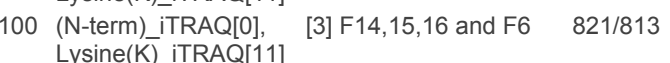

4499.879 (N-term) iTRAQ[0], [7] F12 and F9 and F7 1293/1285

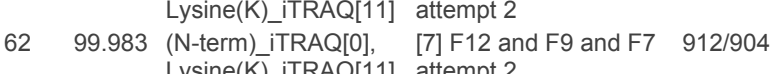

$100 \quad 100($ N-term) iTRAQ[0], [5] F5 and F10 512/504

$6799.994 \begin{aligned} & \text { (N-term) iTRAQ[0], } \\ & \text { Lysine (K)] }\end{aligned}$

$47 \quad 99.392$ (N-term) iTRAQ[0], [4] F11 and F3 1231/1223

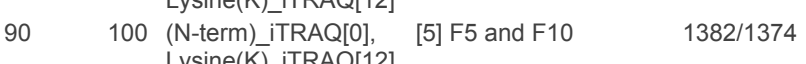

102100 (N-term)_iTRAQ[0], [2] F4 and F13 348/340

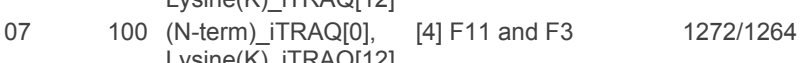

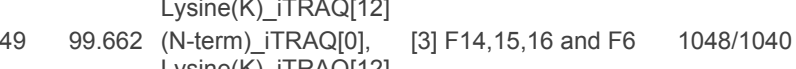

$46 \quad 99.272$ (N-term) iTRAQ[0], [2] F4 and F13 1031/1023

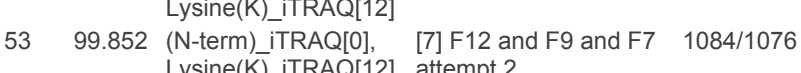

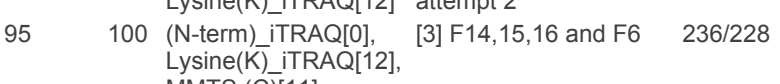

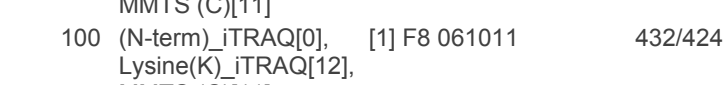

$\left.100 \begin{array}{c}\text { (N-term) iTRAC[0], } \\ \text { Lysine(K) iTRA[12], }\end{array}\right]$ [4] F11 and F3 $360 / 352$

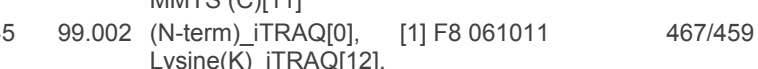

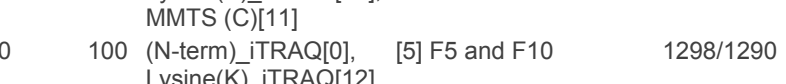

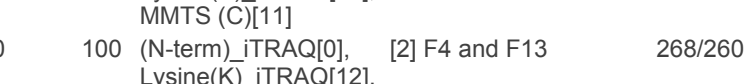
MMTS (C) [11] 11$]$

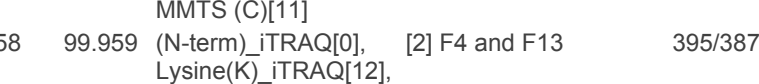
Lysine(K) iTRAQL 121
MMTS (C) $[11]$

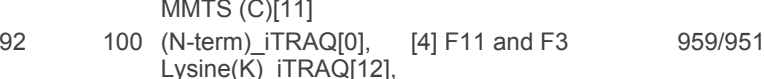
Lysine(K) ${ }_{\text {iTRAQQ12 }}$
MMTS (C) $[11]$

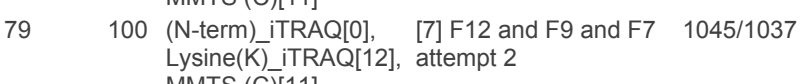

95100 (N-term) iTRAQ[0], [3] F14,15, 16 and F6 963/955

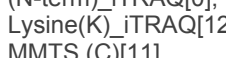

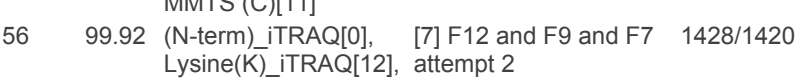

\begin{tabular}{|c|c|c|c|}
\hline 1.301 & 1.047 & 0.748 & 1 Mascot \\
\hline 1.992 & 2.408 & 1.293 & 1 Mascot \\
\hline 1.249 & 2.368 & 1.376 & 1 Mascot \\
\hline 1.135 & 1.403 & 0.860 & 1 Mascot \\
\hline 1.196 & 1.443 & 0.987 & 1 Mascot \\
\hline 0.663 & 1.379 & 1.131 & 1 Mascot \\
\hline 0.751 & 1.218 & 1.051 & 1 Mascot \\
\hline 0.682 & 0.665 & 0.694 & 1 Mascot \\
\hline 1.065 & 1.281 & 1.002 & 1 Mascot \\
\hline 0.570 & 1.183 & 1.125 & 1 Mascot \\
\hline 0.912 & 0.993 & 0.968 & 1 Mascot \\
\hline 1.536 & 1.441 & 0.941 & 1 Mascot \\
\hline 0.873 & 0.928 & 0.888 & 1 Mascot \\
\hline 1.128 & 0.994 & 0.937 & 1 Mascot \\
\hline 0.851 & 0.864 & 0.846 & 1 Mascot \\
\hline 1.204 & 1.723 & 1.246 & 1 Mascot \\
\hline 1.113 & 1.245 & 0.975 & 1 Mascot \\
\hline 0.506 & 1.255 & 0.753 & 1 Mascot \\
\hline 0.887 & 1.062 & 0.891 & 1 Mascot \\
\hline 1.453 & 1.560 & 0.727 & 1 Mascot \\
\hline 0.914 & 1.109 & 0.881 & 1 Mascot \\
\hline 1.120 & 1.121 & 0.952 & 1 Mascot \\
\hline 1.092 & 0.966 & 1.058 & 1 Mascot \\
\hline 1.939 & 1.118 & 2.089 & 1 Mascot \\
\hline 1.666 & 1.429 & 1.798 & 1 Mascot \\
\hline 0.515 & 0.534 & 0.670 & 1 Mascot \\
\hline 0.417 & 0.545 & 0.730 & 1 Mascot \\
\hline 0.533 & 0.549 & 0.628 & 1 Mascot \\
\hline 0.333 & 0.594 & 0.650 & 1 Mascot \\
\hline 1.501 & 2.002 & 1.278 & 1 Mascot \\
\hline 0.740 & 1.016 & 1.053 & 1 Mascot \\
\hline 1.702 & 1.957 & 2.371 & 1 Mascot \\
\hline 0.444 & 0.630 & 0.658 & 1 Mascot \\
\hline 1.127 & 1.204 & 1.089 & 1 Mascot \\
\hline 0.565 & 0.588 & 0.718 & 1 Mascot \\
\hline 0.293 & 0.582 & 0.761 & 1 Mascot \\
\hline
\end{tabular}




\begin{tabular}{|c|c|c|c|c|c|}
\hline 1703.8925 & 1703.8389 & -0.0536 & -31 & 569 & 580 TVMENFVAFVDK \\
\hline 1703.8925 & 1703.9235 & 0.031 & 18 & 569 & 580 TVMENFVAFVDK \\
\hline 1703.8925 & 1703.9277 & 0.0352 & 21 & 569 & 580 TVMENFVAFVDK \\
\hline 1729.7225 & 1729.6005 & -0.122 & -71 & 76 & 88 TCVADESHAGCEK \\
\hline 1744.6569 & 1744.5756 & -0.0813 & -47 & 106 & 117 ETYGDMADCCEK \\
\hline 1744.6569 & 1744.6769 & 0.02 & 11 & 106 & 117 ETYGDMADCCEK \\
\hline 1744.6569 & 1744.6855 & 0.0286 & 16 & 106 & 117 ETYGDMADCCEK \\
\hline 1744.6569 & 1744.6956 & 0.0387 & 22 & 106 & 117 ETYGDMADCCEK \\
\hline 1744.6569 & 1744.717 & 0.0601 & 34 & 106 & 117 ETYGDMADCCEK \\
\hline 1744.6569 & 1744.7797 & 0.1228 & 70 & 106 & 117 ETYGDMADCCEK \\
\hline 1760.6517 & 1760.6782 & 0.0265 & 15 & 106 & 117 ETYGDMADCCEK \\
\hline 1760.6517 & 1760.6796 & 0.0279 & 16 & 106 & 117 ETYGDMADCCEK \\
\hline 1857.9014 & 1857.882 & -0.0194 & -10 & 469 & 482 MPCTEDYLSLILNR \\
\hline 1860.6581 & 1860.5664 & -0.0917 & -49 & 267 & 280 ECCHGDLLECADDR \\
\hline 1860.6581 & 1860.6014 & -0.0567 & -30 & 267 & 280 ECCHGDLLECADDR \\
\hline 1860.6581 & 1860.6028 & -0.0553 & -30 & 267 & 280 ECCHGDLLECADDR \\
\hline 1860.6581 & 1860.6277 & -0.0304 & -16 & 267 & 280 ECCHGDLLECADDR \\
\hline 1860.6581 & 1860.6558 & -0.0023 & -1 & 267 & 280 ECCHGDLLECADDR \\
\hline 1860.6581 & 1860.7362 & 0.0781 & 42 & 267 & 280 ECCHGDLLECADDR \\
\hline 1860.6581 & 1860.74 & 0.0819 & 44 & 267 & 280 ECCHGDLLECADDR \\
\hline 1873.8964 & 1873.8337 & -0.0627 & -33 & 469 & 482 MPCTEDYLSLILNR \\
\hline 2013.8386 & 2013.8367 & -0.0019 & -1 & 184 & 197 YNGVFQECCQAEDK \\
\hline 2013.8386 & 2013.8453 & 0.0067 & 3 & 184 & 197 YNGVFQECCQAEDK \\
\hline 2013.8386 & 2013.8861 & 0.0475 & 24 & 184 & 197 YNGVFQECCQAEDK \\
\hline 2013.8386 & 2013.9429 & 0.1043 & 52 & 184 & 197 YNGVFQECCQAEDK \\
\hline 2177.1318 & 2177.0769 & -0.0549 & -25 & 169 & 183 HPYFYAPELLYYANK \\
\hline 2177.1318 & 2177.1179 & -0.0139 & -6 & 169 & 183 HPYFYAPELLYYANK \\
\hline 2177.1318 & 2177.2087 & 0.0769 & 35 & 169 & 183 HPYFYAPELLYYANK \\
\hline 2503.0405 & 2502.9846 & -0.0559 & -22 & 267 & 285 ECCHGDLLECADDRADL \\
\hline
\end{tabular}

4 RecName: Full=Angiotensinogen; AltName: Full=Serpin gil1703309

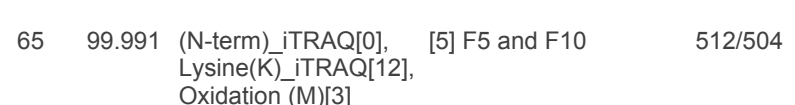

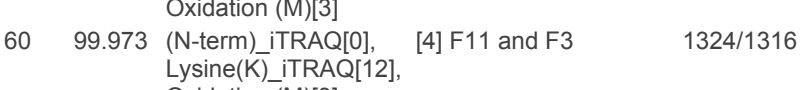

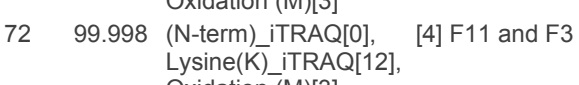

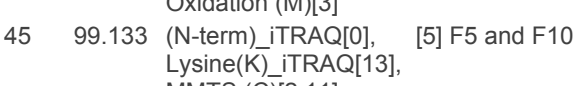
$81 \quad 100 \begin{aligned} & \text { (N-term) }[\text { iTRAR[0], } \\ & \text { Lysine(K) iTRAQ[12], }\end{aligned},[5]$ F5 and F10

$85100 \begin{gathered}\text { MN-term) IiTRAA[0], } \\ \text { LLsine(K) iTRAQ[12], }\end{gathered}$ [2] F4 and F13 MMTS (C) $[9,10]$

50 99.723 (N-term) _TRAQ[0], [4] F11 and F3

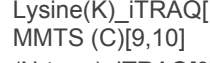

$100($ N-term) $i$ TRAQ[0], $\quad[4] \mathrm{F} 11$ and F 3 Lysine $(K)$ iTRAQ $[12]$
MMTS (C) $[1,10]$

6599.991 (N-term) itRAQ[0], [2] F4 and F13 Lysine(K) iTRAQ[1,
MMTS (C) $[9,10]$

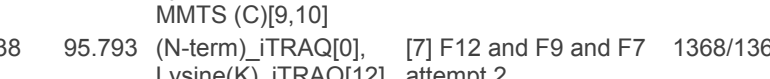
Lysine(K) ITRAQ[12], attempt 2

$53 \quad 99.845$ (N-term) iTRAQ[0], [2] F4 and F13 $186 / 178$

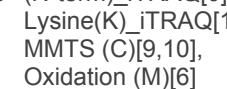

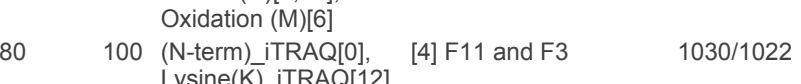
Lysine(K) iTRAA
MMTS(C)[9,10],
Oxidation (M) [ [ 6$]$

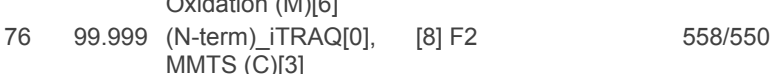

92100 (N-term) iTRAQ[0], [5] F5 and F10 $474 / 466$

92.839 (N-term) ITRAQ[0], $[3] \mathrm{F} 14,15,16$ and F6 $\quad 221 / 213$

100 (N-term) iTRAQ[0], [5] F5 and F10 $407 / 399$

99.916 (N-term) iTRAO[0] [4] F11 and F3 329/321

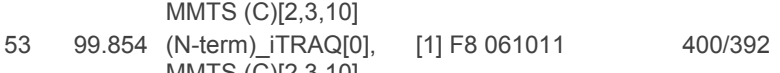

$44 \quad 98.798$ (N-term) iTRAQ[0], [5] F5 and F10 1264/1256

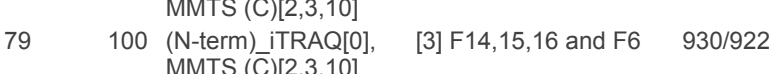

$48 \quad 99.537$ (N-ters)_iTRAQ[0], $\quad[8] \mathrm{F} 2$

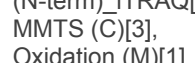

$70 \quad 99.997$ (N-term) iTRAQ[0], [4] F11 and F3 1229/1221 Lysine(K) $(T \mathrm{TRAQ}[14]$

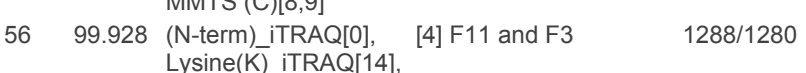

MMTS (C)[8,9]

$\left.99.968 \begin{array}{c}\text { N-term) iTRAQ[0], } \\ \text { Lysine(K) }(\text { iTRAQ[14] }\end{array}\right]$

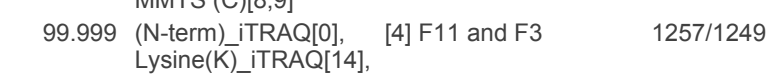

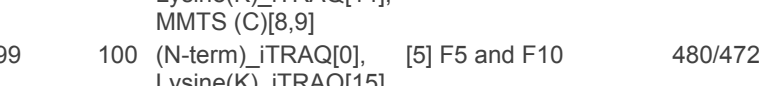

79 (N-term) ITRAQ[0], [5] F5 and F10 1349/1341

4097.214 (N-term) iTRAQ[0], [3] F14,15,16 and F6 1019/1011

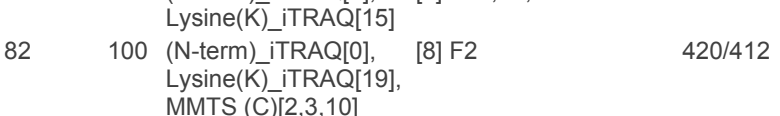

\begin{tabular}{|c|c|c|c|}
\hline 0.947 & 0.682 & 0.857 & 1 Mascot \\
\hline 0.981 & 1.153 & 0.881 & 1 Mascot \\
\hline 1.040 & 1.306 & 1.265 & 1 Mascot \\
\hline 0.627 & 1.483 & 0.380 & 1 Mascot \\
\hline 0.601 & 0.935 & 0.924 & 1 Mascot \\
\hline 0.564 & 1.022 & 0.953 & 1 Mascot \\
\hline 1.209 & 1.073 & 0.938 & 1 Mascot \\
\hline 0.855 & 0.800 & 0.763 & 1 Mascot \\
\hline 0.542 & 1.061 & 0.868 & 1 Mascot \\
\hline 1.180 & 1.412 & 0.907 & 1 Mascot \\
\hline 0.721 & 0.962 & 0.833 & 1 Mascot \\
\hline 0.965 & 0.949 & 0.867 & 1 Mascot \\
\hline 0.855 & 0.856 & 0.879 & 1 Mascot \\
\hline 1.263 & 1.107 & 0.804 & 1 Mascot \\
\hline 0.796 & 1.173 & 0.796 & 1 Mascot \\
\hline 1.372 & 1.168 & 1.163 & 1 Mascot \\
\hline 3.021 & 1.621 & 1.794 & 1 Mascot \\
\hline 1.452 & 1.453 & 1.660 & 1 Mascot \\
\hline 1.608 & 2.083 & 1.057 & 1 Mascot \\
\hline 1.021 & 1.161 & 1.116 & 1 Mascot \\
\hline 0.752 & 0.937 & 0.819 & 1 Mascot \\
\hline 2.242 & 2.031 & 0.895 & 1 Mascot \\
\hline 2.093 & 1.654 & 0.932 & 1 Mascot \\
\hline 1.826 & 1.527 & 1.043 & 1 Mascot \\
\hline 1.930 & 1.969 & 0.998 & 1 Mascot \\
\hline 0.566 & 0.661 & 0.736 & 1 Mascot \\
\hline 0.465 & 0.762 & 0.976 & 1 Mascot \\
\hline 0.811 & 0.811 & 1.058 & 1 Mascot \\
\hline 1.456 & 1.205 & 0.971 & 1 Mascot \\
\hline
\end{tabular}




$\begin{array}{rrrrrl}1188.6609 & 1188.7065 & 0.0456 & 38 & 459 & 468 \text { SSGALHFLGR } \\ 1449.7795 & 1449.7296 & -0.0499 & -34 & 82 & 91 \text { ATEKLEAEDR } \\ 1449.8927 & 1449.9207 & 0.028 & 19 & 415 & 424 \text { VLNSVLFELK } \\ 1584.957 & 1584.9668 & 0.0098 & 6 & 392 & 404 \text { LPTLLGAEANLGK } \\ 1622.0251 & 1621.9331 & -0.092 & -57 & 367 & 378 \text { AlHLTVPQLTLK } \\ 1622.0251 & 1621.9836 & -0.0415 & -26 & 367 & 378 \text { AlHLTVPQLTLK } \\ 1622.0251 & 1622.0746 & 0.0495 & 31 & 367 & 378 \text { AlHLTVPQLTLK } \\ 1723.9476 & 1723.9762 & 0.0286 & 17 & 379 & 391 \text { ASYDLQDLLAQAK } \\ 1723.9476 & 1723.9945 & 0.0469 & 27 & 379 & 391 \text { ASYDLQDLLAQAK } \\ 1731.9667 & 1731.9246 & -0.0421 & -24 & 347 & 359 \text { VEALIFQHNFLTR } \\ 1761.948 & 1762.0055 & 0.0575 & 33 & 228 & 241 \text { SLDLSTDPNLAAEK } \\ 1878.1 & 1878.0679 & -0.0321 & -17 & 27 & 39 \text { VYIHPFHLLVHSK } \\ 1901.9729 & 1901.9436 & -0.0293 & -15 & 67 & 81 \text { SSPVDEEALWEQLVR } \\ 1909.9365 & 1909.9196 & -0.0169 & -9 & 148 & 164 \text { LQAFLGVPGEGQGCTSR } \\ 2167.3464 & 2167.3809 & 0.0345 & 16 & 193 & 210 \text { LLLSTVVGLFTAPGLHLK } \\ 2213.2039 & 2213.1631 & -0.0408 & -18 & 94 & 110 \text { ASEVGLLLNFMGFHVYK } \\ 2229.1987 & 2229.1565 & -0.0422 & -19 & 94 & 110 \text { ASEVGLLLNFMGFHVYK } \\ & & & & & \\ 2514.4316 & 2514.3342 & -0.0974 & -39 & 325 & 345 \text { VPLSANGYLLLIQPHHTL } \\ 2669.5559 & 2669.6338 & 0.0779 & 29 & 169 & 192 \text { KLR KLSSLQTIQGLLVAPGG }\end{array}$

\begin{tabular}{|c|c|c|c|c|c|c|c|c|}
\hline 52 & 99.815 & (N-term)_iTRAQ[0] & $\begin{array}{l}{[7] F 12 \text { and } F 9 \text { and } F 7} \\
\text { attempt } 2\end{array}$ & 1293/1285 & 0.591 & 0.537 & 0.887 & 1 Mascot \\
\hline 43 & 98.517 & (N-term);iTRAQ[0], & [1] F8 061011 & $154 / 146$ & 0.682 & 0.694 & 0.841 & 1 Mascot \\
\hline 72 & 99.998 & $\begin{array}{l}\text { (N-term) iTRAQI], } \\
\text { Lysine(K) iTRAQ[10] }\end{array}$ & [4] F11 and F3 & 1252/1244 & 2.535 & 2.093 & 1.490 & 1 Mascot \\
\hline 97 & 100 & 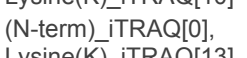 & [4] F11 and F3 & 1174/1166 & 0.968 & 0.778 & 0.947 & 1 Mascot \\
\hline 107 & 100 & 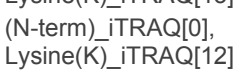 & [5] F5 and F10 & $424 / 416$ & 0.840 & 0.558 & 0.725 & 1 Mascot \\
\hline 61 & 99.976 & $\begin{array}{l}\text { (N-term) ITRAQ[0]] } \\
\text { Lysine(KK) iTAQ[12] }\end{array}$ & [4] F11 and F3 & $344 / 336$ & 1.220 & 1.024 & 1.722 & 1 Mascot \\
\hline 111 & 100 & 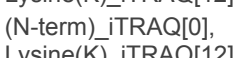 & [3] F $14,15,16$ and $F 6$ & $937 / 929$ & 0.554 & 0.611 & 0.762 & 1 Mascot \\
\hline 98 & 100 & $\begin{array}{l}\text { (N-term) iTRAQI], } \\
\text { Lysine(K) iTRAQ[13] }\end{array}$ & [4] F11 and F3 & 1198/1190 & 0.758 & 0.711 & 0.819 & 1 Mascot \\
\hline 71 & 99.998 & (N-term) TRAQ[0], & [2] F4 and F13 & 276/268 & 0.577 & 0.667 & 0.687 & 1 Mascot \\
\hline 60 & 99.971 & (N-term)_TRAQ[0] & [5] F5 and F10 & $479 / 471$ & 1.725 & 1.608 & 1.243 & 1 Mascot \\
\hline 94 & 100 & $\begin{array}{l}\text { (N-term)(iTRAQ[0], } \\
\text { Lysine(K) iTAQA]4] }\end{array}$ & [4] F11 and F3 & $1058 / 1050$ & 1.260 & 1.073 & 0.994 & 1 Mascot \\
\hline 72 & 99.998 & (N-term) TRAQ[0], & [4] F11 and F3 & $316 / 308$ & 1.129 & 0.852 & 0.943 & 1 Mascot \\
\hline 99 & 100 & (N-term)_iTRAQ[0] & {$[8] \mathrm{F} 2$} & 489/481 & 0.935 & 0.783 & 0.913 & 1 Mascot \\
\hline 79 & 100 & $\begin{array}{l}\text { (N-term) iTRAQ[0], } \\
\text { MMTS (C) C14] }\end{array}$ & {$[8] \mathrm{F} 2$} & $427 / 419$ & 1.359 & 1.156 & 1.126 & 1 Mascot \\
\hline 54 & 99.896 & (N-term) ITRAQ[0], & [2] F4 and F13 & $465 / 457$ & 1.124 & 1.300 & 1.178 & 1 Mascot \\
\hline 127 & 100 & 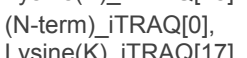 & [5] F5 and F10 & $594 / 586$ & 0.399 & 0.395 & 0.692 & 1 Mascot \\
\hline 52 & 99.822 & 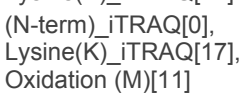 & , ${ }^{[5] \text { F5 and F10 }}$ & $529 / 521$ & 0.765 & 0.722 & 0.892 & 1 Mascot \\
\hline 50 & 99.702 & (N-term)_iTRAQ[0] & [5] F5 and F10 & $491 / 483$ & 0.471 & 0.539 & 0.663 & 1 Mascot \\
\hline 78 & 100 & (N-term & [2] F4 and F13 & $331 / 323$ & 1.167 & 1.186 & 1.097 & 1 Mascot \\
\hline
\end{tabular}

apolipoprotein A-I, apoA-1 [Bos=cattle,
Friesian-Holstein male calves aged 2-4 weeks, Peptide,
247245563 31729.2

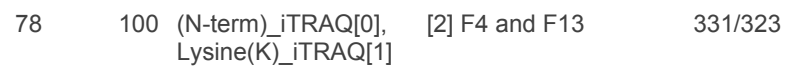

$\begin{array}{llll}1.167 & 1.186 & 1.097 \quad 1 \text { Mascol }\end{array}$

$\begin{array}{lllllllllll}1043 & 0.997 & 0.988 & 1.018 & 0.438 & 0.291 & 0.215 & 27 & 27 & 27 & 100\end{array}$

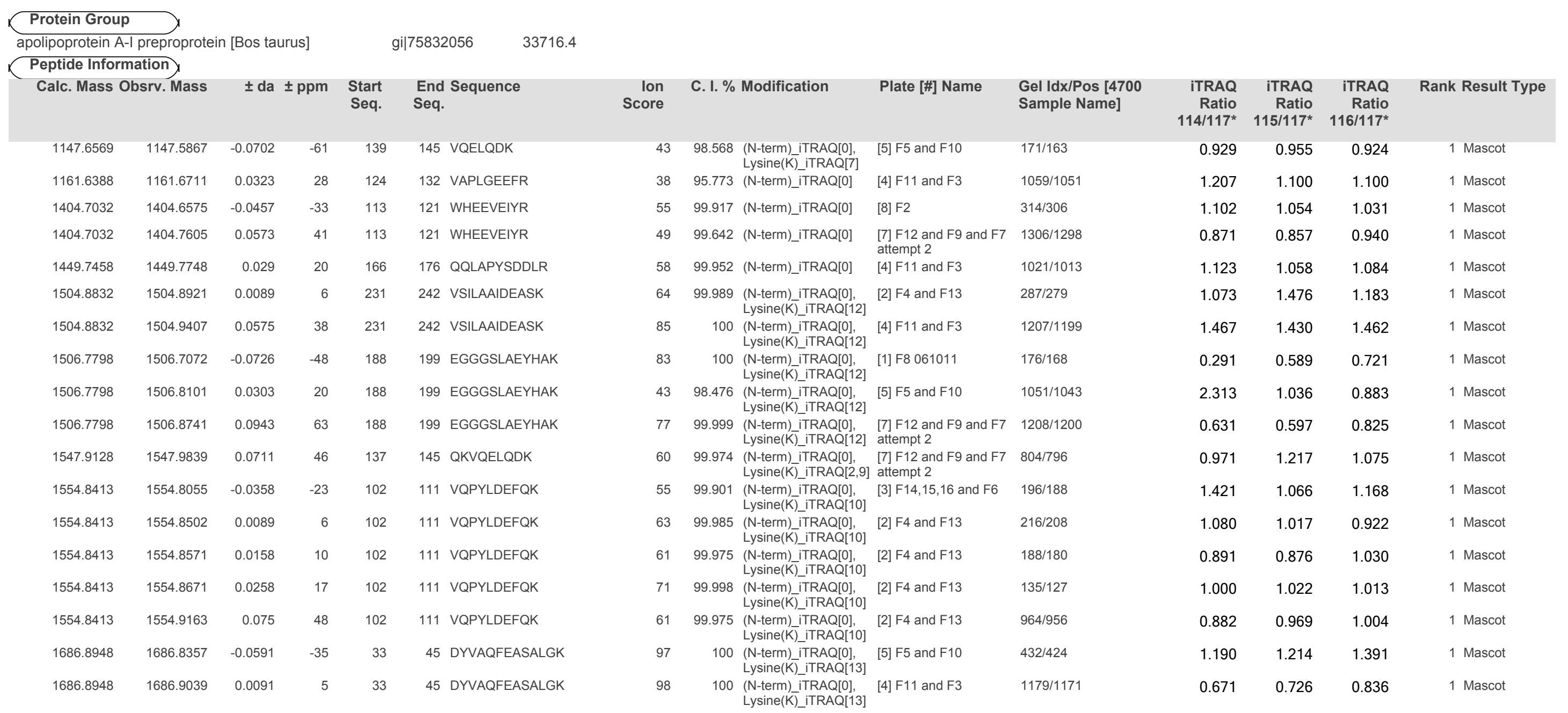


$\begin{array}{llllll}1686.8948 & 1686.9125 & 0.0177 & 10 & 33 & 45 \\ & \text { DVVAQFEASALGK }\end{array}$

$\begin{array}{llllll}1777.0806 & 1777.1949 & 0.1143 & 64 & 231 & 243 \\ \text { VSILAAIDEASKK }\end{array}$

$\begin{array}{llllll}1865.0266 & 1864.9445 & -0.0821 & -44 & 51 & 64 \\ \text { LLDNWDTLASTLSK }\end{array}$

\begin{tabular}{llllll}
1865.0266 & 1865.0361 & 0.0095 & 5 & 51 & 64 \\
\hline
\end{tabular}

$\begin{array}{llllll}1865.0266 & 1865.041 & 0.0144 & 8 & 51 & 64 \\ \text { LLDNWDTLASTLSK }\end{array}$

$\begin{array}{llllll}1915.1276 & 1915.0759 & -0.0517 & -27 & 16 & 28 \\ \text { VKDFATVYVEAIK }\end{array}$

$\begin{array}{llllll}2102.0764 & 2102.1504 & 0.074 & 35 & 29 & 45 \\ \text { DSGRDVVAQFEASALGK }\end{array}$

$\begin{array}{llllll}2221.1387 & 2221.1797 & 0.041 & 18 & 67 & 82 \\ & \text { EQLGPVTQEFWDNLEK }\end{array}$

$\begin{array}{lllllll}2476.3081 & 2476.2375 & -0.0706 & -29 & 65 & 82 \\ \text { VREQLGPVTQEFWDNLE }\end{array}$

$6 \quad$ ceruloplasmin precursor [Ovis aries]
$87 \quad 100$ (N-term) iTRAQ[0], [2] F4 and F13 262/254

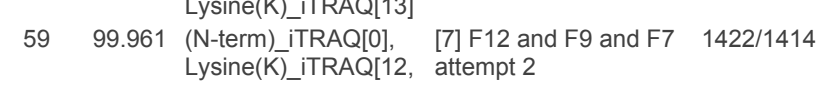

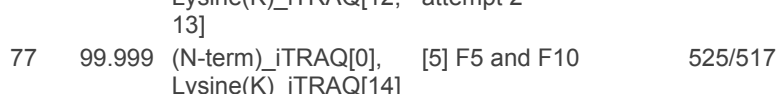

$46 \quad 99.292$ (N-term) iTRAQ[0], [2] F4 and F13 362/354

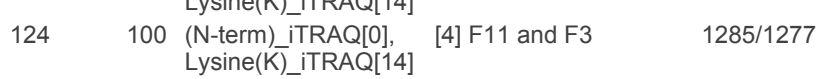

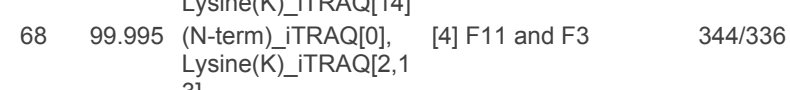

$74 \quad 99.999$ (N-term)_iTRAQ[0], [3] F14,15,16 and F6 931/923

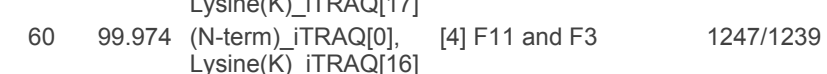

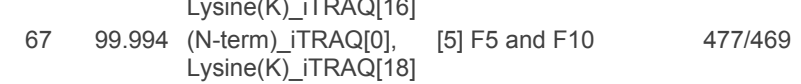

$\begin{array}{llllll}14 & 1038 & 0.850 & 0.840 & 1.154 & 0.342\end{array}$

\begin{tabular}{|c|c|c|c|}
\hline 0.727 & 0.681 & 0.582 & 1 Mascot \\
\hline 1.163 & 1.194 & 1.299 & 1 Masco \\
\hline 1.500 & 1.185 & 1.061 & 1 Mascot \\
\hline 0.995 & 1.113 & 1.094 & 1 Masco \\
\hline 1.116 & 1.116 & 1.051 & 1 Mascot \\
\hline 1.110 & 0.629 & 1.317 & 1 Masco \\
\hline 1.056 & 1.331 & 0.977 & 1 Mascot \\
\hline 0.628 & 0.698 & 0.928 & 1 Masco \\
\hline 1.186 & 1.309 & 1.085 & 1 Mascc \\
\hline & & & \\
\hline
\end{tabular}

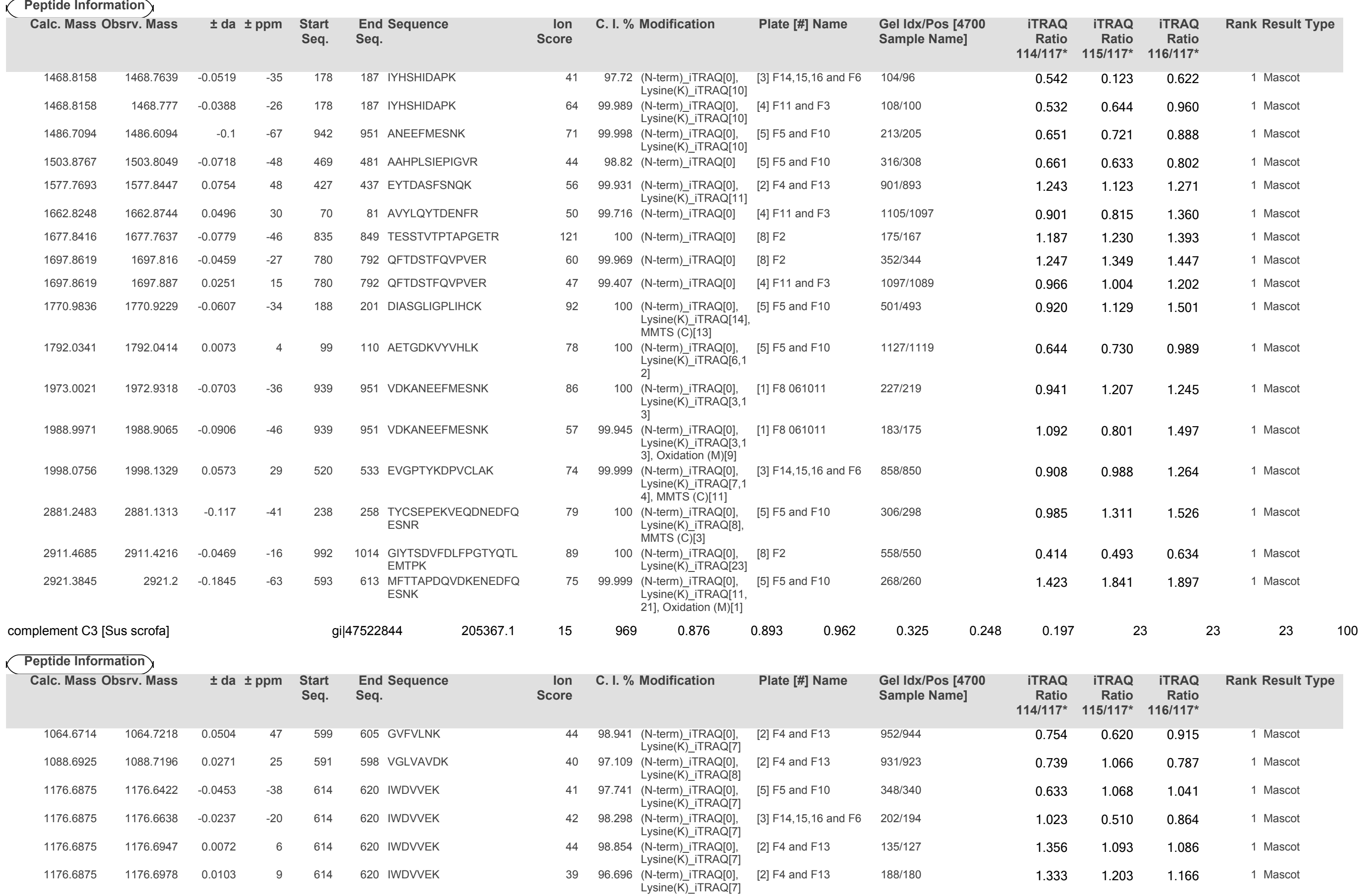




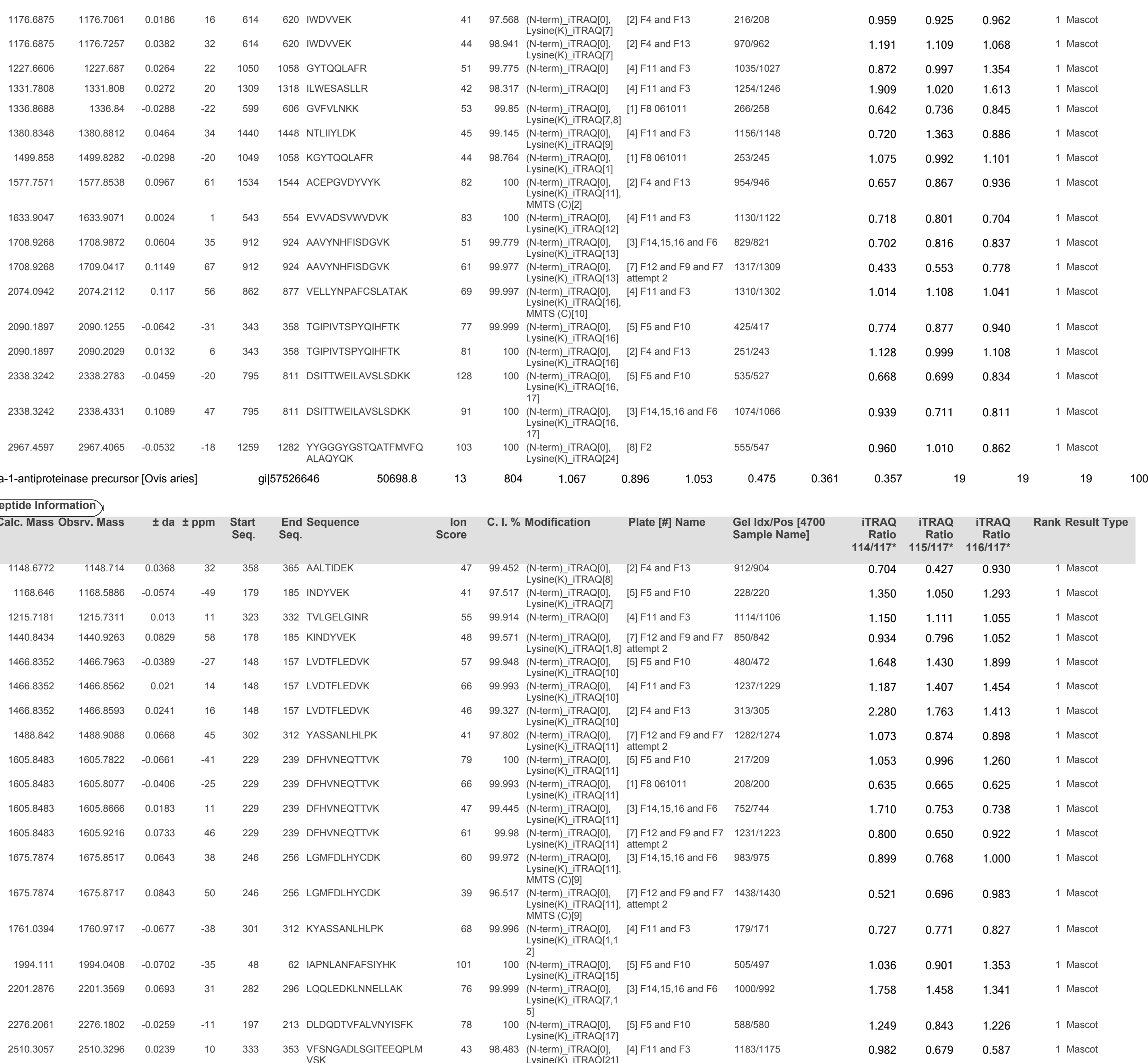




\begin{tabular}{|c|c|c|c|c|c|c|c|c|c|c|c|c|c|}
\hline Calc. Mass C & srv. Mass & \pm da & ppm & $\begin{array}{l}\text { Start } \\
\text { Seq. }\end{array}$ & $\begin{array}{l}\text { End Sequence } \\
\text { Seq. }\end{array}$ & $\begin{array}{l}\text { lon } \\
\text { Score }\end{array}$ & C. I. \% Modification & Plate [\#] Name & $\begin{array}{l}\text { Gel Idx/Pos [4700 } \\
\text { Sample Name] }\end{array}$ & $\begin{array}{r}\text { iTRAQ } \\
\text { Ratio } \\
114 / 117^{*}\end{array}$ & $\begin{array}{r}\text { iTRAQ } \\
\text { Ratio } \\
115 / 17^{*}\end{array}$ & $\begin{array}{r}\text { iTRAQ } \\
\text { Ratio } \\
116 / 117^{*}\end{array}$ & Rank Result Type \\
\hline 1177.6449 & 1177.6891 & 0.0442 & 38 & 269 & 277 LQAEAFQAR & 53 & 99.845 (N-term)_iTRAQ[0] & [4] F11 and F3 & 1006/998 & 1.316 & 1.075 & 1.288 & 1 Mascot \\
\hline 1179.58 & 1179.6038 & 0.0238 & 20 & 121 & 129 LGSDMEDLR & 57 & 99.946 (N-term)_iTRAQ[0] & [4] F11 and F3 & 1037/1029 & 1.100 & 1.022 & 0.981 & 1 Mascot \\
\hline 1218.7092 & 1218.6534 & -0.0558 & -46 & 292 & 299 QWAGLVEK & 60 & 99.973 (N-term) iTRAQ[0], & [5] F5 and F10 & $310 / 302$ & 0.678 & 1.182 & 1.086 & 1 Mascot \\
\hline 1218.7092 & 1218.7697 & 0.0605 & 50 & 292 & 299 QWAGLVEK & 58 & 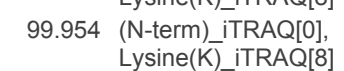 & [2] F4 and F13 & $950 / 942$ & 1.438 & 1.271 & 1.286 & 1 Mascot \\
\hline 1565.8043 & 1565.7266 & -0.0777 & -50 & 176 & 189 LAVYQAGASEGAER & 59 & 99.963 (N-term)_TRAQ[0] & [5] F5 and F10 & $248 / 240$ & 1.470 & 0.659 & 1.089 & 1 Mascot \\
\hline 1565.8043 & 1565.7374 & -0.0669 & -43 & 176 & 189 LAVYQAGASEGAER & 78 & 100 (N-term)_TRAQ[0] & [8] F2 & $256 / 248$ & 1.461 & 1.193 & 1.137 & 1 Mascot \\
\hline 1565.8043 & 1565.8868 & 0.0825 & 53 & 176 & 189 LAVYQAGASEGAER & 86 & 100 (N-term)_iTRAQ[0] & [4] F11 and F3 & 999/991 & 1.219 & 1.103 & 1.203 & 1 Mascot \\
\hline 1643.8262 & 1643.8535 & 0.0273 & 17 & 37 & 49 GQDSQPWEQVLGR & 51 & 99.775 (N-term)_iTRAQ[0] & [4] F11 and F3 & $1114 / 1106$ & 0.735 & 0.787 & 0.953 & 1 Mascot \\
\hline 1680.8176 & 1680.7826 & -0.035 & -21 & 280 & 291 SWFEPLVEDMQR & 71 & 99.998 (N-term)_iTRAQ[0] & [8] F2 & $498 / 490$ & 0.898 & 0.773 & 0.963 & 1 Mascot \\
\hline 1713.9619 & 1713.9227 & -0.0392 & -23 & 209 & 223 AATLSTQVGQPLLDR & 75 & 99.999 (N-term)_iTRAQ[0] & [8] F2 & $339 / 331$ & 1.030 & 0.894 & 1.055 & 1 Mascot \\
\hline 1821.9137 & 1821.8862 & -0.0275 & -15 & 137 & 151 SEVQAMLGQSTEELR & 98 & 100 (N-term)_iTRAQ[0] & [8] F2 & $387 / 379$ & 1.295 & 1.090 & 1.204 & 1 Mascot \\
\hline 1837.9086 & 1837.8442 & -0.0644 & -35 & 137 & 151 SEVQAMLGQSTEELR & 53 & 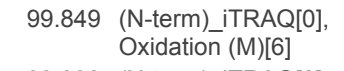 & {$[8] \mathrm{F} 2$} & $290 / 282$ & 1.014 & 0.922 & 0.964 & 1 Mascot \\
\hline 2045.0093 & 2044.9668 & -0.0425 & -21 & 93 & 109 EELEGQLAPMAQETQAR & 73 & 99.999 (N-term)_iTRAQ[0] & [8] F2 & $323 / 315$ & 0.660 & 0.829 & 1.331 & 1 Mascot \\
\hline 2435.2109 & 2435.2227 & 0.0118 & 5 & 90 & 109 AYREELEGQLAPMAQET & 63 & 99.987 (N-term)_iTRAQ[0] & [2] F4 and F13 & 188/180 & 1.015 & 0.975 & 0.886 & 1 Mascot \\
\hline 2435.2109 & 2435.2546 & 0.0437 & 18 & 90 & 109 AYREELEGQLAPMAQET & 47 & 99.406 (N-term)_iTRAQ[0] & [2] F4 and F13 & $135 / 127$ & 0.973 & 0.896 & 0.764 & 1 Mascot \\
\hline 2451.2058 & 2451.2578 & 0.052 & 21 & 90 & $\begin{array}{l}109 \text { AYREELEGQLAPMAQET } \\
\text { QAR }\end{array}$ & 49 & $\begin{array}{l}99.612 \text { (N-term)_iTRAQ[0], } \\
\text { Oxidation (M)[13] }\end{array}$ & [2] F4 and F13 & $135 / 127$ & 1.703 & 0.830 & 1.001 & 1 Mascot \\
\hline
\end{tabular}

10 complement component C3 [Ovis aries]

$$
\text { gi|12649541 } 43395
$$

$\begin{array}{lllll}10 & 576 & 0.956 & 0.911 & 1.015\end{array}$

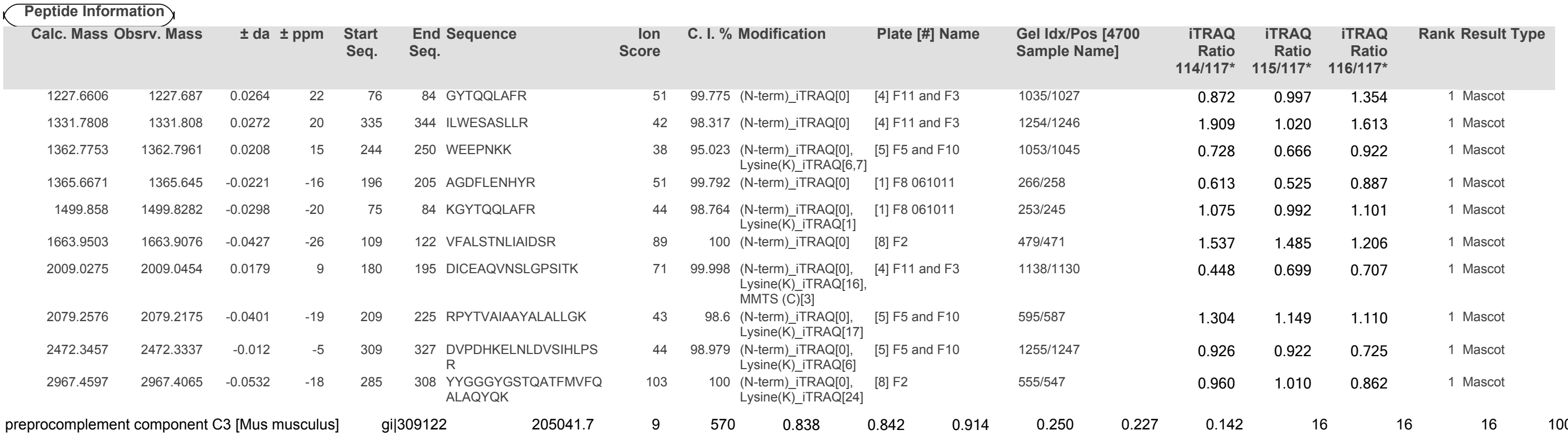

11 preprocomplement component C3 [Mus musculus] gi|309122 205041.7 RecName: Full=Complement C3; Contains: RecName: gi|116597 RecName: Full=Complement C3; Contains: RecName:
Full=Complement C3 beta chain; Contains: RecName: $\begin{array}{lll}\mathrm{F} & & \\ \text { complement C3 [Mus musculus] } & \text { gil126518317 } & 204899.5 \\ \text { complement component C3 prepropeptide, last [Mus } & \text { gil387114 } & 205042.7\end{array}$ musculus]
Peptide Information

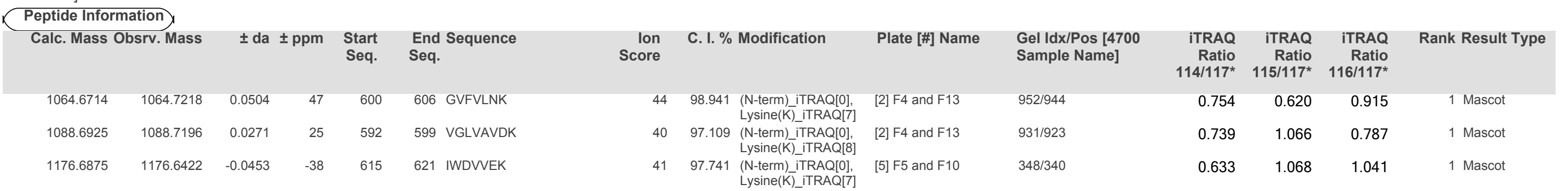




$\begin{array}{rrrrrl}1176.6875 & 1176.6638 & -0.0237 & -20 & 615 & 621 \text { IWDVVEK } \\ 1176.6875 & 1176.6947 & 0.0072 & 6 & 615 & 621 \text { IWDVVEK } \\ 1176.6875 & 1176.6978 & 0.0103 & 9 & 615 & 621 \text { IWDVVEK } \\ 1176.6875 & 1176.7061 & 0.0186 & 16 & 615 & 621 \text { IWDVVEK } \\ 1176.6875 & 1176.7257 & 0.0382 & 32 & 615 & 621 \text { IWDVVEK } \\ 1336.8688 & 1336.84 & -0.0288 & -22 & 600 & 607 \text { GVFVLNKK } \\ 1577.7571 & 1577.8538 & 0.0967 & 61 & 1536 & 1546 \text { ACEPGVDYVYK } \\ 1633.9047 & 1633.9071 & 0.0024 & 1 & 545 & 556 \text { EVVADSVWVDVK } \\ 1660.0295 & 1660.0381 & 0.0086 & 5 & 892 & 904 \text { SSVAVPYVIVPLK } \\ 1999.0331 & 1999.007 & -0.0261 & -13 & 1492 & 1504 \text { FYHPEKDDGMLSK } \\ 2015.028 & 2015.0646 & 0.0366 & 18 & 1492 & 1504 \text { FYHPEKDDGMLSK } \\ 2338.3242 & 2338.2783 & -0.0459 & -20 & 797 & 813 \text { DSITTWELLAVSLSDKK } \\ 2338.3242 & 2338.4331 & 0.1089 & 47 & 797 & 813 \text { DSITTWEILAVSLSDKK }\end{array}$

\begin{tabular}{|c|c|c|c|c|c|c|c|c|}
\hline 42 & 98.298 & (N-term) ITRAQ[0], & {$[3] \mathrm{F} 14,15,16$ and $F 6$} & 202/194 & 1.023 & 0.510 & 0.864 & 1 Mascot \\
\hline 44 & 98.854 & 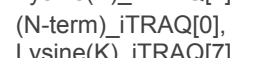 & [2] F4 and F13 & 135/127 & 1.356 & 1.093 & 1.086 & 1 Mascot \\
\hline 39 & 96.696 & 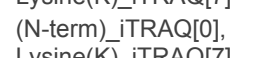 & [2] F4 and F13 & 188/180 & 1.333 & 1.203 & 1.166 & 1 Mascot \\
\hline 41 & 97.568 & $\begin{array}{l}\text { (N-term) iTRAQ[O], } \\
\text { Lysine(K) iTRAQT7] }\end{array}$ & [2] F4 and F13 & $216 / 208$ & 0.959 & 0.925 & 0.962 & 1 Mascot \\
\hline 44 & 98.941 & $\begin{array}{l}\text { (N-term) TRAQ[0] } \\
\text { (T) }\end{array}$ & [2] F4 and F13 & 970/962 & 1.191 & 1.109 & 1.068 & 1 Mascot \\
\hline 53 & 99.85 & $\begin{array}{l}\text { (N-term) iTRAQ[0], } \\
\text { Lysine(K) iTRAQ7,8] }\end{array}$ & [1] F8 061011 & $266 / 258$ & 0.642 & 0.736 & 0.845 & 1 Mascot \\
\hline 82 & 100 & $\begin{array}{l}\text { (N-term) TTRAQ[0], } \\
\text { Lysine(K),iTRAQ[11, } \\
\text { MMTS(C)I2] }\end{array}$ & [2] F4 and F13 & $954 / 946$ & 0.657 & 0.867 & 0.936 & 1 Mascot \\
\hline 83 & 100 & $\begin{array}{l}\text { (N-term) iTRAQ[0], } \\
\text { Lysine(K) iTRAO[12] }\end{array}$ & [4] F11 and F3 & 1130/1122 & 0.718 & 0.801 & 0.704 & 1 Mascot \\
\hline 52 & 99.813 & $\begin{array}{l}\text { (N-term) iTRAQ[0], } \\
\text { ivsino(K) }\end{array}$ & [4] F11 and F3 & $1187 / 1179$ & 0.971 & 1.016 & 0.720 & 1 Mascot \\
\hline 44 & 98.979 & $\begin{array}{l}\text { (N-term) iTRAQ[0] } \\
\text { Lysine(K)_iTRAQ[6, } \\
3]\end{array}$ & [5] F5 and F10 & $1150 / 1142$ & 0.666 & 0.713 & 1.031 & 1 Mascot \\
\hline 44 & 98.822 & 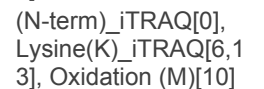 & [5] F5 and F10 & 1110/1102 & 0.651 & 0.714 & 0.998 & 1 Mascot \\
\hline 128 & 100 & $\begin{array}{l}\text { (N-term)iTRAQ[0], } \\
\text { Lysine(K)_iTRAQ[16, } \\
17]\end{array}$ & [5] F5 and F10 & $535 / 527$ & 0.668 & 0.699 & 0.834 & 1 Mascot \\
\hline 91 & 100 & $\begin{array}{l}\text { (N-term)iTRAQ[0], } \\
\text { Lysine(K)_iTRAQ[16, }\end{array}$ & {$[3] \mathrm{F} 14,15,16$ and $\mathrm{F} 6$} & 1074/1066 & 0.939 & 0.711 & 0.811 & 1 Mascot \\
\hline
\end{tabular}

12 transferrin [Sus scrofa] $\quad$ gi|833800 $\quad 86770.7$

$549-0.080-0777-0.855-0305$

0.076

Protein Group
RecName: Full=Serotransferrin; Short=Transferrin; $\quad$ gil136192 86931.9
AltName: Full=Beta-1 metal-binding globulin; AltN
Peptide Information
AltName: Full=Beta-1 me
Peptide Information
Calc. Mass Obsrv. Mass

\begin{tabular}{|c|c|c|c|c|c|}
\hline \multicolumn{2}{|c|}{ Calc. Mass Obsrv. Mass } & \multicolumn{2}{|c|}{ \pm da $\pm \mathrm{ppm}$} & $\begin{array}{l}\text { Start } \\
\text { Seq. }\end{array}$ & \multirow[t]{2}{*}{$\begin{array}{l}\text { End Sequence } \\
\text { Seq. }\end{array}$} \\
\hline 1023.6084 & 1023.5566 & -0.0518 & -51 & 537 & \\
\hline 1207.766 & 1207.7739 & 0.0079 & 7 & 430 & 438 GYLAVAVVK \\
\hline 1241.6075 & 1241.6476 & 0.0401 & 32 & 523 & 531 YYGYTGAFR \\
\hline 1639.8778 & 1639.9443 & 0.0665 & 41 & 406 & 417 CGLVPVLAENYK \\
\hline 1893.0116 & 1892.9187 & -0.0929 & -49 & 88 & 101 DNPQTHYYAVAVVK \\
\hline 1893.0116 & 1892.9353 & -0.0763 & -40 & 88 & 101 DNPQTHYYAVAVVK \\
\hline 1893.0116 & 1892.9382 & -0.0734 & -39 & 88 & 101 DNPQTHYYAVAVVK \\
\hline 1893.0116 & 1892.9613 & -0.0503 & -27 & 88 & 101 DNPQTHYYAVAVVK \\
\hline 1893.0116 & 1892.9622 & -0.0494 & -26 & 88 & 101 DNPQTHYYAVAVVK \\
\hline 1893.0116 & 1893.058 & 0.0464 & 25 & 88 & 101 DNPQTHYYAVAVVK \\
\hline 1893.0116 & 1893.074 & 0.0624 & 33 & 88 & 101 DNPQTHYYAVAVVK \\
\hline 2119.0627 & 2118.9917 & -0.071 & -34 & 388 & 405 GEADAMSLDGGYYYIAGK \\
\hline 2119.0627 & 2119.0623 & -0.0004 & 0 & 388 & 405 GEADAMSLDGGYIYIAGK \\
\hline 2603.4126 & 2603.3179 & -0.0947 & -36 & 385 & $\begin{array}{l}405 \text { IVKGEADAMSLDGGYIYIA } \\
\text { GK }\end{array}$ \\
\hline 2603.4126 & 2603.4468 & 0.0342 & 13 & 385 & $\begin{array}{l}405 \text { IVKGEADAMSLDGGYYYIA } \\
\text { GK }\end{array}$ \\
\hline 2619.4075 & 2619.3086 & -0.0989 & -38 & 385 & $\begin{array}{l}405 \text { IVKGEADAMSLDGGYIYIA } \\
\text { GK }\end{array}$ \\
\hline
\end{tabular}

C. $1 . \%$ Modication

iTRAQ ITRAQ iTRAQ Rank Result Type

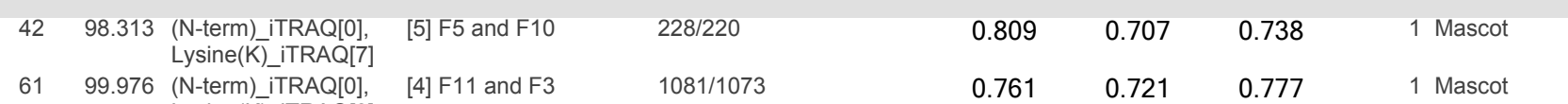

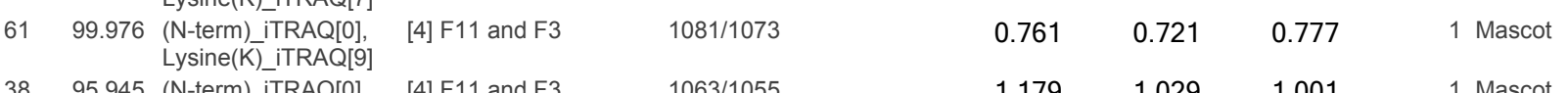

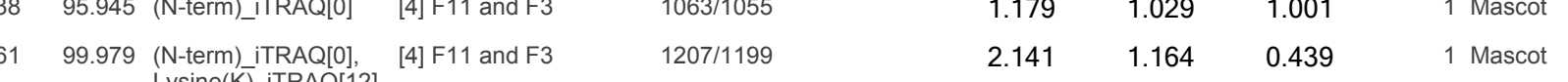

Lysine(K) $(K)$ iTRAQ[12],
MMTS (C)[1]

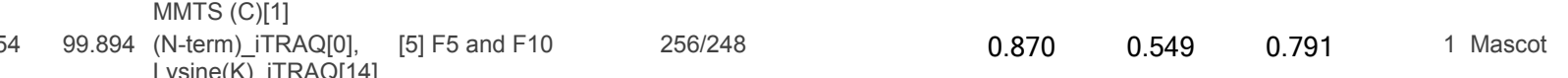

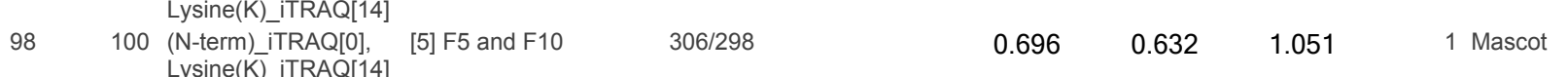

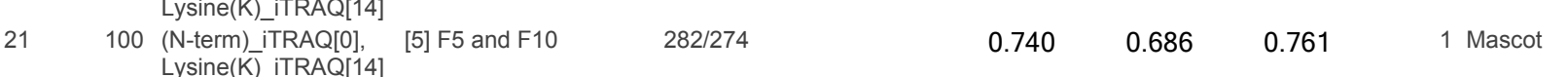

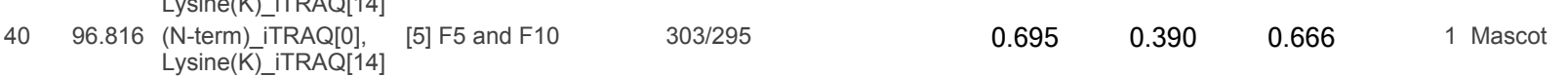

91100 (N-term) iTRAQ[0], [1] F8 061011 $2699 / 261 \quad 1.034 \quad 1.017 \quad 0.774 \quad 1$ Mascot

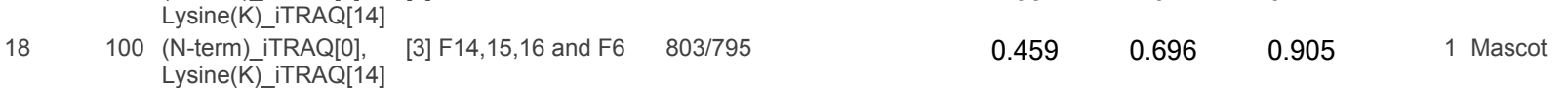

100 (N-term) iTRAQ[0], [5] F5 and F10 $1153 / 1145 \quad 0.746 \quad 0.829 \quad 1.022 \quad 1$ Mascot

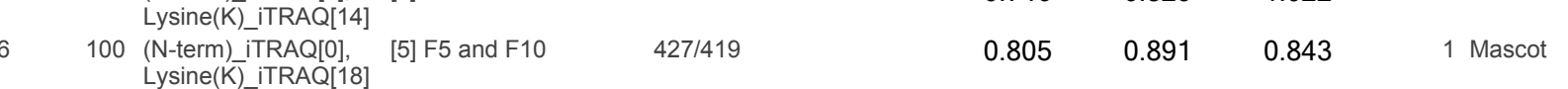

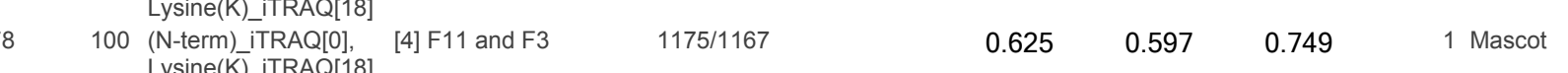

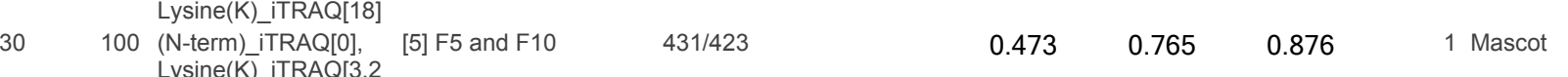

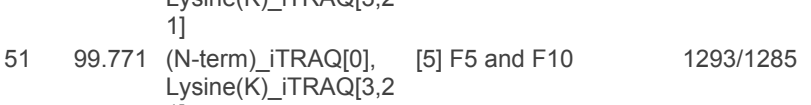

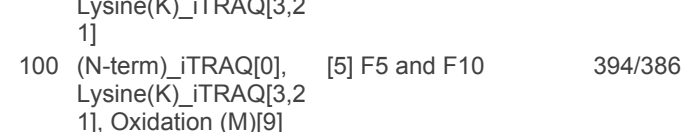

$\begin{array}{llll}0.609 & 0.977 \quad 1.343 \quad 1 \text { Mascot }\end{array}$

$\begin{array}{llll}1.330 & 1.333 & 1.442 \quad 1 \text { Mascot }\end{array}$

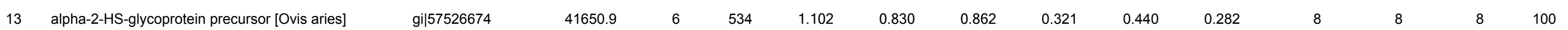
Peptide Information

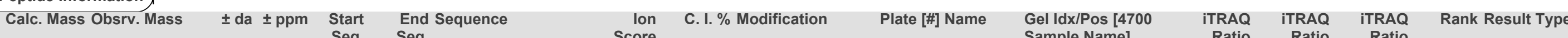

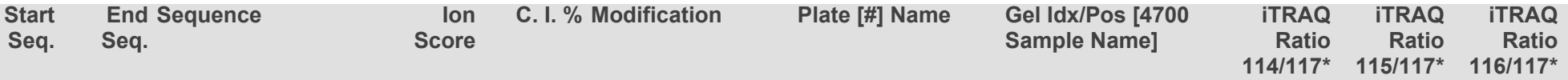




\begin{abstract}
$\begin{array}{llllll}1442.8213 & 1442.788 & -0.0333 & -23 & 58 & 67 \\ \text { HTLNQIDSVK }\end{array}$
$\begin{array}{lllllll}1557.8523 & 15578696 & 0.0173 & 11 & 121 & 131 & \text { ODGOFSVFTK }\end{array}$

$\begin{array}{lllllll}1557.8523 & 1557.9008 & 0.0485 & 31 & 121 & 131 & \text { QDGQFSVLFTK }\end{array}$

$\begin{array}{llllll}2256.0979 & 2256.0513 & -0.0466 & -21 & 104 & 120 \\ \text { QQTEHAVEGDCDIHVLK }\end{array}$

$\begin{array}{lllllll}2392.2141 & 2392.1865 & -0.0276 & -12 & 318 & 338 \text { HTFSGVASVESASGEAF } \\ \text { HVGK }\end{array}$

$\begin{array}{lllllll}2683.2458 & 2683.1899 & -0.0559 & -21 & 29 & \begin{array}{c}\text { HVGK } \\ \text { EPACDDDTEQAALAAV } \\ \text { DYINK }\end{array}\end{array}$

$\begin{array}{llllll}2683.2458 & 2683.2727 & 0.0269 & 10 & 29 & 50 \begin{array}{l}\text { EPACDDPDTEQAALAAV } \\ \text { DYINK }\end{array}\end{array}$

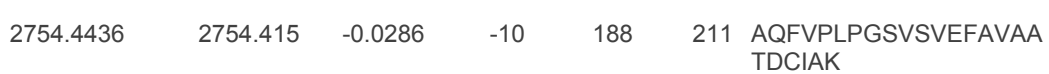

$76 \quad 99.999$ (N-term)itRAQ[0], [1] F8 $061011 \quad 213 / 205$

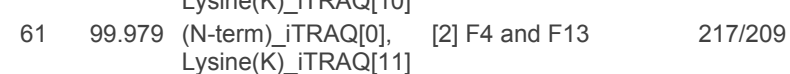

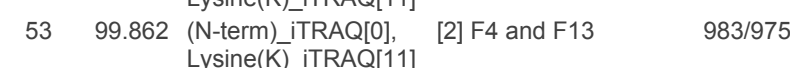

$7799.999 \begin{aligned} & \text { N-term) iTRAQ[1], } \\ & \text { Lysine(K) TRRAQ[1]] }\end{aligned}$ [1] F8 061011 Lysine(K) RARAQ[1],
MMTS (C) [11]

$\left.60 \quad 99.973 \begin{array}{l}\text { MN-term) }(\text { iTRAQ[0], } \\ \text { Lysine(K) iRRAQ[21] }\end{array}\right]$ [1] F8 061011

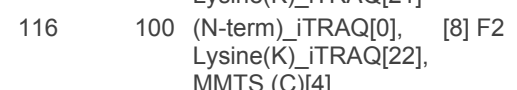

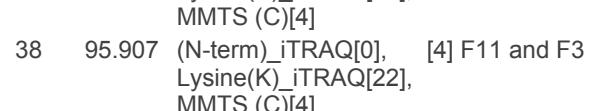

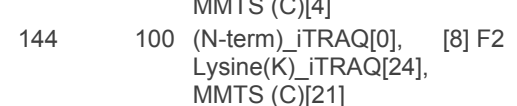

$587 / 579$

$\begin{array}{llll}1.130 & 0.970 & 1.063 & 1 \text { Mascot } \\ 1.646 & 1.445 & 1.267 & 1 \text { Mascot } \\ 1.578 & 1.205 & 0.838 & 1 \text { Mascot } \\ 1.075 & 0.893 & 1.152 & 1 \text { Mascot } \\ 0.971 & 0.891 & 0.964 & 1 \text { Mascot } \\ 0.881 & 0.317 & 0.732 & 1 \text { Mascot } \\ 1.102 & 0.772 & 0.577 & 1 \text { Mascot } \\ 0.734 & 0.686 & 0.576 & 1 \text { Mascot }\end{array}$

0.734

14 immunoglobulin lambda light chain constant region $\quad$ gi|52366986 $\quad 13044.6$

\section{Peptide Information}

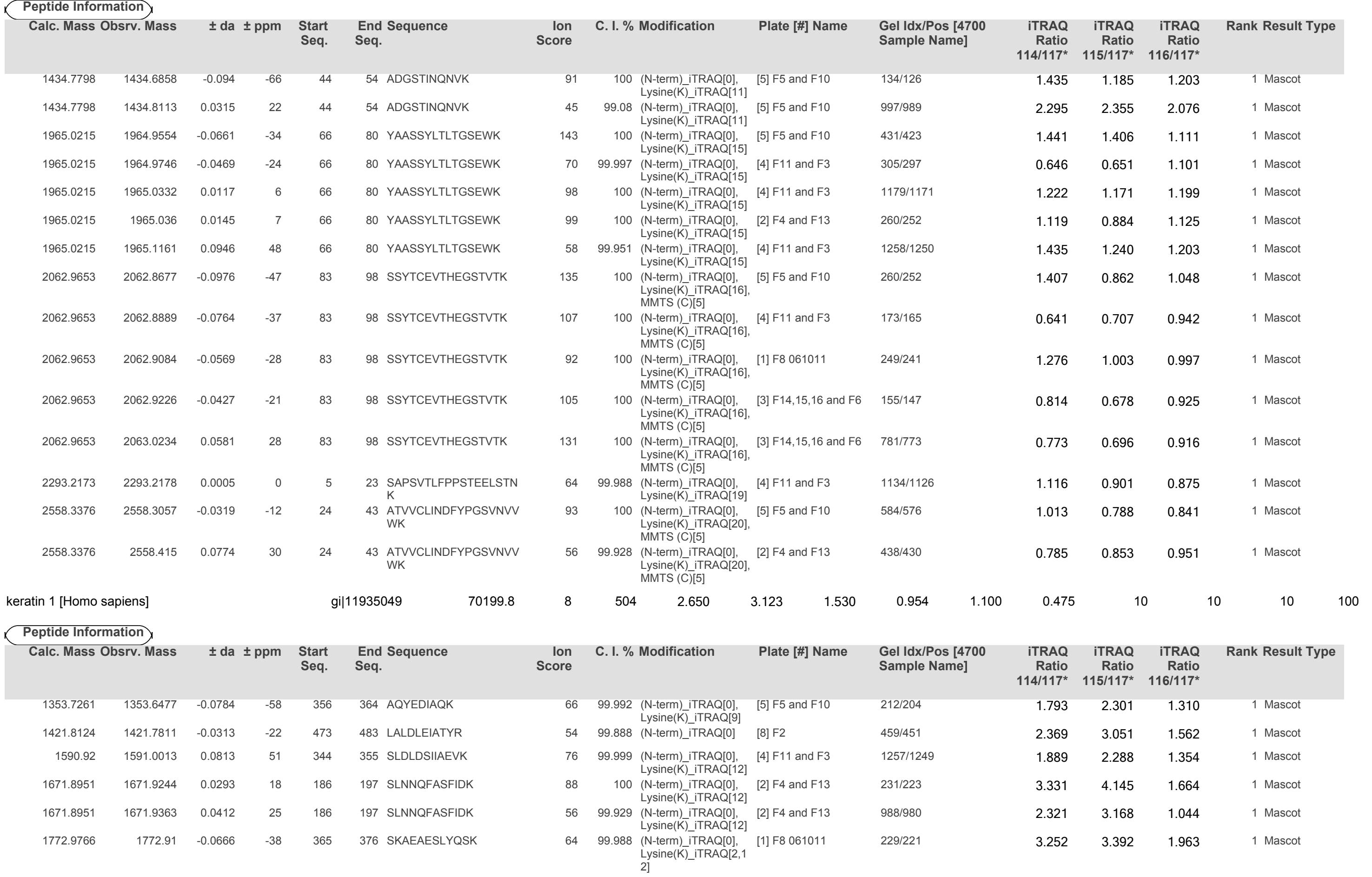


$\begin{array}{llllll}1772.9766 & 1772.9552 & -0.0214 & -12 & 365 & 376 \\ & \text { SKAEAESLYQSK }\end{array}$

$\begin{array}{llllll}1826.0396 & 1826.1379 & 0.0983 & 54 & 278 & 289 \\ \text { TNAENEFVTIKK }\end{array}$

$\begin{array}{llllll}1860.9536 & 1860.9867 & 0.0331 & 18 & 418 & 432 \\ \text { QISNLQQSISDAEQR }\end{array}$

$2138.0791 \quad 2138.0222 \quad-0.0569 \quad-27 \quad 224 \quad 239$ THNLEPYFESFINNLR

16 alpha-1-antiproteinase precursor [Bos taurus] gi|27806941 51106.

Peptide Information
Calc. Mass Obsrv. Mass \pm da \pm ppm Start End Sequence

$\begin{array}{llllll}1148.6772 & 1148.714 & 0.0368 & 32 & 358 & 365 \\ \text { AALTIDEK }\end{array}$

$\begin{array}{rrrrrl}1168.646 & 1168.5886 & -0.0574 & -49 & 179 & 185 \text { INDYVEK } \\ 14408434 & 1440.9263 & 0.0829 & 58 & 178 & 185 \text { KINDYVEK }\end{array}$

$\begin{array}{lllllll}1440.8434 & 1440.9263 & 0.0829 & 58 & 178 & 185 & \text { KINDYVEK }\end{array}$

$\begin{array}{rrrrrr}1466.8352 & 1466.7963 & -0.0389 & -27 & 148 & 157 \text { LVDTFLEDVK } \\ 1466.8352 & 1466.8562 & 0.021 & 14 & 148 & 157 \text { LVDTFLEDVK }\end{array}$

$\begin{array}{llllll}1466.8352 & 1466.8593 & 0.0241 & 16 & 148 & 157 \\ \text { LVDTFLEDVK }\end{array}$

$\begin{array}{llllll}1488.842 & 1488.9088 & 0.0668 & 45 & 302 & 312 \\ \text { YASSANLHLPK }\end{array}$

\begin{tabular}{llllll}
1600.8323 & 1606.866 & 0.0337 & 21 & 229 & 239 \\
\hline
\end{tabular}

$\begin{array}{lllllll}1606.8323 & 1606.9128 & 0.0805 & 50 & 229 & 239 & \text { DFHVDEQTTVK }\end{array}$

$\begin{array}{lllllll}1675.7874 & 1675.8517 & 0.0643 & 38 & 246 & 256 & \text { LGMFDLHYCDK }\end{array}$

$\begin{array}{lllllll}1675.7874 & 1675.8717 & 0.0843 & 50 & 246 & 256 & \text { LGMFDLHYCDK }\end{array}$

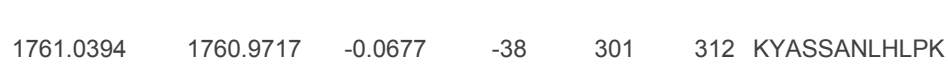

$\begin{array}{llllll}2201.2876 & 2201.3569 & 0.0693 & 31 & 282 & 296 \\ & \text { LQQLEDKLNNELLAK }\end{array}$

$\begin{array}{rrrr}\begin{array}{r}\text { lon } \\ \text { Score }\end{array} & \text { C. I. \% Modification } & \text { Plate [\#] Name } & \begin{array}{l}\text { Gel Idx/Pos } \\ \text { Sample Nam }\end{array} \\ 47 & 99.452 \text { (N-term) iTRAQ[0]. } & {[2] \text { F4 and F13 }} & 912 / 904\end{array}$

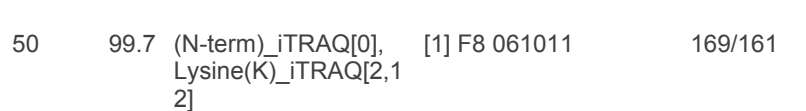

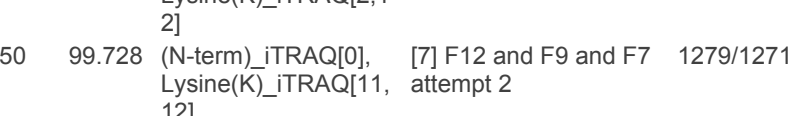

5599.909 (N-term)_iTRAQ[0] [4] F11 and F3 1071/1063

5299.838 (N-term)_iTRAQ[0] $[5]$ F5 and F10 $546 / 538$

$\begin{array}{cccc}1.755 & 1.828 & 1.161 & 1 \text { Mascot } \\ 3.007 & 3.023 & 1.254 & 1 \text { Mascot } \\ & & & 1 \text { Mascot } \\ 3.568 & 4.925 & 2.679 & 1 \text { Mascot } \\ 4.493 & 4.529 & 1.904 & 13 \\ 0.354 & 13 & 13 & 13\end{array}$

100

iTRAQ ITRAQ ITRAQ Rank Result Type

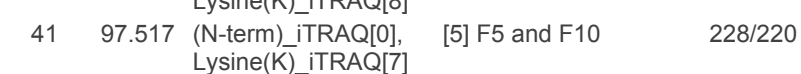

$48 \quad 99.571$ (N-term) - TTRAQ[0], $[7]$ F12 and F9 and F7 $850 / 842$

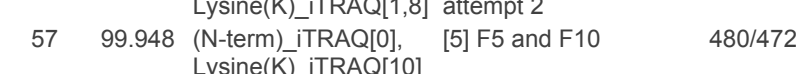

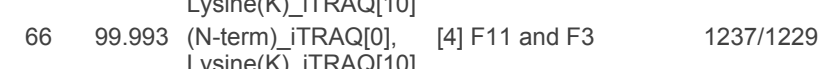

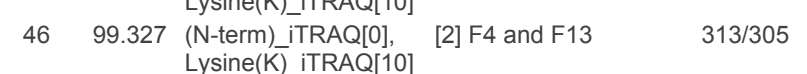

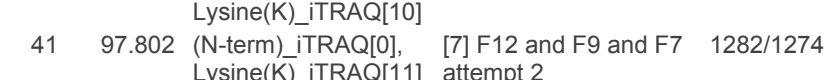

$5599.9 \begin{aligned} & \text { (N-term) iTRAQ[0], } \\ & \text { Lysine(K)] }\end{aligned}$

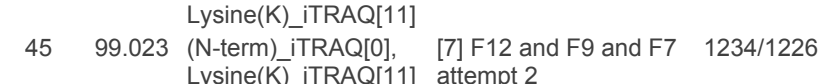

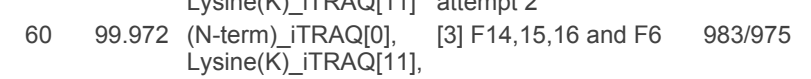

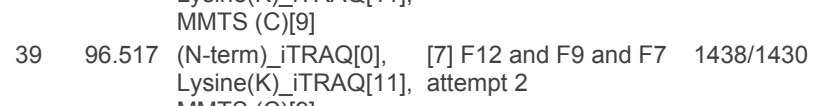

$68 \quad 99.996 \begin{gathered}\text { MMTS (C)[9] } \\ \text { (N-term) iTRAQ[0], } \\ \text { Lysine(K) iTRAQ[1,1 }\end{gathered}$ [4] F11 and F3 179/171

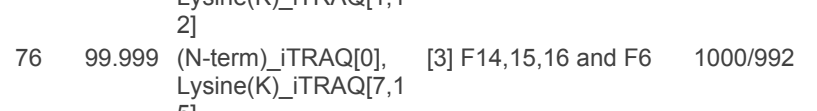

$\begin{array}{lll}0.704 & 0.427 & 0.930\end{array}$

$\begin{array}{lll}1.350 & 1.050-1.293 & 1 \text { Mascot }\end{array}$

$\begin{array}{llll}0.934 & 0.796 & 1.052 & 1 \text { Mascot }\end{array}$

$\begin{array}{llll}1.648 & 1.430 & 1.899 & 1 \text { Mascot }\end{array}$

$\begin{array}{llll}1.187 & 1.407 & 1.454 & 1 \text { Mascot }\end{array}$

$\begin{array}{llll}2.280 & 1.763 & 1.413 & 1 \text { Mascot }\end{array}$

$\begin{array}{llll}1.073 & 0.874 & 0.898 & 1 \text { Mascot }\end{array}$

$\begin{array}{llll}0.819 & 0.623 & 0.745 & 1 \text { Mascot }\end{array}$

$\begin{array}{llll}0.708 & 0.616 & 0.675 & 1 \text { Mascot }\end{array}$

$\begin{array}{llll}0.899 & 0.768 & 1.000 \quad 1 \text { Mascot }\end{array}$

$\begin{array}{llll}0.521 & 0.696 & 0.983 & 1 \text { Mascot }\end{array}$

$\begin{array}{lll}0.727 & 0.771 & 0.827\end{array} \quad 1$ Mascot

$\begin{array}{llll}1.758 & 1.458 & 1.341 & 1 \text { Mascot }\end{array}$

17 Ig lambda chain C region - sheep (fragment) gil109030 13171.8

Peptide Information

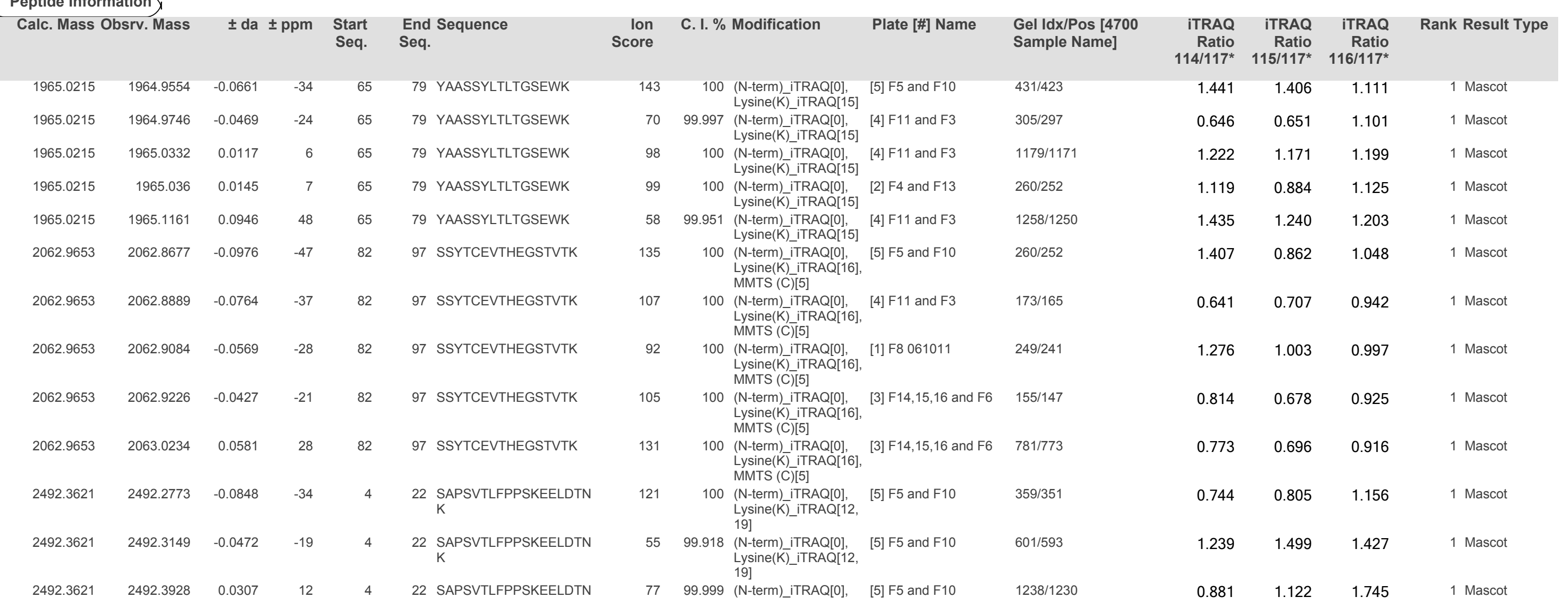




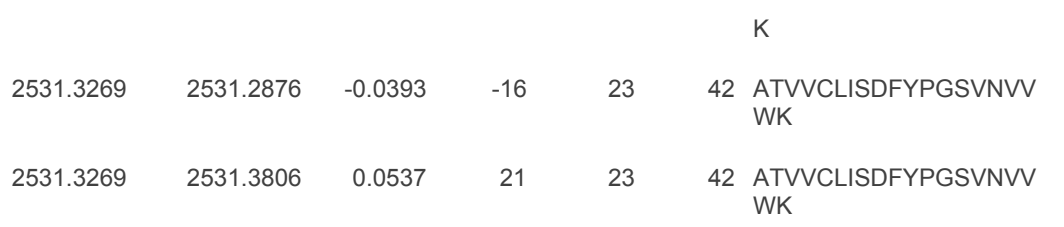

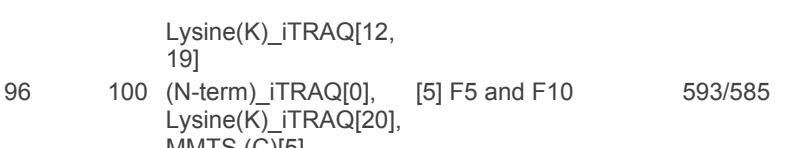

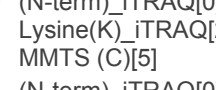

$46 \quad 99.311$ ( $\left.\begin{array}{l}\text { (N-term) iTRAQ[0], } \\ \text { Lysine(K) }(\text { iTRAQ[20], }\end{array}\right]$ F4 and F13 446/438 $446 / 438$ $\begin{array}{llll}0.362 & 0.502 & 1.034 & 1 \text { Mascot }\end{array}$ Lysine(K) $($ )iTRA
MMTS (C) [5]

18 complement C3 preproprotein [Cavia porcellus] $\quad$ gil290543340 204039.3

$\begin{array}{lll}483 & 0.868 & 0.896\end{array}$

$\begin{array}{lll}0.915 & 0.259 & 0.280\end{array}$

$\begin{array}{llll}0.646 & 0.842 & 1.023 & 1 \text { Mascot }\end{array}$

Peptide Information

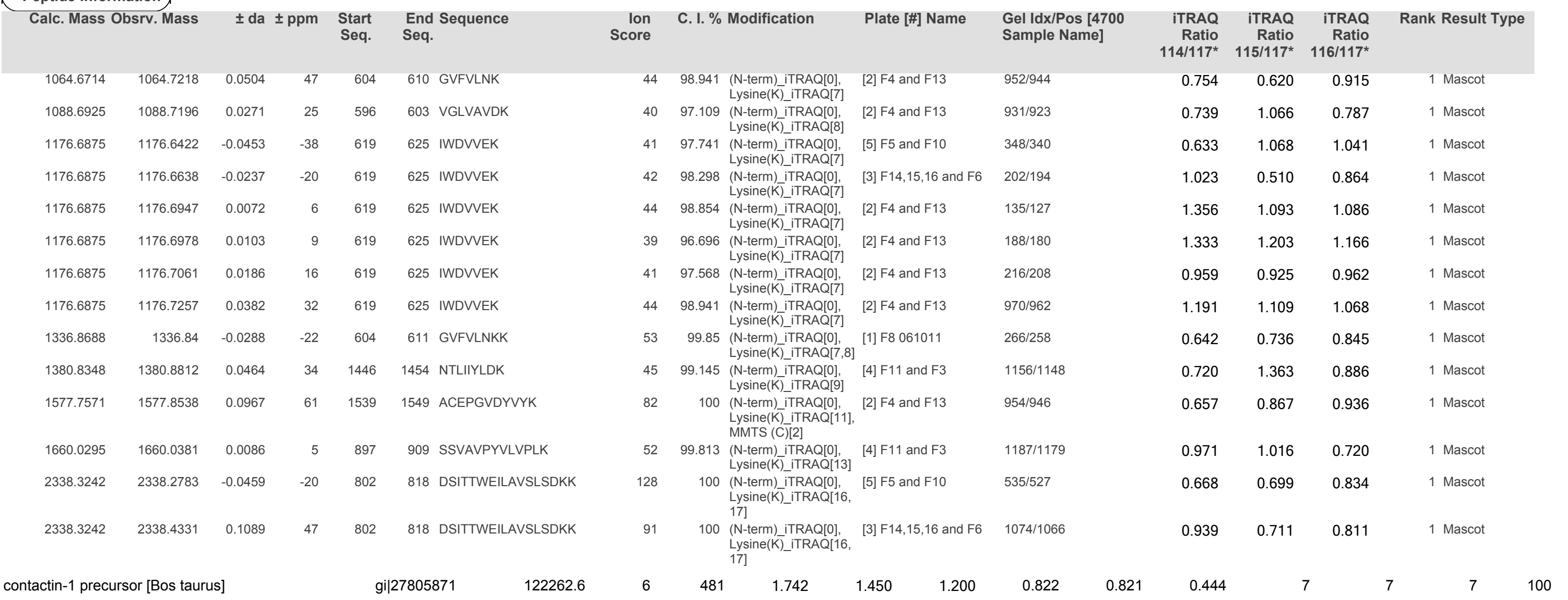

19 contactin-1 precursor [Bos taurus]

122262.

$\begin{array}{lllll}481 & 1.742 & 1.450 & 1.200 & 0.822\end{array}$

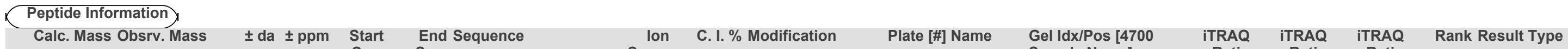

\begin{tabular}{|c|c|c|c|c|c|c|c|c|c|c|c|c|c|}
\hline \multicolumn{2}{|c|}{ Calc. Mass Obsrv. Mass } & \pm da & ppm & $\begin{array}{l}\text { Start } \\
\text { Seq. }\end{array}$ & $\begin{array}{l}\text { End Sequence } \\
\text { Seq. }\end{array}$ & $\begin{array}{l}\text { lon } \\
\text { Score }\end{array}$ & C. I. \% Modification & Plate [\#] Name & $\begin{array}{l}\text { Gel Idx/Pos [4700 } \\
\text { Sample Name] }\end{array}$ & $\begin{array}{r}\text { iTRAQ } \\
\text { Ratio }\end{array}$ & $\begin{array}{r}\text { iTRAQ } \\
\text { Ratio }\end{array}$ & $\begin{array}{r}\text { iTRAQ } \\
\text { Ratio }\end{array}$ & Rank Result Typ \\
\hline 1430.8127 & 1430.7853 & -0.0274 & -19 & 722 & 732 ELTITWAPLSR & 42 & 98.108 (N-term)_iTRAQ[0] & [8] F2 & 441/433 & 1.556 & 1.216 & 0.968 & 1 Mascot \\
\hline 1657.834 & 1657.7791 & -0.0549 & -33 & 654 & 667 TDPPIIEGNMEAAR & 56 & 99.923 (N-term)_iTRAQ[0] & [8] F2 & 293/285 & 1.492 & 1.166 & 1.091 & 1 Mascot \\
\hline 1829.9293 & 1829.8489 & -0.0804 & -44 & 844 & 856 YWASHDKEAAAHR & 57 & 99.939 (N-term) iTRAQ[0], & [3] F14,15,16 and F6 & 123/115 & 1.163 & 0.744 & 0.765 & 1 Mascot \\
\hline 1999.9553 & 1999.8577 & -0.0976 & -49 & 701 & 721 TDGAAPNVAPSDVGGGG & 124 & 100 (N-term)_iTRAQ[0] & [8] F2 & 196/188 & 4.003 & 3.390 & 1.999 & 1 Mascot \\
\hline 2039.1913 & 2039.2516 & 0.0603 & 30 & 236 & 249 TTKPYPADIVVQFK & 79 & 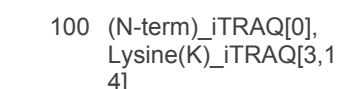 & [3] F14,15,16 and F6 & $918 / 910$ & 1.228 & 1.135 & 0.945 & 1 Mascot \\
\hline 2385.2368 & 2385.1453 & -0.0915 & -38 & 699 & 721 IKTDGAAPNVAPSDVGG & 42 & 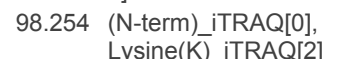 & [5] F5 and F10 & $226 / 218$ & 2.079 & 1.841 & 1.502 & 1 Mascot \\
\hline 2385.2368 & 2385.2695 & 0.0327 & 14 & 699 & 721 IKTDGAAPNVAPSDVGG & 124 & 100 (N-term) iTRAQ[0], & [3] F14,15,16 and F6 & $756 / 748$ & 1.765 & 1.802 & 1.559 & 1 Mascot \\
\hline
\end{tabular}

20 transthyretin precursor [Ovis aries

gi|57526651 17582.2

\begin{tabular}{|c|c|c|c|c|c|c|c|c|c|c|c|c|c|c|}
\hline \multicolumn{12}{|c|}{ Peptide Information } & & & \\
\hline Calc. Mass $\mathrm{C}$ & srv. Mass & \pm da & $\pm \mathrm{ppm}$ & $\begin{array}{l}\text { Start } \\
\text { Seq. }\end{array}$ & $\begin{array}{l}\text { End Sequence } \\
\text { Seq. }\end{array}$ & $\begin{array}{l}\text { lon } \\
\text { Score }\end{array}$ & C. I. \% & Modification & Plate [\#] Name & $\begin{array}{l}\text { Gel Idx/Pos [4700 } \\
\text { Sample Name] }\end{array}$ & $\begin{array}{r}\text { iTAQQ } \\
\text { Ratio } \\
114 / 117^{*}\end{array}$ & $\begin{array}{r}\text { iTAQQ } \\
\text { Ratio } \\
115 / 117^{*}\end{array}$ & $\begin{array}{r}\text { iTRAQ } \\
\text { Ratio } \\
116 / 117^{*}\end{array}$ & Rank Result Type \\
\hline 1187.6993 & 1187.6165 & -0.0828 & -70 & 42 & 51 GSPAANVGVK & 51 & 99.796 & 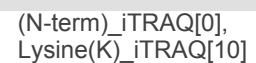 & [5] F5 and F10 & 153/145 & 0.362 & 0.774 & 1.345 & 1 Mascot \\
\hline 1187.6993 & 1187.7024 & 0.0031 & 3 & 42 & 51 GSPAANVGVK & 71 & 99.998 & 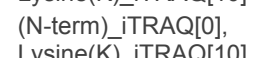 & [2] F4 and F13 & $164 / 156$ & 0.599 & 0.761 & 0.990 & 1 Mascot \\
\hline 1696.8428 & 1696.8508 & 0.008 & 5 & 56 & 68 AADETWEPFASGK & 52 & 99.824 & (N-term) iTRAQ[0], & [2] F4 and F13 & $136 / 128$ & 0.823 & 0.909 & 1.062 & 1 Mascot \\
\hline 1696.8428 & 1696.8597 & 0.0169 & 10 & 56 & 68 AADETWEPFASGK & 50 & 99.682 & (N-term) iTRAQ[0] & [2] F4 and F13 & 203/195 & 0.939 & 0.739 & 1.634 & 1 Mascot \\
\hline 1696.8428 & 1696.8615 & 0.0187 & 11 & 56 & 68 AADETWEPFASGK & 83 & 100 & 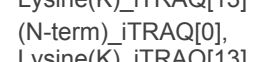 & [2] F4 and F13 & 188/180 & 0.806 & 1.074 & 1.094 & 1 Mascot \\
\hline
\end{tabular}




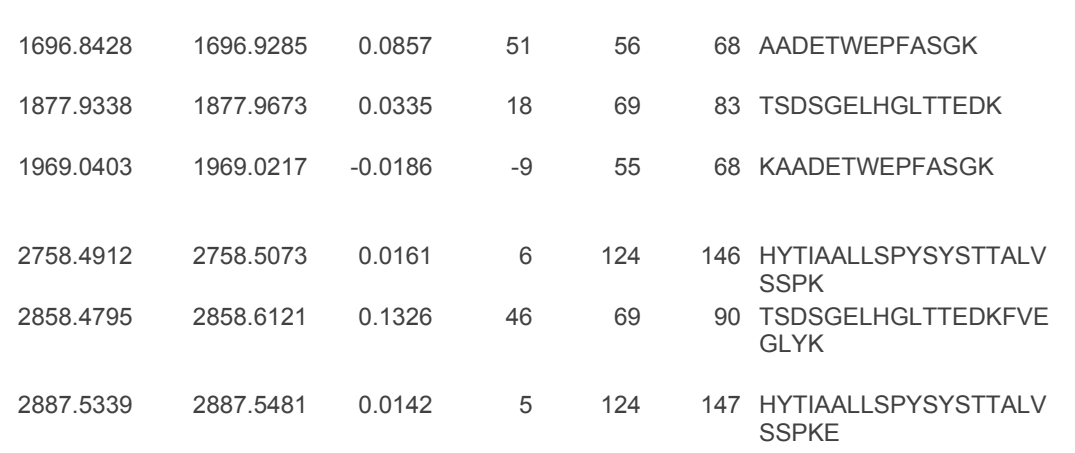

\begin{tabular}{|c|c|c|c|c|c|c|c|}
\hline 72 & 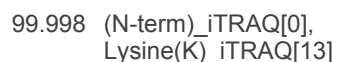 & [2] F4 and F13 & $963 / 955$ & 0.671 & 0.720 & 1.017 & 1 Mascot \\
\hline 101 & 100 (N-term) iTRAQ[0], & [3] F14,15,16 and F6 & $759 / 751$ & 0.585 & 0.626 & 0.945 & 1 Mascot \\
\hline 54 & 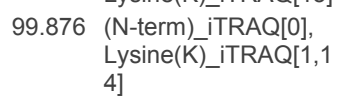 & [1] F8 061011 & 303/295 & 0.470 & 1.012 & 1.555 & 1 Mascot \\
\hline 46 & 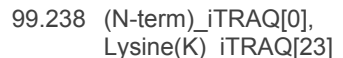 & [2] F4 and F13 & $364 / 356$ & 1.156 & 1.129 & 1.323 & 1 Mascot \\
\hline 62 & 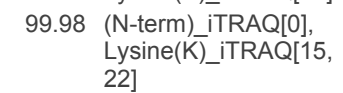 & [5] F5 and F10 & $1260 / 1252$ & 0.266 & 0.162 & 0.675 & 1 Mascot \\
\hline 49 & 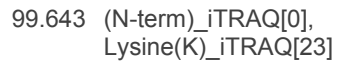 & [2] F4 and F13 & $364 / 356$ & 0.779 & 0.867 & 0.728 & 1 Mascot \\
\hline
\end{tabular}

21 complement component $\mathrm{C} 4$ [Ovis aries] gi|1227

15519.9

$\begin{array}{lllll}463 & 0.840 & 0.957 & 1.063 & 0.351\end{array}$

\begin{tabular}{|c|c|c|c|c|c|}
\hline \multicolumn{2}{|c|}{ Calc. Mass Obsrv. Mass } & \multicolumn{2}{|c|}{ \pm da $\pm \mathrm{ppm}$} & $\begin{array}{l}\text { Start } \\
\text { Seq. }\end{array}$ & $\begin{array}{l}\text { End Sequence } \\
\text { Seq. }\end{array}$ \\
\hline 1074.6769 & 1074.6154 & -0.0615 & -57 & 38 & 44 AVDLIQK \\
\hline 1305.646 & 1305.6111 & -0.0349 & -27 & 56 & 65 DGSYGAWLHR \\
\hline 1655.9254 & 1655.9398 & 0.0144 & 9 & 66 & 77 DSSTWLTAFVLK \\
\hline 1717.9358 & 1717.9036 & -0.0322 & -19 & 93 & 105 LQETATWLLSQQR \\
\hline 1778.7928 & 1778.7198 & -0.073 & -41 & 106 & 119 DDGSFHDPFPVMDR \\
\hline 1830.0219 & 1830.042 & 0.0201 & 11 & 78 & 92 ILSLAQDQVGGSPEK \\
\hline $2380.3135 \mathrm{H}$ & 2380.197 & -0.1165 - & -49 & 20 & 35 YLDKTEQWSLLPPETt \\
\hline
\end{tabular}

$44 \quad 98.817$ (N-term) $[$ iTRAQ[0], $\quad[5]$ F5 and F10

Gel Idx/Pos [4700

Rank Result Type

$\begin{array}{rrr}0.525 & 0.750 & 0.895\end{array}$

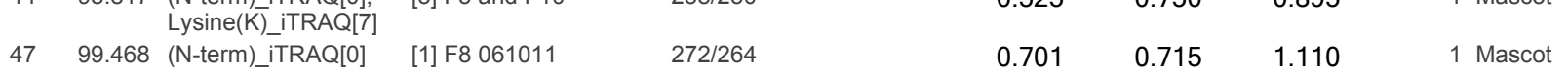

6399.986 (N-term) $B$ TRAQ[0], [4] F11 and F3 $\quad 127911271$

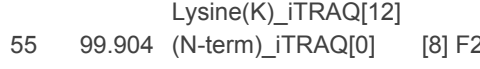

99.989 (N- $943 / 435$

$\begin{array}{llll}0.631 & 0.754 & 0.755 & 1 \text { Mascot }\end{array}$

$96 \quad 100$ (N-term) iTRAQ[0], [4] F11 and F3 $\quad{ }_{1094 / 1086}$

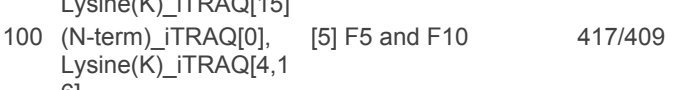

$\begin{array}{llll}1.664 & 1.525 & 1.310 \quad 1 \text { Mascot }\end{array}$

$\begin{array}{llll}0.826 & 0.829 & 0.989 & 1 \text { Mascot }\end{array}$

$\begin{array}{llll}1.047 & 1.360 & 1.205 & 1 \text { Mascot }\end{array}$

$\begin{array}{llllll}2380.3135 & 2380.197 & -0.1165 & -49 & 20 & 35 \\ \text { YLDKTEQWSLLPPETK }\end{array}$

RecName: Full=Complement C4; Contains: RecName:
Full=Complement C4 alpha chain; Contains: RecName:

Peptide Information

\begin{tabular}{|c|c|c|c|c|c|c|c|c|c|c|c|c|c|c|}
\hline \multicolumn{2}{|c|}{ Calc. Mass Obsrv. Mass } & \multicolumn{2}{|c|}{ \pm da $\pm p p m$} & \multirow{2}{*}{$\begin{array}{l}\text { Start } \\
\text { Seq. } \\
\\
226\end{array}$} & \multirow{2}{*}{$\begin{array}{l}\text { End Sequence } \\
\text { Seq. } \\
232 \text { AVDLIQK }\end{array}$} & \multirow{2}{*}{$\begin{array}{r}\begin{array}{r}\text { Ion } \\
\text { Score }\end{array} \\
44\end{array}$} & \multicolumn{2}{|c|}{ C. I. \% Modification } & \multirow{2}{*}{$\begin{array}{l}\text { Plate [\#] Name } \\
\text { [5] F5 and F10 }\end{array}$} & $\begin{array}{l}\text { Gel Id dx/Pos [4700 } \\
\text { Sample Name] }\end{array}$ & \multirow{2}{*}{$\begin{array}{r}\text { iTRAQ } \\
\text { Ratio } \\
114 / 117^{*} \\
0.525\end{array}$} & \multirow{2}{*}{$\begin{array}{r}\text { iTRAQ } \\
\text { Ratio } \\
115 / 117^{*} \\
0.750\end{array}$} & \multirow{2}{*}{$\begin{array}{r}\text { iTRAQ } \\
\text { Ratio } \\
116 / 117^{*} \\
0.895\end{array}$} & Rank Result Type \\
\hline 1074.6769 & 1074.6154 & -0.0615 & -57 & & & & 98.817 & $(\mathrm{~N}$-term)_iTRAQ[0], & & $258 / 250$ & & & & 1 Mascot \\
\hline 1109.6565 & 1109.5997 & -0.0568 & -51 & 351 & 358 ANTFLGAK & 55 & 99.919 & 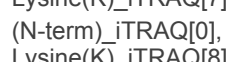 & [5] F5 and F10 & $247 / 239$ & 0.998 & 1.027 & 0.949 & 1 Mascot \\
\hline 1305.646 & 1305.6111 & -0.0349 & -27 & 244 & 253 DGSYGAWLHR & 47 & 99.468 & (N-term) iTRAQ[0] & [1] F8 061011 & $272 / 264$ & 0.701 & 0.715 & 1.110 & 1 Mascot \\
\hline 1351.7831 & 1351.8199 & 0.0368 & 27 & 12 & 21 LGQYTSPVAK & 41 & 97.714 & (N-term) iTRAQ[0], & [2] F4 and F13 & $921 / 913$ & 1.681 & 1.345 & 1.936 & 1 Mascot \\
\hline 1374.8354 & 1374.892 & 0.0566 & 41 & 823 & 831 ITQVLHFTK & 64 & 99.988 & (N-term) iTRAQ[0], & [7] F12 and F9 and F7 & $1346 / 1338$ & 1.146 & 1.333 & 1.118 & 1 Mascot \\
\hline 1655.9254 & 1655.9398 & 0.0144 & 9 & 254 & 265 DSSTWLTAFVLK & 63 & 99.986 & (N-term) iTRAQ[0], & [4] F11 and F3 & 1279/1271 & 0.631 & 0.754 & 0.755 & 1 Mascot \\
\hline 1667.9102 & 1667.9253 & 0.0151 & 9 & 530 & 541 LEEELQFSLGSK & 47 & 99.482 & (N-term) iTRAQ[0], & [4] F11 and F3 & $1166 / 1158$ & 0.823 & 0.922 & 1.014 & 1 Mascot \\
\hline 1712.9429 & 1712.8804 & -0.0625 & -36 & 517 & 529 SHVLQLTNHQVHR & 44 & 98.967 & (N-term)_iTRAQ[0] & [3] F14,15,16 and F6 & $133 / 125$ & 0.926 & 1.225 & 1.275 & 1 Mascot \\
\hline 1717.9358 & 1717.9036 & -0.0322 & -19 & 281 & 293 LQETATWLLSQQR & 55 & 99.904 & (N-term)_iTRAQ[0] & {$[8] F 2$} & 443/435 & 1.664 & 1.525 & 1.310 & 1 Mascot \\
\hline & & & & & 5586 & 7 & 455 & 0.807 & 0.930 & 0.314 & 0.180 & & 9 & 9 \\
\hline
\end{tabular}

23 clusterin preproprotein [Bos taurus]

gi|27806007

\begin{tabular}{|c|c|c|c|c|c|}
\hline \multirow{2}{*}{\multicolumn{2}{|c|}{$\begin{array}{l}\text { Peptide Information } \\
\text { Calc. Mass Obsrv. Mass }\end{array}$}} & \multirow{2}{*}{\multicolumn{2}{|c|}{ \pm da \pm ppm }} & \multirow{2}{*}{$\begin{array}{l}\text { Start } \\
\text { Seq. }\end{array}$} & \multirow{2}{*}{$\begin{array}{l}\text { End Sequence } \\
\text { Seq. }\end{array}$} \\
\hline & & & & & \\
\hline 1197.6877 _ & 1197.6389 & -0.0488 & -41 & 209 & 216 GSLFFNPK \\
\hline 1197.6877 & 1197.7462 & 0.0585 & 49 & 209 & 216 GSLFFNPK \\
\hline 1390.8403 & 1390.8541 & 0.0138 & 10 & 63 & 72 LLLSSLEEEAK \\
\hline 1555.7546 & 1555.7681 & 0.0135 & 9 & 177 & 188 ASSIMDELFQDR \\
\hline 1555.7546 & 1555.7887 & 0.0341 & 22 & 177 & 188 ASSIMDELFQDR \\
\hline 1663.0377 & 1663.2009 & 0.1632 & 98 & 62 & 72 KLLLSSLEEAK \\
\hline 1814.9899 & 1815.0369 & 0.047 & 26 & 332 & 343 LYDQLLQSYQQK \\
\hline
\end{tabular}

Plate [\#] Name Gel Idx/Pos [4700 iTRAQ ITRAQ iTRAQ Rank Result Type

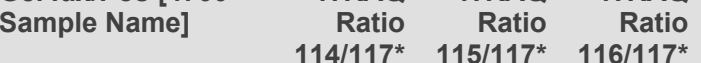

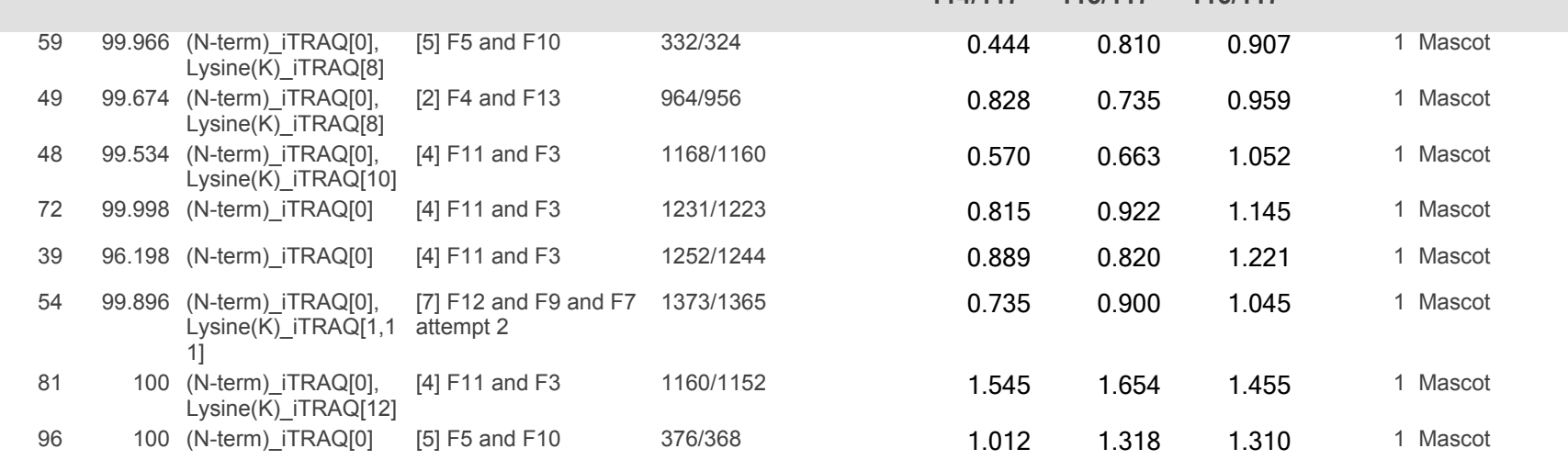


2120.0322

\section{9.}

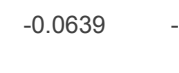

193

208 RPQDTQYYSPFSSFPR

$46 \quad 99.234$ (N-term)_iTRAQ[0] [5] F5 and F10 3844/376

0.837

24 albumin [Sus scrofa]

gil164318

79319.3

$\begin{array}{llll}7 & 442 \quad 0.940 \quad 1069 \quad 0.024 & 1048\end{array}$

0.837

$0.894 \quad 0.940$

1 Mascot

Protein Group

gil $833798 \quad 79333.3$

serum albumin precursor [Sus scrofa]

gi|52353352 $\quad 79758.6$

\begin{tabular}{|c|c|c|c|c|c|}
\hline \multicolumn{2}{|c|}{ Calc. Mass Obsrv. Mass } & \multicolumn{2}{|c|}{ \pm da $\pm \mathrm{ppm}$} & $\begin{array}{l}\text { Start } \\
\text { Seq. }\end{array}$ & $\begin{array}{l}\text { End Sequence } \\
\text { Seq. }\end{array}$ \\
\hline 1175.6508 & 1175.6292 & -0.0216 & -18 & 481 & 487 LCVLHEK \\
\hline 1175.6508 & 1175.6975 & 0.0467 & 40 & 481 & 487 LCVLHEK \\
\hline 1260.5291 & 1260.5676 & 0.0385 & 31 & 497 & 505 CCTESLVNR \\
\hline 1302.8242 & 1302.7657 & -0.0585 & -45 & 547 & 555 QTALVELLK \\
\hline 1302.8242 & 1302.7715 & -0.0527 & -40 & 547 & 555 QTALVELLK \\
\hline 1302.8242 & 1302.8359 & 0.0117 & 9 & 547 & 555 QTALVELLK \\
\hline 1302.8242 & 1302.8368 & 0.0126 & 10 & 547 & 555 QTALVELLK \\
\hline 1302.8242 & 1302.8618 & 0.0376 & 29 & 547 & 555 QTALVELLK \\
\hline 1344.6519 & 1344.7135 & 0.0616 & 46 & 411 & 418 QNCELFEK \\
\hline 1344.6519 & 1344.7535 & 0.1016 & 76 & 411 & 418 QNCELFEK \\
\hline 1575.0216 & 1574.9834 & -0.0382 & -24 & 546 & 555 KQTALVELLK \\
\hline 1575.0216 & 1575.0242 & 0.0026 & 2 & 546 & 555 KQTALVELLK \\
\hline 1575.0216 & 1575.1365 & 0.1149 & 73 & 546 & 555 KQTALVELLK \\
\hline 1860.6581 & 1860.5664 & -0.0917 & -49 & 265 & 278 ECCHGDLLECADDR \\
\hline 1860.6581 & 1860.6014 & -0.0567 & -30 & 265 & 278 ECCHGDLLECADDR \\
\hline 1860.6581 & 1860.6028 & -0.0553 & -30 & 265 & 278 ECCHGDLLECADDR \\
\hline 1860.6581 & 1860.6277 & -0.0304 & -16 & 265 & 278 ECCHGDLLECADDR \\
\hline 1860.6581 & 1860.6558 & -0.0023 & -1 & 265 & 278 ECCHGDLLECADDR \\
\hline 1860.6581 & 1860.7362 & 0.0781 & 42 & 265 & 278 ECCHGDLLECADDR \\
\hline 1860.6581 & 1860.74 & 0.0819 & 44 & 265 & 278 ECCHGDLLECADDR \\
\hline 2503.0405 & 2502.9846 & -0.0559 & -22 & 265 & $\begin{array}{l}283 \text { ECCHGDLLECADDRADL } \\
\text { AK }\end{array}$ \\
\hline
\end{tabular}

25 fibronectin

gi|224863 $\quad 95322.7$ son

C. I. \% Modification Plate [\#] Name

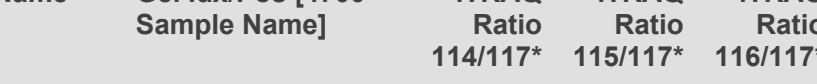

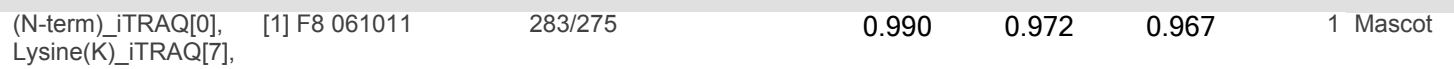

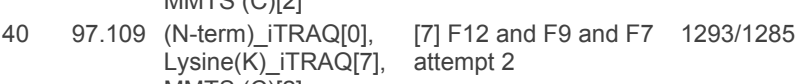

$43 \quad 98.581 \begin{aligned} & \text { (N-term) itRAQ[0], } \\ & \text { MMTS (C) [1, } 1,2]\end{aligned}$

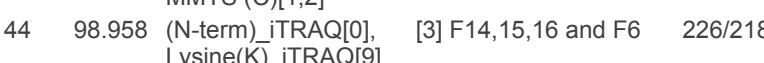

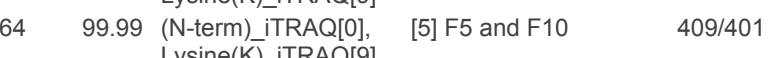

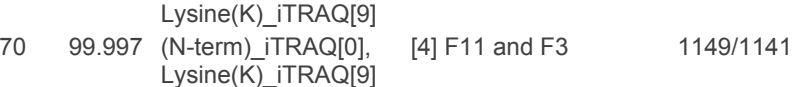

6499.99 (N-term) iTRAQ[0], [2] F4 and F13 235/227

$64 \quad 99.988$ (N-term) iTRAQ[0], [2] F4 and F13 995/987

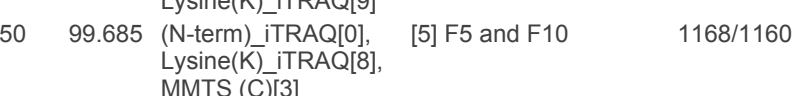

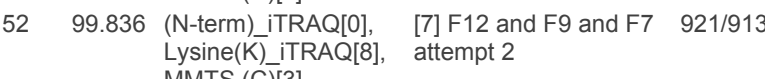

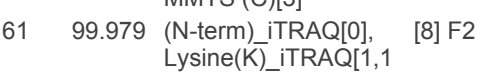

$375 / 367$

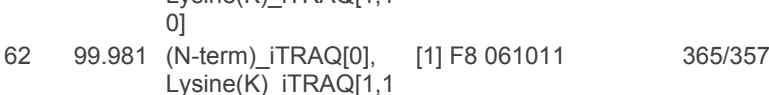

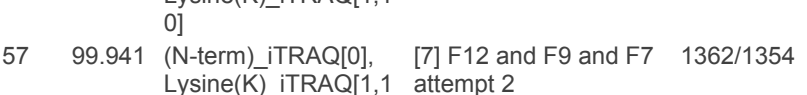

$92 \quad 100$ (N)-erm)iTRAQ[0], [5] F5 and F10 4744466

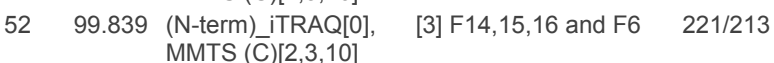

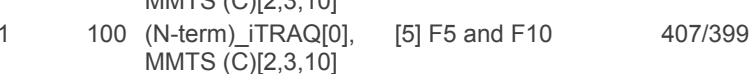

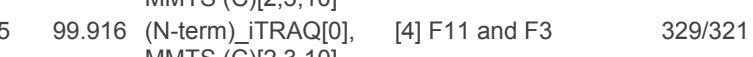

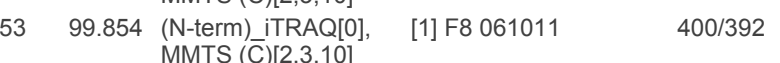

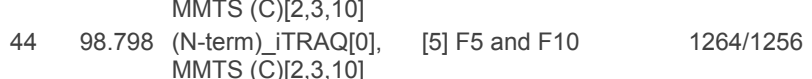

100 (N-term) ITRAQ[0], $\quad[3] \mathrm{F} 14,15,16$ and F6 $\quad 930 / 922$

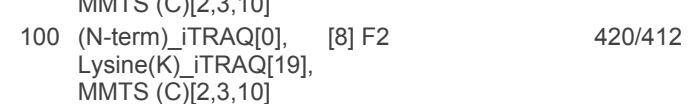

Lysine(K) $[$ iTRAQ[19],
MMTS (C) $[2,3,10]$

$\begin{array}{llll}1.301 & 1.047 & 0.748 & 1 \text { Mascot } \\ 1.992 & 2.408 & 1.293 & 1 \text { Mascot } \\ 1.249 & 2.368 & 1.376 & 1 \text { Mascot } \\ 1.135 & 1.403 & 0.860 & 1 \text { Mascot } \\ 1.196 & 1.443 & 0.987 & 1 \text { Mascot } \\ 0.663 & 1.379 & 1.131 & 1 \text { Mascot } \\ 0.751 & 1.218 & 1.051 & 1 \text { Mascot } \\ 0.100 & 0.100 & 0.184 & 1 \text { Mascot } \\ & & & 1 \text { Mascot } \\ 0.158 & 0.131 & 0.285 & \\ 1.065 & 1.281 & 1.002 & 1 \text { Mascot } \\ & & & \\ 0.570 & 1.183 & 1.125 & 1 \text { Mascot } \\ 0.912 & 0.993 & 0.968 & 1 \text { Mascot } \\ 1.263 & 1.107 & 0.804 & 1 \text { Mascot } \\ 0.796 & 1.173 & 0.796 & 1 \text { Mascot } \\ 1.372 & 1.168 & 1.163 & 1 \text { Mascot } \\ 3.021 & 1.621 & 1.794 & 1 \text { Mascot } \\ 1.452 & 1.453 & 1.660 & 1 \text { Mascot } \\ 1.608 & 2.083 & 1.057 & 1 \text { Mascot } \\ 1.021 & 1.161 & 1.116 & 1 \text { Mascot } \\ 1.456 & 1.205 & 0.971 & 1 \text { Mascot }\end{array}$

Peptide Information

\begin{tabular}{|c|c|c|c|c|c|c|c|c|c|c|c|c|c|c|}
\hline \multicolumn{2}{|c|}{ Calc. Mass Obsrv. Mass } & \multicolumn{2}{|c|}{ $\pm d a \pm p p m$} & \multirow{2}{*}{$\begin{array}{l}\text { Start } \\
\text { Seq. } \\
\\
296\end{array}$} & \multirow{2}{*}{$\begin{array}{l}\text { End Sequence } \\
\text { Seq. } \\
305 \text { FTNVGPDTMR }\end{array}$} & \multirow{2}{*}{$\begin{array}{r}\begin{array}{r}\text { lon } \\
\text { Score }\end{array} \\
\\
55\end{array}$} & \multirow{2}{*}{\multicolumn{2}{|c|}{$\begin{array}{l}\text { C. I. \% Modification } \\
\text { 99.917 (N-term)_iTRAQ[0] }\end{array}$}} & \multirow{2}{*}{$\begin{array}{l}\text { Plate [\#] Name } \\
{[4] \text { F11 and F3 }}\end{array}$} & \multirow{2}{*}{$\begin{array}{l}\text { Gel Idx/Pos [4700 } \\
\text { Sample Name] } \\
1019 / 1011\end{array}$} & \multirow{2}{*}{$\begin{array}{r}\text { iTRAQ } \\
\text { Ratio } \\
114 / 117^{*} \\
1.953\end{array}$} & \multirow{2}{*}{$\begin{array}{r}\text { iTRAQ } \\
\text { Ratio } \\
115 / 117^{*} \\
1.338\end{array}$} & \multirow{2}{*}{$\begin{array}{r}\text { iTRAQ } \\
\text { Ratio } \\
116 / 117^{*} \\
1.460\end{array}$} & \multirow{2}{*}{$\begin{array}{c}\text { Rank Result Type } \\
1 \text { Mascot }\end{array}$} \\
\hline 12816382 & 1281.6561 & 0.0179 & 14 & & & & & & & & & & & \\
\hline 1707.8938 & 1707.8341 & -0.0597 & -35 & 142 & 155 QYNVGPAASQYPLR & 58 & 99.958 & (N-term) iTRAQ[0] & [8] F2 & $301 / 293$ & 1.451 & 1.189 & 0.879 & 1 Mascot \\
\hline 1707.8938 & 1707.9291 & 0.0353 & 21 & 142 & 155 QYNVGPAASQYPLR & 49 & 99.659 & (N-term)_iTRAQ[0] & [4] F11 and F3 & 1040/1032 & 1.323 & 1.093 & 0.813 & 1 Mascot \\
\hline 1773.9731 & 1773.9127 & -0.0604 & -34 & 40 & 54 VDVIPVNLPGEHGQR & 51 & 99.767 & (N-term)_iTRAQ[0] & [5] F5 and F10 & 346/338 & 0.674 & 0.657 & 0.731 & 1 Mascot \\
\hline 1865.9967 & 1866.0474 & 0.0507 & 27 & 546 & 560 GDSPASSKPVSINYR & 52 & 99.828 & 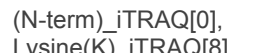 & {$[3] F 14,15,16$ and $F 6$} & $760 / 752$ & 0.829 & 0.498 & 0.774 & 1 Mascot \\
\hline 1963.111 & 1963.114 & 0.003 & 2 & 156 & 171 NLQPGSEYAVSLVAVK & 67 & 99.994 & (N-term) iTRAC & [4] F11 and F3 & $1137 / 1129$ & 2.700 & 2.812 & 1.632 & 1 Mascot \\
\hline 2405.3208 & 2405.2563 & -0.0645 & -27 & 616 & $\begin{array}{l}636 \underset{\text { ATK }}{\text { EINLAPDSSSVVVSGLMV }} \\
\text { ATK }\end{array}$ & 138 & 100 & $\begin{array}{l}\text { (N-term) iTRAQ[0], } \\
\text { Lysine(K)_iTRAQ[21] }\end{array}$ & {$[8] \mathrm{F} 2$} & $472 / 464$ & 1.162 & 0.630 & 1.119 & 1 Mascot \\
\hline
\end{tabular}


26 keratin, type I cytoskeletal 9 [Homo sapiens] cytokeratin 9 [Homo sapiens]

cytokeratin 9 [Homo sapiens]

keratin 9 [Homo sapiens]

Peptide Information

Calc. Mass Obsrv. Mas

gi|55956899

66101.5

gi| 435476

gil 453155

66166.6

66024.5 $\begin{array}{lllllll}2849.2634 & 2849.2075 & -0.0559 & -20 & 64 & 95 \text { GGGGSFGYSYGGGSGG }\end{array}$

$\begin{array}{llllll}3511.4863 & 3511.269 & -0.2173 & -62 & 580 & \begin{array}{r}619 \\ \text { SGGSGSHHGGSGGFGE } \\ \text { SGSYGGGEASGSGG }\end{array}\end{array}$

immunoglobulin gamma-1 chain [Ovis aries]

Protein Group

Peptide Information
Ion C. $1 . \%$ Modification Plate [\#] Name

$53 \quad 99.847$ (

100 (Nsine(K)_iTRAQ[7,8] attempt 2

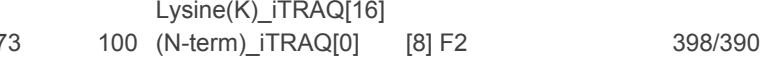

$\left.89 \quad 100 \begin{array}{c}(\text { N-term) iTRAQ[0], } \\ \text { Lysine(K)_iTRAQ[400] }\end{array}\right]$ [5] F5 and F10 203/195
Gel Idx/Pos $[4700$ iTRAQ iTRAQ iTRAQ Rank Result Type $\begin{array}{rrr}\text { Ratio } & \text { Ratio } & \text { Ratio } \\ 114 / 117^{*} & 115 / 117^{*} & 116 / 117^{*}\end{array}$

$\begin{array}{rrrr}4.530 & 6.290 & 2.241 \quad 1 \text { Mascot }\end{array}$

$\begin{array}{llll}1.525 & 2.142 & 1.027 & 1 \text { Mascot }\end{array}$

$\begin{array}{llll}2.238 & 3.619 & 1.791 & 1 \text { Mascot }\end{array}$

$\begin{array}{llll}1.187 & 1.147 & 0.908 & 1 \text { Mascot }\end{array}$

$\begin{array}{llllllllll}399 & 1.121 & 0.908 & 0.914 & 0.413 & 0.297 & 0.153 & 14 & 14 & 14\end{array}$

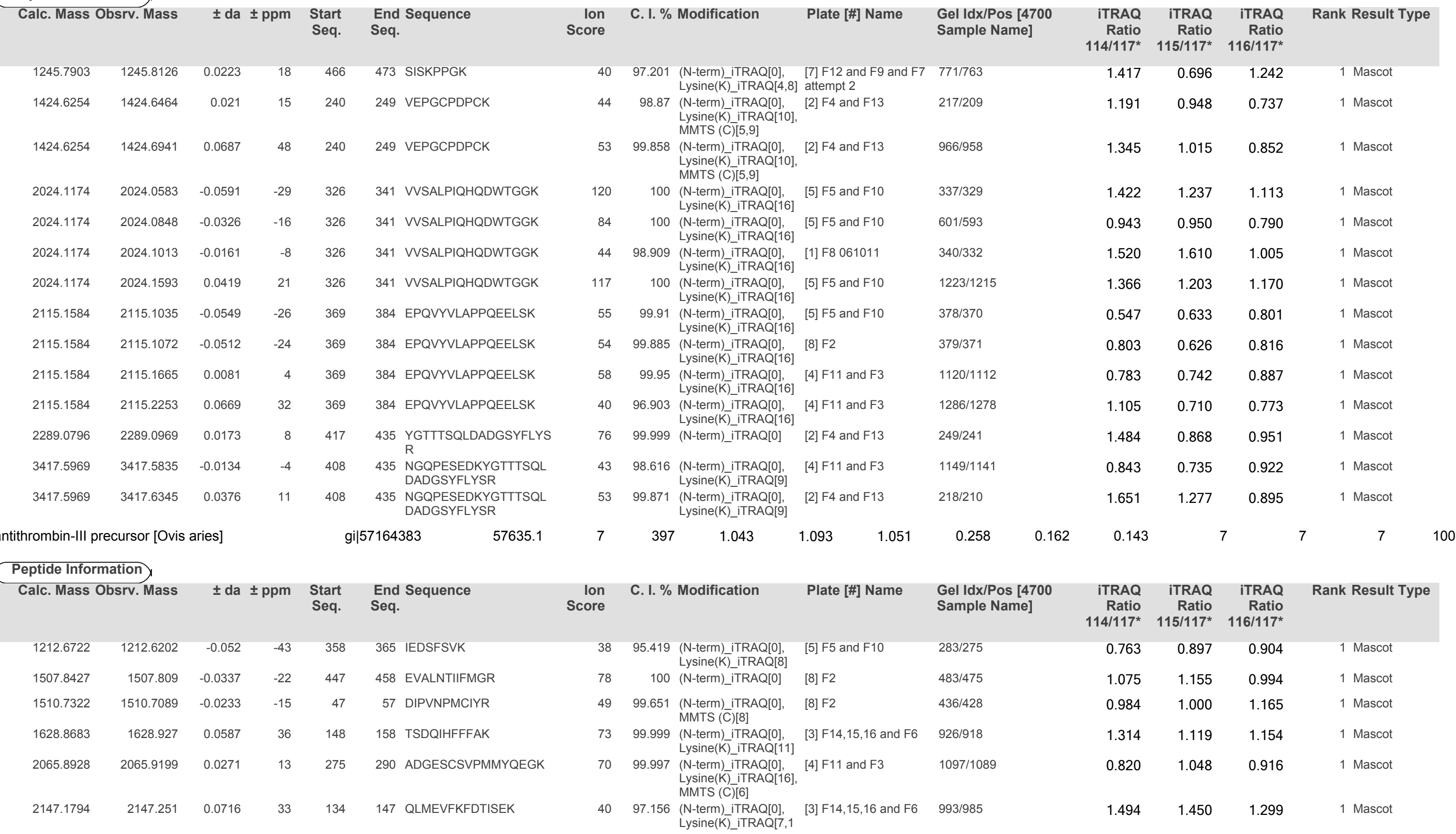

28 antithrombin-III precursor [Ovis aries]

$$
\text { Peptide Information }
$$

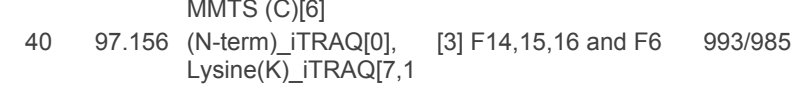

$\begin{array}{llll}1.494 & 1.450 & 1.299 & 1 \text { Mascot }\end{array}$ 
$\begin{array}{llllll}2586.3484 & 2586.3784 & 0.03 & 12 & 104 & 124 \\ \text { NKN NNDIFLSPLSISTAFAM }\end{array}$

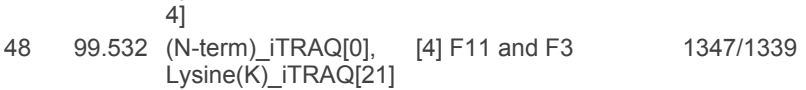

$\begin{array}{llll}1.031 & 1.059 & 0.985 & 1 \text { Mascot }\end{array}$

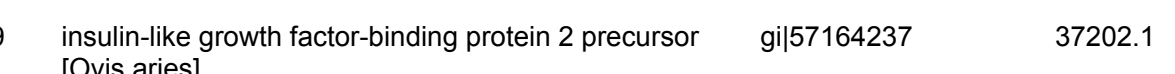

28

insulin-like growth factor-binding protein 2 precursor $\quad$ gi|27807005 $\quad 37028$

[Bos taurus]

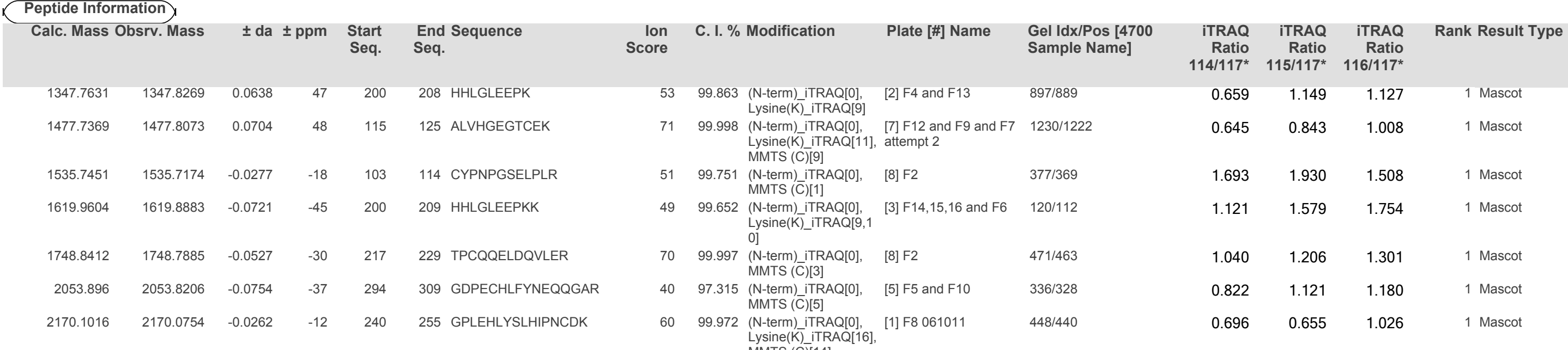

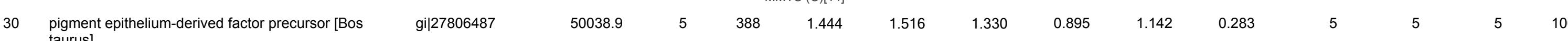

Peptide Information

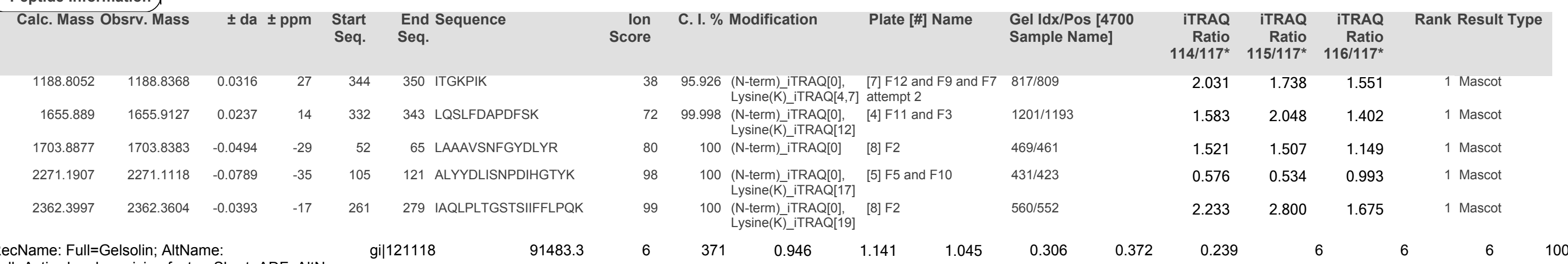

31 RecName: Full=Gelsolin; AltName: $\quad$ gil121118 $\quad 91483.3$ Full=Actin-depoly
Full=Brevin;

Peptide Information

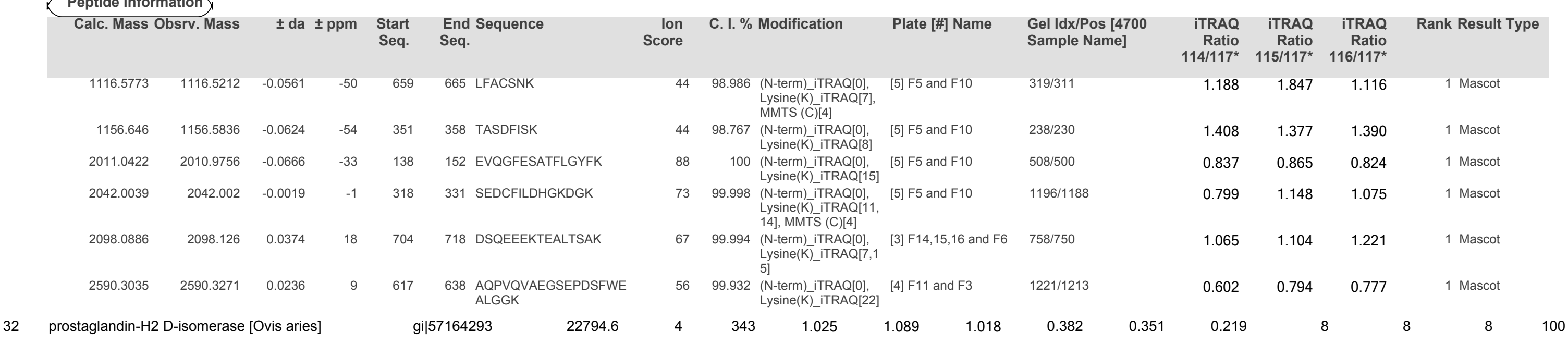

Peptide Information

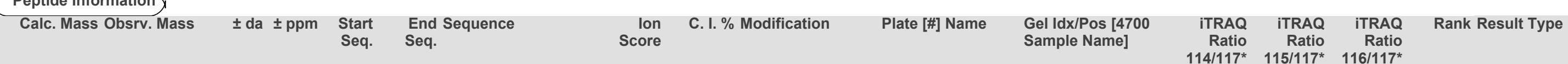

$\begin{array}{lllllllllllll}16088176 & 1608.7279 & -0.0897 & -56 & 138 & 151\end{array}$ 


$\begin{array}{rrrrrl}1608.8176 & 1608.781 & -0.0366 & -23 & 138 & 151 \text { GPGPDSLMATLYSR } \\ 1608.8176 & 1608.8326 & 0.015 & 9 & 138 & 151 \text { GPGPDSLMATLYSR } \\ 1825.0356 & 1825.0129 & -0.0227 & -12 & 169 & 182 \text { SLGFTEEGIVFLPK } \\ 1825.0356 & 1825.0688 & 0.0332 & 18 & 169 & 182 \text { SLGFTEEGIVFLPK } \\ 1825.0356 & 1825.0907 & 0.0551 & 30 & 169 & 182 \text { SLGFTEEGIVFLPK } \\ 2148.1011 & 2148.1353 & 0.0342 & 16 & 43 & 58 \text { WFTSGLASNSSWFLEK } \\ 2313.3076 & 2313.2129 & -0.0947 & -41 & 169 & 185 \text { SLGFTEEGIVFLPKTDK }\end{array}$

\begin{tabular}{|c|c|c|c|c|c|c|c|}
\hline 100 & (N-term)_iTRAQ[0] & [8] F2 & 420/412 & 0.812 & 0.730 & 0.887 & 1 Mascot \\
\hline 100 & (N-term)_iTRAQ[0] & [4] F11 and F3 & $1172 / 1164$ & 0.651 & 0.740 & 0.913 & 1 Mascot \\
\hline 95.615 & $\begin{array}{l}(\mathrm{N} \text {-term)iTRAQ[0], } \\
\text { Lysine(KK) iTRAQ[14] }\end{array}$ & [5] F5 and F10 & $1356 / 1348$ & 1.538 & 1.409 & 1.558 & 1 Mascot \\
\hline 100 & $\begin{array}{l}\text { (N-term) ITRAQ[0]] } \\
\text { Lyine(KK) }\end{array}$ & [4] F11 and F3 & $1247 / 1239$ & 0.999 & 0.863 & 1.011 & 1 Mascot \\
\hline 99.992 & $\begin{array}{l}\text { (N-term) iTRACIO] } \\
\text { Lysine(KK) iTRAQ[14] }\end{array}$ & [2] F4 and F13 & $325 / 317$ & 0.802 & 1.288 & 0.873 & 1 Mascot \\
\hline 97.471 & (N-term) TTRAQ[0], & [4] F11 and F3 & 1299/1291 & 1.796 & 1.576 & 1.025 & 1 Mascot \\
\hline 100 & $\begin{array}{l}\text { (N-term) TTRAQ[0], } \\
\text { Lysine(K)_iTRAQ[14, }\end{array}$ & [5] F5 and F10 & 442/434 & 1.046 & 1.266 & 1.207 & 1 Mascot \\
\hline
\end{tabular}

33 beta-2-microglobulin precursor [Ovis aries]

gi|57164311 $\quad 14844.8$

Peptide Information

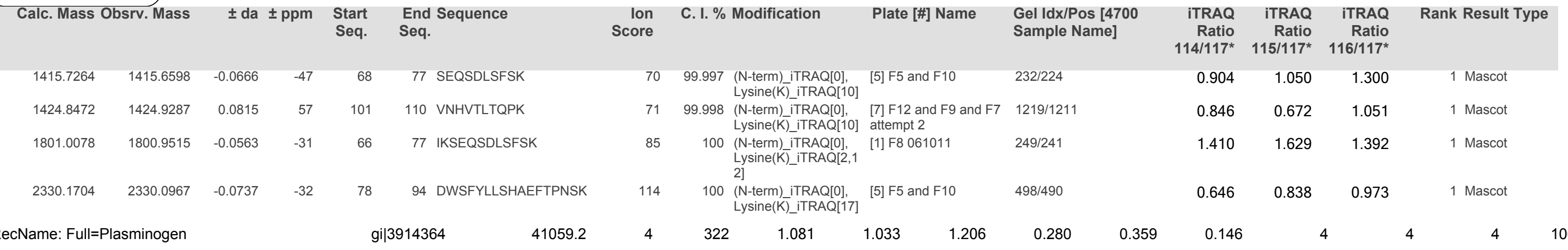

34 RecName: Full=Plasminogen

\begin{tabular}{|c|c|c|c|c|c|c|c|c|c|c|c|c|c|}
\hline Calc. Mass C & siv. Mass & \pm da & ppm & $\begin{array}{l}\text { Start } \\
\text { Seq. }\end{array}$ & $\begin{array}{l}\text { End Sequence } \\
\text { Seq. }\end{array}$ & $\begin{array}{l}\text { lon } \\
\text { Score }\end{array}$ & C. I. \% Modification & Plate [\#] Name & $\begin{array}{l}\text { Gel Idx/Pos [4700 } \\
\text { Sample Name] }\end{array}$ & $\begin{array}{r}\text { iTRAQ } \\
\text { Ratio } \\
114 / 117^{*}\end{array}$ & $\begin{array}{r}\text { iTRAQ } \\
\text { Ratio } \\
115 / 117^{*}\end{array}$ & $\begin{array}{r}\text { iTRAQ } \\
\text { Ratio } \\
116 / 117^{*}\end{array}$ & Rank Result Type \\
\hline 1358.74 & 1358.7659 & 0.0259 & 19 & 179 & 189 EASVQEIPVSR & 59 & 99.967 (N-term)_iTRAQ[0] & [4] F11 and F3 & 990/982 & 1.094 & 1.065 & 1.428 & 1 Mascot \\
\hline 1754.8907 & 1754.85 & -0.0407 & -23 & 329 & 341 VSTYVPWIEETMR & 49 & 99.67 (N-term)_iTRAQ[0] & [8] F2 & $485 / 477$ & 1.339 & 1.485 & 1.250 & 1 Mascot \\
\hline 2095.9067 & 2095.8589 & -0.0478 & -23 & 67 & 83 NPDGDVNGPWCYTTNP & 76 & 99.999 (N-term) iTRAQ[0], & [8] F2 & $363 / 355$ & 1.260 & 1.111 & 1.064 & 1 Mascot \\
\hline 3243.3635 & 3243.3074 & -0.0561 & -17 & 274 & $\begin{array}{l}302 \begin{array}{l}\text { STELCAGDLAGGTDSCQ } \\
\text { GDSGGPLVCFEK }\end{array}\end{array}$ & 138 & 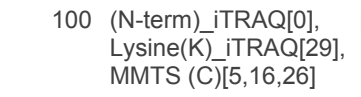 & [8] F2 & $518 / 510$ & 0.741 & 0.648 & 1.113 & 1 Mascot \\
\hline
\end{tabular}

ectonucleotide pyrophosphatase/phosphodiesterase
family member 2 2 Pongo abelii] $316-1.151-1.036$

Protein Group

Ectonucleotide pyrophosphatase/phosphodiesterase 2 gi|22137786 108526.8
[Homo sapiens]

$\begin{array}{lll}\text { autotaxin-t [Homo sapiens] } & \text { gil11160616 } & 108540.9\end{array}$

\begin{tabular}{|ccccrl}
$\begin{array}{c}\text { Peptide Information } \\
\text { Calc. Mass Obsrv. Mass }\end{array}$ & \pm da \pm ppm & $\begin{array}{l}\text { Start } \\
\text { Seq. }\end{array}$ & $\begin{array}{l}\text { End Sequence } \\
\text { Seq. }\end{array}$ \\
\hline 1622.8121 & 1622.7762 & -0.0359 & -22 & 314 & 326 YGPFGPEMTNPLR \\
1636.8456 & 1636.7698 & -0.0758 & -46 & 850 & 861 DIEHLTSLDFFR \\
1700.6986 & 1700.6548 & -0.0438 & -26 & 59 & 71 CFELQEAGPPDCR \\
1748.7751 & 1748.8528 & 0.0777 & 44 & 465 & 476 CFFQGDHGFDNK \\
1977.1782 & 1977.1207 & -0.0575 & -29 & 412 & 422 KPDQHFKPYLK \\
2295.1804 & 2295.137 & -0.0434 & -19 & 715 & 731 YDAFLVTNMVPMYPAFK \\
2437.2358 & 2437.2441 & 0.0083 & 3 & 696 & 714 QMSYGFLFPYLSSSPE \\
\hline
\end{tabular}

C. I. \% Modification Plate [\#] Name

Gel Idx/Pos [4700 iTRAQ iTRAQ iTRAQ Rank Result Type

$40 \quad 97.096$ (N-term)_iTRAQ[0] [8] F2 $\begin{array}{rrr}\text { Ratio } & \text { Ratio } & \text { Ratio } \\ 114 / 117^{*} & 115 / 117^{*} & 116 / 117^{*}\end{array}$

$\begin{array}{lllll}1.399 & 1.230 & 1.244 & 1 \text { Mascot }\end{array}$

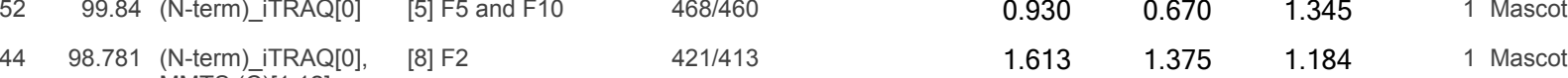

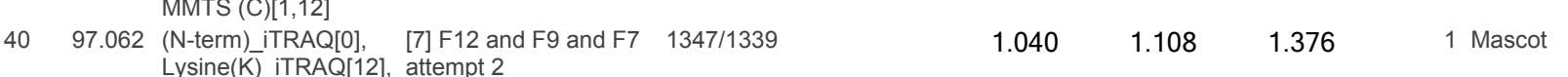

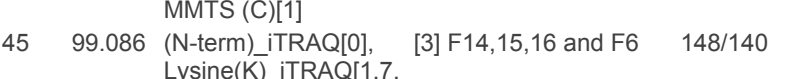

$49 \quad 99.667 \begin{aligned} & 11] \\ & (N-\text {-term) iTRAQ[0], } \\ & \text { Lysine(K) iTRAQ[1] }\end{aligned}$ [8] F2 $\quad 551 / 543$

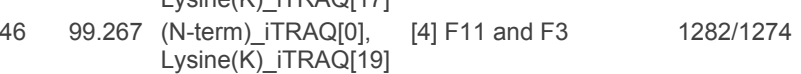

$\begin{array}{llll}0.704 & 0.780 & 0.995 & 1 \text { Mascot }\end{array}$

$\begin{array}{llll}2.164 & 2.163 & 1.722 & 1 \text { Mascot } \\ 0.805 & 0.604 & 0.665 & 1 \text { Mascot }\end{array}$

36 complement component $\mathrm{C} 4$ [Ovis aries]

gi|1235

15731.1

$\begin{array}{llllll}287 & 0.787 & 0.909 & 1.057 & 0.337 & 0.284\end{array}$

0.263

5

$5-100$

Peptide Information

Calc. Mass Obsrv. Mass \pm da \pm ppm Start $\quad$ End Sequence Ion $\quad$ C. I. \% Modification Plate [\#] Name Gel ldx/Pos [4700 $\quad$ iTRAQ iTRAQ iTRAQ Rank Result Type

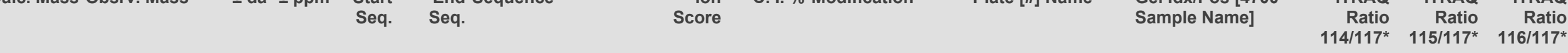

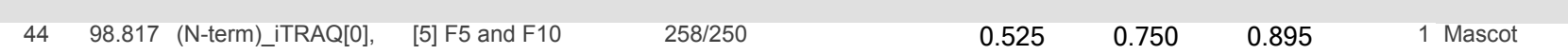


$\begin{array}{llllll}1305.646 & 1305.6111 & -0.0349 & -27 & 56 & 65 \\ \text { DGSYGAWLHR }\end{array}$

$\begin{array}{lllllll}1655.9254 & 1655.9398 & 0.0144 & 9 & 66 & 77 & \text { DSSTWLTAFVLK }\end{array}$

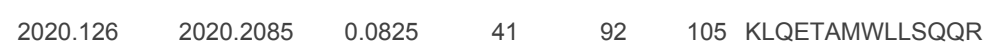

$\begin{array}{llllll}2380.3135 & 2380.197 & -0.1165 & -49 & 20 & 35 \\ \text { YLDKTEQWSLLPPETK }\end{array}$
4799.468 Lysine(K)_iTRAQ[7]

99.986 (N-term) iTRAQ[0], [4] F11 and F3 1279/1271

$\begin{aligned} & 38 \\ & 95.783 \\ & \begin{array}{l}\text { (N-term) iTRAQ[0], } \\ \text { Lysine(K) iTRAQ[1] }\end{array}\end{aligned} \quad[3] \mathrm{F} 14,15,16$ and F6 $\quad 1022 / 1014$

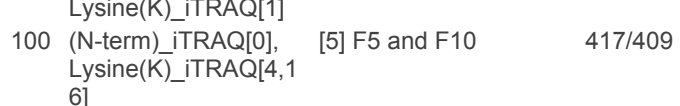

$\begin{array}{llll}0.701 & 0.715 & 1.110 & 1 \text { Masco }\end{array}$

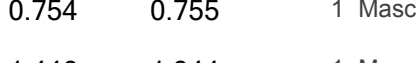

$\begin{array}{lll}1.446 & 1.344 \quad 1 \text { Masco }\end{array}$

$\begin{array}{llll}0.880 & 1.062 \quad 1.308 \quad 1 \text { Mascot }\end{array}$

37 Chain A, Crystal Structure Of The First Active gi|1942351 14234.1

$\begin{array}{lllll}286 & 0.738 & 0.640 & 0.908 & 0.329\end{array}$

0.307

0.067

Protein Group

Chain A, Complex Of The Second Kunitz Domain Of gi|2914482

Chain A The Refined 16 Angstroms Resoltion Crystal gil494360

Chain B, Refined 1.8 Angstroms Resolution Crystal gi|999627 $\quad 9481.9$

Structure Of Porcine Epsilon-Trypsin
RecName: Full=Trypsin; Flags: Precursor

\begin{tabular}{|c|c|c|c|c|c|}
\hline \multirow{2}{*}{\multicolumn{2}{|c|}{$\begin{array}{l}\text { Peptide Information } \\
\text { Calc. Mass Obsrv. Mass }\end{array}$}} & \multirow{2}{*}{\multicolumn{2}{|c|}{ \pm da \pm ppm }} & \multirow{2}{*}{$\begin{array}{l}\text { Start } \\
\text { Seq. }\end{array}$} & \multirow{2}{*}{$\begin{array}{l}\text { End Sequence } \\
\text { Seq. }\end{array}$} \\
\hline & & & & & \\
\hline 1189.6661 & 1189.6781 & 0.012 & 10 & 90 & 99 LSSPATLNSR \\
\hline 2499.3088 & 2499.1692 & -0.1396 & -56 & 50 & 69 LGEHNIDVLEGNEQFINA \\
\hline 2499.3088 & 2499.2151 & -0.0937 & -37 & 50 & :HNIDVLEGNEQFINA \\
\hline 2499.3088 & 2499.4016 & 0.0928 & 37 & 50 & 69 LGEHNIDVLEGNEQFINA \\
\hline 2571.385 & 2571.4092 & 0.0242 & 9 & $70 \mathrm{C}=\mathrm{s}$ & 89 IITHPNFI \\
\hline
\end{tabular}

$\begin{array}{lllll}\text { lon } & \text { C. I. \% Modification Plate [\#] Name Gel Idx/Pos [4700 iTRAQ iTRAQ iTRAQ Rank Result Type }\end{array}$

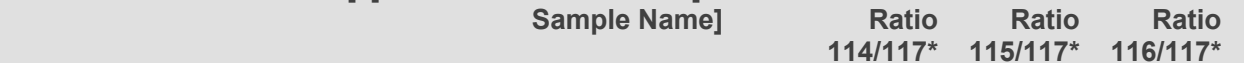

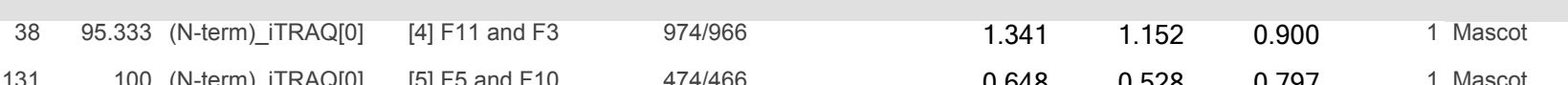

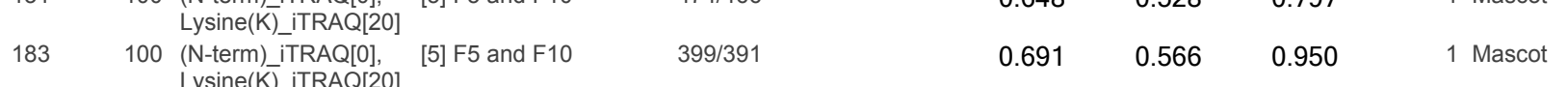

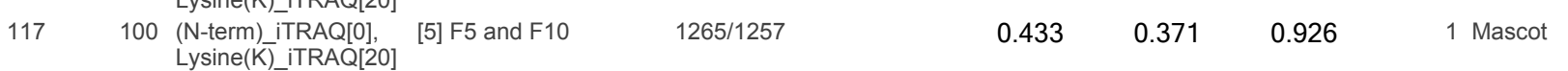

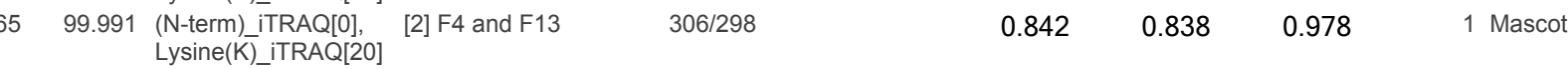

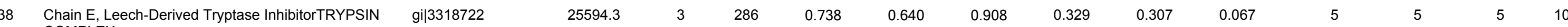
Peptide Information

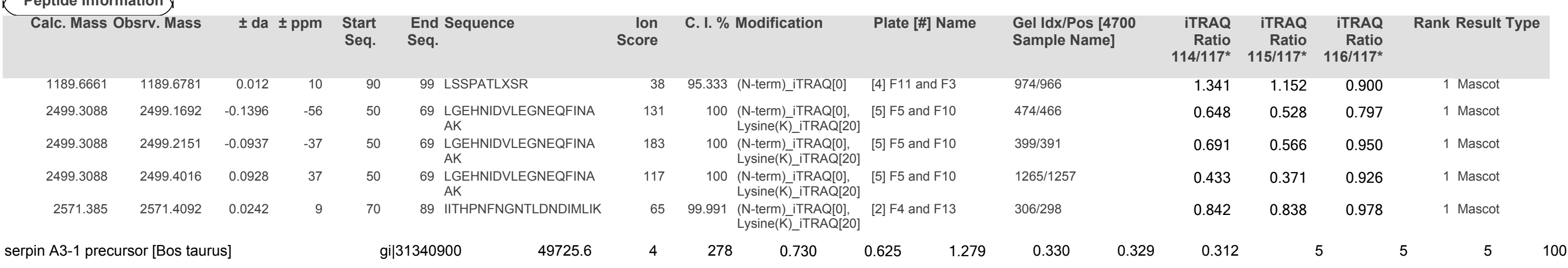

39 serpin A3-1 precursor [Bos taurus

Peptide Information

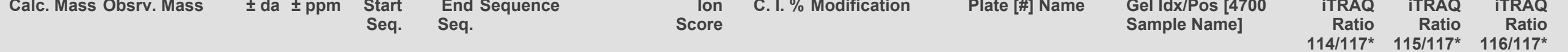

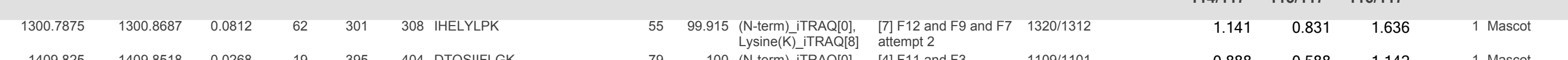

$\begin{array}{llllll}1409.825 & 1409.8518 & 0.0268 & 19 & 395 & 404 \\ \text { DTOSIFLCK }\end{array}$

$79100(\mathrm{~N}$ term)

\begin{tabular}{llllll}
1548.9094 & 1548.8564 & -0.053 & -34 & 87 & 98 \\
\hline
\end{tabular}

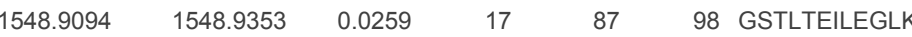

$\begin{array}{lllllll}16249645 & 1624.9529 & -0.0116 & -7 & 182 & 191 & \text { TOGKIEELFK }\end{array}$

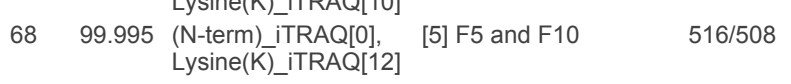

88100 (N-term) iTRAQ[0], [4] F11 and F3 1272/1264

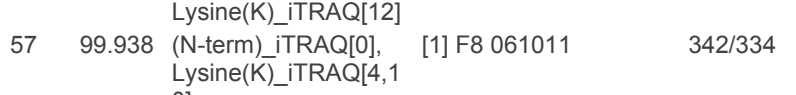

40 amyloid beta A4 protein isoform b precursor [Homo gil41406055 91356.8 01

$1236 \quad 0.919 \quad 0.950$

$\begin{array}{lll}0.409 & 0.412 & 0.987\end{array}$

$0.553-0.396 \quad 1.102-11000$

$\begin{array}{llll}0.904 & 1.201 & 1.682 & 1 \text { Mascot }\end{array}$

sapiens]

Protein Group

App protein [Rattus norvegicus]

gi|871360

84843.2

gi|38303889

89480.9 
amyloid beta A4 protein [Pan troglodytes]

hippocampal amyloid precursor protein [Mus musculus] gi|1805299 84681.2

$\begin{array}{lll}\text { putative amyloid precursor protein [Cavia sp.] } \quad \text { gil1418676 } & 85111.5\end{array}$

unnamed protein product [Rattus rattus] $\quad$ gi|55617 84749.4

\section{Peptide Information}

\begin{tabular}{|c|c|c|c|c|c|c|c|c|c|c|c|c|c|c|}
\hline Calc. Mass 0 & bsrv. Mass & \pm da & ppm & $\begin{array}{l}\text { Start } \\
\text { Seq. }\end{array}$ & $\begin{array}{l}\text { End Sequence } \\
\text { Seq. }\end{array}$ & $\begin{array}{l}\text { Ion } \\
\text { Score }\end{array}$ & c. $1 . \%$ & Modification & Plate [\#] Name & $\begin{array}{l}\text { Gel IdX/Pos [4700 } \\
\text { Sample Name] }\end{array}$ & $\begin{array}{r}\text { iTRAQ } \\
\text { Ratio } \\
114 / 117^{*}\end{array}$ & $\begin{array}{r}\text { iTRAQ } \\
\text { Ratio } \\
115 / 117^{*}\end{array}$ & $\begin{array}{r}\text { iTRAQ } \\
\text { Ratio } \\
116 / 117^{*}\end{array}$ & Rank Result Type \\
\hline 1387.7943 & 1387.745 & -0.0493 & -36 & 411 & 419 AVIQHFQEK & 63 & 99.986 & $\begin{array}{l}\text { (N-term) iTRAQ[0], } \\
\text { Lysine(K) TiTRAQ99] }\end{array}$ & [1] F8 061011 & $218 / 210$ & 0.871 & 0.639 & 0.974 & 1 Mascot \\
\hline 1387.7943 & 1387.8531 & 0.0588 & 42 & 411 & 419 AVIQHFQEK & 48 & 99.495 & $\begin{array}{l}\text { (N-term) iTRAQ[0], } \\
\text { (N) }\end{array}$ & $\begin{array}{l}{[7] F 12 \text { and } F 9 \text { and } F 7} \\
\text { P temnt }\end{array}$ & $1234 / 1226$ & 1.282 & 0.803 & 0.658 & 1 Mascot \\
\hline 1518.752 & 1518.7815 & 0.0295 & 19 & 420 & 431 VESLEQEAANER & 50 & 99.727 & (N-term)_iTRAQ[0] & [4] F11 and F3 & 1015/1007 & 1.659 & 1.194 & 1.085 & 1 Mascot \\
\hline 2039.0784 & 2039.1174 & 0.039 & 19 & 117 & 132 CLVGEFVSDALLVPDK & 76 & 99.999 & 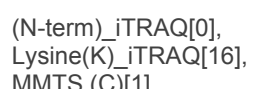 & [4] F11 and F3 & 1354/1346 & 1.587 & 1.220 & 1.298 & 1 Mascot \\
\hline 2211.084 & 2210.9844 & -0.0996 & -45 & 162 & 178 STNLHDYGMLLPCGIDK & 86 & 100 & $\begin{array}{l}\text { (N-term) iRARA[0], } \\
\text { Lysine(K) }(\text { iTRAQ[17], } \\
\text { MMTS (C)[13] }\end{array}$ & [5] F5 and F10 & $470 / 462$ & 0.983 & 0.877 & 0.855 & 1 Mascot \\
\hline a type IV c & a & $r[E$ & & & 81088.1 & 5 & 275 & 1.112 & 0.828 & 0.633 & 0.249 & & & 5 \\
\hline
\end{tabular}

$41 \quad 72 \mathrm{kDa}$ type IV collagenase precursor [Bos taurus] $\quad$ gi|27807447 81088.1 Peptide Information

\begin{tabular}{|c|c|c|c|c|c|c|c|c|c|c|c|c|c|}
\hline Calc. Mass C & srv. Mass & \pm da & ppm & $\begin{array}{l}\text { Start } \\
\text { Seq. }\end{array}$ & $\begin{array}{l}\text { End Sequence } \\
\text { Seq. }\end{array}$ & $\begin{array}{l}\text { lon } \\
\text { Score }\end{array}$ & C. I. \% Modification & Plate [\#] Name & $\begin{array}{l}\text { Gel Idx/Pos [4700 } \\
\text { Sample Name] }\end{array}$ & $\begin{array}{r}\text { iTRAQ } \\
\text { Ratio } \\
114 / 117^{*}\end{array}$ & $\begin{array}{r}\text { iTRAQ } \\
\text { Ratio } \\
115 / 117^{*}\end{array}$ & $\begin{array}{r}\text { iTRAQ } \\
\text { Ratio } \\
116 / 117^{*}\end{array}$ & Rank Result Type \\
\hline 1325.71 & 1325.6624 & -0.0476 & -36 & 569 & 577 VDAAFNWSK & 47 & $99.3699 \begin{array}{c}\text { N-term) } \\
\text { Lysine(iTRAR[0], }\end{array}$ & [5] F5 and F10 & $332 / 324$ & 2.493 & 0.947 & 0.960 & 1 Mascot \\
\hline 1562.8451 & 1562.8724 & 0.0273 & 17 & 148 & 159 AFQVWSDVTPLR & 46 & 99.269 (N-term)_iTRAQ[0] & [4] F11 and F3 & 1203/1195 & 1.013 & 1.076 & 1.260 & 1 Mascot \\
\hline 1695.8013 & 1695.8027 & 0.0014 & 1 & 177 & 188 WEHGDGYPFDGK & 65 & 99.991 (N-term)_iTRAQ[0], & [1] F8 061011 & 292/284 & 0.640 & 0.652 & 0.677 & 1 Mascot \\
\hline 1723.8636 & 1723.9016 & 0.038 & 22 & 521 & 532 IDAVYEDPQEEK & 70 & 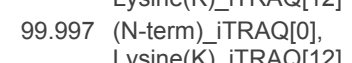 & [4] F11 and F3 & 1012/1004 & 1.172 & 0.950 & 1.175 & 1 Mascot \\
\hline 2568.3193 & 2568.2312 & -0.0881 & -34 & 46 & 63 TDKELAVQYLNTFYGCPK & 49 & 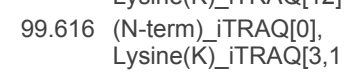 & [5] F5 and F10 & $492 / 484$ & 0.898 & 0.617 & 1.098 & 1 Mascot \\
\hline
\end{tabular}


42 phospholipid transfer protein [Bos taurus]

\section{sapiens]}

Peptide Information

\begin{tabular}{|c|c|c|c|c|c|c|c|c|c|c|c|c|c|}
\hline Calc. Mass & srv. Mass & \pm da & ppm & $\begin{array}{l}\text { Start } \\
\text { Seq. }\end{array}$ & $\begin{array}{l}\text { End Sequence } \\
\text { Seq. }\end{array}$ & $\begin{array}{l}\text { lon } \\
\text { Score }\end{array}$ & C. I. \% Modification & Plate [\#] Name & $\begin{array}{l}\text { Gel Idx/Pos [4700 } \\
\text { Sample Name] }\end{array}$ & $\begin{array}{r}\text { iTRAQ } \\
\text { Ratio } \\
114 / 117^{*}\end{array}$ & $\begin{array}{r}\text { iTRAQ } \\
\text { Ratio } \\
115 / 1117^{*}\end{array}$ & $\begin{array}{r}\text { iTRAQ } \\
\text { Ratio } \\
116 / 117^{*}\end{array}$ & Rank Result Type \\
\hline 1994.2385 & 1994.2294 & -0.0091 & -5 & 263 & 276 ITVVDALHEIPVKK & 66 & 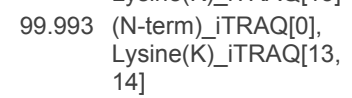 & [1] F8 061011 & $394 / 386$ & 1.122 & 0.809 & 1.463 & 1 Mascot \\
\hline 2574.1062 & 2573.9592 & -0.147 & -57 & 241 & $\begin{array}{l}262 \text { EDAGEYECHASNSQGQA } \\
\text { SASAK }\end{array}$ & 140 & 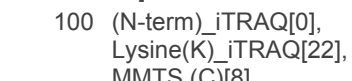 & [5] F5 and F10 & 202/194 & 0.292 & 0.376 & 0.705 & 1 Mascot \\
\hline
\end{tabular}

44 keratin, type II cytoskeletal 2 epidermal [Homo sapiens] gi|47132620 70810.8 Peptide Information

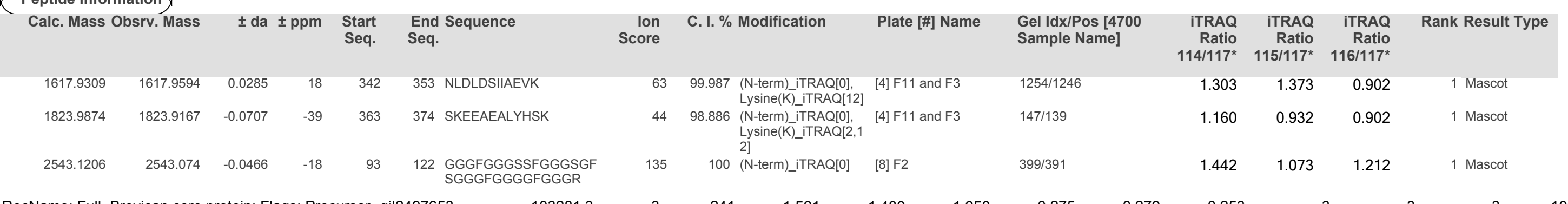

45 RecName: Full=Brevican core protein; Flags: Precursor gi|2497653 103281.3

\section{Peptide Information}

\begin{tabular}{|c|c|c|c|c|c|c|c|c|c|c|c|c|c|}
\hline Calc. Mass $\mathrm{C}$ & sirv. Mass & \pm da & :ppm & $\begin{array}{l}\text { Start } \\
\text { Seq. }\end{array}$ & $\begin{array}{l}\text { End Sequence } \\
\text { Seq. }\end{array}$ & $\begin{array}{l}\text { lon } \\
\text { Score }\end{array}$ & C. I. \% Modification & Plate [\#] Name & $\begin{array}{l}\text { Gel Idx/Pos [4700 } \\
\text { Sample Name] }\end{array}$ & $\begin{array}{r}\text { iTRAQ } \\
\text { Ratio } \\
114 / 117^{*}\end{array}$ & $\begin{array}{r}\text { iTRAQ } \\
\text { Ratio } \\
115 / 117^{*}\end{array}$ & $\begin{array}{r}\text { iTRAQ } \\
\text { Ratio } \\
116 / 117^{*}\end{array}$ & Rank Result Type \\
\hline 1027.6299 & 1027.626 & -0.0039 & -4 & 894 & 899 LVGHWK & 39 & $96.772 \begin{array}{l}\text { (N-term) iTRAQ[0], } \\
\text { Lysine(K) TiTRA[G] }\end{array}$ & [5] F5 and F10 & $1132 / 1124$ & 1.302 & 1.822 & 1.520 & 1 Mascot \\
\hline 1951.9998 & 1951.9644 & -0.0354 & -18 & 284 & 300 IATTGQLYAAWDGGLDR & 94 & 100 (N-term)_iTRAQ[0] & [8] F2 & $440 / 432$ & 1.915 & 1.512 & 1.334 & 1 Mascot \\
\hline
\end{tabular}

46 RecName: Full=Fibrinogen beta chain Contains:

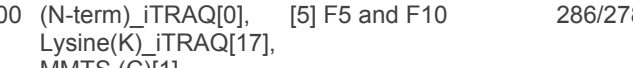

$\begin{array}{lll}\text { RecName: Full=Fibrinogen beta chain; Contains: } & \text { gil1346006 } & \\ \text { RecName: Full=Fibrinopeptide B; Contains: RecName: } & & \\ \text { F } & & \\ \begin{array}{lll}\text { Choin B, The Crystal Structure Of Modified Bovine } & \text { gil6980815 } & 52216.1 \\ \begin{array}{l}\text { Fibrinogen (At } 4 \text { Angstrom Resolution) } \\ \text { fibrinogen beta chain [Bos taurus] }\end{array} & \text { gil } 357 & 54018\end{array}\end{array}$

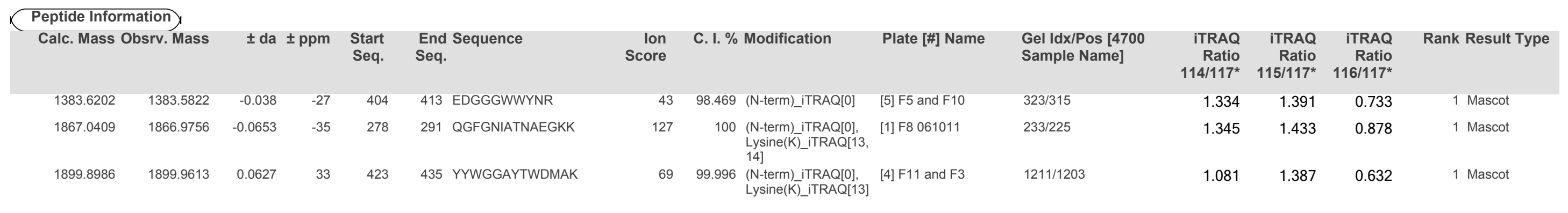


Short=ApoA-l; AttName: Full=Apolipoprotein A1; Fla

gi|3915607

33204

$3 \quad 232$

0.826

0.890

0.907

$0.241 \quad 0.303$

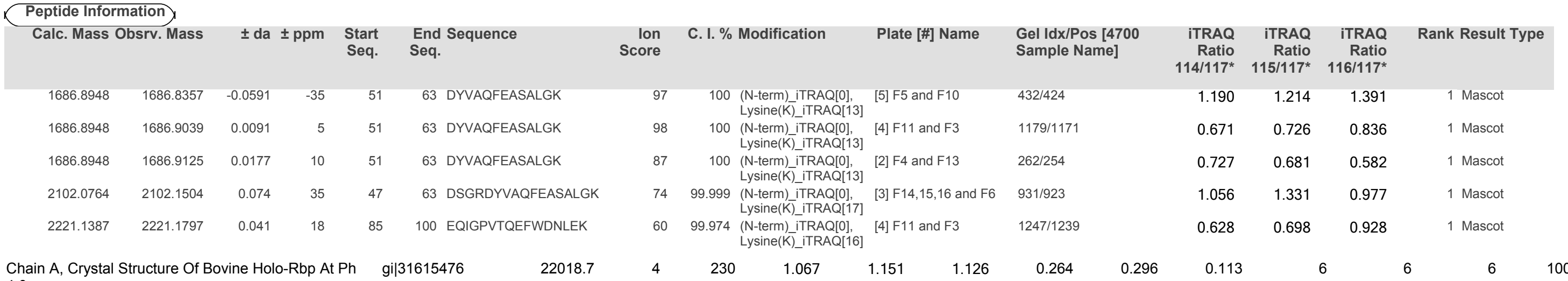

48 Chain A, Crystal Structure Of Bovine Holo-Rbp At Ph gi|31615476 22018.7

Protein Group

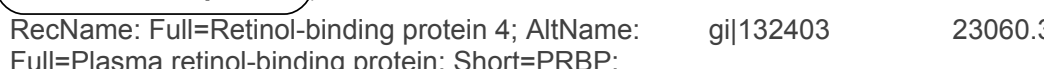

Full=Plasma retinol-binding

\begin{tabular}{|c|c|c|c|c|c|c|c|c|c|c|c|c|c|}
\hline Calc. Mass & osrv. Mass & \pm da & $\pm \mathrm{ppm}$ & $\begin{array}{l}\text { Start } \\
\text { Seq. }\end{array}$ & $\begin{array}{l}\text { End Sequence } \\
\text { Seq. }\end{array}$ & $\begin{array}{l}\text { Ion } \\
\text { Score }\end{array}$ & C. I. \% Modification & Plate [\#] Name & $\begin{array}{l}\text { Gel Id d/Pos [4700 } \\
\text { Sample Name] }\end{array}$ & $\begin{array}{r}\text { iTRAQ } \\
\text { Ratio } \\
114 / 117^{*}\end{array}$ & $\begin{array}{r}\text { iTRAQ } \\
\text { Ratio } \\
115 / 117^{*}\end{array}$ & $\begin{array}{r}\text { iTRAQ } \\
\text { Ratio } \\
116 / 117^{*}\end{array}$ & Rank Result Type \\
\hline 1311.7644 & 1311.7521 & -0.0123 & -9 & 11 & 17 VKENFDK & 40 & 97.162 (N-term) iTRAQ[0], & [5] F5 and F10 & 1041/1033 & 1.030 & 1.314 & 1.266 & 1 Mascot \\
\hline 1433.7498 & 1433.7698 & 0.02 & 14 & 20 & 29 FAGTWYAMAK & 61 & 99.977 (N-term) iTRAQ[0], & [2] F4 and F13 & 222/214 & 1.375 & 1.470 & 1.046 & 1 Mascot \\
\hline 1478.7737 & 1478.8044 & 0.0307 & 21 & 140 & 150 DPSGFSPEVQK & 42 & 98.134 (N-term) _TRAQ[O], & [4] F11 and F3 & 995/987 & 1.012 & 0.857 & 1.065 & 1 Mascot \\
\hline 1478.7737 & 1478.8202 & 0.0465 & 31 & 140 & 150 DPSGFSPEVQK & 57 & 99.94 (N-term) iTRAQ[0], & [2] F4 and F13 & 913/905 & 0.724 & 0.885 & 1.092 & 1 Mascot \\
\hline 1486.8304 & 1486.8406 & 0.0102 & 7 & 90 & 99 YWGVASFLQK & 57 & 99.938 (N-term) iTRAQ[O], & [4] F11 and F3 & $1228 / 1220$ & 1.399 & 1.051 & 1.019 & 1 Mascot \\
\hline 1486.8304 & 1486.8527 & 0.0223 & 15 & 90 & 99 YWGVASFLQK & 72 & 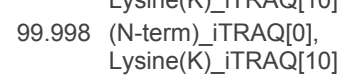 & [2] F4 and F13 & $308 / 300$ & 1.013 & 1.506 & 1.302 & 1 Mascot \\
\hline
\end{tabular}

49 complement component 4, gene 2 [Rattus norvegicus] gi|50657362 205542.6

\section{Peptide Information}

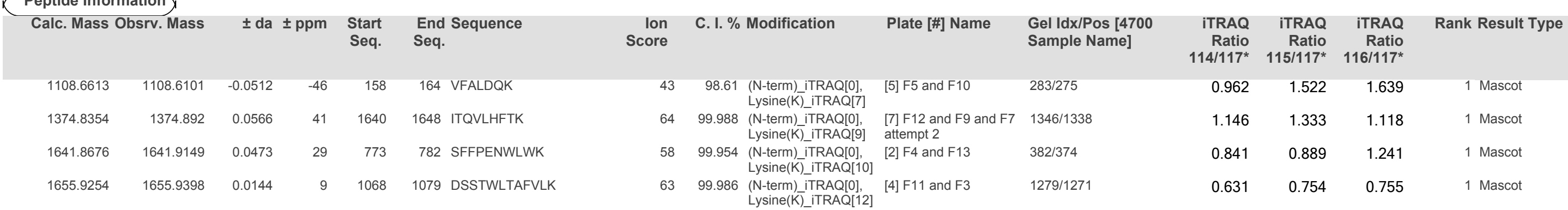

\section{0 hypothetical protein [Homo sapiens]}$$
\text { gi|30722344 263094 }
$$

Protein Group

fibronectin isoform 1 preproprotein [Homo sapiens]

gil53791223

263248.7

fibronectin isoform 3 preproprotein [Homo sapiens]

$132557 \quad 286441.2$

fibronectin isoform 4 preproprotein [Homo sapiens]

273344.7

fibronectin isoform 5 preproprotein [Homo sapiens]

fibronectin isoform 6 preproprotein [Homo sapiens]

fibronectin precursor [Homo sapiens]

gi|47132553 $\quad 266655.4$

gi|47132549

gi|31397

253334.6

hypothetical protein [Homo sapiens]

hypothetical protein [Homo sapiens]

gi|31873670

283329.9

hypothetical protein [Homo sapiens]

gi|34364820

283118.8 
Peptide Information

\begin{tabular}{|c|c|c|c|c|c|c|c|c|c|c|c|c|c|}
\hline \multicolumn{2}{|c|}{ Calc. Mass Obsrv. Mass } & \multicolumn{2}{|c|}{ \pm da $\pm p p m$} & \multirow{2}{*}{$\begin{array}{r}\begin{array}{l}\text { Start } \\
\text { Seq. }\end{array} \\
147\end{array}$} & \multirow{2}{*}{$\begin{array}{l}\text { End Sequence } \\
\text { Seq. } \\
156 \text { HYQINQQWER }\end{array}$} & \multirow{2}{*}{$\begin{array}{r}\begin{array}{r}\text { Ion } \\
\text { Score }\end{array} \\
40\end{array}$} & \multirow{2}{*}{$\begin{array}{l}\text { C. I. \% Modification } \\
96.86 \text { (N-term)_iTRAQ[0] }\end{array}$} & \multirow{2}{*}{$\begin{array}{l}\text { Plate [\#] Name } \\
\text { [1] F8 } 061011\end{array}$} & \multirow{2}{*}{$\begin{array}{l}\text { Gel Idx/Pos [4700 } \\
\text { Sample Name] } \\
245 / 237\end{array}$} & \multirow{2}{*}{$\begin{array}{r}\text { iTRAQ } \\
\text { Ratio } \\
114 / 117^{*} \\
1.194\end{array}$} & \multirow{2}{*}{$\begin{array}{r}\text { iTRAQ } \\
\text { Ratio } \\
115 / 117^{*} \\
1.086\end{array}$} & \multirow{2}{*}{$\begin{array}{r}\text { iTRAQ } \\
\text { Ratio } \\
116 / 117^{*} \\
0.692\end{array}$} & \multirow{2}{*}{$\begin{array}{c}\text { Rank Result Type } \\
1 \text { Mascot }\end{array}$} \\
\hline 1545.7683 & 1545.7308 & -0.0375 & -24 & & & & & & & & & & \\
\hline 1773.9731 & 1773.9127 & -0.0604 & -34 & 1028 & 1042 VDVIPVNLPGEHGQR & 51 & 99.767 (N-term)_iTRAQ[0] & [5] F5 and F10 & $346 / 338$ & 0.674 & 0.657 & 0.731 & 1 Mascot \\
\hline 2405.3208 & 2405.2563 & -0.0645 & -27 & 1766 & $\begin{array}{l}1786 \text { EINLAPDSSSVVVSGLMV } \\
\text { ATK }\end{array}$ & 138 & 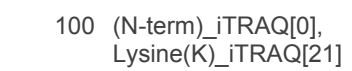 & {$[8] \mathrm{F} 2$} & $472 / 464$ & 1.162 & 0.630 & 1.119 & 1 Mascot \\
\hline atin $10[$ Hon & piens] & & & & 1961605 & 3 & 2.039 & 1.674 & 0.703 & 0.277 & & 3 & 3 \\
\hline
\end{tabular}

51 Keratin 10 [Homo sapiens]

Peptide Information

$\begin{array}{lllllllll}\text { Calc. Mass Obsrv. Mass } & \pm d a \pm p p m & \text { Start } & \text { End Sequence } & \text { Ion } & \text { C. I. \% Modification } & \text { Plate [\#] Name } & \text { Gel ldx/Pos }[4700 & \text { iTRAQ iTRAQ iTRAQ Rank Result Type }\end{array}$ $\begin{array}{lll}\text { Seq. } & \text { Seq. } & \end{array}$

\begin{tabular}{llllll}
1397.6947 & 1397.656 & -0.0387 & -28 & 335 & 343 \\
\hline
\end{tabular}

$\begin{array}{llllll}1427.827 & 1427.9156 & 0.0886 & 62 & 178 & 184 \\ \text { IKEWYEK }\end{array}$

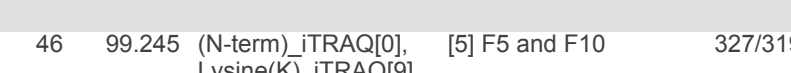

$\begin{array}{lllllll}2476.051 & 2476.0151 & -0.0359 & -14 & 60 & 86 & \text { GSSGGGCFGGSSGGYC }\end{array}$

$44 \quad 98.878$ (N-term) iTRAQ[0], $\quad$ [7] F12 and F9 and F7 $907 / 899$

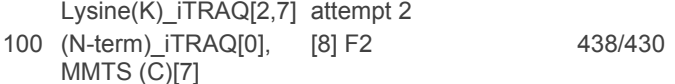

52 RecName: Full=Hemoglobin subunit beta; AltName: gil122686 17982

Full=Beta-globin; Altname:

17982.6

Peptide Information

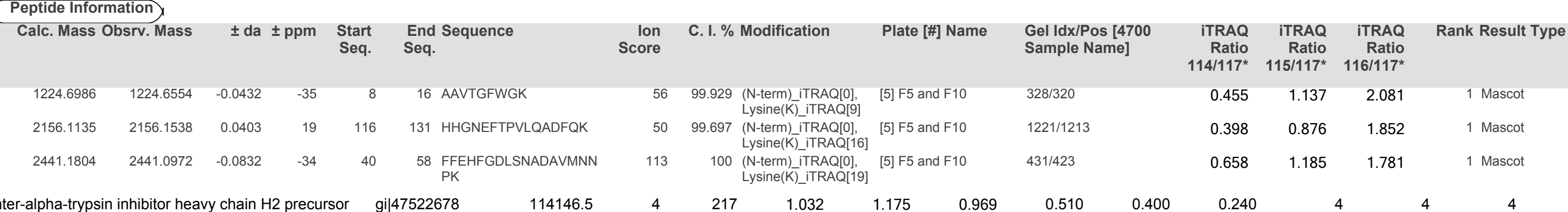

53 inter-alpha-trypsin inhibitor heavy chain $\mathrm{H} 2$ precursor $\quad$ gi|47522678 114146.5

$\begin{array}{rrr}\text { Ratio } & \text { Ratio } & \text { Ratio } \\ 114 / 117^{*} & 115 / 117^{*} & 116 / 117^{*}\end{array}$

$\begin{array}{llll}1.571 & 1.362 & 0.757 & 1 \text { Mascot }\end{array}$

$\begin{array}{llll}3.086 & 2.265 & 1.319 & 1 \text { Mascot }\end{array}$

$\begin{array}{llll}1.748 & 1.520 & 1.139 \quad 1 \text { Mascot }\end{array}$

\section{Peptide Information}

\begin{tabular}{|c|c|c|c|c|c|c|c|c|c|c|c|c|c|}
\hline \multicolumn{2}{|c|}{ Calc. Mass Obsrv. Mass } & \multicolumn{2}{|c|}{ \pm da $\pm \mathrm{ppm}$} & $\begin{array}{l}\text { Start } \\
\text { Seq. }\end{array}$ & $\begin{array}{l}\text { End Sequence } \\
\text { Seq. }\end{array}$ & $\begin{array}{l}\text { lon } \\
\text { Score }\end{array}$ & C. I. \% Modification & Plate [\#] Name & $\begin{array}{l}\text { Gel Idx/Pos [4700 } \\
\text { Sample Name] }\end{array}$ & $\begin{array}{r}\text { iTRAQ } \\
\text { Ratio } \\
114 / 117^{*}\end{array}$ & $\begin{array}{r}\text { iTRAQ } \\
\text { Ratio } \\
115 / 117^{*}\end{array}$ & $\begin{array}{r}\text { iTRAQ } \\
\text { Ratio } \\
116 / 117^{*}\end{array}$ & Rank Result Type \\
\hline 1707.9315 & 1707.9822 & 0.0507 & 30 & 166 & 176 VQFELHYQEVK & 45 & 99.121 (N-term) -TRAQ[0], & {$[3] \mathrm{F} 14,15,16$ and $F 6$} & $857 / 849$ & 0.851 & 0.781 & 0.690 & 1 Mascot \\
\hline 1741.0118 & 1741.0032 & -0.0086 & -5 & 236 & 248 AHVAFKPTVAQQR & 42 & 98.294 (N-term) -iTRAQ[0], & [5] F5 and F10 & 1093/1085 & 0.656 & 1.208 & 1.195 & 1 Mascot \\
\hline
\end{tabular}

54 neurosecretory protein VGF precursor [Homo sapiens] gil17136078 69569.2

98.294 (N-term) ITRAQ[0], [5] F5 and F10 1093/1085

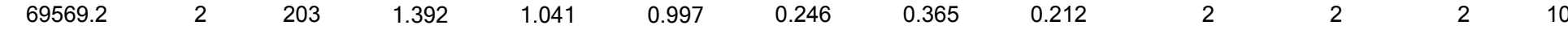

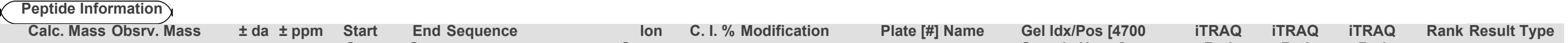

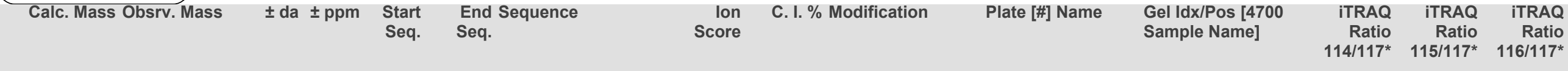

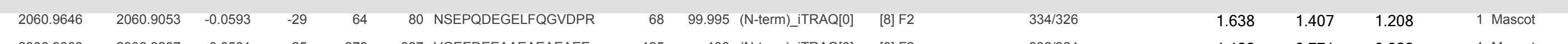
$\begin{array}{lllllllllllllll}2306.9868 & 2306.9287 & -0.0581 & -25 & 378 & 397 & \text { VGEEDEEAAEAEAEAEE } & 135 & 100 \text { (N-term)iTRAQ[0] } & {[8] \text { Fe } 2} & 332 / 324 & 1.182 & 0.771 & 0.822 & 1 \text { Mascot }\end{array}$

\begin{tabular}{llllllllllllll}
55 & fibronectin [Sus scrofa] & gil56608605 & 69908.3 & 2 & 199 & 1.320 & 0.881 & 1.098 & 0.179 & 0.351 & 0.021 & 2 & 2 \\
\hline
\end{tabular} Pribronectin [Sus scrofa]

Peptide Information

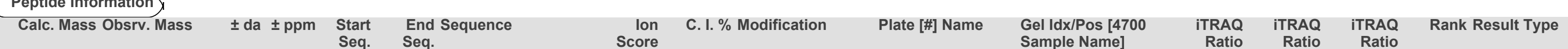




\begin{tabular}{|c|c|c|c|c|c|c|c|c|c|c|c|c|c|}
\hline & & & & & & & & & & 14/117 & 15/117 & $116 / 117^{*}$ & \\
\hline 1575.8826 & 1575.8358 & -0.0468 & -30 & 405 & 419 VPGTSASATLTGLTR & 62 & 99.981 (N-term)_iTRAQ[0] & [8] F2 & $336 / 328$ & 1.499 & 1.231 & 1.078 & 1 Mascot \\
\hline 2405.3208 & 2405.2563 & -0.0645 & -27 & 47 & $\begin{array}{l}67 \text { EINLAPDSSSVVVSGLMV } \\
\text { ATK }\end{array}$ & 138 & $\begin{array}{l}100 \begin{array}{l}\text { (N-term)_iTRAQ[0], } \\
\text { Lysine(K)_TRAQ[21] }\end{array}\end{array}$ & {$[8]$ F2 } & 472/464 & 1.162 & 0.630 & 1.119 & 1 Mascot \\
\hline
\end{tabular}

56 prothrombin [Bos taurus] gi|27806947 $\quad 76293.7$

$196-1.018-0.898$

Protein Group
RecName: Full=Prothrombin; AltName:
Full=Coagulation factor II; Contains: RecName:

gi|135806

76221.6

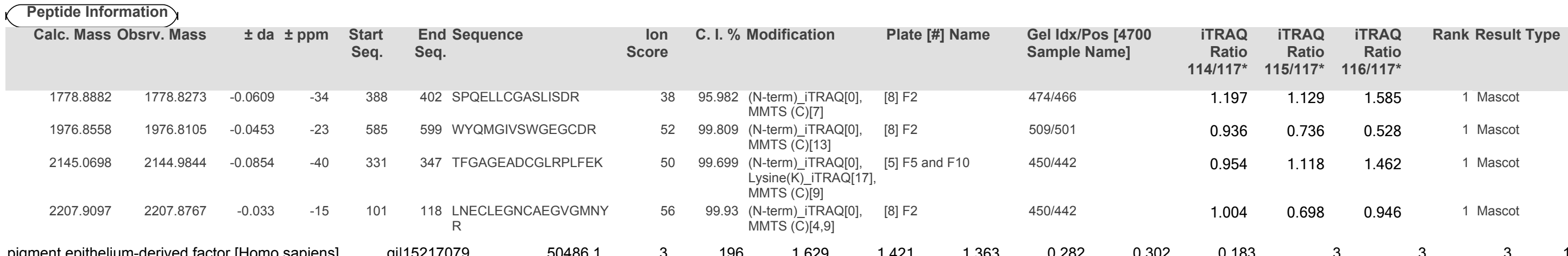

57 pigment epithelium-derived factor [Homo sapiens] $\quad$ gi|15217079 50486.1

$\begin{array}{llll}3 & 196 & 1.629 & 1.421\end{array}$

$1.363 \quad 0.282$

Protein Group

Chain A, 285 A Crystal Structure Of Pedf

gi|15988024

48330.1

Serpin peptidase inhibitor, clade F (alpha-2 antiplasmin, gil15559258 50545.2

pigment epithelium derived factor), membe

pigment epithelial-differentiating factor [Homo sapiens] gi|189778

50473.1

pigment epithelium-derived factor [Homo sapiens] $\quad$ gil1144299 $\quad 44074.8$

pigment epithelium-derived factor precursor [Homo $\quad$ gil39725934 $\quad 50456.1$

Peptide Information

\begin{tabular}{|c|c|c|c|c|c|c|c|c|c|c|c|c|c|}
\hline \multicolumn{2}{|c|}{ Calc. Mass Obsrv. Mass } & \multicolumn{2}{|c|}{$\pm \mathrm{da} \pm \mathrm{ppm}$} & \multirow{2}{*}{$\begin{array}{r}\begin{array}{l}\text { Start } \\
\text { Seq. }\end{array} \\
346\end{array}$} & \multirow{2}{*}{$\begin{array}{l}\text { End Sequence } \\
\text { Seq. } \\
352 \text { ITGKPIK }\end{array}$} & \multirow{2}{*}{$\begin{array}{r}\begin{array}{r}\text { Ion } \\
\text { Score }\end{array} \\
\quad 38\end{array}$} & \multirow{2}{*}{ 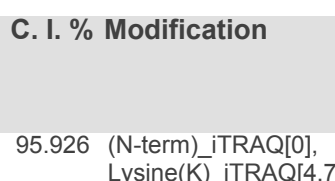 } & Plate [\#] Name & $\begin{array}{l}\text { Gel Idx/Pos [4700 } \\
\text { Sample Name] }\end{array}$ & \multirow{2}{*}{$\begin{array}{r}\text { iTRAQ } \\
\text { Ratio } \\
114 / 117^{*} \\
2.031\end{array}$} & \multirow{2}{*}{ 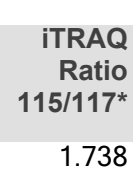 } & \multirow{2}{*}{$\begin{array}{r}\text { iTRAQ } \\
\text { Ratio } \\
116 / 117^{*} \\
1.551\end{array}$} & \multirow{2}{*}{$\begin{array}{c}\text { Rank Result Type } \\
1 \text { Mascot }\end{array}$} \\
\hline 1188.8052 & 1188.8368 & 0.0316 & 27 & & & & & $\begin{array}{l}\text { [7] F12 and F9 and F7 } \\
\text { ] attempt } 2 \text {. }\end{array}$ & $817 / 809$ & & & & \\
\hline 1703.8877 & 1703.8383 & -0.0494 & -29 & 54 & 67 LAAAVSNFGYDLYR & 80 & 100 (N-term)_iTRAQ[0] & [8] F2 & 469/4461 & 1.521 & 1.507 & 1.149 & 1 Mascot \\
\hline 2118.1262 & 2118.1643 & 0.0381 & 18 & 175 & 189 LDLQEINNWVQAQMK & 77 & 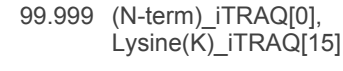 & [4] F11 and F3 & $1324 / 1316$ & 1.400 & 1.095 & 1.422 & 1 Mascot \\
\hline or XIla inhibi & recursc & staurus & & & 55333.2 & 3 & 1.161 & 1.276 & 0.252 & 0.278 & 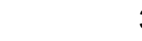 & 3 & 3 \\
\hline
\end{tabular}

58 factor XIla inhibitor precursor [Bos taurus] gi|27807349 55333.2

Peptide Information

$\begin{array}{llllllll}\text { Calc. Mass Obsrv. Mass } \pm \text { da } \pm \text { ppm } & \text { Start } & \text { End Sequence } & \text { lon } & \text { C. I. \% Modification Plate [\#] Name } & \text { Gelldx/Pos [4700 } & \text { iTRAQ iTRAQ iTRAQ Rank Result Type }\end{array}$

$\begin{array}{llll}\text { Calc. Mass Obsrv. Mass } \pm \mathrm{da} \pm \mathrm{ppm} & \begin{array}{l}\text { Start } \\ \text { Seq. }\end{array} & \begin{array}{l}\text { End Sequence } \\ \text { Seq. }\end{array}\end{array}$ Score

$\begin{array}{lrrr}\text { Gel ldx/Pos [4700 } & \text { Ratio } & \text { Ratio } & \text { Ratio } \\ \text { Sample Name] } & 114 / 117^{*} & 115 / 117^{*} & 116 / 117^{*}\end{array}$

$\begin{array}{rrrrrl}1380.733 & 1380.672 & -0.061 & -44 & 365 & 374 \text { FHPTHLTMPR } \\ 1513.9927 & 1514.0712 & 0.0785 & 52 & 261 & 272 \text { LILLNAVALSAK }\end{array}$

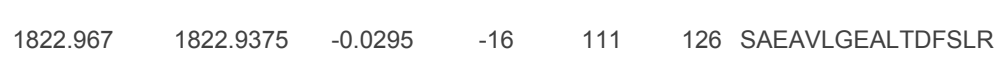

$40 \quad 97.412$ (N-term)_iTRAQ[0] [4] F11 and F3 $\quad 203 / 195$

85100 (N-term)_iTRAQ[0], [4] F11 and F3 $1257 / 1249$

$558 / 550$

$\begin{array}{rrr}114 / 117^{*} & 115 / 117^{*} & 116 / 117^{*} \\ 0.917 & 0.993 & 0.986\end{array}$

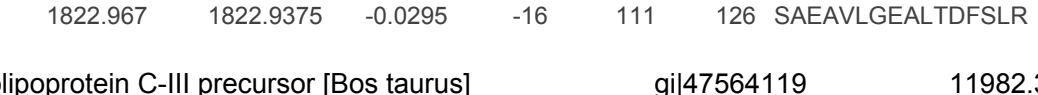

6899.996 (N-term) iTRAQ[0] [8] F2

$\begin{array}{llll}0.917 & 0.993 & 0.986 & 1 \text { Mascot } \\ 1.484 & 0.994 & 1.324 & 1 \text { Mascot }\end{array}$

Peptide Information

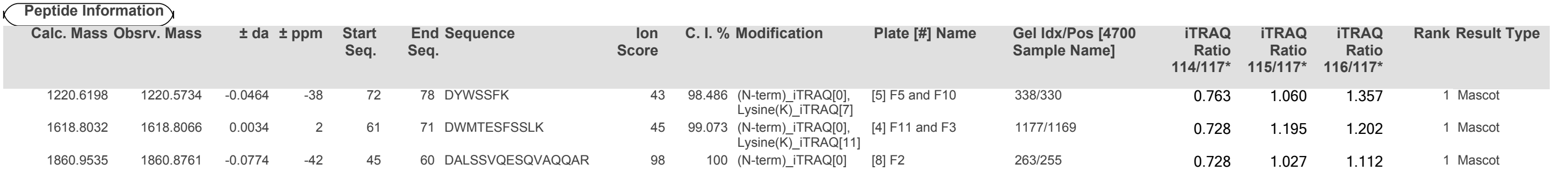


60 primary amine oxidase, lung isozyme [Bos taurus] Protein Group

i|30794300

\begin{tabular}{l}
$\begin{array}{l}\text { primary amine oxidase, liver isozyme precursor [Bos } \\
\text { taurus] }\end{array}$ gil195539525 87756.5 \\
\hline
\end{tabular}

\section{Peptide Information}

\begin{tabular}{|c|c|c|c|c|c|c|c|c|c|c|c|c|c|}
\hline Calc. Mass & bsrv. Mass & \pm da & \pm ppm & $\begin{array}{l}\text { Start } \\
\text { Seq. }\end{array}$ & $\begin{array}{l}\text { End Sequence } \\
\text { Seq. }\end{array}$ & $\begin{array}{l}\text { Ion } \\
\text { Score }\end{array}$ & C. I. \% Modification & Plate [\#] Name & $\begin{array}{l}\text { Gel Idx/Pos [4700 } \\
\text { Sample Name] }\end{array}$ & $\begin{array}{r}\text { iTRAQ } \\
\text { Ratio } \\
114 / 117^{*}\end{array}$ & $\begin{array}{r}\text { iTRAQ } \\
\text { Ratio } \\
115 / 117^{*}\end{array}$ & $\begin{array}{r}\text { iTRAQ } \\
\text { Ratio } \\
116 / 117^{*}\end{array}$ & Rank Result Type \\
\hline 1700.8438 & 1700.8082 & -0.0356 & -21 & 175 & 186 EYLDIDQMIFNR & 67 & 99.994 (N-term)_iTRAQ[0] & [8] F2 & $487 / 479$ & 0.803 & 0.904 & 1.284 & 1 Mascot \\
\hline 2045.0424 & 2044.9833 & -0.0591 & -29 & 568 & 585 QLETEEQAAFPLGGASP & 60 & 99.974 (N-term)_iTRAQ[0] & [8] F2 & $359 / 351$ & 0.643 & 0.811 & 0.972 & 1 Mascot \\
\hline 2317.2397 & 2317.1719 & -0.0678 & -29 & 567 & $\begin{array}{l}585 \text { KQLETEEQAAFPLGGAS } \\
\text { PR }\end{array}$ & 56 & 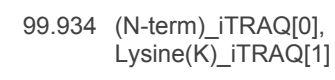 & [5] F5 and F10 & $326 / 318$ & 0.828 & 1.028 & 0.944 & 1 Mascot \\
\hline sex hormone-bin & ing globulin | & Bos taurus & & & 38325826 & 2 & 1.481 & 1.320 & 0.339 & 0.065 & & 2 & 2 \\
\hline Peptide Infor & ation & & & & & & & & & & & & \\
\hline Calc. Mass & bsrv. Mass & \pm da & $\pm \mathrm{ppm}$ & $\begin{array}{l}\text { Start } \\
\text { Seq. }\end{array}$ & $\begin{array}{l}\text { End Sequence } \\
\text { Seq. }\end{array}$ & $\begin{array}{l}\text { Ion } \\
\text { Score }\end{array}$ & C. I. \% Modification & Plate [\#] Name & $\begin{array}{l}\text { Gel Id I/Pos }[4700 \\
\text { Sample Name] }\end{array}$ & $\begin{array}{r}\text { iTRAQ } \\
\text { Ratio } \\
114 / 117^{*}\end{array}$ & $\begin{array}{r}\text { iTRAQ } \\
\text { Ratio } \\
115 / 117^{*}\end{array}$ & $\begin{array}{r}\text { iTRAQ } \\
\text { Ratio } \\
116 / 117^{*}\end{array}$ & Rank Result Type \\
\hline 1586.939 & 1586.912 & -0.027 & -17 & 164 & 177 IALGGLLFPASDLR & 51 & 99.758 (N-term)_iTRAQ[0] & [8] F2 & $534 / 526$ & 1.820 & 1.937 & 1.235 & 1 Mascot \\
\hline 2376.1553 & 2376.0896 & -0.0657 & -28 & 190 & 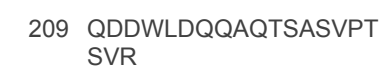 & 122 & 100 (N-term)_iTRAQ[0] & [8] F2 & $343 / 335$ & 1.206 & 0.899 & 1.109 & 1 Mascot \\
\hline
\end{tabular}

62 apolipoprotein A-IV precursor [Sus scrofa] gi|47523830 48023.

$\begin{array}{lll}171 & 1.087 & 0.983\end{array}$

\section{Peptide Information}

\begin{tabular}{|c|c|c|c|c|c|c|c|c|c|c|c|c|c|c|}
\hline Calc. Mass & srv. Mass & \pm da & ppm & $\begin{array}{l}\text { Start } \\
\text { Seq. }\end{array}$ & $\begin{array}{l}\text { End Sequence } \\
\text { Seq. }\end{array}$ & $\begin{array}{l}\text { lon } \\
\text { Score }\end{array}$ & C. I. $\%$ & Modification & Plate [\#] Name & $\begin{array}{l}\text { Gel Id d/Pos [4700 } \\
\text { Sample Name] }\end{array}$ & $\begin{array}{r}\text { iTRAQ } \\
\text { Ratio } \\
114 / 117^{*}\end{array}$ & $\begin{array}{r}\text { iTRAQ } \\
\text { Ratio } \\
115 / 117^{*}\end{array}$ & $\begin{array}{r}\text { iTRAQ } \\
\text { Ratio } \\
116 / 117^{*}\end{array}$ & Rank Result Type \\
\hline 1510.8839 & 1510.9172 & 0.0333 & 22 & 113 & 123 LLPHATEVSQK & 53 & 99.862 & $\begin{array}{l}\text { (N-term) iTRAQ[0], } \\
\text { Lysine(KK) iTRAQ[1]] }\end{array}$ & [3] F14,15,16 and F6 & $775 / 767$ & 1.101 & 0.804 & 0.990 & 1 Mascot \\
\hline 1510.8839 & 1510.9908 & 0.1069 & 71 & 113 & 123 LLPHATEVSQK & 72 & 99.998 & $\begin{array}{l}\text { (N-term) iTRAQ[0], } \\
\text { Lyysinen(K) }\end{array}$ & $\begin{array}{l}{[7] F 12 \text { and } F 9 \text { and } F 7} \\
\text { attempt } 2\end{array}$ & 1260/1252 & 0.794 & 0.892 & 0.841 & 1 Mascot \\
\hline 1816.9989 & 1817.0597 & 0.0608 & 33 & 234 & 246 LNHQLEGLAFQMK & 40 & 97.201 & (N-term) ITRAQ[0], & [3] F14,15,16 and F6 & 928/920 & 0.884 & 0.840 & 0.753 & 1 Mascot \\
\hline 1953.054 & 1953.0747 & 0.0207 & 11 & 52 & 65 SELTQQLNTLFQDK & 59 & 99.967 & 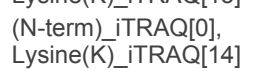 & [4] F11 and F3 & $1221 / 1213$ & 1.803 & 1.547 & 1.015 & 1 Mascot \\
\hline
\end{tabular}

63 complement C3 alpha chain [Oryctolagus cuniculus] gi|126723309 90597.5

\section{Peptide Information}

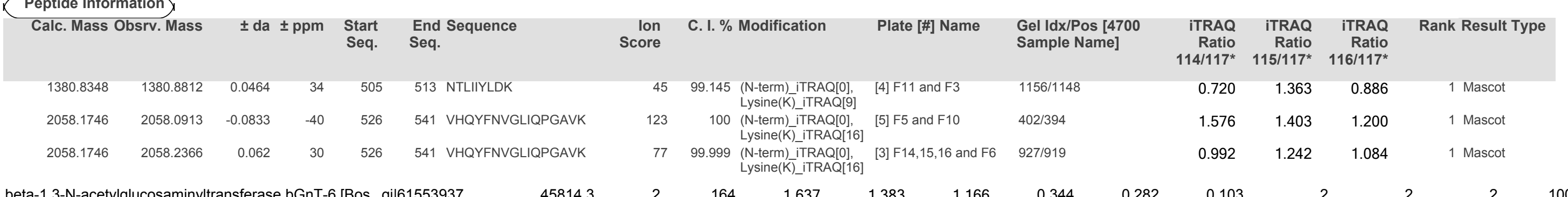

64 beta-1,3-N-acetylglucosaminyltransferase bGnT-6 [Bos gil61553937 45814.3

\section{beta-1,3-N
taurus] \\ Protein Group
$\begin{gathered}\text { N-acetyllactosaminide } \\ \text { beta-1,3-N-acetylglucosaminyltransferase [Bos taurus] }\end{gathered}$ gil77736590 49858.4}

$2 \quad 164 \quad 1.637$

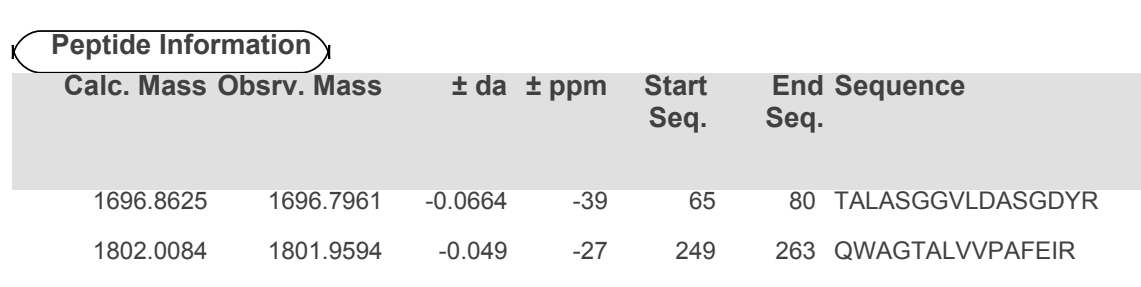$$
\begin{array}{llllll}
1802.0084 & 1801.9594 & -0.049 & -27 & 249 & 263 \\
\text { QWAGTALVVPAFEIR }
\end{array}
$$

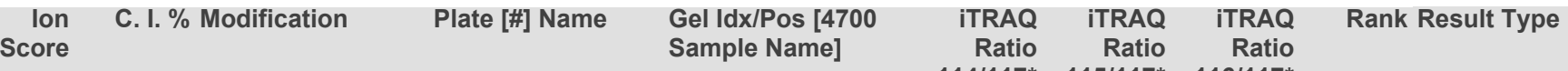

78092.2

RecName: Full=Serum al

Calc. Mass Obsrv. Mass $\quad \pm$ da \pm ppm Start End Sequence
4197.709 (N-term)_iTRAQ[0] $[8]$ F2

$\begin{array}{lrrr}\text { Gel Idx/Pos [4700 } & \begin{array}{r}\text { Ratio } \\ \text { Sample Name] }\end{array} & \begin{array}{r}\text { Ratio } \\ 114 / 117^{*}\end{array} & \begin{array}{r}\text { Ratio } \\ 115 / 117^{*} \\ 116 / 117^{*}\end{array}\end{array}$

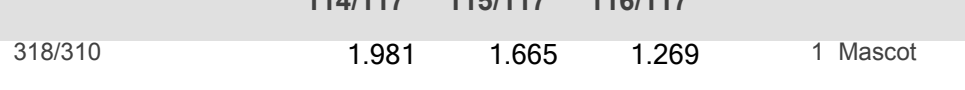

$\begin{array}{llll}1.353 & 1.149 & 1.072 & 1 \text { Mascot }\end{array}$ $\begin{array}{lllll}4 & 162 & 1.144 & 1.163 & 0.954\end{array}$ 


$\begin{array}{rrrrrc}1057.5802 & 1057.526 & -0.0542 & -51 & 154 & 160 \text { YLYEVAR } \\ 1077.6765 & 1077.7052 & 0.0287 & 27 & 250 & 256 \text { LVTDLTK } \\ 1077.6765 & 1077.7075 & 0.031 & 29 & 250 & 256 \text { LVTDLTK } \\ 1175.6508 & 1175.6292 & -0.0216 & -18 & 476 & 482 \text { LCVLHEK } \\ 1175.6508 & 1175.6975 & 0.0467 & 40 & 476 & 482 \text { LCVLHEK } \\ 1260.5291 & 1260.5676 & 0.0385 & 31 & 492 & 500 \text { CCTESLVNR }\end{array}$

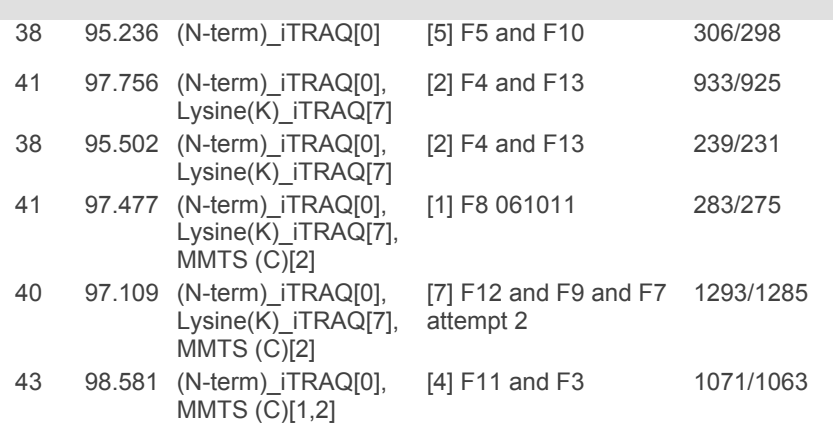

66 DKFZP459P137 protein [Pongo abelii]

\section{gi|207079999}

58525.5

Protein Group gi|1144316

58255.6

nucleobindin [Homo sapiens]

gil189308 $\quad 58165.5$

nucleobindin-1 precursor [Homo sapiens]

gi|20070228

58169.4

\section{Peptide Information}

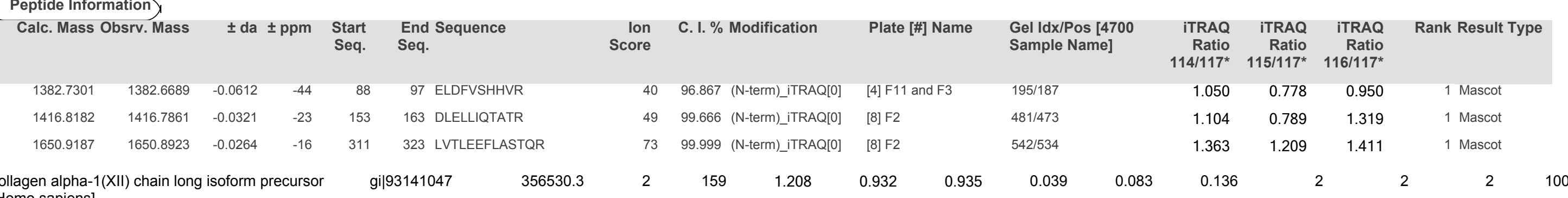

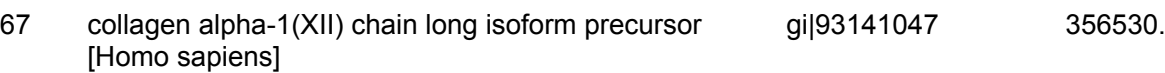

Peptide Information

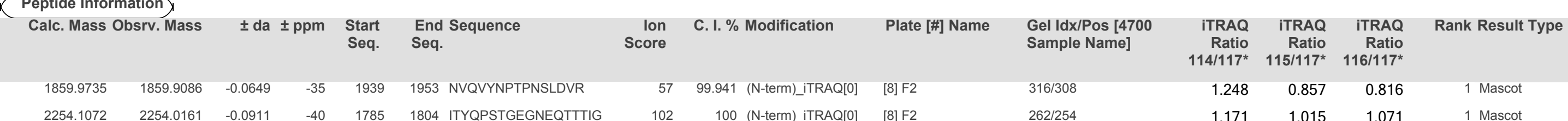

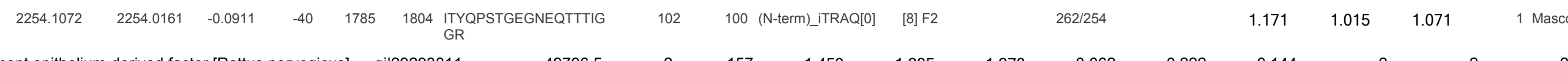

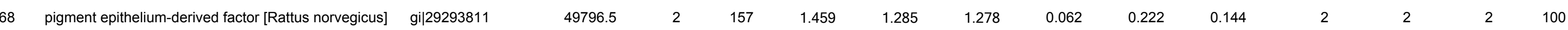
Peptide Information

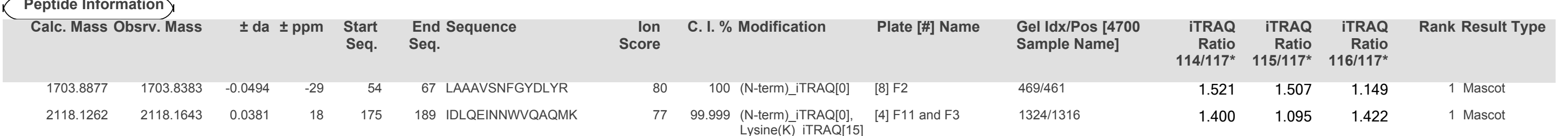

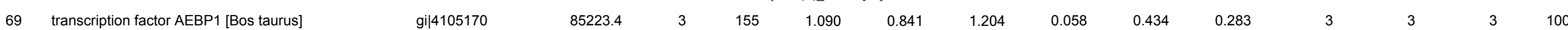

\section{Peptide Information}

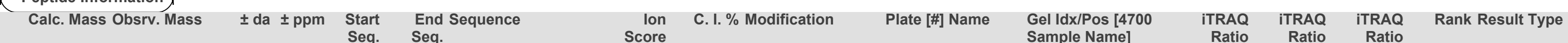

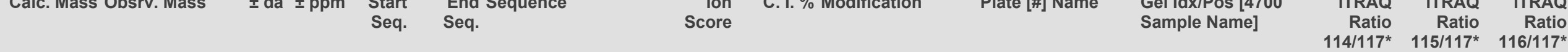

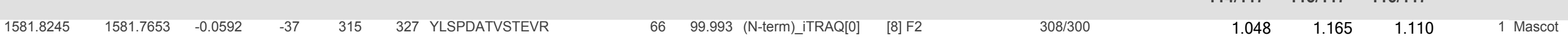

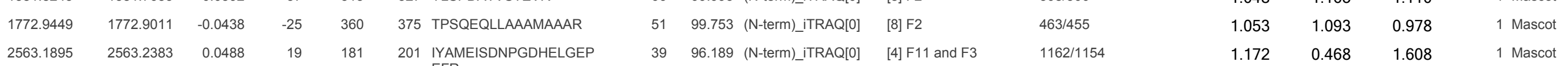

$$
\text { gi|3789962 } \quad 46124.2
$$

3155

$1.026 \quad 1.147 \quad 0.868$

$0.140 \quad 0.050$

0.130

Protein Group
$\begin{aligned} & \text { Chain A, The Crystal Structure Of Modified Bovine } \\ & \text { Fibrinogen (At } \sim 4 \text { Angstrom Resolution) }\end{aligned}$
gil6980814

Peptide Information

Calc. Mass Obsrv. Mass $\quad \pm$ da \pm ppm Start $\quad$ End Sequence 


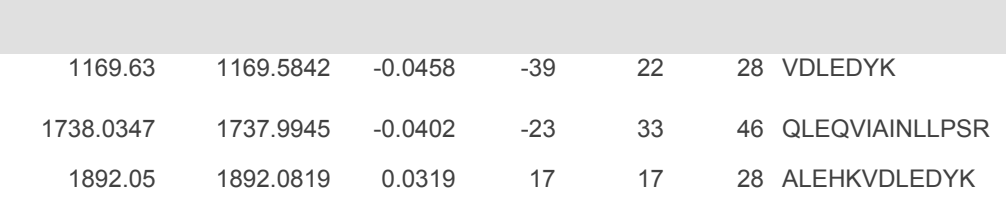

96.11 (N-term) - iTRAQ[0], $\quad[5]$ F5 and F10

$\begin{array}{lll}57 & 99.946 & (\mathrm{~N} \text {-term)_iTRAQ[0] } \\ {[8] \mathrm{F} 2}\end{array}$

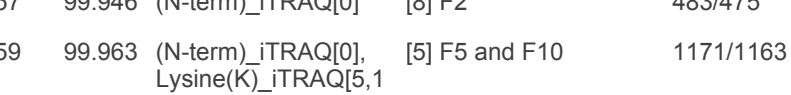
2] 0.5760 .514

71 haptoglobin heavy chain, $\mathrm{HpH}$ chain [dogs, Peptide, gil258499 30383

RecName: Full=Haptoglobin; Contains: RecName:

Peptide Information

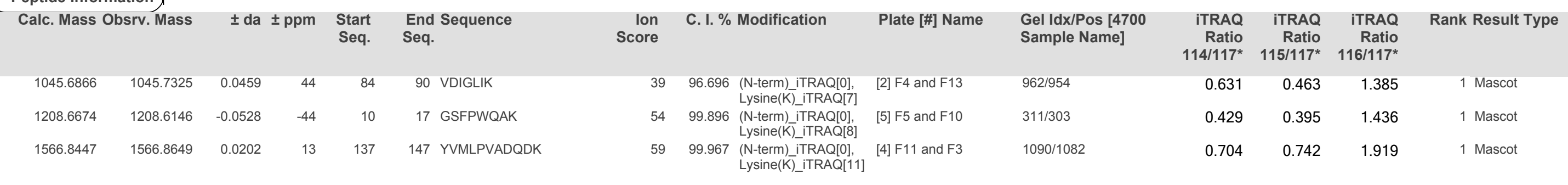

72 chromogranin B [Bos taurus]

\section{$0.997 \quad 1.128$}

1.328

0.094

Protein Group
RecName: Full=Secretogranin-1; AltName:

Full=Chromogranin-B;

gil12644006 $\quad 79151.7$

chromogranin B

gi|228903 $\quad 79374.9$

chromogranin B [Bos taurus]

gi|202

77524.9

secretogranin-1 [Bos taurus]

gi|30794308

79228.8

\section{Peptide Information}

\begin{tabular}{|c|c|c|c|c|c|c|c|c|c|c|c|c|c|}
\hline Calc. Mas: & srv. Mass & \pm da & ppm & $\begin{array}{l}\text { Start } \\
\text { Seq. }\end{array}$ & $\begin{array}{l}\text { End Sequence } \\
\text { Seq. }\end{array}$ & $\begin{array}{l}\text { lon } \\
\text { Score }\end{array}$ & C. I. \% Modification & Plate [\#] Name & $\begin{array}{l}\text { Gel Idx/Pos [4700 } \\
\text { Sample Name] }\end{array}$ & $\begin{array}{r}\text { iTRAQ } \\
\text { Ratio } \\
114 / 117^{*}\end{array}$ & $\begin{array}{r}\text { iTRAQ } \\
\text { Ratio } \\
115 / 117^{*}\end{array}$ & $\begin{array}{r}\text { iTRAQ } \\
\text { Ratio } \\
116 / 117^{*}\end{array}$ & Rank Result Type \\
\hline 2031.9868 & 2032.0377 & 0.0509 & 25 & 437 & 451 NYLDYGEEKGEEAAR & 44 & $\begin{array}{c}98.817 \begin{array}{l}\text { (N-term)_iTRAQ[0], } \\
\text { Lysine(K)_iTRAQ[9] }\end{array}\end{array}$ & [4] F11 and F3 & 1061/1053 & 1.091 & 1.196 & 1.300 & 1 Mascot \\
\hline 2445.0886 & 2444.9426 & -0.146 & -60 & 102 & 124 EDSGEGDAQVPTVADTE & 107 & 100 (N-term)_iTRAQ[0] & [5] F5 and F10 & 206/198 & 0.912 & 1.063 & 1.347 & Mascot \\
\hline
\end{tabular}

73 RecName: Full=Metalloproteinase inhibitor 2; AltName: gi|3915132 25216.4

$\begin{array}{llll}206 / 198 & \\ 2 & 149 & 0.770 & 0.150\end{array}$

25030.3

Chain C, Prommp-2TIMP-2 Complex

gi|22218677

RecName: Full=Metalloproteinase inhibitor 2; AltName: gi|135853

27530.7

RecName: Full=Metalloproteinase inhibitor 2; AltName: gi|267133
Full=Tissue inhibitor of metalloproteinases 2

27601

RecName: Full=Metalloproteinase inhibitor 2; AltName: gi|8478957
Full=Tissue inhibitor of metalloproteinases 2

TIMP-2 [Mus musculus]

gi|202054 27496.7

metalloproteinase inhibitor 2 [Cavia porcellus]

gi|290491246

27591.8

metalloproteinase inhibitor 2 precursor [Canis lupus $\quad$ gi|50978760 
tissue inhibitor of metalloproteinases-2 [Homo sapiens] gil1517893

\section{Peptide Information}

\begin{tabular}{|c|c|c|c|c|c|c|c|c|c|c|c|}
\hline Calc. Mass Obsrv. Mass & \pm da $\pm p p m$ & $\begin{array}{l}\text { Start } \\
\text { Seq. }\end{array}$ & $\begin{array}{l}\text { End Sequence } \\
\text { Seq. }\end{array}$ & $\begin{array}{l}\text { lon } \\
\text { Score }\end{array}$ & C. I. \% Modification & Plate [\#] Name & $\begin{array}{l}\text { Gel Idx/Pos [4700 } \\
\text { Sample Name] }\end{array}$ & $\begin{array}{r}\text { iTRAQ } \\
\text { Ratio } \\
114 / 117^{*}\end{array}$ & $\begin{array}{r}\text { iTRAQ } \\
\text { Ratio } \\
115 / 117^{*}\end{array}$ & $\begin{array}{r}\text { ITRAQ } \\
\text { Ratio } \\
116 / 117^{*}\end{array}$ & Rank Result Type \\
\hline 1915.0059 & 0.0227 & 182 & 196 GAAPPKQEFLDIEDP & 74 & 99.999 (N-term) -iTRAQ[0], & [4] F11 and F3 & $1114 / 1106$ & 0.644 & 0.798 & 0.856 & 1 Mascot \\
\hline 1965.0287 & 0.0504 & 30 & 44 EVDSGNDIYGNPIKR & 76 & 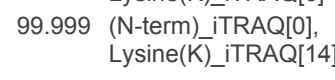 & {$[3] \mathrm{F} 14,15,16$ and $\mathrm{F} 6$} & $775 / 767$ & 0.920 & 0.926 & 1.011 & 1 Mascot \\
\hline ame: Full=Chitinas-3 & in 1. Altst & & 46168.8 & 3 & 1.195 & 1.256 & 0.200 & 0.281 & & 3 & 3 \\
\hline
\end{tabular}

RecName: Full=Chitinase-3-like protein 1; AltName: $\quad$ gi|55976543 46168.8

\section{Peptide Information}

\begin{tabular}{|c|c|c|c|c|c|c|c|c|c|c|c|c|c|}
\hline Calc. Mass 0 & srv. Mass & \pm da & ppm & $\begin{array}{l}\text { Start } \\
\text { Seq. }\end{array}$ & $\begin{array}{l}\text { End Sequence } \\
\text { Seq. }\end{array}$ & $\begin{array}{l}\text { lon } \\
\text { Score }\end{array}$ & C. I. \% Modification & Plate [\#] Name & $\begin{array}{l}\text { Gel Id dx/Pos [4700 } \\
\text { Sample Name] }\end{array}$ & $\begin{array}{r}\text { iTRAQ } \\
\text { Ratio } \\
114 / 117^{*}\end{array}$ & $\begin{array}{r}\text { iTRAQ } \\
\text { Ratio } \\
115 / 117^{*}\end{array}$ & $\begin{array}{r}\text { iTRAQ } \\
\text { Ratio } \\
116 / 117^{*}\end{array}$ & Rank Result Type \\
\hline 1537.8458 & 1537.7867 & -0.0591 & -38 & 272 & 286 TDVGAPISGPGIPGR & 66 & 99.994 (N-term)_iTRAQ[0] & [8] F2 & 287/279 & 1.488 & 0.683 & 1.670 & 1 Mascot \\
\hline 1676.8881 & 1676.8188 & -0.0693 & -41 & 92 & 105 TLLSVGGWNFGPER & 43 & 98.597 (N-term)_iTRAQ[0] & [8] F2 & $476 / 468$ & 1.083 & 1.039 & 1.113 & 1 Mascot \\
\hline 1961.9994 & 1961.9126 & -0.0868 & -44 & 129 & 144 THGFDGLDLAWLYPGR & 38 & 95.85 (N-term)_iTRAQ[0] & [5] F5 and F10 & $527 / 519$ & 1.060 & 1.134 & 1.066 & 1 Mascot \\
\hline
\end{tabular}

75 Tetranectin [Homo sapiens] $\quad$ gi|37409 25323.9

Peptide Information

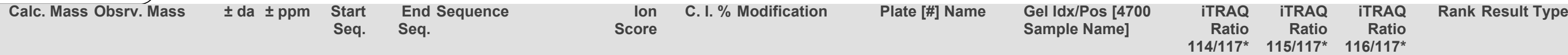

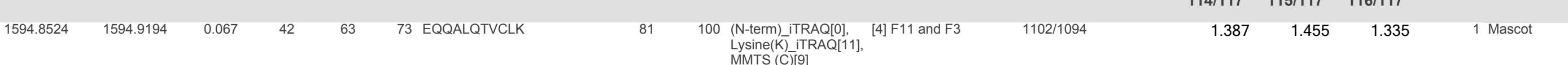

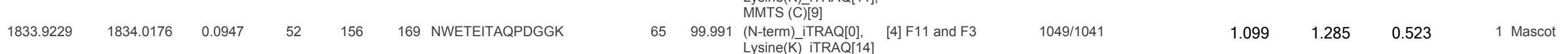

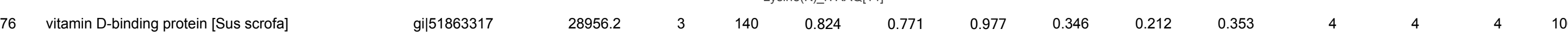

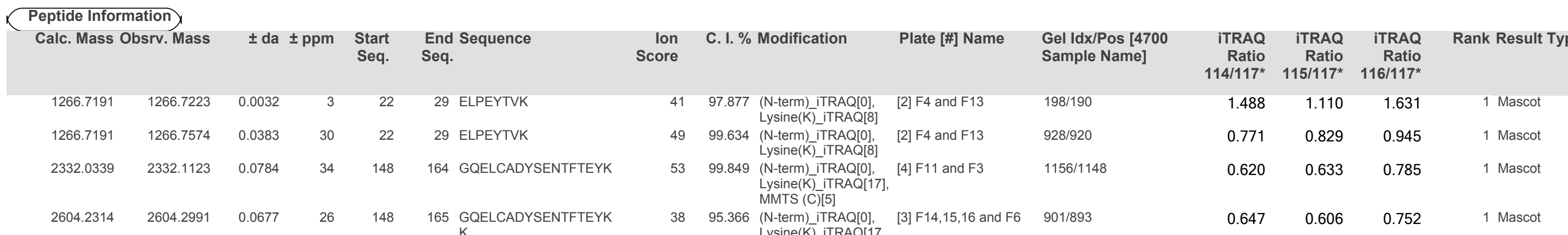

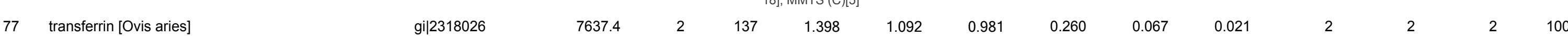

\begin{tabular}{|c|c|c|c|c|c|c|c|c|c|c|c|c|c|}
\hline \multicolumn{14}{|c|}{ Peptide Information } \\
\hline Calc. Mass & srv. Mass & \pm da & $\pm \mathrm{ppm}$ & $\begin{array}{l}\text { Start } \\
\text { Seq. }\end{array}$ & $\begin{array}{l}\text { End Sequence } \\
\text { Seq. }\end{array}$ & $\begin{array}{l}\text { lon } \\
\text { Score }\end{array}$ & C. I. \% Modification & Plate [\#] Name & $\begin{array}{l}\text { Gel Idx/Pos [4700 } \\
\text { Sample Name] }\end{array}$ & $\begin{array}{r}\text { iTRAQ } \\
\text { Ratio } \\
114 / 117^{*}\end{array}$ & $\begin{array}{r}\text { iTRAQ } \\
\text { Ratio } \\
115 / 117^{*}\end{array}$ & $\begin{array}{r}\text { iTRAQ } \\
\text { Ratio } \\
116 / 117^{*}\end{array}$ & Rank Result Type \\
\hline 1241.6075 & 1241.6476 & 0.0401 & 32 & 50 & 58 YYGYTGAFR & 38 & 95.945 (N-term)_iTRAQ[0] & [4] F11 and F3 & 1063/1055 & 1.179 & 1.029 & 1.001 & 1 Mascot \\
\hline 1824.7786 & 1824.7451 & -0.0335 & -18 & 9 & 23 FDEYFSAGCAPGSQR & 98 & $\begin{array}{l}100 \text { (N-term) iTRAQ[0], } \\
\text { MMTS (C) }[9]\end{array}$ & {$[8]$ F2 } & 399/391 & 1.659 & 1.159 & 0.961 & 1 Mascot \\
\hline jic system-as: & ted mer & & n precu & & 40308.9 & 2 & 1.118 & 1.027 & 0.108 & 0.096 & & 2 & 2 \\
\hline
\end{tabular}

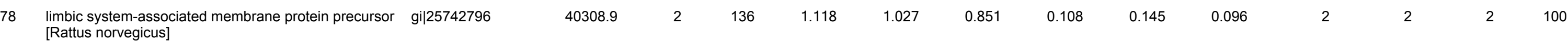

gi|1276899 $\quad 402929$ 
Peptide Information

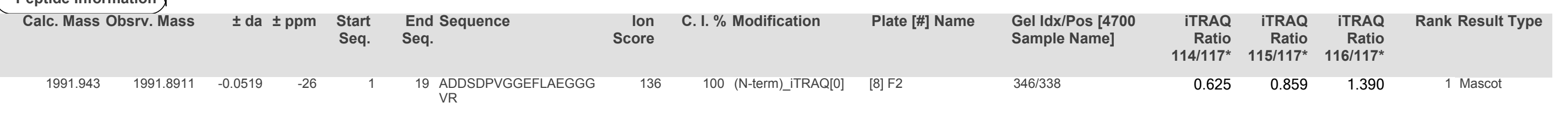

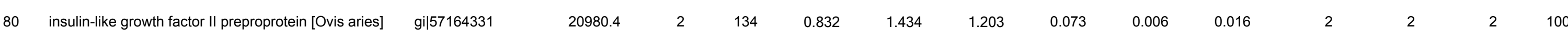
Protein Group $\quad$ gil552424 14279.7

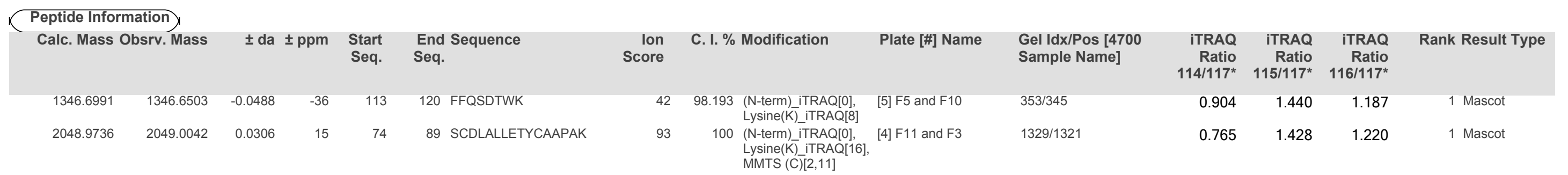

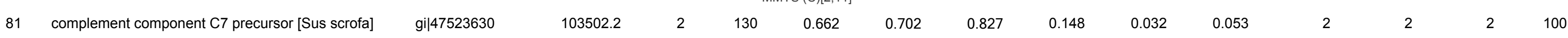

\begin{tabular}{|c|c|c|c|c|c|c|c|c|c|c|c|c|c|}
\hline Calc. Mass & srv. Mass & \pm da & ppm & $\begin{array}{l}\text { Start } \\
\text { Seq. }\end{array}$ & $\begin{array}{l}\text { End Sequence } \\
\text { Seq. }\end{array}$ & $\begin{array}{l}\text { lon } \\
\text { Score }\end{array}$ & C. I. \% Modification & Plate [\#] Name & $\begin{array}{l}\text { Gel Idx/Pos [4700 } \\
\text { Sample Name] }\end{array}$ & $\begin{array}{r}\text { iTRAQ } \\
\text { Ratio } \\
114 / 117^{*}\end{array}$ & $\begin{array}{r}\text { iTRAQ } \\
\text { Ratio } \\
115 / 117^{*}\end{array}$ & $\begin{array}{r}\text { iTRAQ } \\
\text { Ratio } \\
116 / 117^{*}\end{array}$ & Rank Result Ty \\
\hline 1656.7496 & 1656.7067 & -0.0429 & -26 & 812 & 824 EQTMTECEAGVLR & 55 & 99.918 (N-term)-iTRAQ[0], & [8] F2 & $361 / 353$ & 0.810 & 0.734 & 0.777 & 1 Mascot \\
\hline 2517.2871 & 2517.197 & -0.0901 & -36 & 295 & $314 \underset{Y K}{\text { LIDQYGTHYLQSGSLGGE }}$ & 75 & $\begin{array}{l}99.999 \\
\begin{array}{l}\text { N-term) iTRAQ[0], } \\
\text { Lysine(K)_TRAQ[2] }\end{array}\end{array}$ & [5] F5 and F10 & $390 / 382$ & 0.540 & 0.672 & 0.880 & 1 Mascot \\
\hline
\end{tabular}

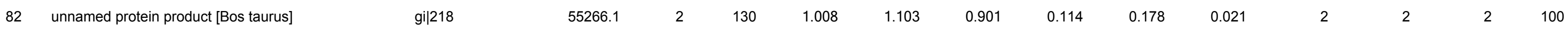
Protein Group 55166

53901.9

RecName: Full=Chromogranin-A; Short=CgA; Contains: gil116551
RecName: Full=Pancreastatin; Contains: RecName:

chromogranin A $\quad$ gi|225693 $\quad 55194$

chromogranin A [Bos taurus] $\quad$ gil163728 $\quad 55252.1$

chromogranin A [Bos taurus] $\quad$ gi|244424 55114

chromogranin-A [Bos taurus] $\quad$ gi|30794306 55182

Peptide Information 


\begin{tabular}{|c|c|c|c|c|c|c|c|c|c|c|c|c|c|}
\hline Calc. Mass & srv. Mass & $\pm \mathrm{da}$ & ppm & $\begin{array}{l}\text { Start } \\
\text { Seq. }\end{array}$ & $\begin{array}{l}\text { End Sequence } \\
\text { Seq. }\end{array}$ & $\begin{array}{l}\text { lon } \\
\text { Score }\end{array}$ & C. I. \% Modification & Plate [\#] Name & $\begin{array}{l}\text { Gel Idx/Pos [4700 } \\
\text { Sample Name] }\end{array}$ & $\begin{array}{r}\text { iTRAQ } \\
\text { Ratio } \\
114 / 117^{\star}\end{array}$ & $\begin{array}{r}\text { iTRAQ } \\
\text { Ratio } \\
115 / 117^{*}\end{array}$ & $\begin{array}{r}\text { iTRAQ } \\
\text { Ratio } \\
116 / 117^{*}\end{array}$ & Rank Result Type \\
\hline 2474.2871 & 2474.2 & -0.0871 & -35 & 420 & $\begin{array}{c}438 \underset{\mathrm{K}}{\mathrm{R}} \mathrm{R} \text { EDQELESLSAIEAELE } \\
\text {. }\end{array}$ & 69 & 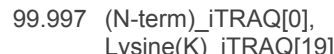 & [5] F5 and F10 & $488 / 480$ & 0.906 & 0.949 & 0.880 & $1 \mathrm{Mas}$ \\
\hline
\end{tabular}

83 dickkopf-related protein 3 precursor [Homo sapiens] $\quad$ gil $40548389 \quad 40483.6$

\begin{tabular}{lcc}
\hline Protein Group & gi|6049608 & 40384.6 \\
dickkopf-related protein 3 [Pongo abeli] & gi|197099502 & 40676 \\
hypothetical protein [Pongo abeli] & gi|l55730213 & 39809.3 \\
unnamed protein product [Homo sapiens] & gi|21749214 & 25207.3
\end{tabular}

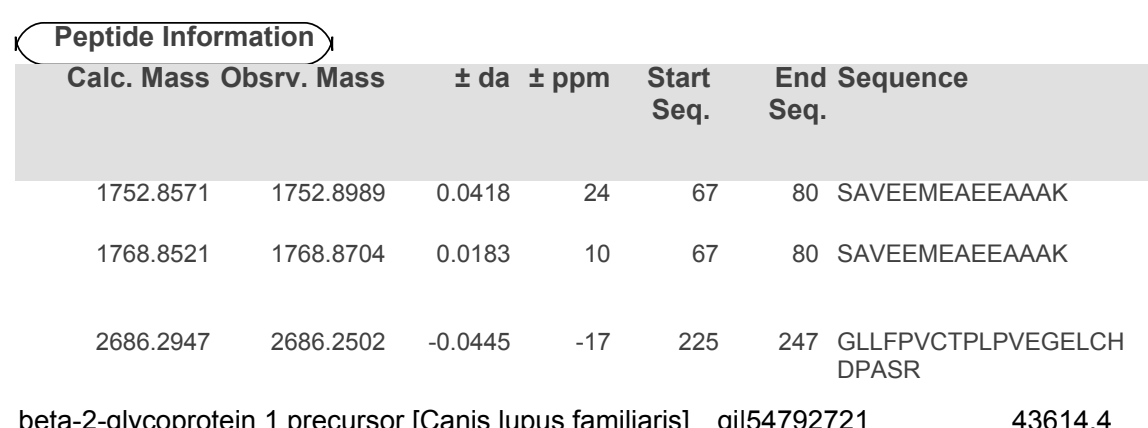

Ion C. I. \% Modification Plate [H] Name
Score

$\begin{array}{lrrr}\text { Gel Idx/Pos [4700 iTRAQ } & \text { iTRAQ } & \text { iTRAQ } & \text { Rank Result Type } \\ \text { Sample Name] } & \text { Ratio } & \text { Ratio } & \end{array}$ $\begin{array}{rrr}\text { Ratio } & \text { Ratio } & \text { Ratio } \\ 114 / 117^{*} & 115 / 117^{*} & 116 / 117^{\star}\end{array}$

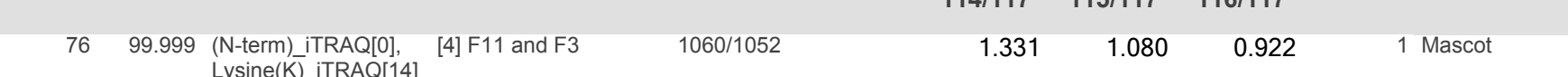

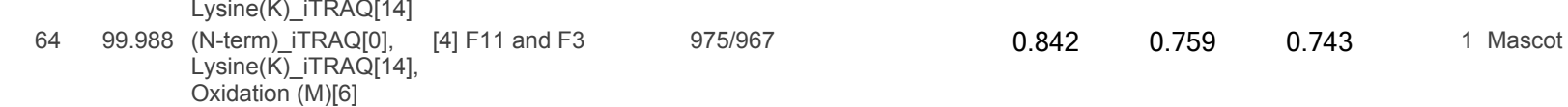

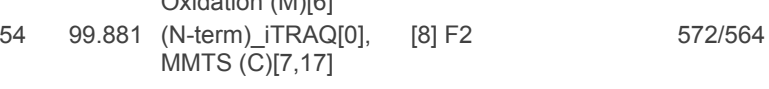

$\begin{array}{llll}0.505 & 0.916 \quad 0.583 \quad 1 \text { Mascot }\end{array}$

Peptide Information

\begin{tabular}{|c|c|c|c|c|c|c|c|c|c|c|c|c|c|c|}
\hline Calc. Mass & srv. Mass & \pm da & ppm & $\begin{array}{l}\text { Start } \\
\text { Seq. }\end{array}$ & $\begin{array}{l}\text { End Sequence } \\
\text { Seq. }\end{array}$ & $\begin{array}{l}\text { lon } \\
\text { Score }\end{array}$ & C. $1 . \%$ & Modification & Plate [\#] Name & $\begin{array}{l}\text { Gel Idx/Pos [4700 } \\
\text { Sample Name] }\end{array}$ & $\begin{array}{r}\text { iTRAQ } \\
\text { Ratio } \\
114 / 117^{*}\end{array}$ & $\begin{array}{r}\text { iTRAQ } \\
\text { Ratio } \\
115 / 117^{*}\end{array}$ & $\begin{array}{r}\text { iTRAQ } \\
\text { Ratio } \\
116 / 117^{*}\end{array}$ & Rank Result Type \\
\hline 1392.7522 & 1392.7346 & -0.0176 & -13 & 328 & 336 EHSSLAFWK & 64 & 99.99 & (N-term)_TRAQ[0], & [1] F8 061011 & $334 / 326$ & 0.492 & 0.622 & 1.012 & 1 Mascot \\
\hline 1592.8954 & 1592.8424 & -0.053 & -33 & 286 & 295 FKDGMLHGQK & 65 & 99.99 & $\begin{array}{l}\text { (N-term) iTRAQ[T], } \\
\text { Lysine(K)_iTRA[2, } \\
01\end{array}$ & [4] F11 and F3 & $120 / 112$ & 1.019 & 1.097 & 1.163 & 1 Mascot \\
\hline 1608.8904 & 1608.8298 & -0.0606 & -38 & 286 & 295 FKDGMLHGQK & 44 & 98.901 & 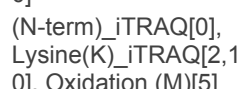 & [4] F11 and F3 & $120 / 112$ & 0.998 & 1.527 & 1.026 & 1 Mascot \\
\hline
\end{tabular}

$\begin{array}{llllllllllllll}85 & \text { WAP, kazal, immunoglobulin, kunitz and NTR } & \text { gi|267844918 } & 68543.1 & 2 & 128 & 0.728 & 0.965 & 0.937 & 0.179 & 0.043 & 0.000 & 2 & 2\end{array}$ domain-containing protein 2 precursor [Mus musculus]

Protein Group
Bikunin hlg [Homo sapiens]

PREDICTED: WAP, follistatin/kazal, immunoglobulin, $\quad$ gi|27675188 68579.3

kunitz and netrin domain containing 2 [Rattus no

$\begin{array}{lll}\text { WAP, kazal, immunoglobulin, kunitz and NTR } & \text { gil28212222 } & 69006.3 \\ \text { domain-containing protein }\end{array}$

growth and differentiation factor-associated serum $\quad$ gil32187114
protein 1 [Mus musculus]

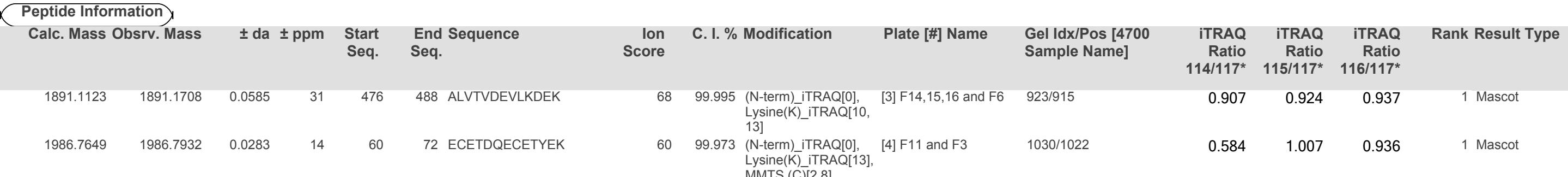

86 inter-alpha (globulin) inhibitor H4 (plasma $\quad$ gil59857769 $\quad 108789.4$

$125 \quad 0.952$

Kallkrein-sensitive glycoprotein) [Bos taurus] 


\begin{tabular}{|c|c|c|c|c|c|c|c|c|c|c|c|c|c|}
\hline Calc. Mass & srv. Mass & \pm da & ppm & $\begin{array}{l}\text { Start } \\
\text { Seq. }\end{array}$ & $\begin{array}{l}\text { End Sequence } \\
\text { Seq. }\end{array}$ & $\begin{array}{l}\text { Ion } \\
\text { Score }\end{array}$ & C. I. \% Modification & Plate [\#] Name & $\begin{array}{l}\text { Gel Idx/Pos [4700 } \\
\text { Sample Name] }\end{array}$ & $\begin{array}{r}\text { iTRAQ } \\
\text { Ratio } \\
114 / 117^{*}\end{array}$ & $\begin{array}{r}\text { iTRAQ } \\
\text { Ratio } \\
115 / 17^{*}\end{array}$ & $\begin{array}{r}\text { iTRAQ } \\
\text { Ratio } \\
116 / 117^{*}\end{array}$ & Rank Result Type \\
\hline 1636.9758 & 1636.933 & -0.0428 & -26 & 216 & 225 FKPTLSQQQK & 52 & 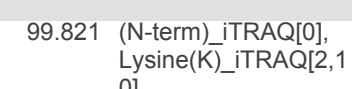 & [1] F8 061011 & 207/199 & 0.921 & 1.233 & 1.711 & 1 Mascot \\
\hline 2122.2244 & 2122.2793 & 0.0549 & 26 & 125 & 140 KTEQFQVSVSVAPAAK & 73 & 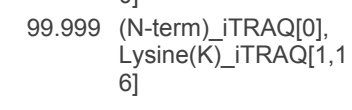 & {$[3] \mathrm{F} 14,15,16$ and $\mathrm{F} 6$} & $818 / 810$ & 0.984 & 1.090 & 1.445 & 1 Mascot \\
\hline
\end{tabular}

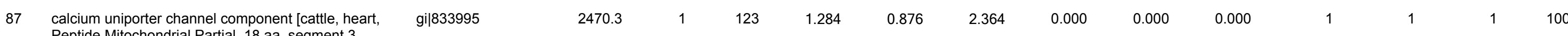
Peptide Information

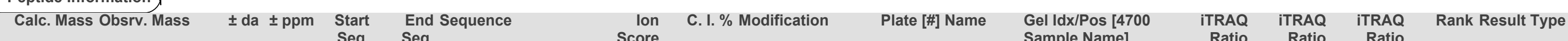
$\begin{array}{rrrr}\text { Ratio } & \text { Ratio } & \text { Ratio } \\ 114 / 117^{*} & 115 / 117^{*} & 116 / 117^{*}\end{array}$

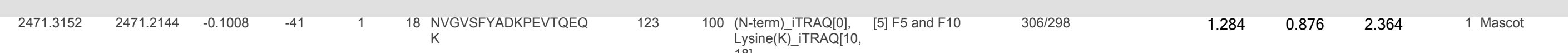

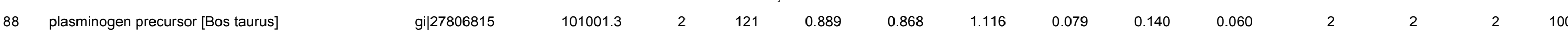

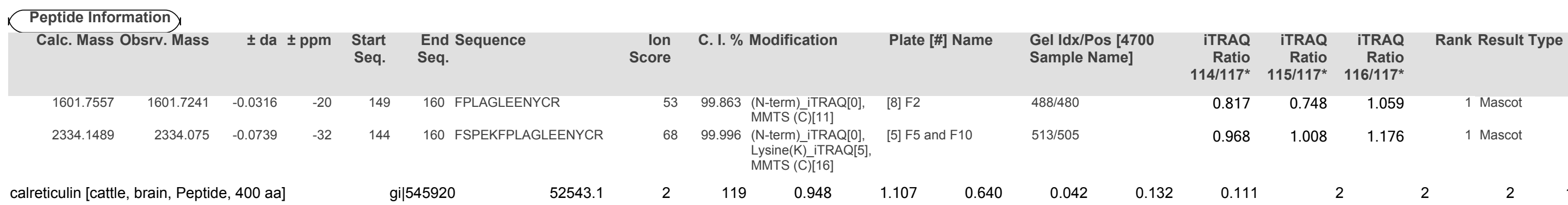

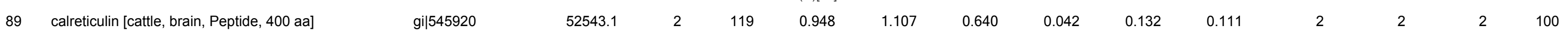
$\underset{\text { calreticulin precursor [Bos taurus] }}{\text { Pi|27806723 }} \quad 54199.1$

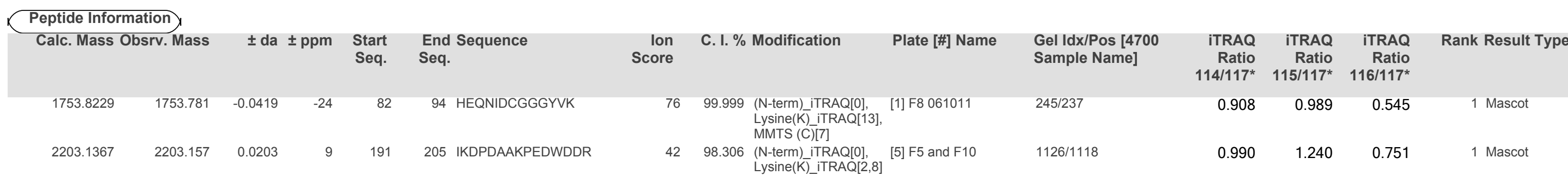

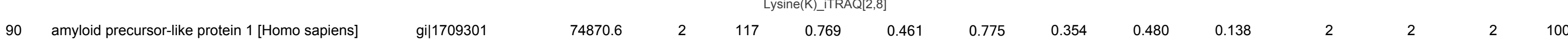
Parotein Group
sapied-like protein 1 isoform 2 precursor [Homo

Peptide Information

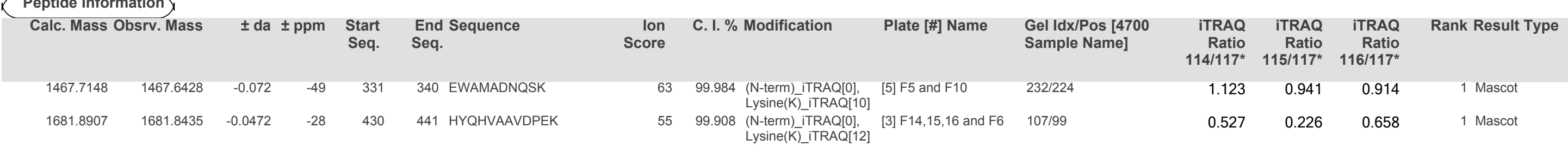

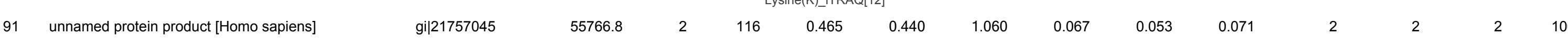

Protein Group

gi|138535 $\quad 57057.4$

RecName: Full=Vimentin $\quad$ gi|1353212 55177.6

VIM [Homo sapiens] $\quad$ gi|47115317 56907.4

unnamed protein product [Mus musculus] $\quad$ gi|l55291 56957.4 


$\begin{array}{llc}\text { vimentin [Bos taurus] } & \text { gi|289450 } & 57004.4 \\ \text { vimentin [Cricetulus griseus] } & \text { gi|860908 } & 47903.7 \\ \text { vimentin [Homo sapiens] } & \text { gi|62414289 } & 56979.4 \\ \text { vimentin [Homo sapiens] } & \text { gi|5030431 } & 44799.3 \\ \text { vimentin [Homo sapiens] } & \text { gi|340219 } & 57041.4 \\ \text { vimentin [Mus musculus] } & \text { gi|2078001 } & 54893.4 \\ \text { vimentin [Mus musculus] } & \text { gi|31982755 } & 57015.4 \\ \text { vimentin [Mus musculus] } & \text { gi|55408 } & 57049.4 \\ \text { vimentin [Pan troglodytes] } & \text { gi|57114172 } & 56975.4 \\ \text { vimentin [Rattus norvegicus] } & \text { gi|14389299 } & 57060.4 \\ \text { vimentin protein [Mus musculus] } & \text { gi|202368 } & 57001.4\end{array}$

\begin{tabular}{|c|c|c|c|c|c|c|c|c|c|c|c|}
\hline Calc. Mass Obsrv. Mass & $\pm \mathrm{da} \pm \mathrm{ppm}$ & $\begin{array}{l}\text { Start } \\
\text { Seq. }\end{array}$ & $\begin{array}{l}\text { End Sequence } \\
\text { Seq. }\end{array}$ & $\begin{array}{l}\text { lon } \\
\text { Score }\end{array}$ & C. I. \% Modification & Plate [\#] Name & $\begin{array}{l}\text { Gel Idx/Pos [4700 } \\
\text { Sample Name] }\end{array}$ & $\begin{array}{r}\text { iTRAQ } \\
\text { Ratio } \\
114 / 117^{*}\end{array}$ & $\begin{array}{r}\text { iTRAQ } \\
\text { Ratio } \\
115 / 117^{*}\end{array}$ & $\begin{array}{r}\text { iTRAQ } \\
\text { Ratio } \\
116 / 117^{*}\end{array}$ & Rank Result Type \\
\hline 1457.9188 & 0.0222 & 118 & 127 ILLAELEQLK & 43 & 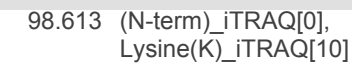 & ] [4] F11 and F3 & 1297/1289 & 0.407 & 0.393 & 1.131 & 1 Mascot \\
\hline 1980.8199 & -0.0821 & 439 & 454 DGQVINETSQHHDDLE & 73 & 99.998 (N-term)_iTRAQ[0] & [5] F5 and F10 & 217/209 & 0.532 & 0.493 & 0.994 & 1 Mascot \\
\hline
\end{tabular}

92 immunoglobulin kappa light chain constant region [Ovis gi|52367058 13395.7 $\begin{array}{ll}\text { Protein Group } & \\ \text { kappa light chain [Ovis aries] } & \text { gil147904806 }\end{array}$

\section{Peptide Information}

\begin{tabular}{|c|c|c|c|c|c|c|c|c|c|c|c|c|c|}
\hline Calc. Mass & siv. Mass & \pm da & \pm ppm & $\begin{array}{l}\text { Start } \\
\text { Seq. }\end{array}$ & $\begin{array}{l}\text { End Sequence } \\
\text { Seq. }\end{array}$ & $\begin{array}{l}\text { lon } \\
\text { Score }\end{array}$ & C. I. \% Modification & Plate [\#] Name & $\begin{array}{l}\text { Gel Idx/Pos [4700 } \\
\text { Sample Name] }\end{array}$ & $\begin{array}{r}\text { iTRAQ } \\
\text { Ratio } \\
114 / 117^{*}\end{array}$ & $\begin{array}{r}\text { iTRAQ } \\
\text { Ratio } \\
115 / 117^{*}\end{array}$ & $\begin{array}{r}\text { iTRAQ } \\
\text { Ratio } \\
116 / 117^{*}\end{array}$ & Rank Result Type \\
\hline 1116.7239 & 1116.7499 & 0.026 & 23 & 92 & 99 SLPTALVK & 39 & 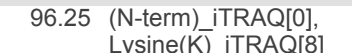 & [2] F4 and F13 & 944/936 & 1.171 & 0.939 & 0.989 & 1 Mascot \\
\hline 2366.2603 & 2366.1663 & -0.094 & -40 & 1 & 18 SDAQPSVFLFKPSEEQLR & 75 & 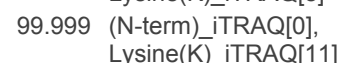 & [5] F5 and F10 & $401 / 393$ & 1.011 & 1.053 & 0.928 & 1 Mascot \\
\hline 2366.2603 & 2366.3062 & 0.0459 & 19 & 1 & 18 SDAQPSVFLFKPSEEQLR & 42 & $\begin{array}{l}98.068 \text { (N-term) iTRAQ[0], }] \\
\text { Lysine(K) iTRAQ[11] }\end{array}$ & [2] F4 and F13 & $227 / 219$ & 1.698 & 1.114 & 0.879 & 1 Mascot \\
\hline
\end{tabular}

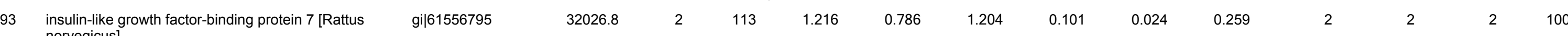
(Pervegicus]

\section{Peptide Information}

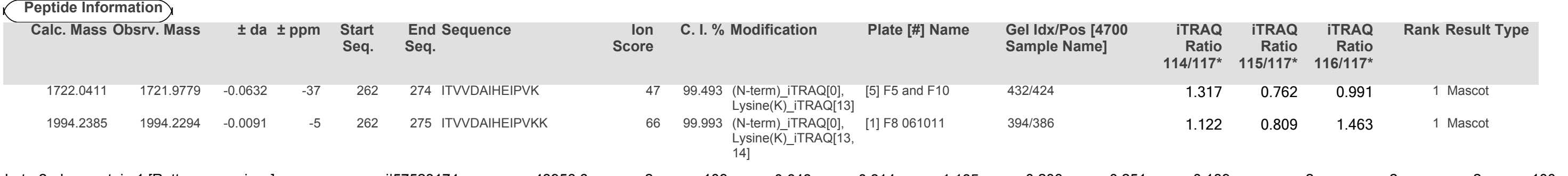

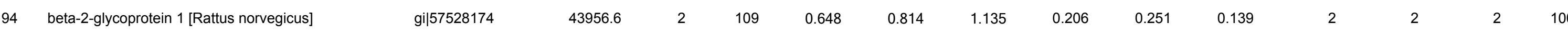

Peptide Information

Calc. Mass Obsrv. Mass $\quad \pm$ da \pm ppm Start $\quad$ End Sequence

Ion
Score

Plate [H] Name

Gel Idx/Pos [4700 iTRAQ iTRAQ iTRAQ Rank Result Type
} 


\begin{tabular}{|c|c|c|c|c|c|c|c|c|c|c|c|c|c|c|c|c|c|}
\hline & & & & & & & & & & & & & $114 / 117^{\star}$ & $115 / 117^{*}$ & $116 / 117^{*}$ & & \\
\hline 1392.7522 & 1392.7346 & -0.0176 & -13 & 328 & \multirow{2}{*}{$\begin{array}{l}336 \text { EHSSLAFWK } \\
78 \text { FTCPLTGMWPINTLK }\end{array}$} & 64 & 99.99 & \multirow{2}{*}{ 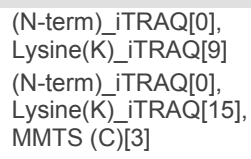 } & \multirow{2}{*}{\multicolumn{2}{|c|}{$\begin{array}{l}{[1] \text { F8 } 061011} \\
{[4] F 11 \text { and F3 }}\end{array}$}} & \multirow{2}{*}{\multicolumn{2}{|c|}{$\begin{array}{l}334 / 326 \\
1359 / 1351\end{array}$}} & 0.492 & 0.622 & \multirow{2}{*}{$\begin{array}{l}1.012 \\
1.274\end{array}$} & \multicolumn{2}{|l|}{1 Mascot } \\
\hline 2056.0662 & 2056.115 & 0.0488 & 24 & 64 & & 44 & 98.943 & & & & & & 0.854 & 1.065 & & 1 Mascot & \\
\hline \multicolumn{4}{|c|}{ ecName: Full=Superoxide dismutase [Cu-Zn] } & & |75061021 & 2 & 106 & 1.105 & 0.962 & 0.926 & 0.052 & 0.073 & 0.095 & & 2 & 2 & 100 \\
\hline \multicolumn{18}{|c|}{ Peptide Information } \\
\hline \multicolumn{2}{|c|}{ Calc. Mass Obsrv. Mass } & \pm da \pm & $\pm \mathrm{ppm}$ & $\begin{array}{l}\text { Start } \\
\text { Seq. }\end{array}$ & $\begin{array}{l}\text { End Sequence } \\
\text { Seq. }\end{array}$ & $\begin{array}{l}\text { lon } \\
\text { Score }\end{array}$ & \multicolumn{2}{|c|}{ C. I. \% Modification } & \multicolumn{2}{|c|}{ Plate [\#] Name } & \multicolumn{2}{|c|}{$\begin{array}{l}\text { Gel Idx/Pos [4700 } \\
\text { Sample Name] }\end{array}$} & $\begin{array}{r}\text { ITRAQ } \\
\text { Ratio } \\
114 / 117^{*}\end{array}$ & $\begin{array}{r}\text { iTRAQ } \\
\text { Ratio } \\
115 / 117^{*}\end{array}$ & $\begin{array}{r}\text { iTRAQ } \\
\text { Ratio } \\
116 / 117^{*}\end{array}$ & Rank Result Type & \\
\hline 1226.7103 & 1226.7273 & 0.017 & 14 & 79 & \multirow{2}{*}{$\begin{array}{l}87 \text { HVGDLGNVK } \\
127 \text { TMVVHEKPDDLGR }\end{array}$} & 50 & 99.733 & \multirow{2}{*}{ 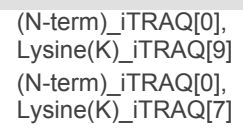 } & \multirow{2}{*}{\multicolumn{2}{|c|}{$\begin{array}{l}{[7] \mathrm{F} 12 \text { and } \mathrm{F9} \text { and F7 }} \\
\text { attempt } 2 \\
{[7] \mathrm{F} 12 \text { and } \mathrm{F9} \text { and F7 }} \\
\text { attempt } 2 \text { ? }\end{array}$}} & $828 / 820$ & & 1.055 & 0.894 & 0.839 & 1 Mascot & \\
\hline 1784.9575 & 1785.0632 & 0.1057 & 59 & 115 & & 56 & 99.928 & & & & $854 / 846$ & & 1.157 & 1.035 & 1.021 & 1 Mascot & \\
\hline $\begin{array}{l}\text { Imunoglobulin } \\
\text { Jvis aries] }\end{array}$ & bda-6c ligl & ain vari & able reg & & 12870.2 & 1 & 106 & 1.705 & 0.934 & 1.258 & 0.000 & 0.000 & 0.000 & & 1 & 1 & 100 \\
\hline
\end{tabular}

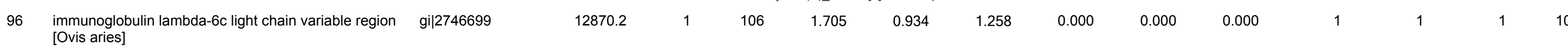

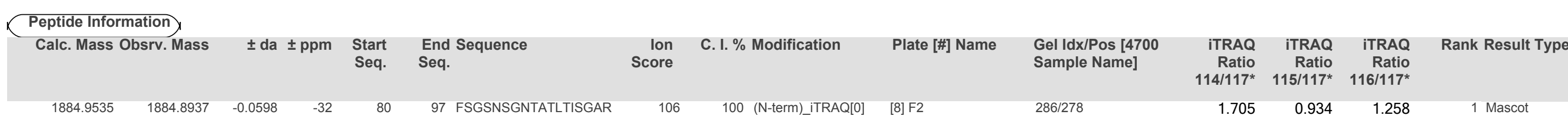

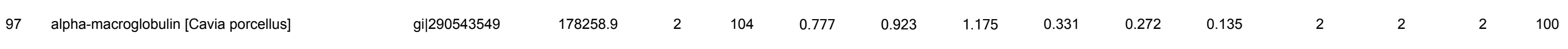

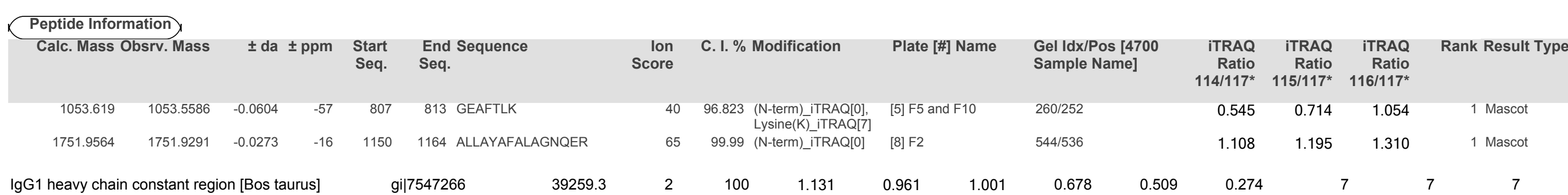

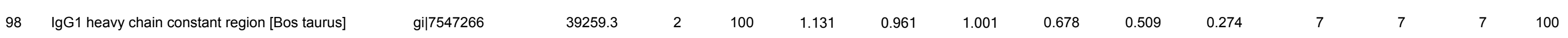
Protein Group

\begin{tabular}{|c|c|c|c|c|c|c|c|c|c|c|c|c|c|c|}
\hline \multicolumn{2}{|c|}{ Calc. Mass Obsrv. Mass } & \multicolumn{2}{|c|}{ \pm da $\pm p p m$} & \multirow{2}{*}{$\begin{array}{l}\begin{array}{l}\text { Start } \\
\text { Seq. }\end{array} \\
203\end{array}$} & \multirow{2}{*}{$\begin{array}{l}\text { End Sequence } \\
\text { Seq. } \\
214 \text { VHNEGLPAPIVR }\end{array}$} & \multirow{2}{*}{$\begin{array}{r}\begin{array}{r}\text { lon } \\
\text { Score }\end{array} \\
43\end{array}$} & \multirow{2}{*}{\multicolumn{2}{|c|}{$\begin{array}{l}\text { C. I. \% Modification } \\
98.568 \text { (N-term)_iTRAQ[0] }\end{array}$}} & \multirow{2}{*}{$\begin{array}{l}\text { Plate [\#] Name } \\
\text { [4] F11 and F3 }\end{array}$} & \multirow{2}{*}{$\begin{array}{l}\text { Gel Idx/Pos [4700 } \\
\text { Sample Name] } \\
197 / 189\end{array}$} & \multirow{2}{*}{$\begin{array}{r}\text { iTRAQ } \\
\text { Ratio } \\
114 / 117^{*} \\
2.222\end{array}$} & \multirow{2}{*}{$\begin{array}{r}\text { iTRAQ } \\
\text { Ratio } \\
115 / 117^{*} \\
1.945\end{array}$} & \multirow{2}{*}{$\begin{array}{r}\text { iTRAQ } \\
\text { Ratio } \\
116 / 117^{*} \\
1.440\end{array}$} & \multirow{2}{*}{$\begin{array}{c}\text { Rank Result Type } \\
1 \text { Mascot }\end{array}$} \\
\hline 1445.8348 & 1445.7682 & -0.0666 & -46 & & & & & & & & & & & \\
\hline 1445.8348 & 1445.7845 & -0.0503 & -35 & 203 & 214 VHNEGLPAPIVR & 43 & 98.545 & (N-term)_iTRAQ[0] & {$[3] \mathrm{F} 14,15,16$ and $F 6$} & $167 / 159$ & 1.642 & 1.315 & 1.330 & 1 Mascot \\
\hline 1445.8348 & 1445.8787 & 0.0439 & 30 & 203 & 214 VHNEGLPAPIVR & 42 & 98.077 & (N-term)_iTRAQ[0] & {$[3] \mathrm{F} 14,15,16$ and $F 6$} & $794 / 786$ & 1.707 & 1.419 & 1.169 & 1 Mascot \\
\hline 2115.1584 & 2115.1035 & -0.0549 & -26 & 225 & 240 EPQVYVLAPPQEELSK & 55 & 99.91 & (N-term) iTRAQ[0], & [5] F5 and F10 & $378 / 370$ & 0.547 & 0.633 & 0.801 & 1 Mascot \\
\hline 2115.1584 & 2115.1072 & -0.0512 & -24 & 225 & 240 EPQVYVLAPPQEELSK & 54 & 99.885 & 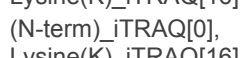 & [8] F2 & $379 / 371$ & 0.803 & 0.626 & 0.816 & 1 Mascot \\
\hline 2115.1584 & 2115.1665 & 0.0081 & 4 & 225 & 240 EPQVYVLAPPQEELSK & 58 & 99.95 & (N-term) iTRAQ[O], & [4] F11 and F3 & $1120 / 1112$ & 0.783 & 0.742 & 0.887 & 1 Mascot \\
\hline 2115.1584 & 2115.2253 & 0.0669 & 32 & 225 & 240 EPQVYVLAPPQEELSK & 40 & 96.903 & $\begin{array}{l}\text { (N-term) iTRAQ[0], } \\
\text { Lysine(K)_iTRAQ[16] }\end{array}$ & [4] F11 and F3 & $1286 / 1278$ & 1.105 & 0.710 & 0.773 & 1 Mascot \\
\hline
\end{tabular}

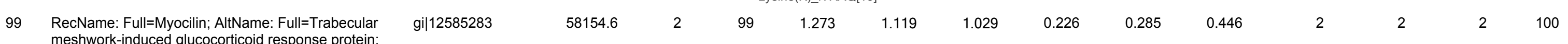
Protein Group
myocilin [Bos taurus]

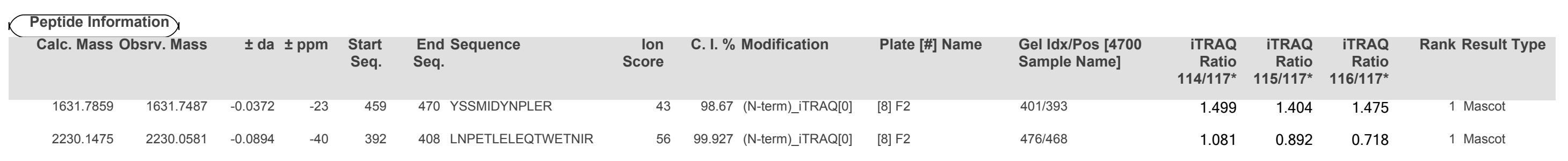


100 immunoglobulin mu chain [Ovis aries]

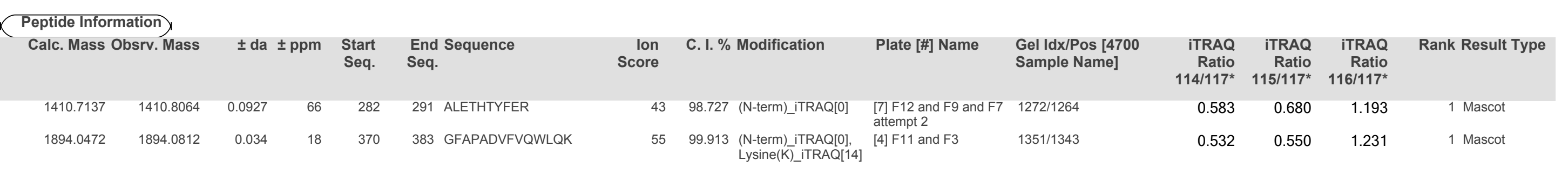

101 Chain C, The Crystal Structure Of Modified Bovine $\quad$ gil6980816 51472.4

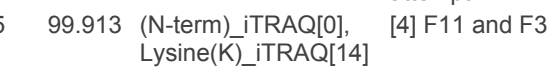

$1351 / 1343$

$\begin{array}{lll}0.532 & 0.550 \quad 1.23\end{array}$

Peptide Information

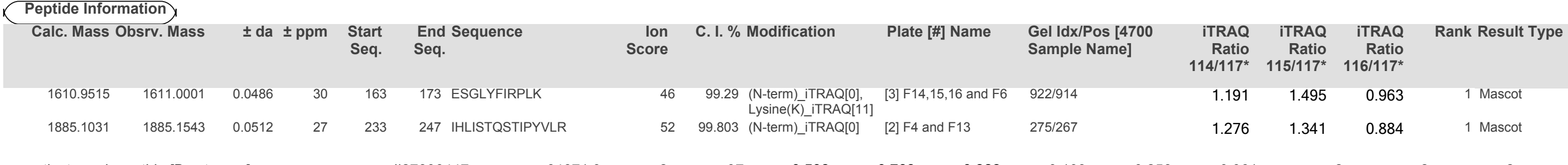

102 proactivator polypeptide [Bos taurus]

gi|27806447 $\quad 64671.9$

Peptide Information

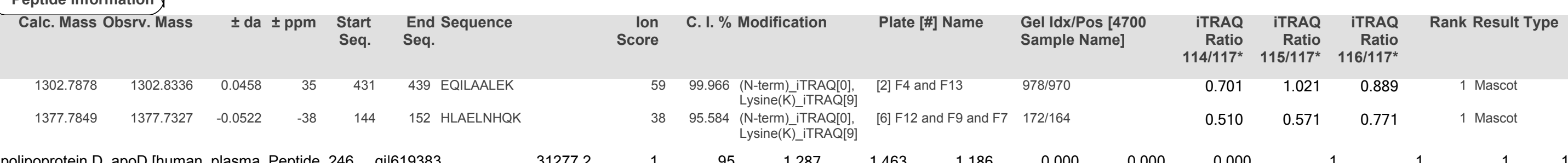

$103 \begin{aligned} & \text { apolipoprotein D, apoD [human, plasma, Peptide, } 246 \quad \text { gil619383 } \\ & \text { aa] }\end{aligned}$

Protein Group

apolipoprotein D precursor [Homo sapiens] $\quad$ gil4502163 23220.9

Peptide Information

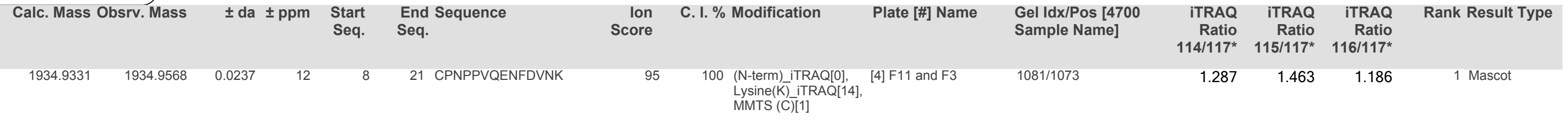

104 insulin-like growth factor-binding protein 6 [Bos taurus] gi|5705897

14741.4

$93 \quad 0.793$

Protein Group

RecName: Full=Insulin-like growth factor-binding protein gi|1352416 26986.4
6: Short=IBP-6; Short=|GF-binding protein

insulin-like growth factor binding protein 6 [Bos taurus] gi|28630915

9734.6

insulin-like growth factor binding protein 6 [OVis aries] $\quad$ gi|12061034 9071.5

insulin-like growth factor-binding protein 6 precursor $\quad$ gil168693654 26930.4

[Mus musculus]

insulin-like growth factor-binding protein 6 precursor $\quad$ gil6981090 25686
[Rattus norvegicus]

Peptide Information

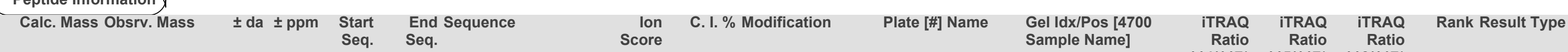




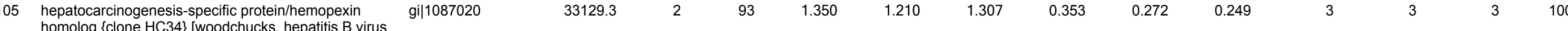
Peptide Information

\begin{tabular}{|c|c|c|c|c|c|c|c|c|c|c|c|c|c|}
\hline \multirow{2}{*}{\multicolumn{2}{|c|}{$\begin{array}{l}\text { Peptide Information } \\
\text { Calc. Mass Obsrv. Mass }\end{array}$}} & \multirow{2}{*}{\multicolumn{2}{|c|}{ \pm da \pm ppm }} & \multirow[b]{2}{*}{$\begin{array}{l}\text { Start } \\
\text { Seq. }\end{array}$} & \multirow[b]{2}{*}{$\begin{array}{l}\text { End Sequence } \\
\text { Seq. }\end{array}$} & \multirow[b]{2}{*}{$\begin{array}{l}\text { lon } \\
\text { Score }\end{array}$} & \multirow{2}{*}{ C. I. \% Modification } & \multirow[b]{2}{*}{ Plate [\#] Name } & \multirow[b]{2}{*}{$\begin{array}{l}\text { Gel Id dx/Pos [4700 } \\
\text { Sample Name] }\end{array}$} & \multirow[b]{2}{*}{$\begin{array}{r}\text { iTRAQ } \\
\text { Ratio } \\
114 / 117^{*}\end{array}$} & \multirow[b]{2}{*}{$\begin{array}{r}\text { iTRAQ } \\
\text { Ratio } \\
115 / 117^{*}\end{array}$} & \multirow[b]{2}{*}{$\begin{array}{r}\text { iTRAQ } \\
\text { Ratio } \\
116 / 117^{*}\end{array}$} & \multirow[b]{2}{*}{ Rank Result Type } \\
\hline & & & & & & & & & & & & & \\
\hline 1261.7555 & 1261.7815 & 0.026 & 21 & 196 & 202 LWWLDLK & 43 & $\begin{array}{c}98.538 \\
\begin{array}{l}\text { N-term) iTRAQ[0], } \\
\text { Lysine(K) iTRAQ[7] }\end{array}\end{array}$ & [2] F4 and F13 & $374 / 366$ & 1.009 & 0.913 & 1.217 & 1 Mascot \\
\hline 1261.7555 & 1261.796 & 0.0405 & 32 & 196 & 202 LWWLDLK & 39 & 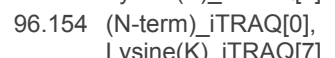 & [2] F4 and F13 & 1045/1037 & 1.368 & 1.335 & 1.103 & 1 Mascot \\
\hline
\end{tabular}

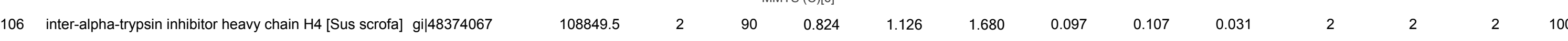

\begin{tabular}{|c|c|c|c|c|c|c|c|c|c|c|c|c|c|}
\hline \multicolumn{14}{|c|}{ Peptide Information } \\
\hline Calc. Mass & srv. Mass & $\begin{array}{l} \pm \mathrm{da} \\
\end{array}$ & ppm & $\begin{array}{l}\text { Start } \\
\text { Seq. }\end{array}$ & $\begin{array}{l}\text { End Sequence } \\
\text { Seq. }\end{array}$ & $\begin{array}{l}\text { Ion } \\
\text { Score }\end{array}$ & C. I. \% Modification & Plate [\#] Name & $\begin{array}{l}\text { Gel Idx/Pos [4700 } \\
\text { Sample Name] }\end{array}$ & $\begin{array}{r}\text { iTRAQ } \\
\text { Ratio } \\
114 / 117^{*}\end{array}$ & $\begin{array}{r}\text { iTRAQ } \\
\text { Ratio } \\
115 / 117^{*}\end{array}$ & $\begin{array}{r}\text { iTRAQ } \\
\text { Ratio } \\
116 / 117^{*}\end{array}$ & Rank Result Type \\
\hline 1373.6796 & 1373.7531 & 0.0735 & 54 & 534 & 542 YIFHSFMER & 38 & 95.822 (N-term)_iTRAQ[0] & 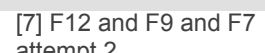 & 1391/1383 & 0.737 & 1.029 & 1.650 & 1 Mascot \\
\hline 1636.9758 & 1636.933 & -0.0428 & -26 & 213 & 222 FKPTLSQQQK & 52 & $\begin{array}{c}99.821 \\
\text { (N-term)_iTRAQ[0], } \\
\text { Lysine(K)_iTRAQ[2,1 } \\
0]\end{array}$ & [1] F8 061011 & 207/199 & 0.921 & 1.233 & 1.711 & 1 Mascot \\
\hline
\end{tabular}

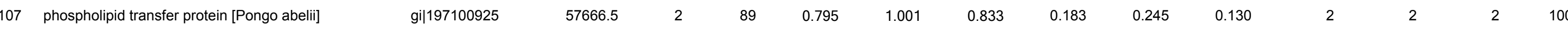

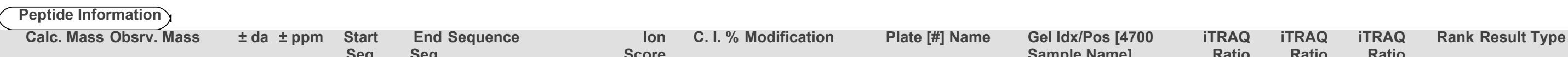
$\begin{array}{crrr}\text { Ratio } & \text { Ratio } & \text { Ratio } \\ 114 / 117^{*} & 115 / 117^{*} & 116 / 117^{*}\end{array}$

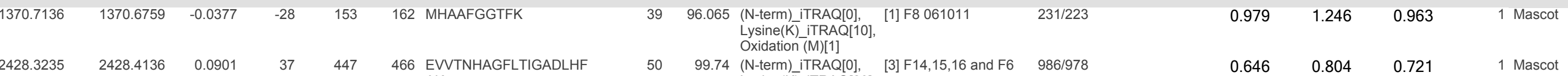

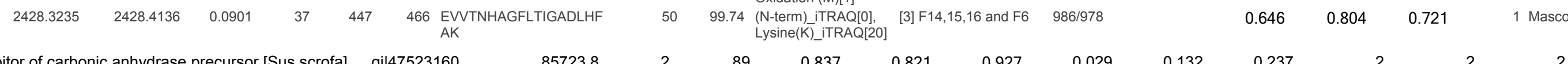

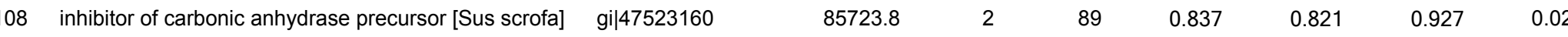

Peptide Information

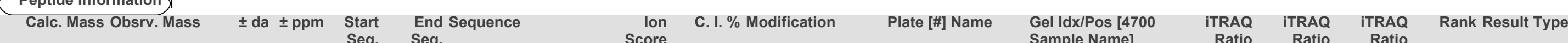

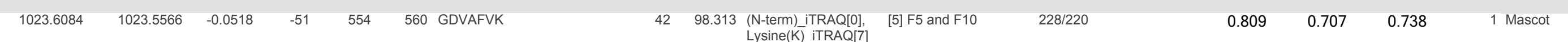

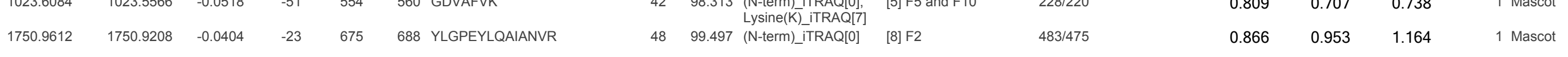

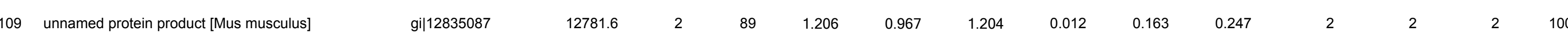

Protein Group $\quad$ gil42734496 27992.8

Fam3c protein [Mus musculus] $\quad$ gil33417025 $\quad 28022.8$

Fam3c protein [Mus musculus] $\quad$ gi|47125071 36097

PREDICTED: protein FAM3C-like isoform 2 [Pan $\quad$ gil55629272 27901.6

troglodytes

family with sequence similarity 3, member C [Rattus $\quad$ gil38454280 $\quad 27953.7$

family with sequence similarity 3 , member $\mathrm{C}$ precursor gi|7661714 27920.7
[Homo sapiens]

Peptide Information

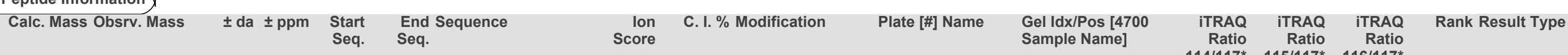

Seq. Seq. Score Sample Name] $\begin{gathered}\text { Ratio } \\ 114 / 17^{*} \\ 115 / 117^{*}\end{gathered} \quad \begin{gathered}\text { Ratio } \\ 116 / 117^{*}\end{gathered}$


$\begin{array}{llllll}1359.6416 & 1359.5864 & -0.0552 & -41 & 54 & 62 \\ \text { DNWVFCGGK }\end{array}$

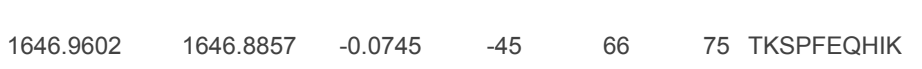

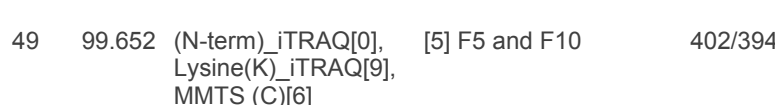

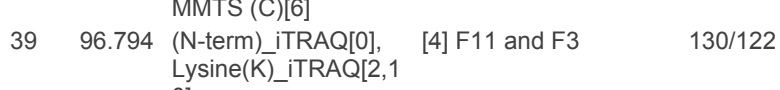

$\begin{array}{llll}1.218 & 1.130 & 1.451 & 1 \text { Mascot }\end{array}$

$\begin{array}{llll}1.194 & 0.828 & 0.999 & 1 \text { Mascot }\end{array}$

110 collagen type I alpha 1 [Sus scrofa]

gi|37811669 $\quad 10249$

$\begin{array}{llll}87 & 1.328 & 2.093 & 1.125\end{array}$

$0.000 \quad 0.000$

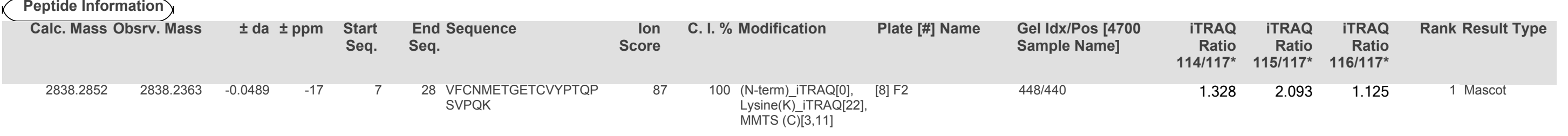

111 contactin-2 precursor [Homo sapiens]

Protein Group

gi|4827022 $\quad 119638.3$

contactin-2 [Pongo abelii

gi 444868

119652.4

gil197098330 $\quad 119769.4$

\section{Peptide Information}

\begin{tabular}{|c|c|c|c|c|c|c|c|c|c|c|c|c|c|}
\hline Calc. Mass & srv. Mass & \pm da & ppm & $\begin{array}{l}\text { Start } \\
\text { Seq. }\end{array}$ & $\begin{array}{l}\text { End Sequence } \\
\text { Seq. }\end{array}$ & $\begin{array}{l}\text { lon } \\
\text { Score }\end{array}$ & C. I. \% Modification & Plate [\#] Name & $\begin{array}{l}\text { Gell Idx/Pos [4700 } \\
\text { Sample Name] }\end{array}$ & $\begin{array}{r}\text { iTRAQ } \\
\text { Ratio } \\
114 / 117^{*}\end{array}$ & $\begin{array}{r}\text { iTRAQ } \\
\text { Ratio } \\
115 / 117^{*}\end{array}$ & $\begin{array}{r}\text { iTRAQ } \\
\text { Ratio } \\
116 / 117^{*}\end{array}$ & Rank Result Type \\
\hline 1390.7616 & 1390.785 & 0.0234 & 17 & 129 & 137 FGFLQEFSK & 48 & 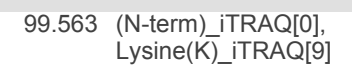 & [2] F4 and F13 & 296/288 & 1.199 & 0.771 & 0.765 & 1 Mascot \\
\hline 1428.8546 & 1428.8223 & -0.0323 & -23 & 465 & 476 VTVTPDGTLIIR & 38 & 95.973 (N-term)_TRAQ[0] & [8] F2 & 375/367 & 1.310 & 0.688 & 0.784 & 1 Mascot \\
\hline
\end{tabular}

112 RecName: Full=Fibulin-1; Short=FIBL-1

Protein Group

gi|30580425 70865.5

$2 \quad 85$

$0.988 \quad 0.598$

0.215

$\begin{array}{llll}0.688 & 0.784 & 1 \text { Masco }\end{array}$

gi|18490682 $\quad 80422.8$

fibulin-1 C [Homo sapiens]

gi|31419 $\quad 80474.9$

fibulin-1 isoform C precursor [Homo sapiens]

gi|34734062 $\quad 80460.9$

Peptide Information

\begin{tabular}{|c|c|c|c|c|c|c|c|c|c|c|c|c|c|c|c|c|c|}
\hline Calc. Mass Obsrv. Mass & \pm da \pm & opm & $\begin{array}{l}\text { Start } \\
\text { Seq. }\end{array}$ & \multicolumn{2}{|c|}{$\begin{array}{l}\text { End Sequence } \\
\text { Seq. }\end{array}$} & $\begin{array}{l}\text { lon } \\
\text { Score }\end{array}$ & \multicolumn{2}{|c|}{ C. I. \% Modification } & \multicolumn{2}{|c|}{ Plate [\#] Name } & \multicolumn{2}{|c|}{$\begin{array}{l}\text { Gel Idx/Pos [4700 } \\
\text { Sample Name] }\end{array}$} & $\begin{array}{r}\text { iTRAQ } \\
\text { Ratio } \\
114 / 117^{*}\end{array}$ & $\begin{array}{r}\text { iTRAQ } \\
\text { Ratio } \\
115 / 117^{*}\end{array}$ & $\begin{array}{r}\text { iTRAQ } \\
\text { Ratio } \\
116 / 117^{*}\end{array}$ & \multicolumn{2}{|l|}{ Rank Result Type } \\
\hline 1320.7183 & -0.0339 & -26 & 580 & \multicolumn{2}{|c|}{589 HGTVSSFVAK } & 41 & 97.857 & 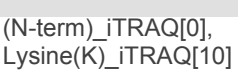 & \multirow{2}{*}{\multicolumn{2}{|c|}{$\begin{array}{l}\text { [1] F8 } 061011 \\
{[8] \text { F2 }}\end{array}$}} & 213/205 & & 0.829 & 0.879 & 0.749 & 1 Mascot & \\
\hline 1580.6099 & -0.0352 & -22 & 466 & 476 CLA & ENYR & 44 & 98.755 & $\begin{array}{l}\text { (N-term)iTRAQ[0], } \\
\text { MMTS (C))[1,6] }\end{array}$ & & & $463 / 455$ & & 1.829 & 1.274 & 1.304 & 1 Mascot & \\
\hline \multicolumn{3}{|c|}{ dherin-15 preproprotein [Homo sapiens] } & & 14826669 & 91488.9 & 1 & 84 & 1.394 & 0.891 & 0.719 & 0.000 & 0.000 & 0.000 & & 1 & 1 & 100 \\
\hline \multicolumn{3}{|l|}{ Protein Group } & & i|3228372 & 87808.8 & & & & & & & & & & & & \\
\hline adherin-15 [Rattus nor & & & & j|46485501 & 88045.9 & & & & & & & & & & & & \\
\hline
\end{tabular}

Peptide Information

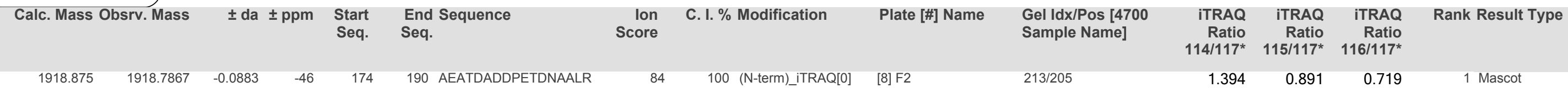

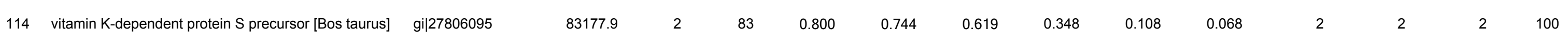
Peptide Information

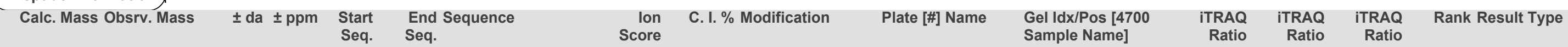

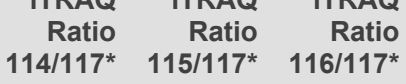




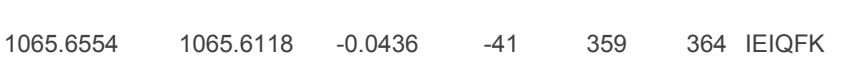

$\begin{array}{llllll}1683.8336 & 1683.7052 & -0.1284 & -76 & 591 & 601 \\ \text { KDSFHSEDFQR }\end{array}$
$40 \quad 96.924$ (N-term) iTRAQ[0], [5] F5 and F10 329/321

$\left.44 \quad 98.75 \begin{array}{l}\text { (N)-term) iTRAQ[0]] } \\ \text { Lysine(K)_iTRAQ[1] }\end{array}\right]$ [6] F12 and F9 and F7 211/203 $\begin{array}{llll}0.558 & 0.650 & 0.558 & 1 \text { Mascot }\end{array}$

$\begin{array}{llll}1.148 & 0.852 & 0.686 & 1 \text { Mascot }\end{array}$

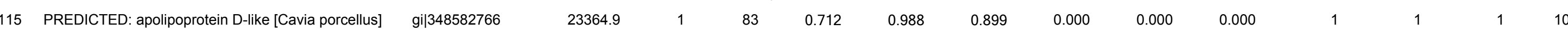

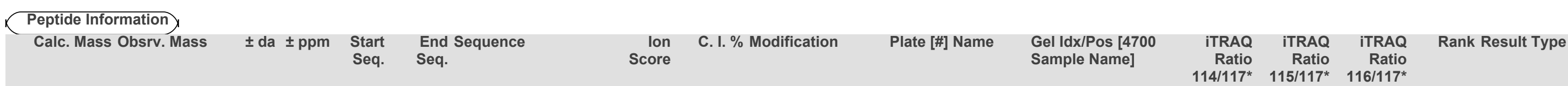

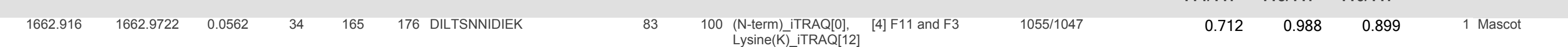

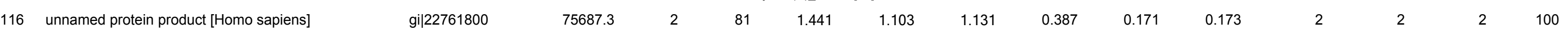

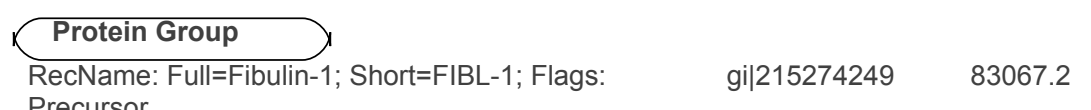

(1)

fibulin-1 isoform D precursor [Homo sapiens] $\quad$ gil34734066 $\quad 83095.2$

fibulin-1 isoform D precursor [Homo sapiens] $\quad$ gil13661193 $\quad 83128.3$

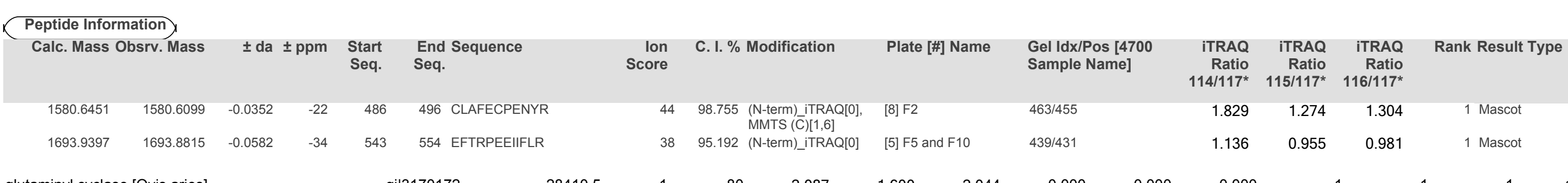

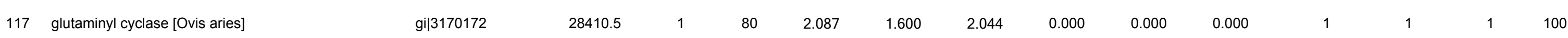

Peptide Information

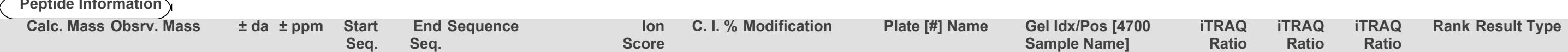

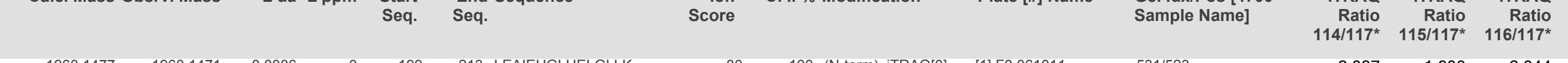

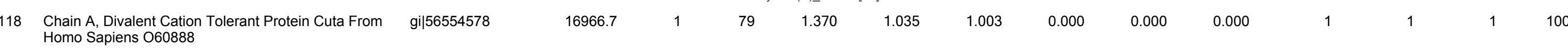
Protein Group
brain acetylcholinesterase putative membrane anchor
[Homo sapiens] protein CutA isoform 2 [Homo sapiens] $\quad$ gil $\quad 17706244 \quad 17922.5$

\begin{tabular}{|c|c|c|c|c|c|c|c|c|c|c|c|}
\hline Calc. Mass Obsrv. Mass & \pm da $\pm \mathrm{ppm}$ & $\begin{array}{l}\text { Start } \\
\text { Seq. }\end{array}$ & $\begin{array}{l}\text { End Sequence } \\
\text { Seq. }\end{array}$ & $\begin{array}{l}\text { Ion } \\
\text { Score }\end{array}$ & C. I. \% Modification & Plate [\#] Name & $\begin{array}{l}\text { Gel Idx/Pos [4700 } \\
\text { Sample Name] }\end{array}$ & $\begin{array}{r}\text { iTRAQ } \\
\text { Ratio } \\
114 / 117^{*}\end{array}$ & $\begin{array}{r}\text { iTRAQ } \\
\text { Ratio } \\
115 / 117^{*}\end{array}$ & $\begin{array}{r}\text { iTRAQ } \\
\text { Ratio } \\
116 / 117^{*}\end{array}$ & Rank Result Type \\
\hline 1677.9296 & 0.0585 & 93 & 106 TQSSLVPALTDFVR & 79 & 100 (N-term)_iTRAQ[0] & {$[8] \mathrm{F} 2$} & $474 / 466$ & 1.370 & 1.035 & 1.003 & 1 Mascot \\
\hline
\end{tabular}

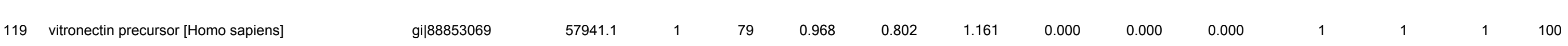
Protein Group $\quad$ gil13477169 57971.1

unnamed protein product [Homo sapiens] $\quad$ gil36573 $\quad 57978$

unnamed protein product [Homo sapiens] $\quad$ gil36575 $\quad 58037.1$

vitronectin [Pongo abelii] $\quad$ gi|197100902 $\quad 57802.9$

Peptide Information

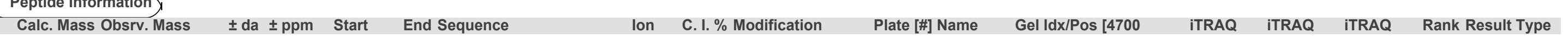




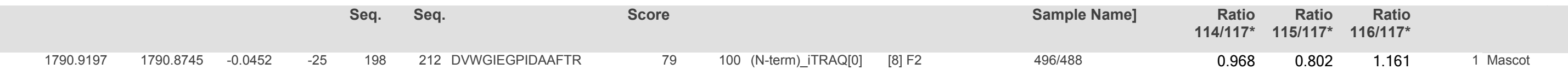

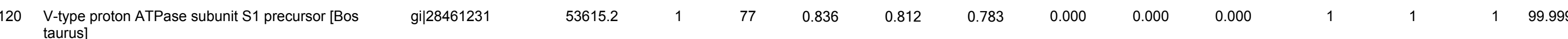
Peptide Information

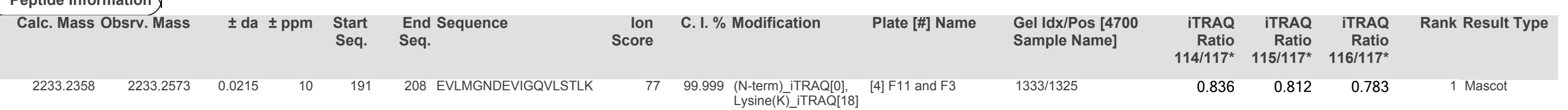

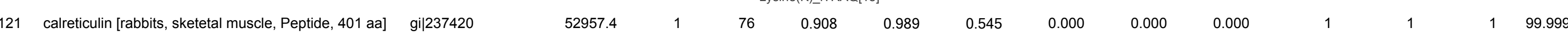
Peptide Information

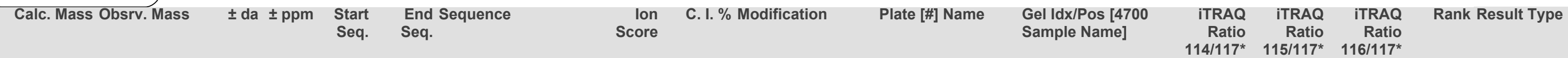

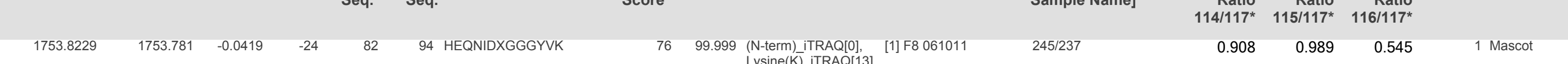

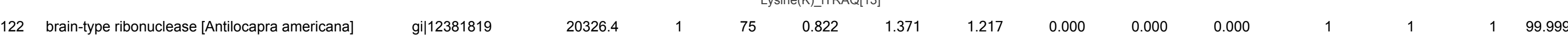

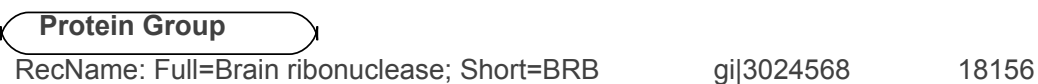

Peptide Information

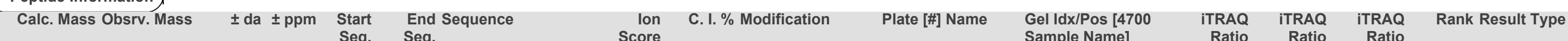

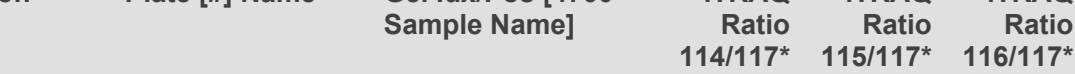

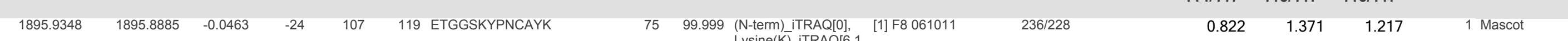

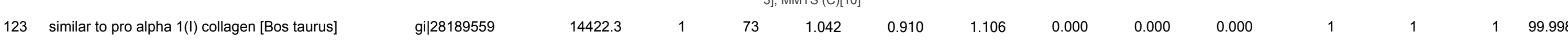
Protein Group

Collagen, type I, alpha 1 [Homo sapiens]

gi|22328092 148111.3

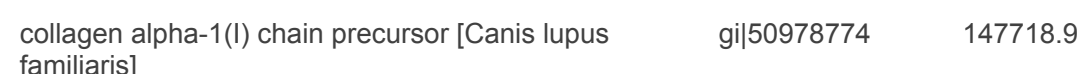

pro alpha $1($ I) collagen [Bos taurus] $\quad$ gi|14043011 34495.9

Peptide Information

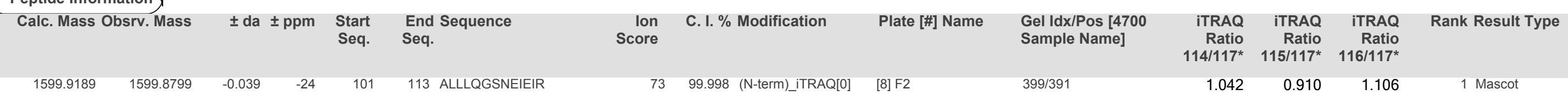

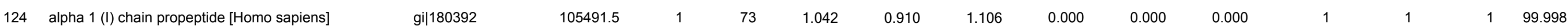

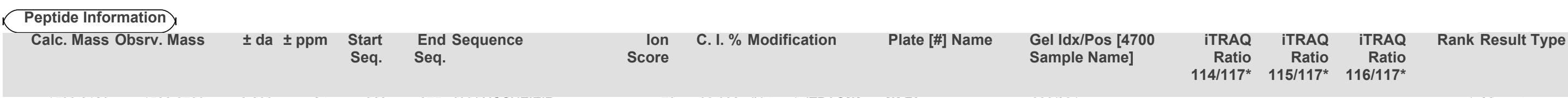

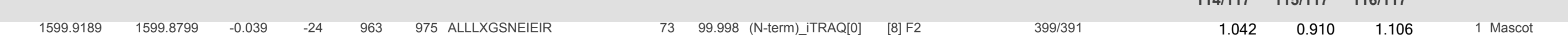

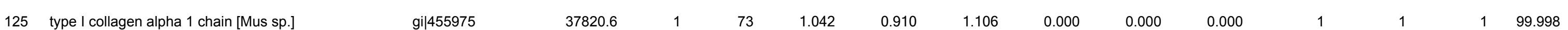

Protein Group

Col1a1 protein [Mus musculus]

gi|13096810 62883.2

$\begin{array}{lll}\text { Col1a1 protein [Mus musculus] } & \text { gil37589303 } & 125532.7\end{array}$

alpha-1 type I procollagen [Mus musculus]

gi|424104 38170,7 


\begin{tabular}{|c|c|c|c|c|c|c|c|c|c|c|c|}
\hline Calc. Mass Obsrv. Mass & \pm da \pm ppm & $\begin{array}{l}\text { Start } \\
\text { Seq. }\end{array}$ & $\begin{array}{l}\text { End Sequence } \\
\text { Seq. }\end{array}$ & $\begin{array}{l}\text { Ion } \\
\text { Score }\end{array}$ & C. I. \% Modification & Plate [\#] Name & $\begin{array}{l}\text { Gel Idx/Pos [4700 } \\
\text { Sample Name] }\end{array}$ & $\begin{array}{r}\text { iTRAQ } \\
\text { Ratio } \\
114 / 1117^{*}\end{array}$ & $\begin{array}{r}\text { iTRAQ } \\
\text { Ratio } \\
115 / 117^{*}\end{array}$ & $\begin{array}{r}\text { iTRAQ } \\
\text { Ratio } \\
116 / 117^{*}\end{array}$ & Rank Result Type \\
\hline 1599.8799 & -0.039 & 246 & 258 ALLLQGSNEIELR & 73 & 99.998 (N-term)_iTRAQ[0] & ${ }^{[8] \mathrm{F} 2}$ & 399/391 & 1.042 & 0.910 & 1.106 & hascot \\
\hline
\end{tabular}

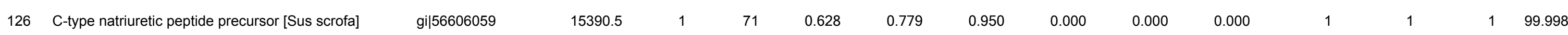
C-type natriuretic peptide protein

\begin{tabular}{|c|c|c|c|c|c|c|c|c|c|c|c|}
\hline Calc. Mass Obsrv. Mass & $\pm d a \pm p p m$ & $\begin{array}{l}\text { Start } \\
\text { Seq. }\end{array}$ & $\begin{array}{l}\text { End Sequence } \\
\text { Seq. }\end{array}$ & $\begin{array}{l}\text { Ion } \\
\text { Score }\end{array}$ & C. I. \% Modification & Plate [\#] Name & $\begin{array}{l}\text { Gel IdX/Pos [4700 } \\
\text { Sample Name] }\end{array}$ & $\begin{array}{r}\text { iTRAQ } \\
\text { Ratio } \\
114 / 117^{*}\end{array}$ & $\begin{array}{r}\text { iTRAQ } \\
\text { Ratio } \\
115 / 117^{*}\end{array}$ & $\begin{array}{r}\text { iTRAQ } \\
\text { Ratio } \\
116 / 117^{*}\end{array}$ & Rank Result Type \\
\hline 2011.0342 & 0.097 & 34 & $51 \underset{\mathrm{K}}{\mathrm{TP} P \mathrm{~T} E E V A E P Q A A G G G Q}$ & 71 & 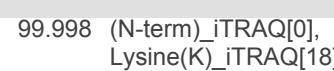 & [4] F11 and F3 & $947 / 1939$ & 0.628 & 0.779 & 0.950 & 1 Mascot \\
\hline
\end{tabular}

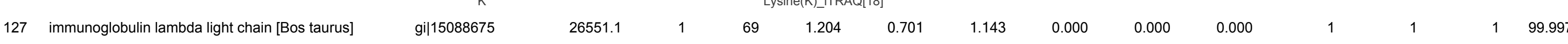
Protein Group

\begin{tabular}{|c|c|c|c|c|c|c|c|c|c|c|c|}
\hline Calc. Mass Obsrv. Mass & \pm da $\pm \mathrm{ppm}$ & $\begin{array}{l}\text { Start } \\
\text { Seq. }\end{array}$ & $\begin{array}{l}\text { End Sequence } \\
\text { Seq. }\end{array}$ & $\begin{array}{l}\text { Ion } \\
\text { Score }\end{array}$ & C. I. \% Modification & Plate [\#] Name & $\begin{array}{l}\text { Gel Idx/Pos [4700 } \\
\text { Sample Name] }\end{array}$ & $\begin{array}{r}\text { iTRAQ } \\
\text { Ratio } \\
114 / 117^{*}\end{array}$ & $\begin{array}{r}\text { iTRAQ } \\
\text { Ratio } \\
115 / 117^{*}\end{array}$ & $\begin{array}{r}\text { iTRAQ } \\
\text { Ratio } \\
116 / 117^{*}\end{array}$ & Rank Result Type \\
\hline 2018.8159 & -0.1231 & 212 & 227 GSYSCEVTHEGSTVTK & 69 & $\begin{array}{c}99.997 \text { (N-term) iTRAQ[0], } \\
\text { Lysine(KK) iTRAQ[16 } \\
\text { MMTS (C)[5] }\end{array}$ & {$[8] \mathrm{F} 2$} & $308 / 300$ & 1.204 & 0.701 & 1.143 & 1 Mascot \\
\hline
\end{tabular}

$128 \begin{aligned} & \text { extracellular superoxide dismutase [Cu-Zn] precursor } \\ & \text { [Oryctolagus cuniculus] }\end{aligned}$ Protein Group
[Homo sapiens]

[Homo sapiens]
$\begin{aligned} & \text { extracellular-superoxide dismutase (EC 1.15.1.1) } \\ & \text { [Homo sapiens] }\end{aligned} \quad$ gil338284 $\quad 27097.2$

\begin{tabular}{|c|c|c|c|c|c|c|c|c|c|c|c|}
\hline Calc. Mass Obsrv. Mass & \pm da $\pm p p m$ & $\begin{array}{l}\text { Start } \\
\text { Seq. }\end{array}$ & $\begin{array}{l}\text { End Sequence } \\
\text { Seq. }\end{array}$ & $\begin{array}{l}\text { Ion } \\
\text { Score }\end{array}$ & C. I. \% Modification & Plate [\#] Name & $\begin{array}{l}\text { Gel Idx/Pos [4700 } \\
\text { Sample Name] }\end{array}$ & $\begin{array}{r}\text { iTRAQ } \\
\text { Ratio } \\
114 / 117^{*}\end{array}$ & $\begin{array}{r}\text { iTRAQ } \\
\text { Ratio } \\
115 / 117^{*}\end{array}$ & $\begin{array}{r}\text { iTRAQ } \\
\text { Ratio } \\
116 / 117^{*}\end{array}$ & Rank Result Type \\
\hline 1481.8273 & 0.0441 & 181 & 193 AVVVHAGEDDLGR & 68 & 99.995 (N-term)_iTRAQ[0] & [3] F14,15,16 and F6 & $760 / 752$ & 0.542 & 0.846 & 0.718 & 1 Mascot \\
\hline
\end{tabular}

129 thyroxine-binding globulin precursor [Ovis aries] $\quad \begin{array}{llllllllllll} & \text { gi|155369640 } & 50563.6 & 1 & 67 & 0.655 & 0.622 & 1.069 & 0.000 & 0.000 & 0.000 & 1\end{array}$

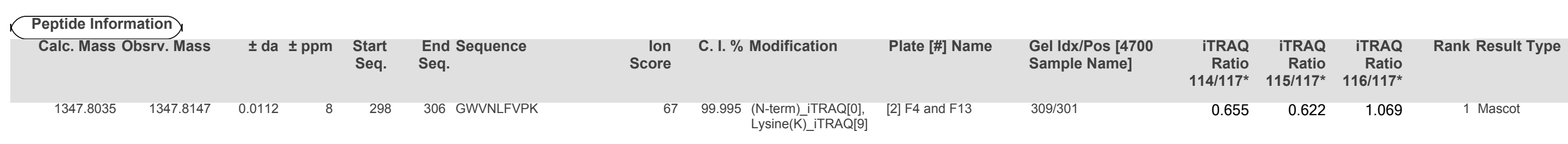

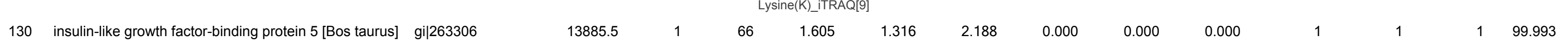
Protein Group

IGFBP5 [Homo sapiens] $\quad$ gil48145735 34534

insulin-like growth factor binding protein 5 [Mus $\quad$ gil437125
musculus]

insulin-like growth factor binding protein-5 [Equus $\quad$ gi|56849566 12886 
insulin-like growth factor-binding protein $5 \quad$ gil1586428 $\quad 34346.8$

insulin-like growth factor-binding protein 5 precursor $\quad$ gil10834982 34548

$\begin{array}{lll}\text { insulin-like growth factor-binding protein } 5 \text { precursor } & \text { gil70909322 } 34349.8 \\ \text { [Mus musculus] }\end{array}$

[Mus musculus]

insulin-like growth factor-binding protein 5 precursor $\quad$ gil6981088 34321.7
[Rattus norvegicus]

insulin-like growth factor-binding protein 5 precursor $\quad$ gi|47523008 $\quad 34360.8$
[Sus scrofa]

Peptide Information

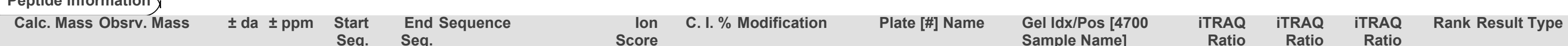

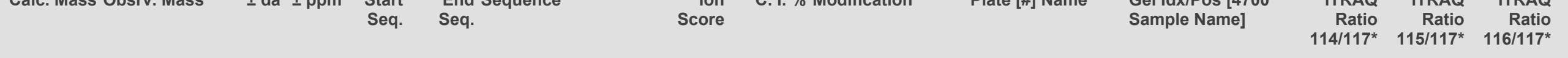

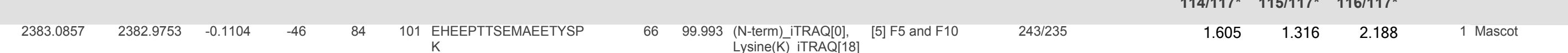

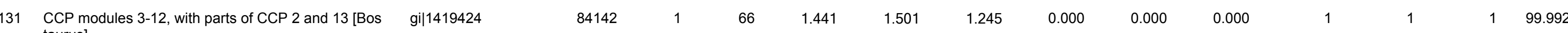
Peptide Information

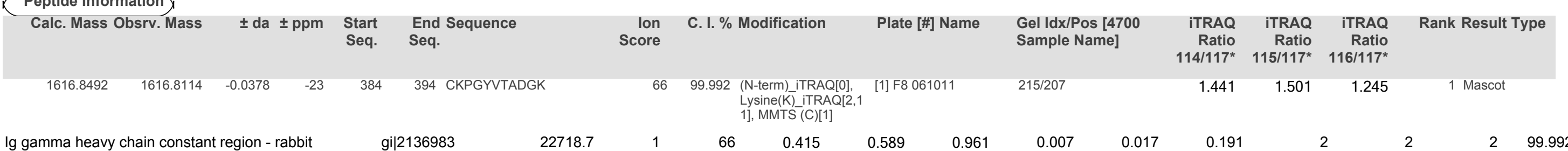

132 Ig gamma heavy chain constant region - rabbit gil2136983
(fragment)

\begin{tabular}{lll} 
Protein Group & \\
\hline Ig gamma H-chain C-region [Oryctolagus cuniculus] & gil457366
\end{tabular}

Ig gamma H-chain [Oryctolagus cuniculus] $\quad$ gil165128 $\quad 48079.4$

RecName: Full=Ig gamma chain C region $\quad$ gi|121088 $\quad 38867.8$

\section{Peptide Information}

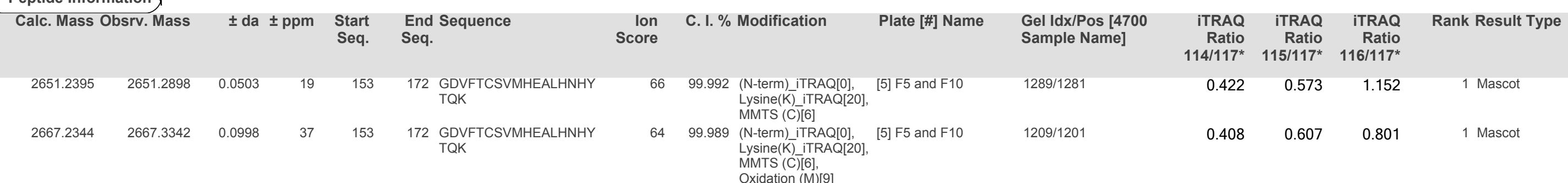

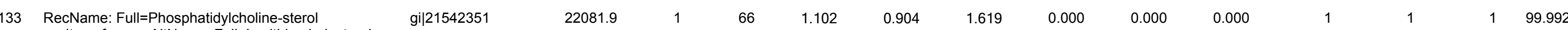
acyltr

\section{Peptide Information}

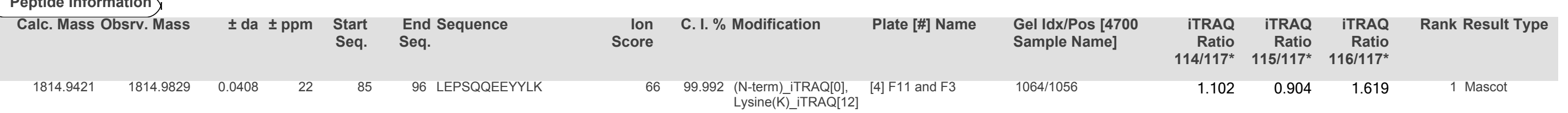

134 RecName: Full=Plasminogen

gi|51702791

15564.2

$\begin{array}{lll}65 & 0.835 & 0.990\end{array}$

$0.000 \quad 0.000 \quad 0.000$

Peptide Information

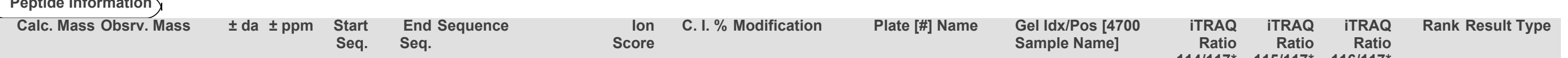

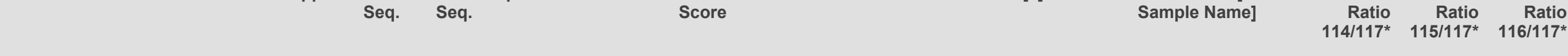

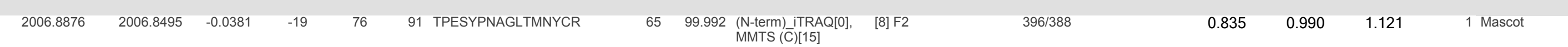




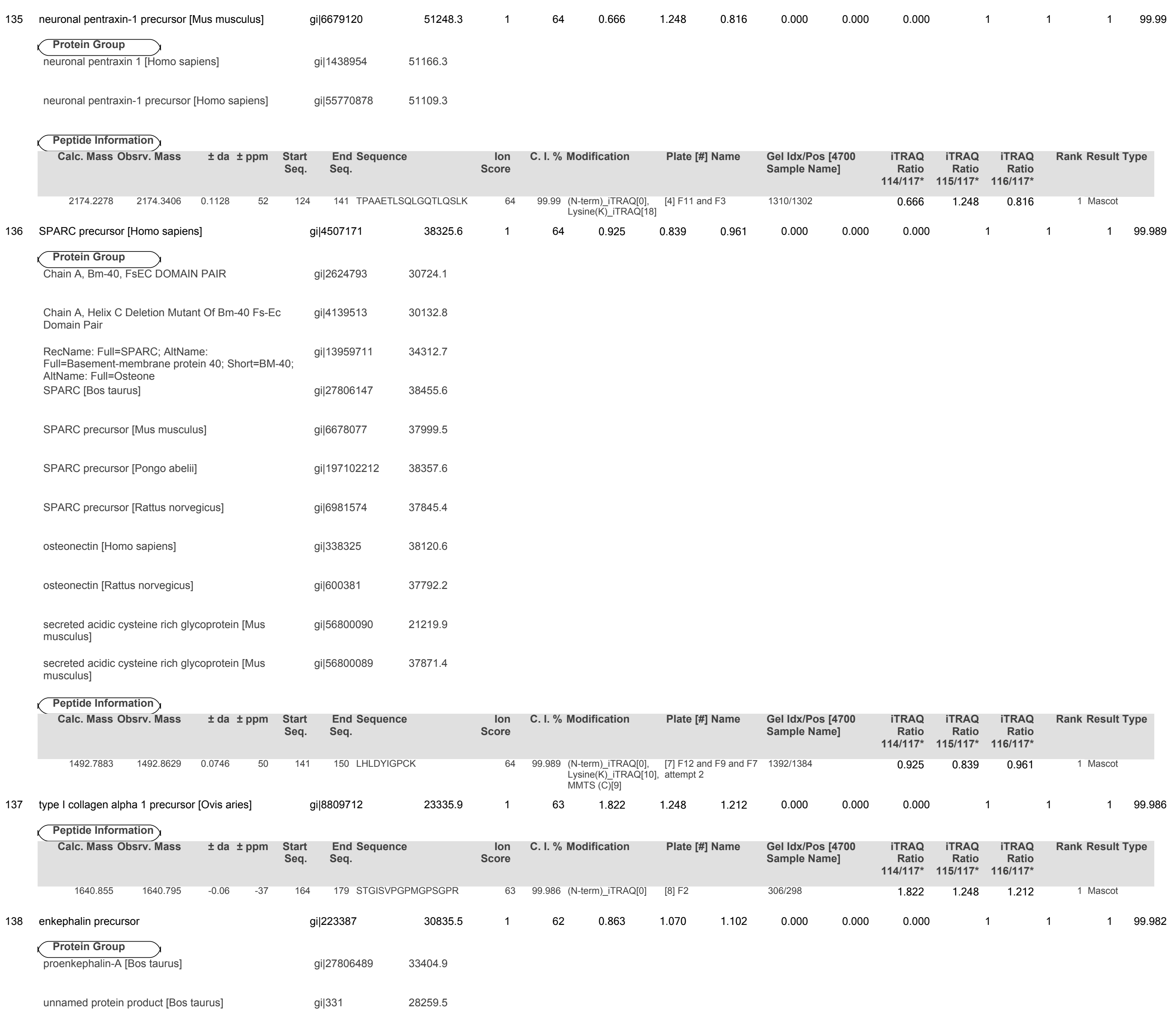


Peptide Information

\begin{tabular}{|c|c|c|c|c|c|c|c|c|c|c|c|}
\hline Calc. Mass Obsrv. Mass & \pm da \pm ppm & $\begin{array}{l}\text { Start } \\
\text { Seq. }\end{array}$ & $\begin{array}{l}\text { End Sequence } \\
\text { Seq. }\end{array}$ & $\begin{array}{l}\text { lon } \\
\text { Score }\end{array}$ & C. I. \% Modification & Plate [\#] Name & $\begin{array}{l}\text { Gel IdxlPos [4700 } \\
\text { Sample Name] }\end{array}$ & $\begin{array}{r}\text { iTAQ } \\
\text { Ratio } \\
114 / 117^{*}\end{array}$ & $\begin{array}{r}\text { iRAQ } \\
\text { Ratio } \\
115 / 117^{*}\end{array}$ & $\begin{array}{r}\text { iRAQ } \\
\text { Ratio } \\
116 / 117^{*}\end{array}$ & Rank Result Type \\
\hline
\end{tabular}

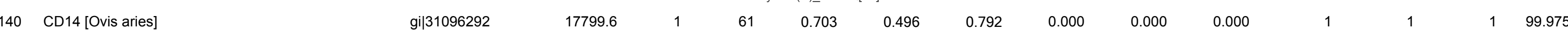

Peptide Information

\begin{tabular}{|c|c|c|c|c|c|c|c|c|c|c|c|}
\hline Calc. Mass Obsrv. Mass & \pm da $\pm \mathrm{ppm}$ & $\begin{array}{l}\text { Start } \\
\text { Seq. }\end{array}$ & $\begin{array}{l}\text { End Sequence } \\
\text { Seq. }\end{array}$ & $\begin{array}{l}\text { lon } \\
\text { Score }\end{array}$ & C. I. \% Modification & Plate [\#] Name & $\begin{array}{l}\text { Gel Id dX/Pos [4700 } \\
\text { Sample Name] }\end{array}$ & $\begin{array}{r}\text { iTRAQ } \\
\text { Ratio } \\
114 / 117^{*}\end{array}$ & $\begin{array}{r}\text { iTRAQ } \\
\text { Ratio } \\
115 / 117^{*}\end{array}$ & $\begin{array}{r}\text { iTRAQ } \\
\text { Ratio } \\
116 / 117^{*}\end{array}$ & Rank Result Type \\
\hline
\end{tabular}

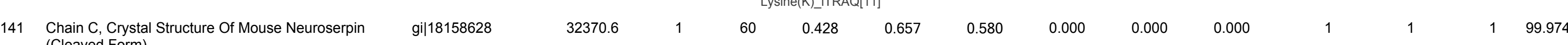

Protein Group

Serine (or cysteine) peptidase inhibitor, clade I, member gi|13904990 49462.2

402.2

neuroserpin precursor [Mus musculus] $\quad$ gil6678091 49534.2

\section{Peptide Information}

\begin{tabular}{|c|c|c|c|c|c|c|c|c|c|c|c|}
\hline Calc. Mass Obsrv. Mass & \pm da \pm ppm & $\begin{array}{l}\text { Start } \\
\text { Seq. }\end{array}$ & $\begin{array}{l}\text { End Sequence } \\
\text { Seq. }\end{array}$ & $\begin{array}{l}\text { lon } \\
\text { Score }\end{array}$ & C. I. \% Modification & Plate [\#] Name & $\begin{array}{l}\text { Gel Idx/Pos [4700 } \\
\text { Sample Name] }\end{array}$ & $\begin{array}{r}\text { iTRAQ } \\
\text { Ratio } \\
114 / 117^{*}\end{array}$ & $\begin{array}{r}\text { iTRAQ } \\
\text { Ratio } \\
115 / 117^{*}\end{array}$ & $\begin{array}{r}\text { iTRAQ } \\
\text { Ratio } \\
116 / 117^{*}\end{array}$ & Rank Result Type \\
\hline 1675.9265 & 0.0403 & 173 & 184 AQLIEEWANSVK & 60 & 99.974 (N-term) _iTRAQ & F4 and F13 & 2900/282 & 0.428 & 0.657 & 0.580 & lascot \\
\hline
\end{tabular}

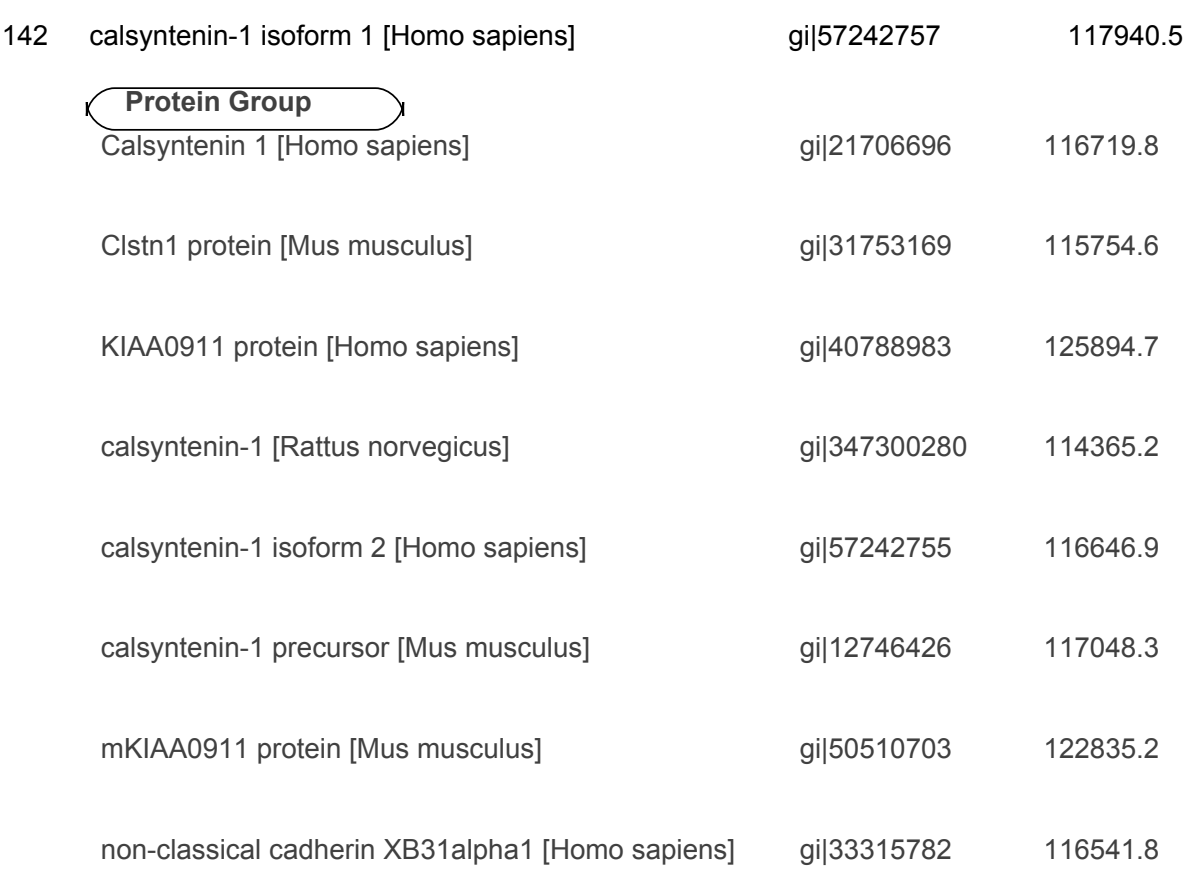




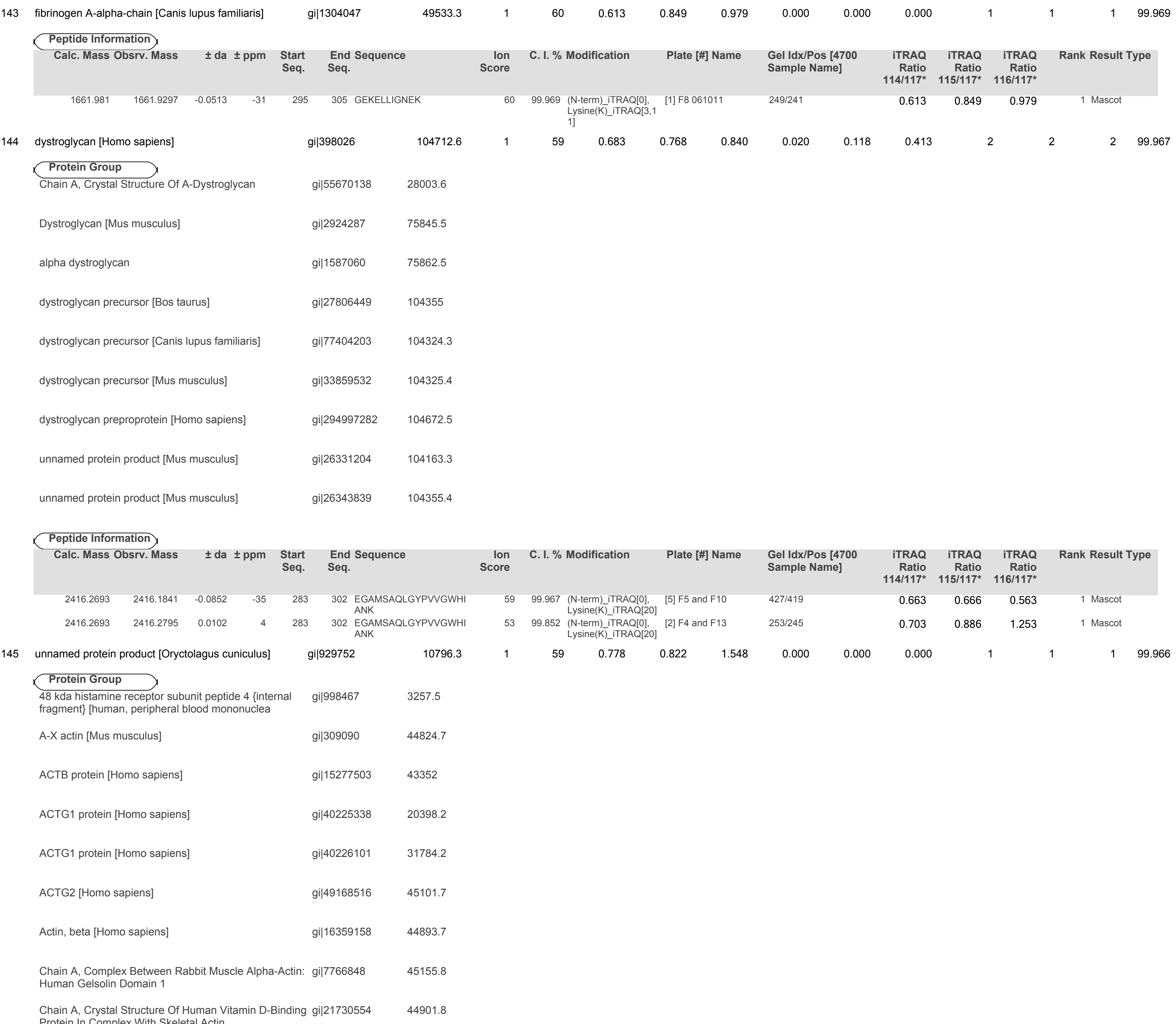

Chain A, Crystal Structure Of A-Dystroglycan

Dystroglycan [Mus musculus]

alpha dystroglycan

dystroglycan precursor [Bos taurus]

dystroglycan precursor [Canis lupus familiaris]

dystroglycan precursor [Mus musculus]

dystroglycan preproprotein [Homo sapiens]

unnamed protein product [Mus musculus]

unnamed protein product [Mus musculus]

gi|55670138 28003

gi|2924287 $\quad 75845.5$

gil1587060 $\quad 75862.5$

gi|27806449 104355

gi|77404203 $\quad 104324.3$

gi|33859532 $\quad 104325.4$

gi|294997282 104672.5

gi|26331204 $\quad 104163.3$

gi|26343839 104355.4

\section{Peptide Information}

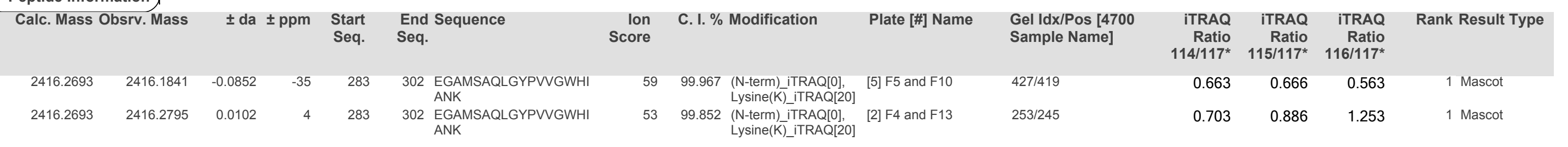

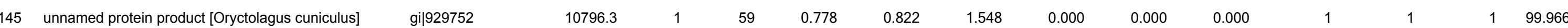

fragments [hom

A-X actin [Mus musculus]

gi|309090 44824.7

ACTB protein [Homo sapiens]

gi|15277503 43352

ACTG1 protein [Homo sapiens]

gil40225338 20398.2

ACTG1 protein [Homo sapiens]

gil40226101 31784.2

ACTG2 [Homo sapiens]

gi|49168516 45101.

Actin, beta [Homo sapiens]

gi|16359158 $\quad 44893.7$

Chain A, Complex Between Rabbit Muscle Alpha-Actin: gi|7766848 45155

Gelsolin Domain 1

Chain A, Crystal Structure Of Human Vitamin D-Binding gi|21730554

44901.8 
PREDICTED: actin, alpha skeletal muscle-like isoform 3 gi|297281875

[Macaca mulatta]

RecName: Full=Actin, cytoplasmic 1; AltName: $\quad$ gil|47116231
Full=Beta-actin; Contains: RecName: Full=Actin, cytopl

RecName: Full=Actin, cytoplasmic 1; AltName:
Full=Beta-actin; Contains: RecName: Full=Actin, cytopl

$\begin{array}{ll}\text { RecName: Full=Putative beta-actin-like protein 3; } & \text { gi|74739412 } \\ \text { AltName: Full=Kappa-actin; AltName: Full=POTE ank } & \end{array}$

Unknown (protein for IMAGE:3538275) [Homo sapiens] gil16924319

Unknown (protein for IMAGE:3897065) [Homo sapiens] gil16306948

actin prepeptide [Homo sapiens]

gil178067 $\quad 39508.8$

actin, alpha 1, skeletal muscle [Homo sapiens]

gi|56204817 $\quad 34897.6$

actin, alpha cardiac muscle 1 proprotein [Homo

gil4885049 $\quad 45149.8$

actin, alpha skeletal muscle [Bos taurus]

gil27819614 $\quad 45266.9$

actin, alpha skeletal muscle [Homo sapiens]

gi|4501881 $\quad 45181.8$

actin, aortic smooth muscle [Homo sapiens]

gi|4501883 $\quad 45185.8$

actin, beta [Homo sapiens]

gi|14250401 $\quad 44136.4$

actin, beta [Macaca fascicularis]

gi|57281683 14491.4

actin, cytoplasmic 1 [Cricetulus griseus]

gi|347360906 $\quad 44868.7$

actin, cytoplasmic 1 [Homo sapiens]

gi|4501885 $\quad 44867.7$

actin, cytoplasmic 1 [Oryctolagus cuniculus]

gi|156119364 44886.7

actin, cytoplasmic 1 [Pongo abelii]

gi|197099682 $\quad 44866.7$

actin, cytoplasmic 2 [Homo sapiens]

gi|4501887 $\quad 44923.8$

actin, gamma-enteric smooth muscle isoform 1
precursor [Homo sapiens]

gi|4501889

45053.7

alpha 2 actin [Bos taurus]

gi|61553131 $\quad 48919.9$

alpha-actin (AA 27-375) [Mus musculus]

gi|49870

42147.7

alpha-actin (aa 40-375) [Mus musculus]

gi|49864

40709.8 


\begin{tabular}{|c|c|c|}
\hline alpha-actin [Homo sapiens] & gi|178027 & 45284.8 \\
\hline alpha-cardiac actin [Mus musculus] & gi|387090 & 44869.8 \\
\hline alpha-smooth muscle actin - rabbit (fragment) & gi|2136927 & 27633.2 \\
\hline beta actin [Cricetinae gen. sp.] & $g i \mid 2318133$ & 44859.8 \\
\hline beta actin [Cricetinae gen. sp.] & gi|2318135 & 34828.6 \\
\hline beta actin [Lama glama] & gi|29603621 & 36251.3 \\
\hline beta actin [Meriones unguiculatus] & gi|41387701 & 22995.6 \\
\hline beta actin [Sus scrofa] & gi $\mid 476332$ & 27871.1 \\
\hline beta-actin FE-3 [Rattus norvegicus] & gi|13516471 & 16264.2 \\
\hline beta-actin [Didelphis virginiana] & gi|61375223 & 36150.5 \\
\hline beta-actin [Felis catus] & gi|11191964 & 34425.7 \\
\hline beta-actin [Macaca fuscata] & gi|6636344 & 31385.2 \\
\hline beta-actin [Macaca mulatta] & gi|40744574 & 33666.2 \\
\hline beta-actin [Marmota monax] & gi|9864780 & 34175.4 \\
\hline beta-actin [Mus musculus] & gi|48237757 & 9302.5 \\
\hline beta-actin [Mustela putorius furo] & gi|2724046 & 38781.9 \\
\hline beta-actin [Oryctolagus cuniculus] & gi|12240086 & 16512.3 \\
\hline beta-actin [Ovis aries] & gi|8809716 & 26728.3 \\
\hline beta-actin [Ovis aries] & gi|2665740 & 29781.1 \\
\hline beta-actin [Sus scrofa] & gi|20068082 & 19962.9 \\
\hline beta-actin-like [Canis lupus familiaris] & gi|55741585 & 44749.9 \\
\hline beta-actin-like protein 2 [Mus musculus] & gi|30425250 & 44990.9 \\
\hline cardiac actin [Canis lupus familiaris] & gi|11493749 & 8151 \\
\hline cardiac alpha actin [Equus caballus] & gil14456493 & 8899.3 \\
\hline cytoskeletal beta actin [Sus scrofa] & gi|45269029 & 47967.2 \\
\hline gamma actin-like protein [Mus musculus] & gi|6425087 & 46678.3 \\
\hline
\end{tabular}




$\begin{array}{llc}\text { gamma-actin [Homo sapiens] } & \text { gi|178045 } & 28109.3 \\ \text { gamma-actin [Mus musculus] } & \text { gi|809561 } & 44150.4 \\ \text { mutant beta-actin (beta'-actin) [Homo sapiens] } & \text { gi|28336 } & 44943.6 \\ \text { put. beta-actin (aa 27-375) [Mus musculus] } & \text { gi|498668 } & 42128.5 \\ \text { similar to beta actin [Bos taurus] } & \text { gi|28189611 } & 22161 \\ \text { skeletal muscle alpha-actin [Mus musculus] } & \text { gi|387082 } & 40672.6 \\ \text { smooth muscle alpha actin [Mesocricetus auratus] } & \text { gi|21070328 } & 17433.8 \\ \text { smooth muscle gamma-actin [Mus musculus] } & \text { gi|950002 } & 45623.9 \\ \text { unnamed protein product [Mus musculus] } & \text { gi|12852068 } & 45077.8\end{array}$

\section{Peptide Information}

\begin{tabular}{|c|c|c|c|c|c|c|c|c|c|c|c|}
\hline ass Obsrv. Mass & \pm da \pm ppm & $\begin{array}{l}\text { Start } \\
\text { Seq. }\end{array}$ & $\begin{array}{l}\text { End Sequence } \\
\text { Seq. }\end{array}$ & $\begin{array}{l}\text { lon } \\
\text { Score }\end{array}$ & C. I. \% Modification & Plate [\#] Name & $\begin{array}{l}\text { Gell Idx/Pos [4700 } \\
\text { Sample Name] }\end{array}$ & $\begin{array}{r}\text { iTRAQ } \\
\text { Ratio } \\
114 / 117^{*}\end{array}$ & $\begin{array}{r}\text { iTRAQ } \\
\text { Ratio } \\
115 / 117^{*}\end{array}$ & $\begin{array}{r}\text { iTRAQ } \\
\text { Ratio } \\
116 / 117^{*}\end{array}$ & Rank Result Type \\
\hline $0210111^{\circ}$ & -0.0532 & 48 & 63 SYELPDGQVITIGNER & 59 & 99.966 (N-term)_iTRAQLIC & [8] F2 & 421/413 & 0.778 & 0.822 & 1.548 & 1 Mascot \\
\hline
\end{tabular}

146 PREDICTED: beta-actin-like protein 2-like [Canis lupus
famililiaris]

\begin{tabular}{|c|c|c|c|c|c|c|c|c|c|c|c|}
\hline \multicolumn{12}{|l|}{ Peptide Information } \\
\hline Calc. Mass Obsrv. Mass & \pm da $\pm \mathrm{ppm}$ & $\begin{array}{l}\text { Start } \\
\text { Seq. }\end{array}$ & $\begin{array}{l}\text { End Sequence } \\
\text { Seq. }\end{array}$ & $\begin{array}{l}\text { lon } \\
\text { score }\end{array}$ & C. I. \% Modification & Plate [\#] Name & $\begin{array}{l}\text { Gel Idx/Pos [4700 } \\
\text { Sample Name] }\end{array}$ & $\begin{array}{r}\text { iTRAQ } \\
\text { Ratio } \\
114 / 117^{*}\end{array}$ & $\begin{array}{r}\text { iTRAQ } \\
\text { Ratio } \\
115 / 117^{\star}\end{array}$ & $\begin{array}{r}\text { iTRAQ } \\
\text { Ratio } \\
116 / 117^{\star}\end{array}$ & Rank Result Type \\
\hline 1934.9944 & -0.0532 & 240 & 255 SYELPDGQVITLGNER & 59 & 99.966 (N-term)_iTRAQ[0] & {$[8] \mathrm{F} 2$} & $421 / 413$ & 0.778 & 0.822 & 1.548 & Mascot \\
\hline
\end{tabular}
147 truncated tenascin XB [Homo sapiens]
gil6448761 $\quad 31411.2$
159
$1.296 \quad 1.895$
$1.022 \quad 0.000$
0.000
0.000
$1 \quad 1$
199.964

Protein Group

tenascin-X [Bos taurus]

gi|74706767 34917.8

Peptide Information

gi|27807269 $\quad 469186.8$

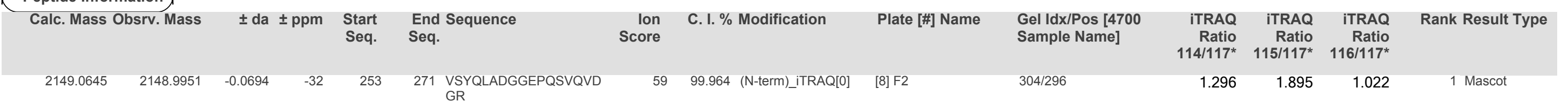
29430.1

$\begin{array}{llll}1 & 57 & 1.124 & 0.810\end{array}$

$0.589 \quad 0.564$

$0.110 \quad 0.255$

22

$2 \quad 99.945$

Protein Group

V-set and transmembrane domain containing 2A [Mus gi|20072584
musculus]

28028.4

V-set and transmembrane domain containing 2A [Mus gi|56205459

V-set and transmembrane domain containing 2A [Mus gil56205460
musculus]

musculus]

V-set and transmembrane domain-containing protein 2A gi|281182810
precursor [Mus musculus]

hypothetical protein MGC33530 [Homo sapiens]

gi|51094556

33336.9

27929.4

28159.5

29286.9 
Peptide Information

\begin{tabular}{|c|c|c|c|c|c|c|c|c|c|c|c|c|}
\hline Calc. Mass Obsrv. Mass & \pm da & ppm & $\begin{array}{l}\text { Start } \\
\text { Seq. }\end{array}$ & $\begin{array}{l}\text { End Sequence } \\
\text { Seq. }\end{array}$ & $\begin{array}{l}\text { lon } \\
\text { Score }\end{array}$ & C. I. \% Modification & Plate [\#] Name & $\begin{array}{l}\text { Gel Id dx/Pos [4700 } \\
\text { Sample Name] }\end{array}$ & $\begin{array}{r}\text { iTRAQ } \\
\text { Ratio } \\
114 / 117^{*}\end{array}$ & $\begin{array}{r}\text { iTRAQ } \\
\text { Ratio } \\
115 / 117^{*}\end{array}$ & $\begin{array}{r}\text { iTRAQ } \\
\text { Ratio } \\
116 / 117^{*}\end{array}$ & Rank Result Type \\
\hline 1791.9662 & 0.054 & 30 & 128 & 140 VTDANYGELQEHK & 57 & 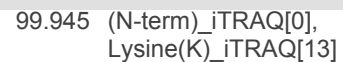 & {$[3] F 14,15,16$ and $F 6$} & $767 / 759$ & 1.688 & 0.920 & 0.411 & 1 Mascot \\
\hline 1791.9122 & 0.0594 & 33 & 128 & 140 VTDANYGELQEHK & 42 & 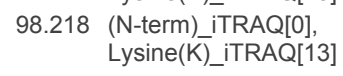 & $\begin{array}{l}{[7] F 12 \text { and } F 9 \text { and } F 7} \\
\text { attempt } 2\end{array}$ & $1246 / 1238$ & 0.749 & 0.713 & 0.844 & 1 Mascot \\
\hline
\end{tabular}

149 GOLPH2 [Homo sapiens]

golgi membrane protein 1 [Homo sapiens]

\section{Peptide Information}

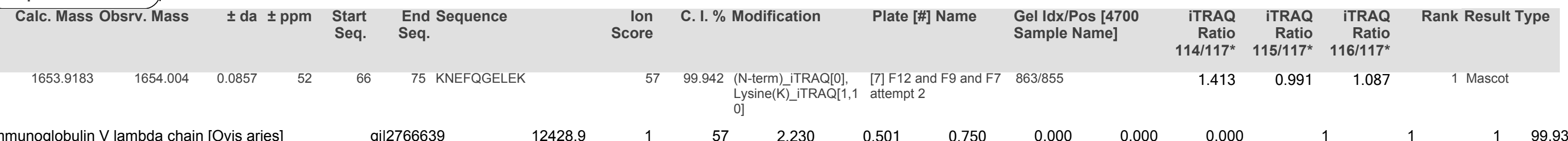

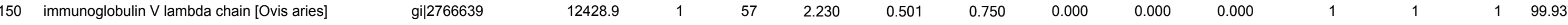
Peptide Information

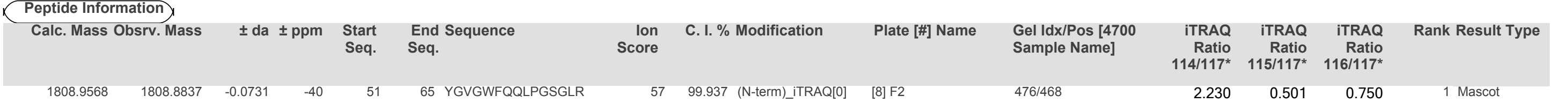

151 immunoglobulin lambda light chain VLJ region [Homo gi|21669651 30883.4

\section{Protein Group}

AG=monoclonal IgM lambda VIII gil632814 light chain $\{$ region\} hhum

Chain A, Bence Jones Protein Cle, A Lambda lii $\quad$ gi|2194054

13347.6

Immunoglobulin Light- Chain Dimer

24521.

Chain A, Crystal Structure Of Monoclonal Antibody gil40889110 24644.1

Chain A, Crystal Structure Of The Fab Fragment From gil443559 The Human Myeloma Immunoglobulin Igg Hil At 1.8

Chain A, Three-Dimensional Structure of An gi|21465749 Immunoglobulin Light Chain Dimer Acting As A Letha

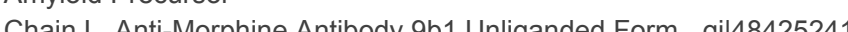

Chain L, Cocaine Hydrolytic Antibody $15 a 10 \quad$ gil46014931 24571.2

Chain L, Crystal Structure Of A Human Igm Rheumatoid gil3659942
Factor Fab In Complex With Its Autoantigen lgg Fc

Chain L, Crystal Structure Of An Anti-Carbohydrate $\quad$ gi|9954938 24105

Complex With Antigen

Chain L, Crystal Structure Of An Anti-Carbohydrate $\quad$ gi|9954942 24119

Antibody Directed Against Vibrio Cholerae 01 In

Chain $L$, Crystal Structure Of Humen Factor $1 x$ G

gi|42543068 
Domain In Complex Of An Inhibitory Antibody, 10c12

Chain L, Fab Fragment

Chain L, Free Conformation Ab1 Of The Ige Spe-7

gi|42543147

13308.8

Chain L, High Resolution Structures Of Antibody Fab gil494368 24807.2 Fragment Complexed With Cell-Surface
Oligosaccharide Of Pathogenic Salmonella

Chain L, How The Anti-(Metal Chelate) Antibody

gi|443045

24838.2

-Ray Structures

Complexes With Different Metals In The Chelate

gi|1633154 24766.2

Chain L, Recognition Of A Cell-Surface gil494374

24790.1

Oligo-Saccharide Of Pathogenic Salmonella By An

Chain L, Refined Three-Dimensional Structure Of The gil515129

mbda Antibody

Chain L, The Three-Dimensional Structure of An
Anti-Sweetener Fab Nc10.14, Shows The Extent Of

Anti-Sweetener Fab, Nc10.14, Shows The Extent

Structural Diversity In Antigen Recognition By

Chain L, Three-Dimensional Structure Of A Human Fab gi|2914164
With High Affinity For Tetanus Toxoid

Chain W, Murine 6a6 Fab In Complex With Humanized gi|34810347

IGL@ protein [Homo sapiens]

gi|48735306 28028.1

IGL@ protein [Homo sapiens]

gi|49522736 $\quad 27880.8$

IGL@ protein [Homo sapiens

gi|21595392 27094.

IGL@ protein [Homo sapiens]

gi|20380868 $\quad 26782.3$

IGL@ protein [Homo sapiens]

gil49256434 26787.4

IGL@ protein [Homo sapiens]

gi|49258104 26655.2

IGL@ protein [Homo sapiens]

gi|47939570 26911.4

IGL@ protein [Homo sapiens]

gi|21410208 $\quad 27442$.

$\lg \mathrm{AL}$

gi|229536

25060.5

Ig L-chain V-region [Homo sapiens]

gil27552515 25023.6

$\lg \vee$ lambda

gi|226513 $\quad 15750.9$

Ig VJC-region [Homo sapiens]

gil306977

26885.4

Ig lambda C Sut

gi|224503

13263.8

Ig lambda VI THO

gi|224892

25411.5

Ig lambda chain (BJP-DIA) - human

gi|539617

24726.3 


\begin{tabular}{|c|c|c|}
\hline Ig lambda chain - human & gi|106637 & 26662.2 \\
\hline Ig lambda chain - human & gi|106642 & 26700.2 \\
\hline Ig lambda chain - human & gi|106643 & 26734.5 \\
\hline Ig lambda chain - human & gi|106640 & 26917.4 \\
\hline Ig lambda chain - human & gi|106639 & 26723.4 \\
\hline Ig lambda chain - human & gi|106655 & 27005.6 \\
\hline Ig lambda chain - human & gi|106653 & 26623.3 \\
\hline Ig lambda chain - human & gi|106651 & 26506.2 \\
\hline Ig lambda chain - human & gi|106649 & 28312.2 \\
\hline Ig lambda chain - human & gi|106648 & 26653.1 \\
\hline Ig lambda chain - human & gi|106636 & 26618.1 \\
\hline Ig lambda chain - human & gi|106645 & 26947.4 \\
\hline Ig lambda chain - human (fragment) & gi|106657 & 26903.5 \\
\hline Ig lambda chain - human (fragment) & gi|106660 & 26675.3 \\
\hline Ig lambda chain - human (fragment) & gi|106656 & 17109.4 \\
\hline Ig lambda chain - human (fragment) & gi|106661 & 22181.1 \\
\hline Ig lambda chain V region (Zim) - human (fragment) & gil|87899 & 12695.4 \\
\hline Ig lambda chain V region - human & gi|478602 & 24485.3 \\
\hline Ig lambda chain $V$ region - human & gi|346196 & 13069.5 \\
\hline Ig lambda chain V region - human & gi|7439053 & 24713.4 \\
\hline Ig lambda chain $V$ region - human (fragment) & gi|478653 & 16264.1 \\
\hline Ig lambda chain $V$ region MabB23 - mouse & gi|2135590 & 24658.2 \\
\hline Ig lambda chain V-region (V-J) [Homo sapiens] & gi|186112 & 15814.9 \\
\hline Ig lambda chain V-region [Homo sapiens] & gi|1864143 & 15653.1 \\
\hline Ig lambda chain precursor - human & gil 87890 & 26978.5 \\
\hline $\begin{array}{l}\text { Ig lambda chain precursor V region (JP-DL-3) - human } \\
\text { (fragment) }\end{array}$ & gi|l87891 & 15229.6 \\
\hline
\end{tabular}




\begin{tabular}{|c|c|c|}
\hline $\begin{array}{l}\text { Ig lambda light chain variable region (VJC) [Homo } \\
\text { sapiens] }\end{array}$ & gil439517 & 14408 \\
\hline Ig lambda,anti-Rh(c) & gi|227167 & 25272.6 \\
\hline $\begin{array}{l}\text { Ig lambda-1 chain V region (hybridoma Se155-4) - } \\
\text { mouse }\end{array}$ & gi|627941 & 12752.4 \\
\hline Ig rearranged L-chain V-region [Homo sapiens] & gi|28559048 & 17981.9 \\
\hline $\begin{array}{l}\text { IgG1/L immunoglobulin light chain VL2 variable region } \\
\text { [Homo sapiens] }\end{array}$ & gi|33340655 & 13207.4 \\
\hline IgM heavy chain variable region [Homo sapiens] & gil4100378 & 13233.5 \\
\hline IgM heavy chain variable region [Homo sapiens] & gil4100376 & 13435.7 \\
\hline IgM light chain [Homo sapiens] & gi|4261790 & 14170.1 \\
\hline $\begin{array}{l}\text { Shigella dysenteriae type 1-specific antibody light chain } \\
\text { variable region [Mus sp.] }\end{array}$ & gi|1087011 & 12209.2 \\
\hline $\begin{array}{l}\text { This CDS feature is included to show the translation of } \\
\text { the corresponding V_region. Presenttly translation } \\
\text { qualifiers on V_region features are illegal [Homo } \\
\text { sapiens] }\end{array}$ & gi|619634 & 15022.5 \\
\hline Unknown (protein for MGC:27152) [Homo sapiens] & gil16198375 & 26910.5 \\
\hline Unknown (protein for MGC:31936) [Homo sapiens] & gi|18044241 & 26886.3 \\
\hline Unknown (protein for MGC:31944) [Homo sapiens] & gi|18380972 & 26874.3 \\
\hline amyloid fibril protein Es492,Ig lambda & gi|224730 & 19163.6 \\
\hline $\begin{array}{l}\text { amyloid lambda } 6 \text { light chain variable region SAR } \\
\text { [Homo sapiens] }\end{array}$ & gi|14279407 & 13099.4 \\
\hline $\begin{array}{l}\text { amyloidogenic immunoglobulin lambda VI chain } \\
\text { variable region [Homo sapiens] }\end{array}$ & gi|4732060 & 12825.2 \\
\hline $\begin{array}{l}\text { anti-HIV-1 gp } 120 \text { immunoglobulin } 23 e \text { lambda light } \\
\text { chain [Homo sapiens] }\end{array}$ & gi|40647140 & 21791.8 \\
\hline anti-Rh(D) antibody [Homo sapiens] & gil77765075 & 22710.4 \\
\hline $\begin{array}{l}\text { anti-carcinoma surface antigen monoclonal antibody } \\
\text { AC6C3 [Homo sapiens] }\end{array}$ & gi|998391 & 15218.5 \\
\hline anti-flavocytochrome b2 IgG [Mus musculus] & gil12584580 & 26584.3 \\
\hline $\begin{array}{l}\text { anti-peptide/MHC complex HLA-A1/MAGE-A1 } \\
\text { monoclonal antibody light chain [Homo sapiens] }\end{array}$ & gil16974104 & 24982.5 \\
\hline $\begin{array}{l}\text { anti-rabies SO57 immunoglobulin lambda light chain } \\
\text { [Homo sapiens] }\end{array}$ & gi|27728683 & 27766.8 \\
\hline $\begin{array}{l}\text { anti-rabies SOJB immunoglobulin lambda light chain } \\
\text { [Homo sapiens] }\end{array}$ & gi|27728687 & 26882.4 \\
\hline $\begin{array}{l}\text { antiporphyrin immunoglobulin } \mathrm{G} \text { lambda chain variable } \\
\text { region [Mus musculus] }\end{array}$ & gi|27763668 & 25955.1 \\
\hline $\begin{array}{l}\text { antiporphyrin immunoglobulin } \mathrm{G} \text { lambda chain variable } \\
\text { region [Mus musculus] }\end{array}$ & gi|27762580 & 26098.1 \\
\hline mmunoglobin anti-granzymeB light chain variable & gi|13235108 & \\
\hline
\end{tabular}


region [Homo sapiens]

immunoglobulin kappa light chain $\mathrm{V}$ region [Homo

gi|2599538

13492.9

sapiens

gi|2599540

12635.3

immunoglobulin kappa light chain variable region DPL5 gil5731247

15163.6

immunoglobulin lambda chain [Macaca mulatta] $\quad$ gi|40287807

15247.7

immunoglobulin lambda chain [Macaca mulatta] gi|40287765

15392.8

immunoglobulin lambda chain [Macaca mulatta]

gi|40287823 $\quad 14669.2$

immunoglobulin lambda chain [Macaca mulatta]

gi|40287763

15350.7

immunoglobulin lambda chain [Macaca mulatta]

gi|40287819 15406.7

immunoglobulin lambda chain [Macaca mulatta]

gi|40287821 14758.2

immunoglobulin lambda chain [Macaca mulatta]

gi|40287769 $\quad 15283.7$

immunoglobulin lambda chain [Macaca mulatta]

gil40287813 $\quad 15048.6$

immunoglobulin lambda chain [Macaca mulatta]

gi|40287815 15018.6

immunoglobulin lambda chain [Macaca mulatta]

gil40287817 $\quad 14909.5$

immunoglobulin lambda chain [Macaca mulatta]

gil40287771 $\quad 15345.6$

immunoglobulin lambda chain [Macaca mulatta]

gi|40287809 15203.7

immunoglobulin lambda chain [Macaca mulatta]

gil40287827 $\quad 15006.6$

immunoglobulin lambda chain [Macaca mulatta]

gi|40287851 14125.2

immunoglobulin lambda chain [Macaca mulatta]

gi|40287837 $\quad 14169.2$

immunoglobulin lambda chain [Macaca mulatta]

14185.3

immunoglobulin lambda chain [Macaca mulatta]

gi|40287767 15461.6

immunoglobulin lambda chain [Macaca mulatta]

gi|40287847 14155.2

immunoglobulin lambda chain [Macaca mulatta]

gi|40287849 $\quad 14155.2$

immunoglobulin lambda chain [Macaca mulatta]

gi|40287773 15436.7

immunoglobulin lambda chain [Macaca mulatta]

gil40287825 14857.4

immunoglobulin lambda chain [Macaca mulatta]

gi|40287833

14161

immunoglobulin lambda chain [Macaca mulatta]

gi|40287843

14169.2 


$\begin{array}{llc}\text { immunoglobulin lambda chain [Macaca mulatta] } & \text { gi|40287841 } & 14155.2 \\ \text { immunoglobulin lambda chain [Macaca mulatta] } & \text { gi|40287811 } & 15240.6 \\ \text { immunoglobulin lambda chain [Macaca mulatta] } & \text { gi|40287845 } & 14203.2 \\ \text { immunoglobulin lambda chain [Macaca mulatta] } & \text { gi|40287831 } & 14283.2 \\ \text { immunoglobulin lambda chain [Macaca mulatta] } & \text { gi|40287803 } & 15296.7 \\ \text { immunoglobulin lambda chain [Macaca mulatta] } & \text { gi|40287829 } & 14309.2 \\ \text { immunoglobulin lambda chain [Macaca mulatta] } & \text { gi|40287835 } & 14199.3 \\ \text { immunoglobulin lambda chain [Mus musculus] } & \text { gi|393152 } & 12729.5 \\ \text { immunoglobulin lambda chain [Mus musculus] } & \text { gi|37196479 } & 25204.5 \\ \text { immunoglobulin lambda chain [Mus musculus] } & \text { gi|37196529 } & 25111.5 \\ \text { immunoglobulin lambda chain [Mus musculus] } & \text { gi|393148 } & 12644.4 \\ \text { immunoglobulin lambda chain [Mus musculus] } & \text { gi|37196444 } & 25085.4 \\ \text { immunoglobulin lambda chain [Mus musculus] } & \text { gi|37196446 } & 24931.4\end{array}$




$\begin{array}{llc}\text { immunoglobulin lambda chain [Mus musculus] } & \text { gi|37196494 } & 25188.7 \\ \text { immunoglobulin lambda chain [Mus musculus] } & \text { gi|37196485 } & 25089.5 \\ \text { immunoglobulin lambda chain [Mus musculus] } & \text { gi|37196498 } & 25077.5 \\ \text { immunoglobulin lambda chain [Mus musculus] } & \text { gi|37196440 } & 25026.4 \\ \text { immunoglobulin lambda chain [Mus musculus] } & \text { gi|37196527 } & 25040.4 \\ \text { immunoglobulin lambda chain [Mus musculus] } & \text { gi|37196508 } & 25095.6 \\ \text { immunoglobulin lambda chain [Mus musculus] } & \text { gi|37196512 } & 25133.6 \\ \text { immunoglobulin lambda chain [Mus musculus] } & \text { gi|37221117 } & 25075.4 \\ \text { immunoglobulin lambda chain [Mus musculus] } & \text { gi|37196481 } & 25056.6 \\ \text { immunoglobulin lambda chain [Mus musculus] } & \text { gi|37196514 } & 25130.5 \\ \text { immunoglobulin lambda chain [Mus musculus] } & \text { gi|37196504 } & 25009.4 \\ \text { immunoglobulin lambda chain [Mus musculus] } & \text { gi|37196467 } & 25154.6 \\ \text { immunoglobulin lambda chain [Mus musculus] } & \text { gi|37221087 } & 25117.6\end{array}$




\begin{tabular}{|c|c|c|}
\hline immunoglobulin lambda chain [Mus musculus] & gi|37221135 & 25131.6 \\
\hline immunoglobulin lambda chain [Mus musculus] & gi|37221145 & 25000.5 \\
\hline immunoglobulin lambda chain [Mus musculus] & gi|37221137 & 25075.5 \\
\hline immunoglobulin lambda chain [Mus musculus] & gi|37221113 & 25027.6 \\
\hline immunoglobulin lambda chain [Mus musculus] & gi|37221155 & 25117.5 \\
\hline immunoglobulin lambda chain [Mus musculus] & gi|37221167 & 25164.5 \\
\hline immunoglobulin lambda chain [Mus musculus] & gi|37221127 & 25101.6 \\
\hline immunoglobulin lambda chain [Mus musculus] & gi|37221115 & 25088.6 \\
\hline immunoglobulin lambda chain [Mus musculus] & gi|37196531 & 25138.5 \\
\hline immunoglobulin lambda chain [Mus musculus] & gi|37196496 & 25106.5 \\
\hline $\begin{array}{l}\text { immunoglobulin lambda chain variable and joining } \\
\text { regions [Mus musculus] }\end{array}$ & gi|623386 & 12824.5 \\
\hline $\begin{array}{l}\text { immunoglobulin lambda chain variable and joining } \\
\text { regions [Mus musculus] }\end{array}$ & gi|619963 & 12757.4 \\
\hline $\begin{array}{l}\text { immunoglobulin lambda chain variable region [Homo } \\
\text { sapiens] }\end{array}$ & gi|5578834 & 12793.3 \\
\hline $\begin{array}{l}\text { immunoglobulin lambda chain variable region [Homo } \\
\text { sapiens] }\end{array}$ & gi|587378 & 13906.9 \\
\hline $\begin{array}{l}\text { immunoglobulin lambda chain variable region [Homo } \\
\text { sapiens] }\end{array}$ & gi|587360 & 13715.8 \\
\hline $\begin{array}{l}\text { immunoglobulin lambda chain variable region [Homo } \\
\text { sapiens] }\end{array}$ & gi|587388 & 14077 \\
\hline $\begin{array}{l}\text { immunoglobulin lambda chain variable region [Homo } \\
\text { sapiens] }\end{array}$ & gi|587416 & 14177 \\
\hline $\begin{array}{l}\text { immunoglobulin lambda chain variable region [Homo } \\
\text { sapiens] }\end{array}$ & gi|587384 & 14013.9 \\
\hline $\begin{array}{l}\text { immunoglobulin lambda chain variable region [Homo } \\
\text { sapiens] }\end{array}$ & gi|587398 & 13734.9 \\
\hline $\begin{array}{l}\text { immunoglobulin lambda chain variable region [Homo } \\
\text { sapiens] }\end{array}$ & gi|587358 & 13772.9 \\
\hline $\begin{array}{l}\text { immunoglobulin lambda chain variable region [Homo } \\
\text { sapiens] }\end{array}$ & gi|587402 & 13598.8 \\
\hline $\begin{array}{l}\text { immunoglobulin lambda chain variable region [Homo } \\
\text { sapiens] }\end{array}$ & gi|587412 & 14255.9 \\
\hline $\begin{array}{l}\text { immunoglobulin lambda chain variable region [Homo } \\
\text { sapiens] }\end{array}$ & gi|587408 & 14243.9 \\
\hline $\begin{array}{l}\text { immunoglobulin lambda chain variable region [Homo } \\
\text { sapiens] }\end{array}$ & gi|587414 & 14181.9 \\
\hline $\begin{array}{l}\text { immunoglobulin lambda chain variable region [Homo } \\
\text { sapiens] }\end{array}$ & gi|587395 & 13885 \\
\hline immunoglobulin lambda chain variable regi & gi|587382 & 13693.7 > \\
\hline
\end{tabular}




\begin{tabular}{|c|c|c|}
\hline $\begin{array}{l}\text { immunoglobulin lambda chain variable region [Homo } \\
\text { sapiens] }\end{array}$ & gi|587364 & 13915.9 \\
\hline $\begin{array}{l}\text { immunoglobulin lambda chain variable region [Homo } \\
\text { sapiens] }\end{array}$ & gi|587380 & 14179 \\
\hline $\begin{array}{l}\text { immunoglobulin lambda chain variable region [Homo } \\
\text { sapiens] }\end{array}$ & gi|587404 & 13820 \\
\hline $\begin{array}{l}\text { immunoglobulin lambda chain variable region [Homo } \\
\text { sapiens] }\end{array}$ & gi|587406 & 13840.8 \\
\hline $\begin{array}{l}\text { immunoglobulin lambda chain variable region [Homo } \\
\text { sapiens] }\end{array}$ & gi|587418 & 14481.1 \\
\hline $\begin{array}{l}\text { immunoglobulin lambda chain variable region [Homo } \\
\text { sapiens] }\end{array}$ & gi|587420 & 14617.1 \\
\hline $\begin{array}{l}\text { immunoglobulin lambda chain variable region [Homo } \\
\text { sapiens] }\end{array}$ & gi|587368 & 13590.7 \\
\hline $\begin{array}{l}\text { immunoglobulin lambda chain variable region [Homo } \\
\text { sapiens] }\end{array}$ & gi|587410 & 14198.9 \\
\hline $\begin{array}{l}\text { immunoglobulin lambda chain variable region [Homo } \\
\text { sapiens] }\end{array}$ & gi|587400 & 14112.1 \\
\hline $\begin{array}{l}\text { immunoglobulin lambda light chain VLJ region [Homo } \\
\text { sapiens] }\end{array}$ & gi|21669669 & 30256.8 \\
\hline $\begin{array}{l}\text { immunoglobulin lambda light chain VLJ region [Homo } \\
\text { sapiens] }\end{array}$ & gi|21669665 & 30175.9 \\
\hline $\begin{array}{l}\text { immunoglobulin lambda light chain VLJ region [Homo } \\
\text { sapiens] }\end{array}$ & gi|21669501 & 30537.3 \\
\hline $\begin{array}{l}\text { immunoglobulin lambda light chain VLJ region [Homo } \\
\text { sapiens] }\end{array}$ & gi|21669677 & 30251.9 \\
\hline $\begin{array}{l}\text { immunoglobulin lambda light chain VLJ region [Homo } \\
\text { sapiens] }\end{array}$ & gi|21669243 & 14016 \\
\hline $\begin{array}{l}\text { immunoglobulin lambda light chain VLJ region [Homo } \\
\text { sapiens] }\end{array}$ & gi|21669537 & 30567.2 \\
\hline $\begin{array}{l}\text { immunoglobulin lambda light chain VLJ region [Homo } \\
\text { sapiens] }\end{array}$ & gi|21669521 & 30351.1 \\
\hline $\begin{array}{l}\text { immunoglobulin lambda light chain VLJ region [Homo } \\
\text { sapiens] }\end{array}$ & gi|21669511 & 30644.2 \\
\hline $\begin{array}{l}\text { immunoglobulin lambda light chain VLJ region [Homo } \\
\text { sapiens] }\end{array}$ & gi|21669533 & 30618.2 \\
\hline $\begin{array}{l}\text { immunoglobulin lambda light chain VLJ region [Homo } \\
\text { sapiens] }\end{array}$ & gi|21669643 & 30722.3 \\
\hline $\begin{array}{l}\text { immunoglobulin lambda light chain VLJ region [Homo } \\
\text { sapiens] }\end{array}$ & gi|21669619 & 29766.8 \\
\hline $\begin{array}{l}\text { immunoglobulin lambda light chain VLJ region [Homo } \\
\text { sapiens] }\end{array}$ & gi|21669595 & 30373 \\
\hline $\begin{array}{l}\text { immunoglobulin lambda light chain VLJ region [Homo } \\
\text { sapiens] }\end{array}$ & gi|21669573 & 30091.9 \\
\hline $\begin{array}{l}\text { immunoglobulin lambda light chain VLJ region [Homo } \\
\text { sapiens] }\end{array}$ & gi|21669645 & 30601.2 \\
\hline $\begin{array}{l}\text { immunoglobulin lambda light chain VLJ region [Homo } \\
\text { sapiens] }\end{array}$ & gi|21669505 & 30517.3 \\
\hline $\begin{array}{l}\text { immunoglobulin lambda light chain VLJ region [Homo } \\
\text { sapiens] }\end{array}$ & gi|21669529 & 30366.1 \\
\hline mmunoglobulin lambda light chain VLJ region [Hon & gi|21669495 & 717.4 \\
\hline
\end{tabular}


immunoglobulin lambda light chain VLJ region [Homo gi|21669641 immunoglobulin lambda light chain VLJ region [Homo gi|21669509
sapiens]

immunoglobulin lambda light chain VLJ region [Homo gi|21669515 sapiens]

immunoglobulin lambda light chain VLJ region [Homo $\quad$ gi|21669639
sapiens] immunoglobulin lambda light chain VLJ region [Homo gi|21669493

immunoglobulin lambda light chain VLJ region [Homo gi|21669547
sapiens]

immunoglobulin lambda light chain VLJ region [Homo gi|21669615

immunoglobulin lambda light chain VLJ region [Homo gi|21669587

sapiens

immunoglobulin lambda light chain VLJ region [Homo gi|21669623

immunoglobulin lambda light chain VLJ region [Homo gi|21669599

30191.8

immunoglobulin lambda light chain VLJ region [Homo gi|21669575

sapiens]

immunoglobulin lambda light chain VLJ region [Homo gi|21669525

immunoglobulin lambda light chain VLJ region [Homo gi|21669545 sapiens]

immunoglobulin lambda light chain VLJ region [Homo gil21669581

30673.3

immunoglobulin lambda light chain VLJ region [Homo gi|21669593

immunoglobulin lambda light chain VLJ region [Homo gi|21669597 sapiens]

immunoglobulin lambda light chain VLJ region [Homo gi|21669671 30264.9 30318 30021.7 immunoglobulin lambda light chain VLJ region [Homo gi|21669551

immunoglobulin lambda light chain VLJ region [Homo gi|21669675
sapiens] immunoglobulin lambda light chain VLJ region [Homo gi|21669543

immunoglobulin lambda light chain VLJ region [Homo gi|21669655 sapiens]

immunoglobulin lambda light chain VLJ region [Homo gi|21669517 
immunoglobulin lambda light chain VLJ region [Homo gi|21669617

immunoglobulin lambda light chain VLJ region [Homo gi|21669629 sapiens

immunoglobulin lambda light chain VLJ region [Homo gi|21669607 sapiens]

immunoglobulin lambda light chain VLJ region [Homo $\quad$ gi|21669609
sapiens]

immunoglobulin lambda light chain VLJ region [Homo gi|21669539
sapiens]

immunoglobulin lambda light chain VLJ region [Homo gi|21669531

immunoglobulin lambda light chain VLJ region [Homo gi|21669563

immunoglobulin lambda light chain VLJ region [Homo gi|21669567 30375.1

immunoglobulin lambda light chain VLJ region [Homo gi|21669247 sapiens]

immunoglobulin lambda light chain VLJ region [Homo gi|21669653 sapiens]

immunoglobulin lambda light chain VLJ region [Homo $\quad$ gi|21669627
sapiens] immunoglobulin lambda light chain VLJ region [Homo gi|21669603 sapiens] immunoglobulin lambda light chain VLJ region [Homo gi|21669601
sapiens]

immunoglobulin lambda light chain VLJ region [Homo gi|21669541 sapiens

immunoglobulin lambda light chain VLJ region [Homo gi|21669565 sapiens

immunoglobulin lambda light chain VLJ region [Homo gi|21669667 12858.3

31564.9

29857.8 
sapiens]

\begin{tabular}{|c|c|c|}
\hline immunoglobulin lambda light chain [Homo sapiens] & gi|2765427 & 27177.5 \\
\hline immunoglobulin lambda light chain [Homo sapiens] & $\mathrm{gi} \mid 219888$ & 27688 \\
\hline immunoglobulin lambda light chain [Homo sapiens] & gi|21311293 & 15402.9 \\
\hline immunoglobulin lambda light chain [Homo sapiens] & gil468247 & 24988.4 \\
\hline immunoglobulin lambda light chain [Homo sapiens] & gi|6467839 & 25015.6 \\
\hline immunoglobulin lambda light chain [Homo sapiens] & gil1684928 & 22607.3 \\
\hline $\begin{array}{l}\text { immunoglobulin lambda light chain variable and } \\
\text { constant region [Homo sapiens] }\end{array}$ & gi|27818835 & 12711.3 \\
\hline $\begin{array}{l}\text { immunoglobulin lambda light chain variable region 4a } \\
\text { [Homo sapiens] }\end{array}$ & gi|3335588 & 13423.7 \\
\hline $\begin{array}{l}\text { immunoglobulin lambda light chain variable region 4a } \\
\text { [Homo sapiens] }\end{array}$ & gil|3335580 & 13977 \\
\hline $\begin{array}{l}\text { immunoglobulin lambda light chain variable region 4a } \\
\text { [Homo sapiens] }\end{array}$ & gi|3335594 & 13307.6 \\
\hline $\begin{array}{l}\text { immunoglobulin lambda light chain variable region 4a } \\
\text { [Homo sapiens] }\end{array}$ & gi|3335586 & 13472.6 \\
\hline $\begin{array}{l}\text { immunoglobulin lambda light chain variable region } \\
\text { [Homo sapiens] }\end{array}$ & gi|47271324 & 12792.4 \\
\hline $\begin{array}{l}\text { immunoglobulin lambda light chain variable region } \\
\text { [Homo sapiens] }\end{array}$ & gi|47271328 & 12809.3 \\
\hline $\begin{array}{l}\text { immunoglobulin lambda light chain variable region } \\
\text { [Homo sapiens] }\end{array}$ & gi|3388057 & 13025.4 \\
\hline $\begin{array}{l}\text { immunoglobulin lambda light chain variable region } \\
\text { [Homo sapiens] }\end{array}$ & gi|4324232 & 12255.3 \\
\hline $\begin{array}{l}\text { immunoglobulin lambda light chain variable region } \\
\text { [Homo sapiens] }\end{array}$ & gi|4324110 & 12176 \\
\hline $\begin{array}{l}\text { immunoglobulin lambda light chain variable region } \\
\text { [Homo sapiens] }\end{array}$ & gi|4324062 & 11591.7 \\
\hline $\begin{array}{l}\text { immunoglobulin lambda light chain variable region } \\
\text { [Homo sapiens] }\end{array}$ & gi|3388053 & 13116.5 \\
\hline $\begin{array}{l}\text { immunoglobulin lambda light chain variable region } \\
\text { [Homo sapiens] }\end{array}$ & gi|4324186 & 12346.3 \\
\hline $\begin{array}{l}\text { immunoglobulin lambda light chain variable region } \\
\text { [Homo sapiens] }\end{array}$ & gi|4324128 & 12202.1 \\
\hline $\begin{array}{l}\text { immunoglobulin lambda light chain variable region } \\
\text { [Homo sapiens] }\end{array}$ & gi|4324088 & 11406.7 \\
\hline $\begin{array}{l}\text { immunoglobulin lambda light chain variable region } \\
\text { [Homo sapiens] }\end{array}$ & gi|4324044 & 12366.1 \\
\hline $\begin{array}{l}\text { immunoglobulin lambda light chain variable region } \\
\text { [Homo sapiens] }\end{array}$ & gil4324178 & 12060 \\
\hline $\begin{array}{l}\text { immunoglobulin lambda light chain variable region } \\
\text { [Homo sapiens] }\end{array}$ & gil3388059 & 13262.6 \\
\hline $\begin{array}{l}\text { immunoglobulin lambda light chain variable region } \\
\text { [Homo sapiens] }\end{array}$ & gil4324056 & 12062.1 \\
\hline immunoglobulin lambda light chain variable region & gi|4324244 & 12626. \\
\hline
\end{tabular}


[Homo sapiens]

immunoglobulin lambda light chain variable region

gi|3388063

munoglobulin lambda light chain variable region

gi|3388075

13079.6

mmunoglobulin lambda light chain variable region

gi|27369054

15208.4

immunoglobulin lambda light chain variable region

gi|4324152

2537.3

immunoglobulin lambda light chain variable region [Homo sapiens]

gi|4324040 12512.4

immunoglobulin lambda light chain variable region
[Homo sapiens]

gi|4324200 11772

immunoglobulin lambda light chain variable region
[Homo sapiens]

gi|4324098 $\quad 12517.2$

immunoglobulin lambda light chain variable region gi|4324026 $\quad 12285.1$

immunoglobulin lambda light chain variable region

gi|4324140 $\quad 11872$

immunoglobulin lambda light chain variable region

gi|4324102 $\quad 11790.8$

immunoglobulin lambda light chain variable region

gi|4324230 $\quad 11932$

immunoglobulin lambda light chain variable region
[Homo gapiens]

gi|27369050

12756.3

immunoglobulin lambda light chain variable region

gil4324268

12233.1

immunoglobulin lambda light chain variable region

gi|27369034 $\quad 12963$.

[Homo sapiens]

gi|4324096 11960

immunoglobulin lambda light chain variable region
[Homo sapiens]

immunoglobulin lambda light chain variable region
[Homo sapiens]

gi|4324124

12315.2

immunoglobulin lambda light chain variable region

gil4324078

2841.4

immunoglobulin lambda light chain variable region [Homo sapiens]

gi|4324252 12056

immunoglobulin lambda light chain variable region gil4324142 11873

immunoglobulin lambda light chain variable region

[Homo sapiens]

immunoglobulin lambda light chain variable region gi|27369083 $\quad 15745.7$

immunoglobulin lambda light chain variable region [Homo sapiens]

gi|3388061 $\quad 13160.6$

gil4324164 $\quad 12846.4$

gi|4324262 11933.8

immunoglobulin lambda light chain variable region
[Homo sapiens]

immunoglobulin lambda light chain variable region

gil4324282 $\quad 11883.8$

immunoglobulin lambda light chain variable region
[Homo sapiens]

gi|4324280 $\quad 12134$

immunoglobulin lambda light chain variable region

gi|4324204 $\quad 11849.9$ 
[Homo sapiens]

immunoglobulin lambda light chain variable region

gi|27369065 14920

munoglobulin lambda light chain variable region

gi|4324144

12083

mmunoglobulin lambda light chain variable region

gi|4324246 $\quad 12210$

[Homo sapiens]

gi|4324038 $\quad 11924$

immunoglobulin lambda light chain variable region
[Homo sapiens]

gi|3388049 $\quad 13330.8$

immunoglobulin lambda light chain variable region
[Homo sapiens]

immunoglobulin lambda light chain variable region
[Homo sapiens]

gi|4324028 $\quad 13021$

immunoglobulin lambda light chain variable region
[Homo sapiens]

gi|4324154 12044

immunoglobulin lambda light chain variable region gi|4324050 $\quad 12074$

immunoglobulin lambda light chain variable region

gi|4324190 $\quad 12568.1$

[Homo sapiens]

gi|4324196 11833.8

immunoglobulin lambda light chain variable region
[Homo sapiens]

immunoglobulin lambda light chain variable region

gi|4324266 $\quad 12386$

immunoglobulin lambda light chain variable region

gi|4324116 $\quad 12722$

immunoglobulin lambda light chain variable region gi|3388073 $\quad 12875$

immunoglobulin lambda light chain variable region

[Homo sapiens]

gi|4324080 $\quad 10741.5$

gi|4324046 12364.2

immunoglobulin lambda light chain variable region
[Homo sapiens]

immunoglobulin lambda light chain variable region

gi|4324284 $\quad 12283.2$

[Homo sapiens]

gi|4324260 $\quad 12473$

gil4324174 12252.3

immunoglobulin lambda light chain variable region
[Homo sapiens]

immunoglobulin lambda light chain variable region

10632

immunoglobulin lambda light chain variable region

[Homo sapiens]

gi|4324224 12457.

immunoglobulin lambda light chain variable region
[Homo sapiens]

gi|4324270 $\quad 11837.9$

immunoglobulin lambda light chain variable region

gi|4324172 $\quad 12429.1$

gi|4324030 12063.2

immunoglobulin lambda light chain variable region
[Homo sapiens]

immunoglobulin lambda light chain variable region

gil4324092 $\quad 11817,7$

immunoglobulin lambda light chain variable region

immunoglobulin lambda light chain variable region

gi|4324064 12023 
[Homo sapiens]

immunoglobulin lambda light chain variable region

gil4324272 122062

immunoglobulin lambda light chain variable region
[Homo sapiens]

gi|4324162 12193.

immunoglobulin lambda light chain variable region

gi|3388069 $\quad 12948.3$

immunoglobulin lambda light chain variable region

gi|4324254 12263

immunoglobulin lambda light chain variable region
[Homo sapiens]

gi|4324042

11677.8

immunoglobulin lambda light chain variable region

gi|4324248

11989.9

immunoglobulin lambda light chain variable region
[Homo sapiens]

gi|4324054 12122

immunoglobulin lambda light chain variable region
[Homo sapiens]

gi|4324122 $\quad 12453.2$

immunoglobulin lambda light chain variable region

gi|27369069 $\quad 15365$

gi|47271322 13873.

immunoglobulin lambda light chain variable region
[Homo sapiens]

immunoglobulin lambda-1 light chain precursor [Mus

gi|387376

gil185364

27123.5

immunoglobulin lambda-chain [Homo sapiens]

gi|434698

15026.4

immunoglobulin lambda-chain [Homo sapiens]

gil186135

16522.3

immunoglobulin lambda-chain [Homo sapiens]

gi|186083

15465.5

immunoglobulin lambda-chain [Homo sapiens] $\quad$ gi|186091 $\quad 14936.3$

immunoglobulin lambda-chain [Homo sapiens] $\quad$ gil186093 $\quad 15096.4$

immunoglobulin lambda-chain [Homo sapiens] $\quad$ gil186095

immunoglobulin lambda-chain [Mus musculus] $\quad$ gil197685 $\quad 14931.8$

immunoglobulin lambda-chain [Mus musculus] $\quad$ gi|197683 $\quad 14849.6$

immunoglobulin light chain Fab fragment [Homo $\quad$ gil3954950 14179

immunoglobulin light chain [Homo sapiens] $\quad$ gi|553496 $\quad 15735.8$

immunoglobulin light chain [Homo sapiens] $\quad$ gil1684927 $\quad 23162.6$

immunoglobulin light chain [Mus musculus] $\quad$ gil34481886 23062.4

immunoglobulin light chain lambda 1 [Homo sapiens] $\quad$ gil11992192 14157.

immunoglobulin light chain lambda 2 [Homo sapiens] $\quad$ gi|11992186 $\quad 13981.7$ 


\begin{tabular}{|c|c|c|}
\hline immunoglobulin light chain lambda 3 [Homo sapiens] & gi|11992196 & 13870.9 \\
\hline immunoglobulin light chain lambda 6 [Homo sapiens] & gi|11992204 & 14329 \\
\hline immunoglobulin light chain lambda 6 [Homo sapiens] & gi|11992202 & 14123.9 \\
\hline immunoglobulin light chain lambda 6 [Homo sapiens] & gi|11992200 & 14482.2 \\
\hline $\begin{array}{l}\text { immunoglobulin light chain variable region [Homo } \\
\text { sapiens] }\end{array}$ & gil11558190 & 14416.3 \\
\hline $\begin{array}{l}\text { immunoglobulin light chain variable region [Homo } \\
\text { sapiens] }\end{array}$ & gi|12734074 & 13701.6 \\
\hline $\begin{array}{l}\text { immunoglobulin light chain variable region [Homo } \\
\text { sapiens] }\end{array}$ & gi|12734067 & 13965 \\
\hline $\begin{array}{l}\text { immunoglobulin light chain variable region [Homo } \\
\text { sapiens] }\end{array}$ & gi|12734092 & 14357.1 \\
\hline $\begin{array}{l}\text { immunoglobulin light chain variable region [Homo } \\
\text { sapiens] }\end{array}$ & gi|11121491 & 14551.4 \\
\hline $\begin{array}{l}\text { immunoglobulin light chain variable region [Homo } \\
\text { sapiens] }\end{array}$ & gi|11121493 & 14528.5 \\
\hline $\begin{array}{l}\text { immunoglobulin light chain variable region [Homo } \\
\text { sapiens] }\end{array}$ & gi|22095259 & 14951.8 \\
\hline $\begin{array}{l}\text { immunoglobulin light chain variable region [Homo } \\
\text { sapiens] }\end{array}$ & gi|18025610 & 16551.1 \\
\hline $\begin{array}{l}\text { immunoglobulin light chain variable region [Homo } \\
\text { sapiens] }\end{array}$ & gi|22095217 & 13432.6 \\
\hline $\begin{array}{l}\text { immunoglobulin light chain variable region [Homo } \\
\text { sapiens] }\end{array}$ & gi|22095251 & 12732.3 \\
\hline $\begin{array}{l}\text { immunoglobulin light chain variable region [Homo } \\
\text { sapiens] }\end{array}$ & gi|3327990 & 14795.4 \\
\hline $\begin{array}{l}\text { immunoglobulin light chain variable region [Homo } \\
\text { sapiens] }\end{array}$ & gi|7688925 & 12809.5 \\
\hline $\begin{array}{l}\text { immunoglobulin light chain variable region [Homo } \\
\text { sapiens] }\end{array}$ & gi|3327988 & 14945.5 \\
\hline $\begin{array}{l}\text { immunoglobulin light chain variable region [Homo } \\
\text { sapiens] }\end{array}$ & gi|3327992 & 14693.2 \\
\hline $\begin{array}{l}\text { immunoglobulin light chain variable region [Homo } \\
\text { sapiens] }\end{array}$ & gi|22095229 & 12736.4 \\
\hline $\begin{array}{l}\text { immunoglobulin light chain variable region [Homo } \\
\text { sapiens] }\end{array}$ & gi|3328010 & 14432.5 \\
\hline $\begin{array}{l}\text { immunoglobulin light chain variable region [Homo } \\
\text { sapiens] }\end{array}$ & gi|22095189 & 12931.4 \\
\hline $\begin{array}{l}\text { immunoglobulin light chain variable region [Homo } \\
\text { sapiens] }\end{array}$ & gi|12698680 & 13614.8 \\
\hline $\begin{array}{l}\text { immunoglobulin light chain variable region [Homo } \\
\text { sapiens] }\end{array}$ & gi|22095253 & 15061.6 \\
\hline $\begin{array}{l}\text { immunoglobulin light chain variable region [Homo } \\
\text { sapiens] }\end{array}$ & gil 465170 & 17501.8 \\
\hline $\begin{array}{l}\text { immunoglobulin light chain variable region [Homo } \\
\text { sapiens] }\end{array}$ & gi|12698674 & 14424.1 \\
\hline immunoglobulin light chain variable region [Hom & gil 1087002 & 12240.2 \\
\hline
\end{tabular}


immunoglobulin light chain variable region [Homo

gi|12698676 14601.2 immunoglobulin light chain variable region [Homo
sapiens] gi|22095209 12737.3

immunoglobulin light chain variable region [Homo
sapiens] immunoglobulin light chain variable region [Homo
sapiens] immunoglobulin light chain variable region [Mus
musculus] immunoglobulin variable region [Homo sapiens] gi|3327994 $\quad 14805.5$ gi|465176 $\quad 17312.9$ gi|37784142 $\quad 14451.3$ gi|306918 $\quad 15384.7$ lambda 1 immunoglobin light chain variable region
[Homo sapiens] gi|5524117 $\quad 14477$

lambda 1 immunoglobin light chain variable region gi|15524109 13891.7

lambda 1 immunoglobin light chain variable region [Homo sapiens] gi|5524095 $\quad 14282.2$ lambda 1 immunoglobin light chain variable region
[Homo sapiens] gi|5524093 13955

lambda 1 immunoglobin light chain variable region

lambda 1 immunoglobin light chain variable region [Homo sapiens]

lambda 1 immunoglobin light chain variable region lambda 1 immunoglobin light chain variable region [Homo sapiens]

lambda 1 immunoglobin light chain variable region
[Homo sapiens] lambda 1 immunoglobin light chain variable region lambda 1 immunoglobin light chain variable region
[Homo sapiens]

lambda 1 immunoglobin light chain variable region [Homo sapiens]

lambda 1 immunoglobin light chain variable region gi|5524125 gi|5524091 $\quad 13849.8$ gi|5524131 $\quad 14165.9$ gi|5524121 $\quad 14225.9$ gi|5524123 14359 gil5524115 14219

lambda 1 immunoglobin light chain variable region

gi|5524107 $\quad 13715.9$

gi|5524087 $\quad 13770.9$

lambda 1 immunoglobin light chain variable region
[Homo sapiens]

lambda 1 immunoglobin light chain variable region gi|5524127 
[Homo sapiens]

\begin{tabular}{|c|c|c|}
\hline $\begin{array}{l}\text { lambda } 1 \text { immunoglobulin light chain variable region } \\
\text { [Homo sapiens] }\end{array}$ & gi|3023105 & 13742.8 \\
\hline $\begin{array}{l}\text { lambda } 1 \text { immunoglobulin light chain variable region } \\
\text { [Homo sapiens] }\end{array}$ & gi|3023133 & 14147.8 \\
\hline $\begin{array}{l}\text { lambda } 1 \text { immunoglobulin light chain variable region } \\
\text { [Homo sapiens] }\end{array}$ & gi|3023119 & 14027.1 \\
\hline $\begin{array}{l}\text { lambda } 1 \text { immunoglobulin light chain variable region } \\
\text { [Homo sapiens] }\end{array}$ & gi|3023123 & 14218 \\
\hline $\begin{array}{l}\text { lambda } 1 \text { immunoglobulin light chain variable region } \\
\text { [Homo sapiens] }\end{array}$ & gi|3023113 & 13680 \\
\hline $\begin{array}{l}\text { lambda } 1 \text { immunoglobulin light chain variable region } \\
\text { [Homo sapiens] }\end{array}$ & gi|3023115 & 13947.8 \\
\hline $\begin{array}{l}\text { lambda } 1 \text { immunoglobulin light chain variable region } \\
\text { [Homo sapiens] }\end{array}$ & gi|3023125 & 14149.9 \\
\hline $\begin{array}{l}\text { lambda } 1 \text { immunoglobulin light chain variable region } \\
\text { [Homo sapiens] }\end{array}$ & gi|4761260 & 13789.8 \\
\hline $\begin{array}{l}\text { lambda } 1 \text { immunoglobulin light chain variable region } \\
\text { [Homo sapiens] }\end{array}$ & gi|3023103 & 13900 \\
\hline $\begin{array}{l}\text { lambda } 1 \text { immunoglobulin light chain variable region } \\
\text { [Homo sapiens] }\end{array}$ & gi|3023129 & 14287.9 \\
\hline $\begin{array}{l}\text { lambda } 1 \text { immunoglobulin light chain variable region } \\
\text { [Homo sapiens] }\end{array}$ & gi|4761254 & 13634.7 \\
\hline $\begin{array}{l}\text { lambda } 1 \text { immunoglobulin light chain variable region } \\
\text { [Homo sapiens] }\end{array}$ & gi|3023121 & 14378 \\
\hline $\begin{array}{l}\text { lambda } 2 \text { immunoglobulin light chain variable region } \\
\text { [Homo sapiens] }\end{array}$ & gi|4927958 & 14181.1 \\
\hline $\begin{array}{l}\text { lambda } 2 \text { immunoglobulin light chain variable region } \\
\text { [Homo sapiens] }\end{array}$ & gi|4761264 & 13799.8 \\
\hline $\begin{array}{l}\text { lambda } 3 \text { immunoglobulin light chain variable region } \\
\text { [Homo sapiens] }\end{array}$ & gi|4761270 & 13778.7 \\
\hline $\begin{array}{l}\text { lambda } 3 \text { immunoglobulin light chain variable region } \\
\text { [Homo sapiens] }\end{array}$ & gi|4761268 & 13708.7 \\
\hline $\begin{array}{l}\text { lambda } 6 \text { immunoglobulin light chain variable region } \\
\text { [Homo sapiens] }\end{array}$ & gi|4927956 & 12587.1 \\
\hline $\begin{array}{l}\text { lambda } 6 \text { immunoglobulin light chain variable region } \\
\text { [Homo sapiens] }\end{array}$ & gi|4761278 & 14492 \\
\hline $\begin{array}{l}\text { lambda } 6 \text { immunoglobulin light chain variable region } \\
\text { [Homo sapiens] }\end{array}$ & gi|4761276 & 14367 \\
\hline $\begin{array}{l}\text { lambda } 6 \text { immunoglobulin light chain variable region } \\
\text { [Homo sapiens] }\end{array}$ & gi|4761280 & 13936.7 \\
\hline monoclonal antibody HB4C5 light chain [Homo sapiens & ] gil1524073 & 13428.9 \\
\hline protein CAP,Bence-Jones & gi|223968 & 12540.1 \\
\hline protein MES,Bence-Jones & gi|223971 & 12715.2 \\
\hline protein NIG51 lambda,Bence-Jones & gi|223229 & 24586.2 \\
\hline protein OKA,Bence-Jones & gi|223972 & 12681.2 \\
\hline orotein SUT,Bence-Jones & gi|223863 & 13261.4 \\
\hline
\end{tabular}


variable immunoglobulin anti-steroid light chain [Homo gi|1695139

\section{Peptide Information}

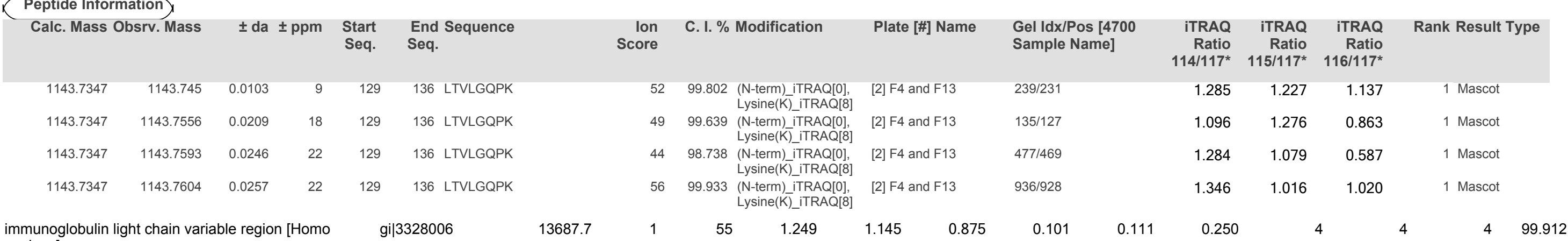

152 immunoglobulin light chain variable region [Homo gil3328006

Protein Group

\section{immunoglobulin lambda light chain variable region
[Homo sapiens] gi|27369079 $\quad 12877$.}

\begin{tabular}{|cccccc}
\hline $\begin{array}{c}\text { Peptide Information } \\
\text { Calc. Mass Obsrv. Mass }\end{array}$ & \pm da \pm ppm & $\begin{array}{l}\text { Start } \\
\text { Seq. }\end{array}$ & $\begin{array}{l}\text { End Sequence } \\
\text { Seq. }\end{array}$ \\
\hline 1143.7347 & 1143.745 & 0.0103 & 9 & 107 & 114 LTVXGQPK \\
1143.7347 & 1143.7556 & 0.0209 & 18 & 107 & 114 LTVXGQPK \\
1143.7347 & 1143.7593 & 0.0246 & 22 & 107 & 114 LTVXGQPK \\
1143.7347 & 1143.7604 & 0.0257 & 22 & 107 & 114 LTVXGQPK \\
\hline
\end{tabular}

153 PREDICTED: EGF containing fibulin-like extracelluter matrix protein 1 [Canis lupus familiaris]

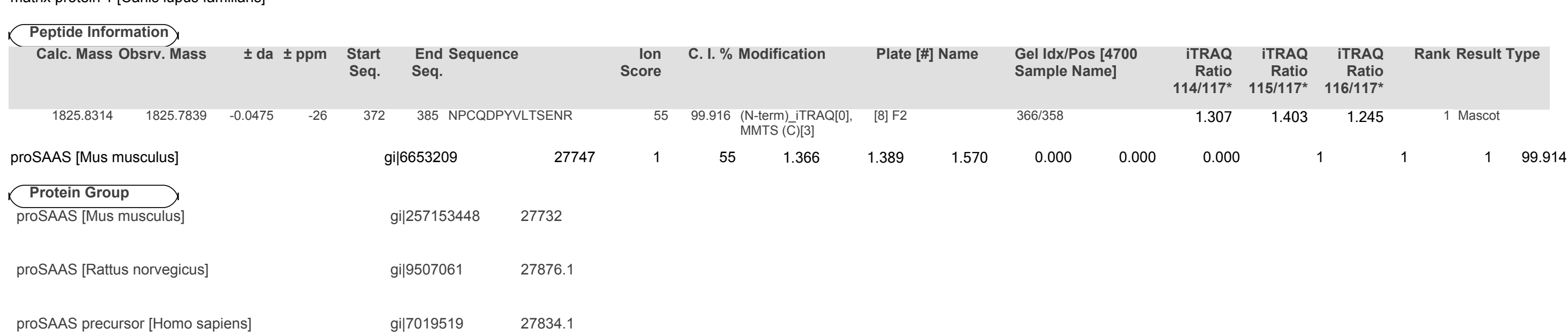




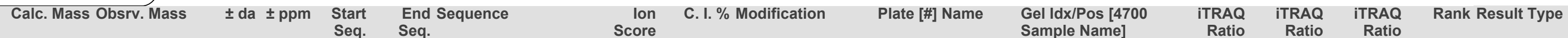
Seq. Seq. Score

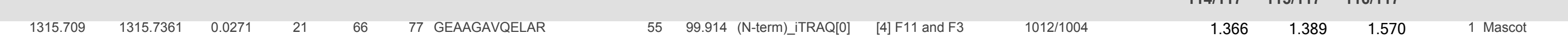

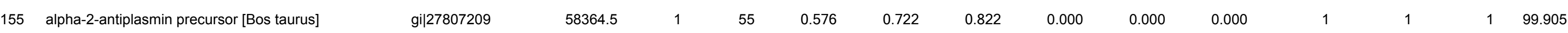
Peptide Information

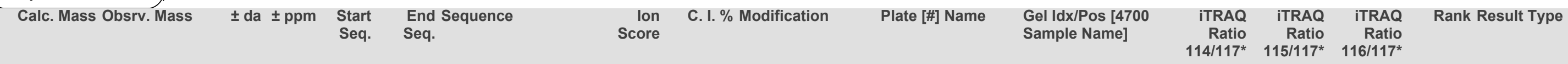

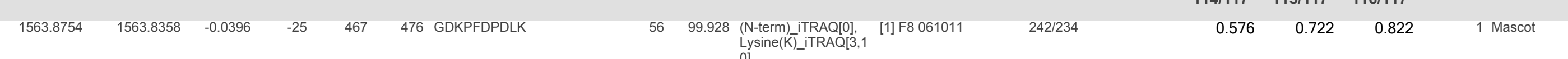

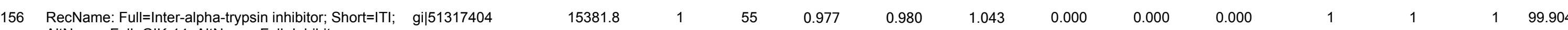

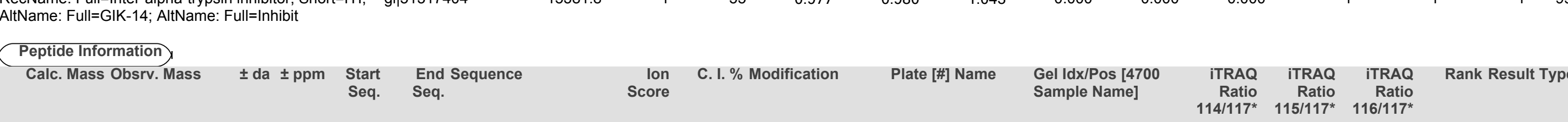

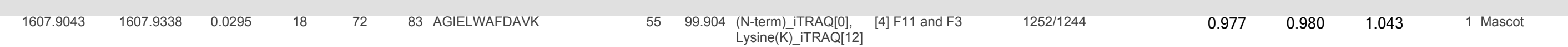

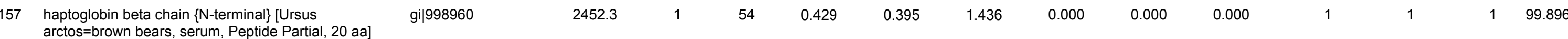

Peptide Information

$\begin{array}{llllllllll}\text { Calc. Mass Obsrv. Mass } & \pm \text { da } \pm \text { ppm } & \text { Start } & \text { End Sequence } & \text { lon } & \text { C. I. \% Modification } & \text { Plate [\#] Name } & \text { Gel Idx/Pos [4700 } & \text { iTRAQ } & \text { iTRAQ }\end{array}$

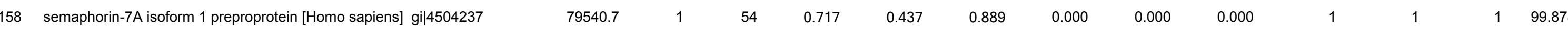
Protein Group

semaphorin L [Mus musculus] $\quad$ gil3523117 $\quad 46843.7$

semaphorin-7A precursor [Mus musculus] $\quad$ gil6755466 $\quad 79376.5$

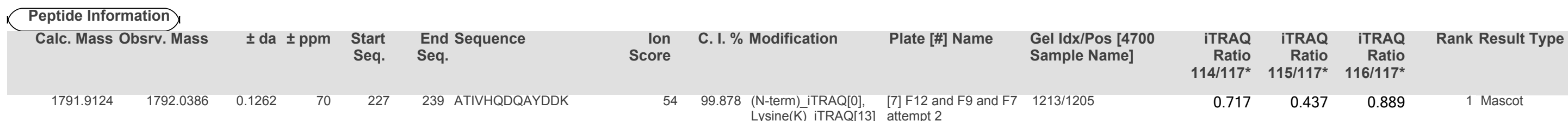

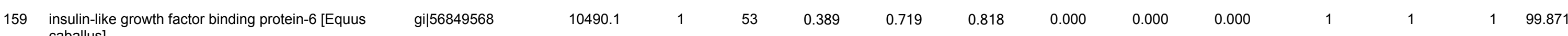
Peptide Information

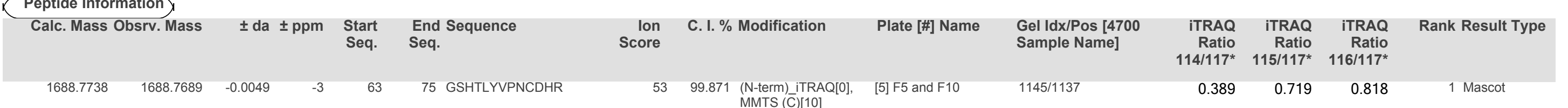

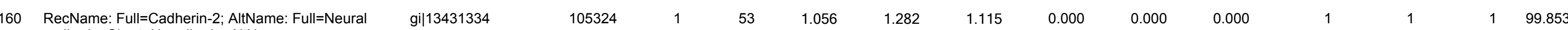
cadherin; Short=N-cadherin; AltName;
CD_antigen=CD32

99.853
Protein Group
Cadherin 2, type 1, N-cadherin (neuronal) [Homo
gi|22209070
105700.1
N-cadherin [Homo sapiens]
gi|253483 $\quad 105664.1$
RecName: Full=Cadherin-2; AltName: Full=Neural
gil115421
102583.6 
neural cadherin precursor [Mus musculus]

\section{Peptide Information}

\begin{tabular}{|c|c|c|c|c|c|c|c|c|c|c|c|}
\hline Calc. Mass Obsrv. Mass & \pm da $\pm p p m$ & $\begin{array}{l}\text { Start } \\
\text { Seq. }\end{array}$ & $\begin{array}{l}\text { End Sequence } \\
\text { Seq. }\end{array}$ & $\begin{array}{l}\text { Ion } \\
\text { Score }\end{array}$ & C. I. \% Modification & Plate [\#] Name & $\begin{array}{l}\text { Gel Id dx/Pos [4700 } \\
\text { Sample Name] }\end{array}$ & $\begin{array}{r}\text { iTRAQ } \\
\text { Ratio } \\
114 / 117^{*}\end{array}$ & $\begin{array}{r}\text { iTRAQ } \\
\text { Ratio } \\
115 / 117^{*}\end{array}$ & $\begin{array}{r}\text { iTRAQ } \\
\text { Ratio } \\
116 / 117^{*}\end{array}$ & Rank Result Type \\
\hline 2045.1973 & 0.0683 & 102 & 114 FLIYAQDKETQEK & 53 & 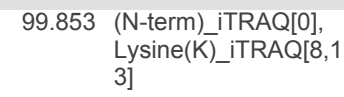 & {$[3] \mp 14,15,16$ and $F 6$} & $852 / 844$ & 1.056 & 1.282 & 1.115 & 1 Mascot \\
\hline
\end{tabular}

161 RecName: Full=Complement C2; AltName: Full=C3/C5 gil48427894 convertase; Contains: RecName: Full=Complement
$\mathrm{C} 2 \mathrm{~b}$

897078

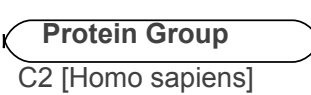

C2 [Homo sapiens]

gil15277207

68378.1

DKFZP469A1324 protein [Pongo abelii]

gi|207079949

88115.9

RecName: Full=Complement C2; AltName: Full=C3/C5 gi|62286486

convertase; Contains: RecName: Full=Complement

RecName: Full=Complement C2; AltName: Full=C3/C5 gi|38257345

convertase; Contains: RecName: Full=Complement

complement C2 [Pongo abelii]

gil197098872

complement C2 [Sus scrofa]

gi|38455778 $\quad 879993$

complement C2 isoform 1 preproprotein [Homo sapiens] gi|14550407

89649.8

$\begin{array}{lll}\text { hypothetical protein [Homo sapiens] } & \text { gi|31873561 } & 44416.4\end{array}$

unnamed protein product [Homo sapiens] $\quad$ gi|34628 $\quad 89615.8$

\begin{tabular}{|c|c|c|c|c|c|c|c|c|c|c|c|}
\hline Calc. Mass Obsrv. Mass & $\pm \mathrm{da} \pm \mathrm{ppm}$ & $\begin{array}{l}\text { Start } \\
\text { Seq. }\end{array}$ & $\begin{array}{l}\text { End Sequence } \\
\text { Seq. }\end{array}$ & $\begin{array}{l}\text { lon } \\
\text { Score }\end{array}$ & C. I. \% Modification & Plate [\#] Name & $\begin{array}{l}\text { Gel Idd/Pos [4700 } \\
\text { Sample Name] }\end{array}$ & $\begin{array}{r}\text { iTRAQ } \\
\text { Ratio } \\
114 / 117^{*}\end{array}$ & $\begin{array}{r}\text { iTRAQ } \\
\text { Ratio } \\
115 / 117^{*}\end{array}$ & $\begin{array}{r}\text { iTRAQ } \\
\text { Ratio } \\
116 / 117^{*}\end{array}$ & Rank Result Type \\
\hline 1638.9542 & -0.0525 & 478 & 487 TPWHVTIKPK & 52 & 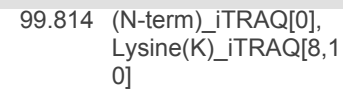 & [4] F11 and F3 & $2121 / 204$ & 0.436 & 0.857 & 0.844 & 1 Mascot \\
\hline
\end{tabular}




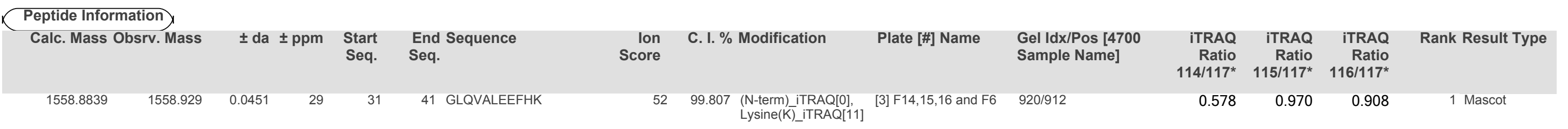

\begin{tabular}{l}
$163 \begin{array}{l}\text { Chain A, Crystal Structure Analysis Of The Bovine Npc2 gi|28373999 } \\
\text { (Niemann-Pick C2) Protein }\end{array}$ \\
\hline
\end{tabular}

Protein Group

\begin{tabular}{|c|c|c|c|c|c|c|c|c|c|c|c|}
\hline Calc. Mass Obsrv. Mass & \pm da \pm ppm & $\begin{array}{l}\text { Start } \\
\text { Seq. }\end{array}$ & $\begin{array}{l}\text { End Sequence } \\
\text { Seq. }\end{array}$ & $\begin{array}{l}\text { lon } \\
\text { Score }\end{array}$ & C. I. \% Modification & Plate [\#] Name & $\begin{array}{l}\text { Gel Idx/Pos [4700 } \\
\text { Sample Name] }\end{array}$ & $\begin{array}{r}\text { iTRAQ } \\
\text { Ratio } \\
114 / 1117^{*}\end{array}$ & $\begin{array}{r}\text { iTRAQ } \\
\text { Ratio } \\
115 / 117^{*}\end{array}$ & $\begin{array}{r}\text { iTRAQ } \\
\text { Ratio } \\
116 / 117^{*}\end{array}$ & Rank Result Type \\
\hline 1576.8706 & 0.0503 & 85 & 93 DKTYNYVNK & 51 & 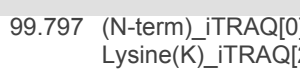 & $\begin{array}{l}{[7] F 12 \text { and } F 9 \text { and } F 7} \\
\text { attempt } 2\end{array}$ & 817/809 & 0.741 & 0.712 & 0.938 & 1 Mascot \\
\hline
\end{tabular}

$\begin{array}{llllllllllllll}164 & \text { Chain A, Crystal Structure Of A Human Igm } & \text { gi|3659940 } & 26076.4 & 1 & 51 & 1.071 & 1.417 & 1.603 & 0.000 & 0.000 & 0.000 & 1 & 1\end{array}$ Rheumatoid Factor Fab In Complex With lis

Autoantigen Igg

Protein Group

Chain A, Crystal Structure Of The C2 Fragment of $\quad$ gil1065199 26396.7

Peptide Information

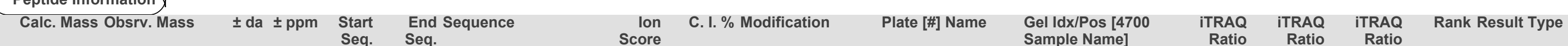

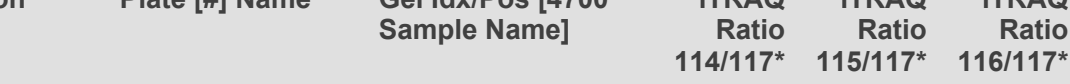

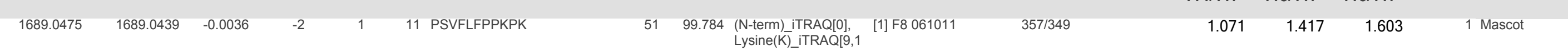

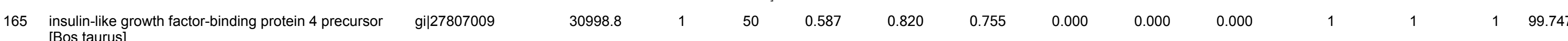
[Bos taurus]

Protein Group

RecName: Full=Insulin-like growth factor-binding protein gi|2497252 28933.7
4: Short=IBP-4; Short=|GF-binding protein

insulin-like growth factor binding protein $4[$ Homo $\quad$ gil54696644 30826.5

sapiens

gi|695254 $\quad 30762.5$

insulin-like
sapiens]

insulin-like growth factor-binding protein 4 [Bos taurus] gil17864013 $\quad 17787.8$

insulin-like growth factor-binding protein 4 precursor $\quad$ gil62243290 30754.5

Peptide Information

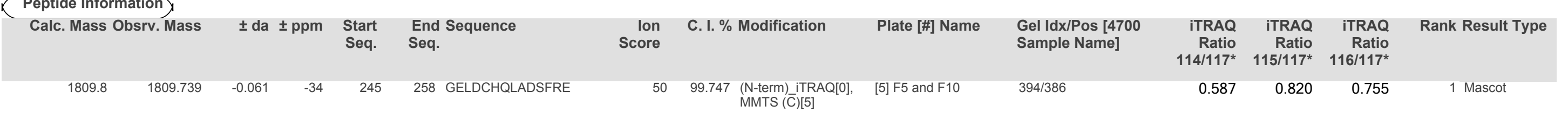

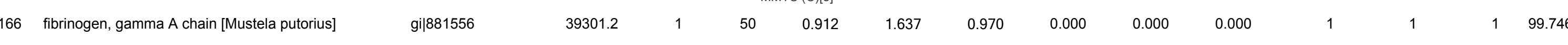

\begin{tabular}{|c|c|c|c|c|c|c|c|c|c|c|c|}
\hline Calc. Mass Obsrv. Mass & \pm da $\pm p p m$ & $\begin{array}{l}\text { Start } \\
\text { Seq. }\end{array}$ & $\begin{array}{l}\text { End Sequence } \\
\text { Seq. }\end{array}$ & $\begin{array}{l}\text { lon } \\
\text { Score }\end{array}$ & C. I. \% Modification & Plate [\#] Name & $\begin{array}{l}\text { Gel Idx/Pos [4700 } \\
\text { Sample Name] }\end{array}$ & $\begin{array}{r}\text { iTRAQ } \\
\text { Ratio } \\
114111^{*}\end{array}$ & $\begin{array}{r}\text { iTRAQ } \\
\text { Ratio } \\
11 / 111^{*}\end{array}$ & $\begin{array}{r}\text { iTRAQ } \\
\text { Ratio } \\
11 / 111^{*}\end{array}$ & Rank Result Type \\
\hline 1785.9381 & 0.0166 & 14 & 25 FLQEIYNSNNQK & 50 & 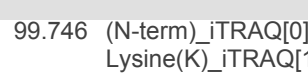 & [2] F4 and F13 & 193/185 & 0.912 & 1.637 & 0.970 & Mascot \\
\hline
\end{tabular}

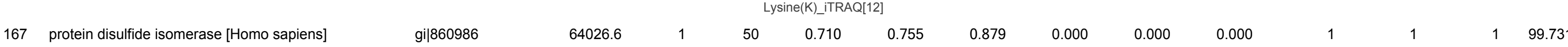




\begin{tabular}{|c|c|c|}
\hline Protein Group & & \\
\hline & gi|1208427 & 64143.7 \\
\hline ER-60 protein [Homo sapiens] & gi|2245365 & 64130.6 \\
\hline $\begin{array}{l}\text { RecName: Full=Protein disulfide-isomerase A3; } \\
\text { AltName: Full=58 kDa glucose-regulated protein; AltNa }\end{array}$ & gil729433 & 64709.1 \\
\hline phospholipase C-alpha [Homo sapiens] & gi|303618 & 64048.7 \\
\hline & gi|21361657 & 64129.7 \\
\hline
\end{tabular}

sapiens]

\section{Peptide Information}

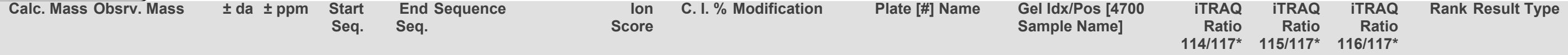

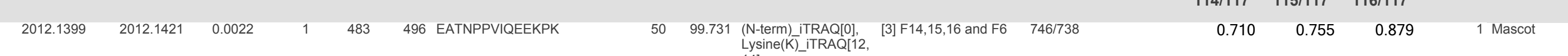

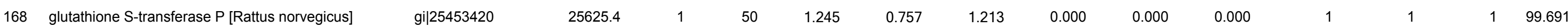

Protein Group

Chain A, Structure Of Porcine Class Pi Glutathione $\quad$ gil1943418 25697

RecName: Full=Glutathione S-transferase P; AltName: gil544445 25683.4
Full=GST P1-1; AltName: Full=GST class-pi

RecName: Full=Glutathione S-transferase P; AltName: $\quad$ gil11170100 25968.8
Full=GST class-pi

RecName: Full=Glutathione S-transferase P; AltName: gil1346208 25943.7

Full=GST class-pi

glutathione S-transferase P [Macaca mulatta] $\quad$ gi|1111185949 $\quad 25480.3$

glutathione S-transferase P [Pongo abelii] $\quad$ gi|197097848 25432.3

glutathione S-transferase Pi [Capra hircus] $\quad$ gi|6013379 25541.3

Peptide Information

\begin{tabular}{|c|c|c|c|c|c|c|c|c|c|c|c|}
\hline Calc. Mass Obsrv. Mass & \pm da \pm ppm & $\begin{array}{l}\text { Start } \\
\text { Seq. }\end{array}$ & $\begin{array}{l}\text { End Sequence } \\
\text { Seq. }\end{array}$ & $\begin{array}{l}\text { Ion } \\
\text { Score }\end{array}$ & C. I. \% Modification & Plate [\#] Name & $\begin{array}{l}\text { Gel IdX/Pos [4700 } \\
\text { Sample Name] }\end{array}$ & $\begin{array}{r}\text { iTRAQ } \\
\text { Ratio } \\
114 / 117^{*}\end{array}$ & $\begin{array}{r}\text { iTRAQ } \\
\text { Ratio } \\
115 / 117^{*}\end{array}$ & $\begin{array}{r}\text { iTRAQ } \\
\text { Ratio } \\
116 / 117^{*}\end{array}$ & Rank Result Type \\
\hline 1889.9398 & 0.0287 & 86 & 101 EAALVDMVNDGVEDLR & 50 & 99.691 (N-term)_iTRAQ[0] & ${ }^{[8] \mathrm{F} 2} \mathrm{C}-\mathrm{C}$ & $483 / 475$ & 1.245 & 0.757 & 1.213 & Mascot \\
\hline
\end{tabular}

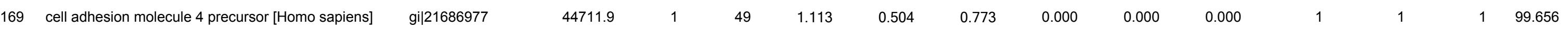

Protein Group

F22162_1 [Homo sapiens] gil3451335 43714.2

cell adhesion molecule 4 precursor [Mus musculus] $\quad$ gi|23346547 $\quad 44649.9$

\section{Peptide Information}

\begin{tabular}{|c|c|c|c|c|c|c|c|c|c|c|c|}
\hline Calc. Mass Obsrv. Mass & $\pm d a \pm p p m$ & $\begin{array}{l}\text { Start } \\
\text { Seq. }\end{array}$ & $\begin{array}{l}\text { End Sequence } \\
\text { Seq. }\end{array}$ & $\begin{array}{l}\text { lon } \\
\text { Score }\end{array}$ & C. I. \% Modification & Plate [\#] Name & $\begin{array}{l}\text { Gel Idx/Pos [4700 } \\
\text { Sample Name] }\end{array}$ & $\begin{array}{r}\text { iTRAQ } \\
\text { Ratio } \\
114 / 1117^{*}\end{array}$ & $\begin{array}{r}\text { iTRAQ } \\
\text { Ratio } \\
115 / 117^{*}\end{array}$ & $\begin{array}{r}\text { iTRAQ } \\
\text { Ratio } \\
116 / 117^{*}\end{array}$ & Rank Result Type \\
\hline
\end{tabular}
170 complement component 3 [Bos taurus]
gi|47271264
8802.6
$\begin{array}{llll}49 & 0.794 & 0.891 & 0.985\end{array}$
Peptide Information
Calc. Mass Obsrv. Mass
\pm da \pm ppm
Start End Sequence

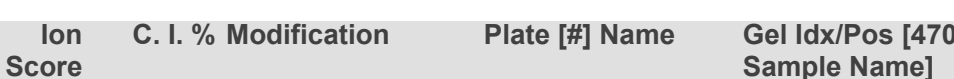

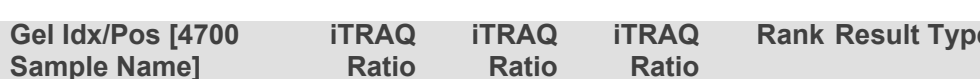

$339 / 331$

$\begin{array}{lll}1.113 & 0.504 & 0.773\end{array}$ 
$2812.231 \quad 2812.2727 \quad 0.0417$

$43 \quad 62$ DTWVELWPEAEECQDEE

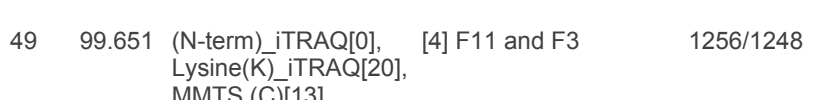

0.794

$0.891 \quad 0.985$

1 Mascot

171 adipsin/complement factor D [Sus scrofa]

gil773265

$49 \quad 1.498$

$\begin{array}{lll}1.270 & 1.087 & 0.000\end{array}$

0.000

0.000

1

199.643

PREDICTED: complement factor D [Sus scrofa]

gi|311248224

29600.9

complement factor D [Sus scrofa]

gi|34329339 $\quad 9673.5$

\section{Peptide Information}

\begin{tabular}{|c|c|c|c|c|c|c|c|c|c|c|c|}
\hline Calc. Mass Obsrv. Mass & \pm da \pm ppm & $\begin{array}{l}\text { Start } \\
\text { Seq. }\end{array}$ & $\begin{array}{l}\text { End Sequence } \\
\text { Seq. }\end{array}$ & $\begin{array}{l}\text { lon } \\
\text { Score }\end{array}$ & C. I. \% Modification & Plate [\#] Name & $\begin{array}{l}\text { Gel Idx/Pos [4700 } \\
\text { Sample Name] }\end{array}$ & $\begin{array}{r}\text { iTRAQ } \\
\text { Ratio } \\
114 / 117^{*}\end{array}$ & $\begin{array}{r}\text { iTRAQ } \\
\text { Ratio } \\
115 / 117^{*}\end{array}$ & $\begin{array}{r}\text { iTRAQ } \\
\text { Ratio } \\
116 / 117^{*}\end{array}$ & Rank Result Type \\
\hline 3372 & 8701 & 99 & 109 LQHLLLPVLDF & 49 & 99.643 (N-term)_iTRAQ & [5] F F and F10 & $472 / 464$ & 1.498 & 1.270 & 1.087 & \\
\hline
\end{tabular}

172 RecName: Full=Pro-neuropeptide $Y$; Contes:

gi|30923117 $\quad 9114.6$

$\begin{array}{lllll}1.401 & 1.536 & 0.000 & 0.000 & 0.000\end{array}$

$1 \quad 1$

$1 \quad 99.642$ RecName: Full=Neuropeptide Y; AltName:
Full=Neuropeptid

Protein Group

[Propertide Y[Homo sapiens]

gi|386984 $\quad 11367.9$

neuropeptide $Y$ precursor [Ovis aries]

gi|3822548 $\quad 10388.5$

pro-neuropeptide Y precursor [Bos taurus]

gi|62460382 11380

pro-neuropeptide $Y$ precursor [Macaca mulatta]

gi|74136189 $\quad 11455.9$

pro-neuropeptide Y precursor [Ovis aries]

gi| 57164209

11366

pro-neuropeptide $Y$ precursor [Rattus norvegicus]

gil6981286 $\quad 11693.9$

pro-neuropeptide $Y$ preproprotein [Homo sapiens]

gi| 4505449

11467

\section{Peptide Information}

\begin{tabular}{|c|c|c|c|c|c|c|c|c|c|c|c|}
\hline Calc. Mass Obsrv. Mass & $\pm \mathrm{da} \pm \mathrm{ppm}$ & $\begin{array}{l}\text { Start } \\
\text { Seq. }\end{array}$ & $\begin{array}{l}\text { End Sequence } \\
\text { Seq. }\end{array}$ & $\begin{array}{l}\text { lon } \\
\text { Score }\end{array}$ & C. I. \% Modification & Plate [\#] Name & $\begin{array}{l}\text { Gel Idx/Pos [4700 } \\
\text { Sample Name] }\end{array}$ & $\begin{array}{r}\text { iTRAQ } \\
\text { Ratio } \\
114 / 117^{*}\end{array}$ & $\begin{array}{r}\text { iTRAQ } \\
\text { Ratio } \\
115 / 117^{*}\end{array}$ & $\begin{array}{r}\text { iTRAQ } \\
\text { Ratio } \\
116 / 117^{*}\end{array}$ & Rank Result Type \\
\hline
\end{tabular}

63835.8

Protein Group
K, And A, Pyruvate Kinase From Rabbit Muscle With Mg, gi|365994actate

Chain A, Recombinant Rabbit Muscle Pyruvate Kinase gil15987970

63769.6

Chain A, S402p Mutant Of Rabbit Muscle Pyruvate gil15987978

63753.6

Kinas

M2 pyruvate kinase [Rattus norvegicus]

gi|206205

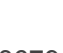

M2-type pyruvate kinase [Homo sapiens]

gil189998

$(38128$

PKM2 protein [Homo sapiens]

gi|34782802

40842.7

Pkm2 protein [Rattus norvegicus]

gi|38181543

58261.7 
RecName: Full=Pyruvate kinase isozymes M1/M2; $\quad$ gi|2851533

cytosolic thyroid hormone-binding protein (EC 2.7.1.40) gi|338827

pyruvate kinase [Homo sapiens]

gi|35505

pyruvate kinase isozymes M1/M2 [Mus musculus]

\section{Peptide Information}

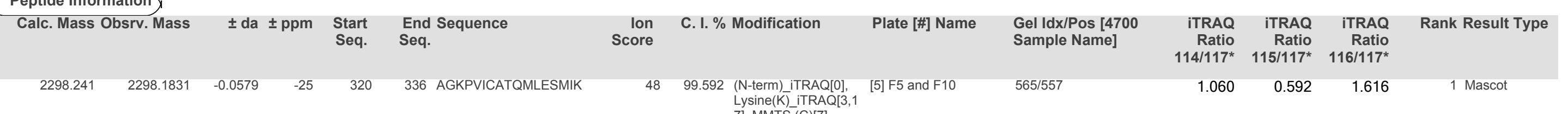

$\begin{array}{llllll}2298.241 & 2298.1831 & -0.0579 & -25 & 320 & 336 \\ \text { AGKPVICATQMLESMIK }\end{array}$ 7], MMTS (C)[7]

\section{4 immunoglobulin $\mathrm{V}$ lambda chain 5.1.11 [Ovis aries] gil26245557}

11278.4

immunoglobulin V lambda chain 5.1.12 [Ovis aries] $\quad$ gi|26245559 $\quad 11303.4$

immunoglobulin V lambda chain 5.1.14 [Ovis aries] $\quad$ gi|26245563 $\quad 11248.3$

immunoglobulin lambda-1a light chain variable region $\quad$ gi|2746683 $\quad 12195.8$
[Ovis aries]

\section{Peptide Information}

\begin{tabular}{|c|c|c|c|c|c|c|c|c|c|c|c|}
\hline Calc. Mass Obsrv. Mass & \pm da \pm ppm & $\begin{array}{l}\text { Start } \\
\text { Seq. }\end{array}$ & $\begin{array}{l}\text { End Sequence } \\
\text { Seq. }\end{array}$ & $\begin{array}{l}\text { lon } \\
\text { Score }\end{array}$ & C. I. \% Modification & Plate [\#] Name & $\begin{array}{l}\text { Gell Idx/Pos [4700 } \\
\text { Sample Name] }\end{array}$ & $\begin{array}{r}\text { iTRAQ } \\
\text { Ratio } \\
114 / 117^{*}\end{array}$ & $\begin{array}{r}\text { iTRAQ } \\
\text { Ratio } \\
115 / 117^{*}\end{array}$ & $\begin{array}{r}\text { iTRAQ } \\
\text { Ratio } \\
116 / 117^{*}\end{array}$ & Rank Result Type \\
\hline
\end{tabular}

$\begin{array}{llllllllllllll}1862.9845 & 1862.8909 & -0.0936 & -50 & 56 & 71 & \text { TVIYGSTNRPSGVPDR } & 48 & 99.588 \text { (N-term) iTRAQ[0] } & \text { [5] F5 and F10 } & 237 / 229 & 1.310 & 0.793 & 1245\end{array}$

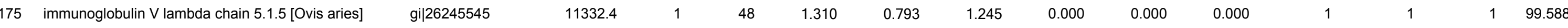
Peptide Information

\begin{tabular}{|c|c|c|c|c|c|c|c|c|c|c|c|}
\hline Calc. Mass Obsrv. Mass & \pm da $\pm p p m$ & $\begin{array}{l}\text { Start } \\
\text { Seq. }\end{array}$ & $\begin{array}{l}\text { End Sequence } \\
\text { Seq. }\end{array}$ & $\begin{array}{l}\text { lon } \\
\text { Score }\end{array}$ & C. I. \% Modification & Plate [\#] Name & $\begin{array}{l}\text { Gel Idx/Pos [4700 } \\
\text { Sample Name] }\end{array}$ & $\begin{array}{r}\text { iTRAQ } \\
\text { Ratio } \\
114 / 117^{*}\end{array}$ & $\begin{array}{r}\text { iTRAQ } \\
\text { Ratio } \\
115 / 117^{*}\end{array}$ & $\begin{array}{r}\text { ITRAQ } \\
\text { Ratio } \\
116 / 117^{*}\end{array}$ & Rank Result Type \\
\hline
\end{tabular}

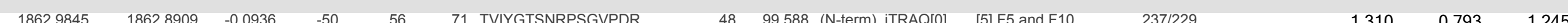

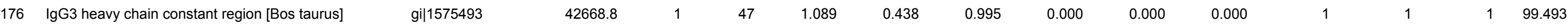
Protein Group

IgG3 heavy chain constant region [Bos taurus] $\quad$ gil1575495 42480.6

\begin{tabular}{|c|c|c|c|c|c|c|c|c|c|c|c|}
\hline Calc. Mass Obsrrv. Mass & \pm da \pm ppm & Start & End Sequence & $\begin{array}{l}\text { Ion } \\
\text { SSore }\end{array}$ & C. I. \% Modification & Plate [\#] Name & Gel Idx/Pos [4700 & iTRAQ & iTRAQ & iTRAQ & Rank Result Type \\
\hline & & & & & & & & $\begin{array}{r}\text { Ratio } \\
114 / 117^{*} \\
1.089\end{array}$ & $\begin{array}{r}\text { Ratio } \\
115 / 117^{*} \\
0.438\end{array}$ & $\begin{array}{r}\text { Ratio } \\
116 / 117^{*} \\
0.995\end{array}$ & \\
\hline
\end{tabular}
177 nidogen-2 [Homo sapiens]
gi|2791962
158019.9
$\begin{array}{llll}47 & 1.253 & 1.218 & 0.705\end{array}$
$0.000 \quad 0.000$
0.000
1
199.488 


\begin{tabular}{lcc}
\hline Protein Group & gil3513368 & 160198 \\
hypactin-2 [Mus musculus] & gi|60219225 & 135257 \\
nidogen-2 [Mus musculus] & gi|23592218 & 159959.2 \\
nidogen-2 precursor [Mus musculus] & gi|84370361 & 159816 \\
osteonidogen [Homo sapiens] & gil1449167 & 158060.9 \\
unnamed protein product [Mus musculus] & gi|26343027 & 146200.1
\end{tabular}

\begin{tabular}{|c|c|c|c|c|c|c|c|c|c|c|c|}
\hline Calc. Mass Obsrv. Mass & $\pm \mathrm{da} \pm \mathrm{ppm}$ & $\begin{array}{l}\text { Start } \\
\text { Seq. }\end{array}$ & $\begin{array}{l}\text { End Sequence } \\
\text { Seq. }\end{array}$ & $\begin{array}{l}\text { Ion } \\
\text { Score }\end{array}$ & C. I. \% Modification & Plate [\#] Name & $\begin{array}{l}\text { Gel Idx/Pos [4700 } \\
\text { Sample Name] }\end{array}$ & $\begin{array}{r}\text { iTRAQ } \\
\text { Ratio } \\
114 / 1117^{*}\end{array}$ & $\begin{array}{r}\text { iTRAQ } \\
\text { Ratio } \\
115 / 1117^{*}\end{array}$ & $\begin{array}{r}\text { iTRAQ } \\
\text { Ratio } \\
116 / 117^{*}\end{array}$ & Rank Result Type \\
\hline 1561.8458 & 0.0202 & 213 & 224 ESYNVQLQLPAR & 47 & 99.488 (N-term)_iTRAQ[0] & [4] F11 and F3 & 1083/1075 & 1.253 & 1.218 & 0.705 & 1 Mascot \\
\hline
\end{tabular}

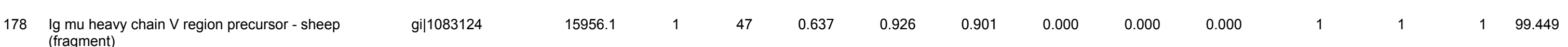

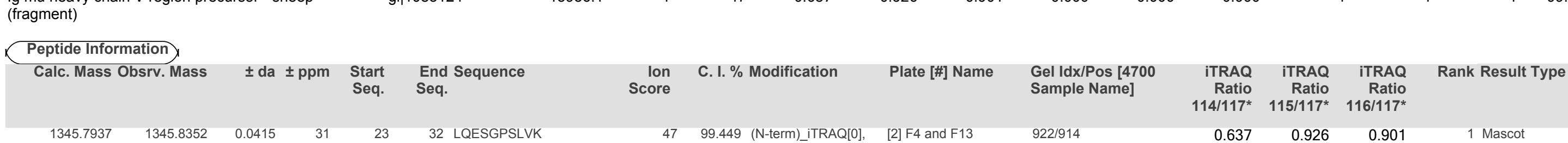

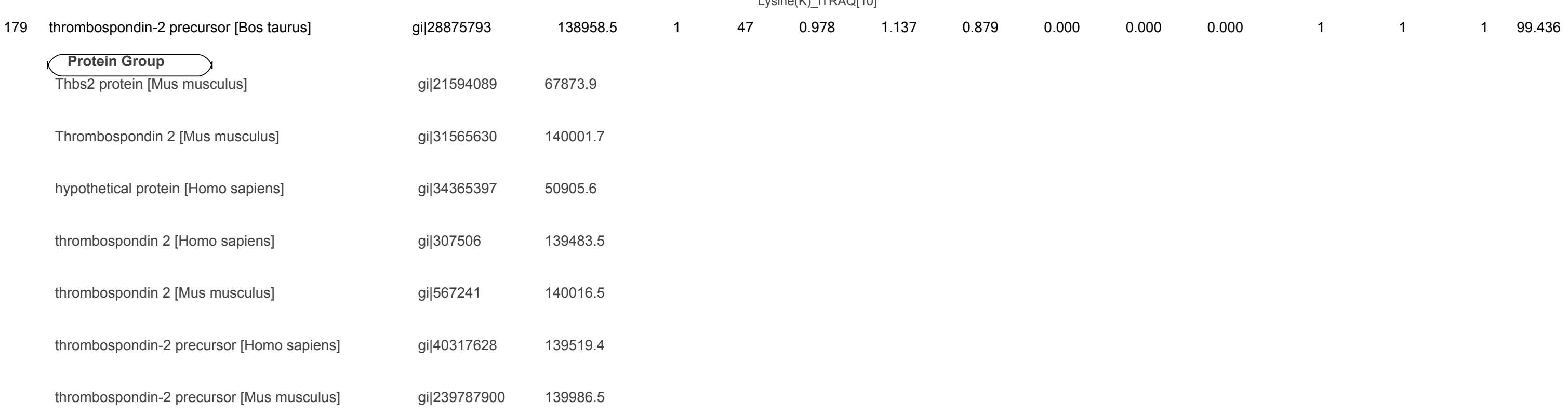


Peptide Information

\begin{tabular}{|c|c|c|c|c|c|c|c|c|c|c|c|}
\hline Calc. Mass Obsrv. Mass & $\pm d a \pm p p m$ & $\begin{array}{l}\text { Start } \\
\text { Seq. }\end{array}$ & $\begin{array}{l}\text { End Sequence } \\
\text { Seq. }\end{array}$ & $\begin{array}{l}\text { Ion } \\
\text { Score }\end{array}$ & C. I. \% Modification & Plate [\#] Name & $\begin{array}{l}\text { Gel Idx/Pos [4700 } \\
\text { Sample Name] }\end{array}$ & $\begin{array}{r}\text { ITRAQ } \\
\text { Ratio } \\
114 / 117^{*}\end{array}$ & $\begin{array}{r}\text { iTRAQ } \\
\text { Ratio } \\
115 / 117^{*}\end{array}$ & $\begin{array}{r}\text { iTRAQ } \\
\text { Ratio } \\
116 / 117^{*}\end{array}$ & Rank Result Type \\
\hline
\end{tabular}

181 SPARC-like protein 1 precursor [Rattus norvegicus] gil6978789

Protein Group

Protein Group

gi|207080142 82764

Hevin-like protein [Homo sapiens]

gil809027 $\quad 82663$

SC1 precursor [Mus musculus]

gi|1854948 $\quad 79504.3$

SPARC-like 1 (hevin) [Homo sapiens]

gi|21707436 $\quad 82645.8$

SPARC-like 1 (hevin) [Rattus norvegicus] $\quad$ gil38197696 77696.4

SPARC-like protein 1 [Pongo abelii]

gi|197101799 82599.9

Sparcl1 protein [Mus musculus]

gil13277723 79444.2

extracellular matrix associated protein [Mus musculus] $\quad$ gi|1498641 79446.2

hevin [Homo sapiens]

gi|758066 $\quad 82631.8$

hypothetical protein [Pongo abelii]

gi|55732071 $\quad 826569$

Peptide Information

\begin{tabular}{|c|c|c|c|c|c|c|c|c|c|c|c|c|}
\hline Calc. Mass Obsrv. Mass & \pm da & $\pm \mathrm{ppm}$ & $\begin{array}{l}\text { Start } \\
\text { Seq. }\end{array}$ & $\begin{array}{l}\text { End Sequence } \\
\text { Seq. }\end{array}$ & $\begin{array}{l}\text { lon } \\
\text { Score }\end{array}$ & C. I. \% Modification & Plate [\#] Name & $\begin{array}{l}\text { Gel Idx/Pos [4700 } \\
\text { Sample Name] }\end{array}$ & $\begin{array}{r}\text { iTRAQ } \\
\text { Ratio } \\
114 / 117^{*}\end{array}$ & $\begin{array}{r}\text { iTRAQ } \\
\text { Ratio } \\
115 / 117^{*}\end{array}$ & $\begin{array}{r}\text { iTRAQ } \\
\text { Ratio } \\
116 / 117^{*}\end{array}$ & Rank Result Type \\
\hline
\end{tabular}

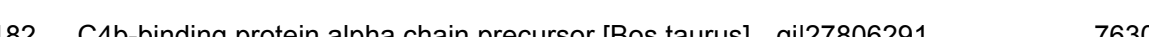

$\begin{array}{lllllllll} & 1 & 46 & 1.140 & 0.584 & 0.981 & 0.000 & 0.000 & 0.000\end{array}$

1

Peptide Information

\begin{tabular}{|c|c|c|c|c|c|c|c|c|c|c|c|}
\hline Calc. Mass Obsrv. Mass & \pm da $\pm p p m$ & $\begin{array}{l}\text { Start } \\
\text { Seq. }\end{array}$ & $\begin{array}{l}\text { End Sequence } \\
\text { Seq. }\end{array}$ & $\begin{array}{l}\text { lon } \\
\text { Score }\end{array}$ & C. I. \% Modification & Plate [\#] Name & $\begin{array}{l}\text { Gel Idx/Pos [4700 } \\
\text { Sample Name] }\end{array}$ & $\begin{array}{r}\text { iTRAQ } \\
\text { Ratio } \\
114 / 117^{*}\end{array}$ & $\begin{array}{r}\text { iTRAQ } \\
\text { Ratio } \\
115 / 117^{*}\end{array}$ & $\begin{array}{r}\text { iTRAQ } \\
\text { Ratio } \\
11 / 117^{*}\end{array}$ & Rank Result Type \\
\hline & -0.0446 & 541 & 556 CEWEYPEGCEQVVTGR & 46 & 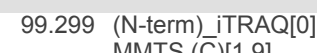 & [8] F2 & $477 / 469$ & 1.140 & 0.584 & 0.981 & lascot \\
\hline
\end{tabular}

183 protein C prepropeptide [Bos taurus]

gi|163487 $\quad 55951$

$46 \quad 0.934 \quad 0.678$

$0.687 \quad 0.000$

0.000

0.000

1

$\begin{array}{lll}1 & 1 & 99.289\end{array}$

Protein Group

RecName: Full=Vitamin K-dependent protein C; $\quad$ gil131065 55937.2

\begin{tabular}{|c|c|c|c|c|c|c|c|c|c|c|c|}
\hline Calc. Mass Obsrv. Mass & $\pm \mathrm{da} \pm \mathrm{ppm}$ & $\begin{array}{l}\text { Start } \\
\text { Seq. }\end{array}$ & $\begin{array}{l}\text { End Sequence } \\
\text { Seq. }\end{array}$ & $\begin{array}{l}\text { Ion } \\
\text { Score }\end{array}$ & C. I. \% Modification & Plate [\#] Name & $\begin{array}{l}\text { Gel Idx/Pos [4700 } \\
\text { Sample Name] }\end{array}$ & $\begin{array}{r}\text { iTRAQ } \\
\text { Ratio } \\
114 / 117^{*}\end{array}$ & $\begin{array}{r}\text { iTRAQ } \\
\text { Ratio } \\
115 / 117^{*}\end{array}$ & $\begin{array}{r}\text { iTRAQ } \\
\text { Ratio } \\
116 / 117^{*}\end{array}$ & Rank Result Type \\
\hline 1595.8832 & 0.0736 & 435 & 444 YLDWIYGHIK & 46 & 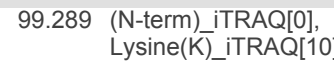 & {$[3] \mathrm{F} 14,15,16$ and $\mathrm{F} 6$} & 1038/1030 & 0.934 & 0.678 & 0.687 & 1 Mascot \\
\hline
\end{tabular}

$\begin{array}{lllllllllllllll}184 & \text { alpha globin chain [Ovis aries] } & \text { gi|1787 } & 16968.1 & 1 & 46 & 0.948 & 1.562 & 1.878 & 0.000 & 0.000 & 0.000 & 1 & 1 & 1\end{array}$ Protein Group 


\section{Peptide Information}

\begin{tabular}{|c|c|c|c|c|c|c|c|c|c|c|c|}
\hline Calc. Mass Obsrv. Mass & $\pm \mathrm{da} \pm \mathrm{ppm}$ & $\begin{array}{l}\text { Start } \\
\text { Seq. }\end{array}$ & $\begin{array}{l}\text { End Sequence } \\
\text { Seq. }\end{array}$ & $\begin{array}{l}\text { Ion } \\
\text { Score }\end{array}$ & C. I. \% Modification & Plate [\#] Name & $\begin{array}{l}\text { Gel Idx/Pos [4700 } \\
\text { Sample Name] }\end{array}$ & $\begin{array}{r}\text { ITRAQ } \\
\text { Ratio } \\
114 / 117^{*}\end{array}$ & $\begin{array}{r}\text { iTRAQ } \\
\text { Ratio } \\
115 / 117^{*}\end{array}$ & $\begin{array}{r}\text { iTRAQ } \\
\text { Ratio } \\
116 / 117^{*}\end{array}$ & Rank Result Type \\
\hline
\end{tabular}

185 galectin 1 [Ovis aries]

gil57164313 16381.

145

$1.461 \quad 1.097$

1.359

$0.000 \quad 0.000$

0.000

1

$\begin{array}{lll}1 & 1 & 99.159\end{array}$

Peptide Information

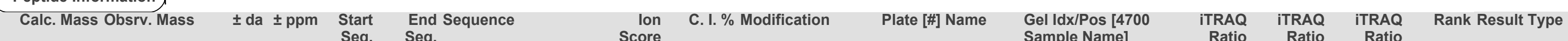

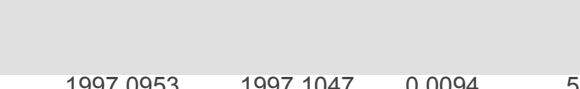

neuroblastoma suppressor of tumorigenicity 1 precursor gi|13928832 20836.7
[Rattus norvegicus]

$\begin{array}{rrr}\text { Ratio } \\ 114 / 117^{*} & 115 \text { Ratio } \\ 1 & 117^{*} & \text { Ratio } \\ 116 / 117^{*}\end{array}$

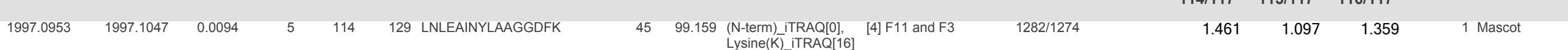

Protein Group
DAN [Mus musculus]

Neuroblastoma, suppression of tumorigenicity 1 [Rattus gi|38197666 20744.7

RecName: Full=Neuroblastoma suppressor of $\quad$ gi|729293 20876.8

tumorigenicity 1; AltName: Full=DAN domain family
member

neuroblastoma suppressor of tumorigenicity 1 isoform 2 gil323276673
precursor [Homo spiens]

21007.9

Peptide Information

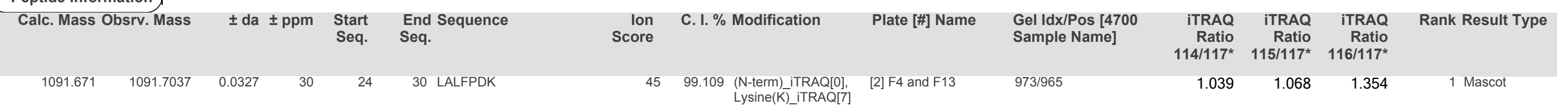

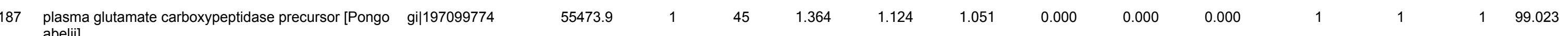

Protein Group

blood plasma glutamate carboxypeptidase precursor $\quad$ gi|4877698 64872

plasma glutamate carboxypeptidase precursor [Homo gi|7706387 55641

$\begin{array}{ll}\text { unnamed protein product [Homo sapiens] } & \text { gi|22761023 }\end{array}$

Peptide Information

\begin{tabular}{|c|c|c|c|c|c|c|c|c|c|c|c|}
\hline Calc. Mass Obsrv. Mass & \pm da \pm ppm & $\begin{array}{l}\text { Start } \\
\text { Seq. }\end{array}$ & $\begin{array}{l}\text { End Sequence } \\
\text { Seq. }\end{array}$ & $\begin{array}{l}\text { lon } \\
\text { Score }\end{array}$ & C. I. \% Modification & Plate [\#] Name & $\begin{array}{l}\text { Gel Idx/Pos [4700 } \\
\text { Sample Name] }\end{array}$ & $\begin{array}{r}\text { iTRAQ } \\
\text { Ratio } \\
114 / 117^{*}\end{array}$ & $\begin{array}{r}\text { iTRAQ } \\
\text { Ratio } \\
115 / 117^{*}\end{array}$ & $\begin{array}{r}\text { iTRAQ } \\
\text { Ratio } \\
116 / 117^{*}\end{array}$ & Rank Result Type \\
\hline & -21 & 67 & 77 LALLVDTVGPR & 45 & 99.023 (N-term) 1 TRAQ[0] & {$[8] \mathrm{F} 2$} & 441/433 & 1.364 & 1.124 & 1.051 & \\
\hline
\end{tabular}

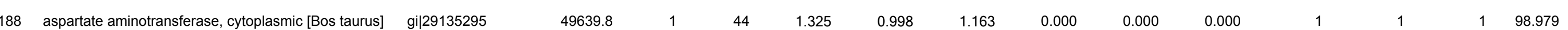
Protein Group

RecName: Full=Aspartate aminotransferase,

cytoplasmic; AltName: Full=Glutamate oxaloacetate

aspartate aminotransferase 1 [Bos taurus]

gi|122065118

49753.7

gil59858077 $\quad 49464.5$ 
Peptide Information

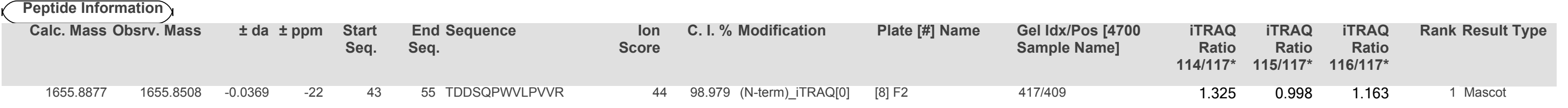

$\begin{array}{lcc}\begin{array}{l}\text { repulsive guidance molecule A isoform 3 [Homo } \\ \text { sapiens] }\end{array} & \text { gi|261878452 } & 53091.5 \\ \begin{array}{l}\text { Protein Group } \\ \text { hypothetical protein [Homo sapiens] }\end{array} & \text { gi|9368530 } & 54438.1 \\ \begin{array}{l}\text { repulsive guidance molecule A isoform 2 [Homo } \\ \text { sapiens] }\end{array} & \text { gi|261878459 } & 51257.5 \\ \begin{array}{l}\text { repulsive guidance molecule A precursor [Mus } \\ \text { musculus] }\end{array} & \text { gi|164663868 } & 53359.8 \\ \begin{array}{l}\text { repulsive guidance molecule A, RgmA protein [Mus } \\ \text { muscullus] }\end{array} & \text { gi|l32400242 } & 51525.8 \\ \begin{array}{l}\text { unnamed protein product [Homo sapiens] } \\ \text { uil22760663 }\end{array} & 53063.5 \\ \text { unnamed protein product [Mus musculus] } & \text { gi|26349781 } & 40992.8\end{array}$

\section{Peptide Information}

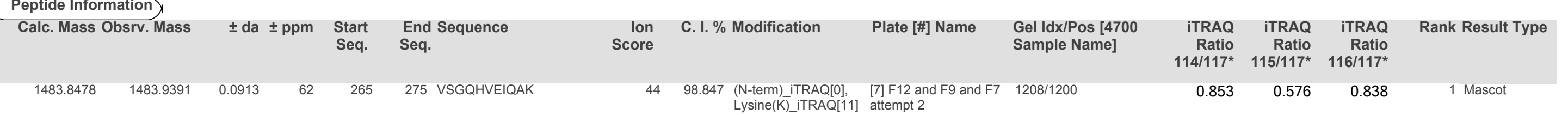

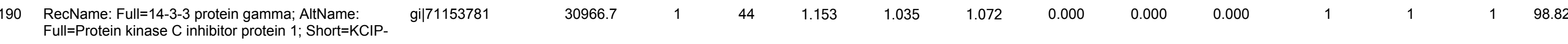

Protein Group

\begin{tabular}{lll}
$14-3-3$ gamma protein [Homo sapiens] & gil5726310 & 30944.5 \\
$14-3-3$ protein gamma [Mus musculus] & gil3065929 & 31076.8 \\
\hline & &
\end{tabular}

14-3-3 protein gamma [Rattus norvegicus] $\quad$ gi|9507245 31016.7

\begin{tabular}{|c|c|c|c|c|c|c|c|c|c|c|c|}
\hline Calc. Mass Obsrv. Mass & \pm da \pm ppm & $\begin{array}{l}\text { Start } \\
\text { Seq. }\end{array}$ & $\begin{array}{l}\text { End Sequence } \\
\text { Seq. }\end{array}$ & $\begin{array}{l}\text { lon } \\
\text { Score }\end{array}$ & C. I. \% Modification & Plate [\#] Name & $\begin{array}{l}\text { Gel Idx/Pos [4700 } \\
\text { Sample Name] }\end{array}$ & $\begin{array}{r}\text { iTRAQ } \\
\text { Ratio } \\
114 / 117^{*}\end{array}$ & $\begin{array}{r}\text { iTRAQ } \\
\text { Ratio } \\
115 / 117^{*}\end{array}$ & $\begin{array}{r}\text { iTRAQ } \\
\text { Ratio } \\
116 / 117^{*}\end{array}$ & Rank Result Type \\
\hline 1787.8372 & -0.0523 & 29 & 42 NVTELNEPLSNEER & 44 & 98.82 (N-term)_iTRAQ[0] & [8] F2 & $277 / 269$ & 1.153 & 1.035 & 1.072 & 1 Mascot \\
\hline
\end{tabular}

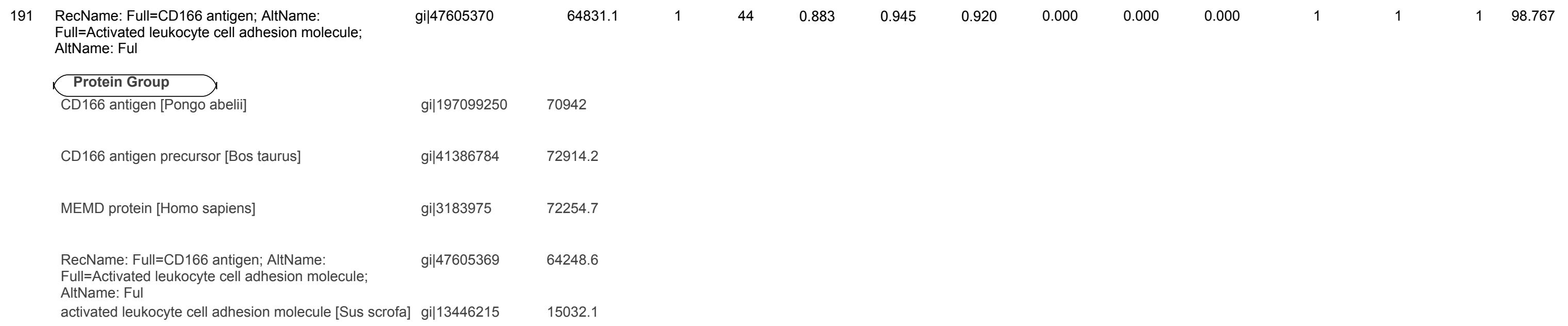


Peptide Information

\begin{tabular}{|c|c|c|c|c|c|c|c|c|c|c|c|}
\hline Calc. Mass Obsrv. Mass & $\pm d a \pm p p m$ & $\begin{array}{l}\text { Start } \\
\text { Seq. }\end{array}$ & $\begin{array}{l}\text { End Sequence } \\
\text { Seq. }\end{array}$ & $\begin{array}{l}\text { lon } \\
\text { Score }\end{array}$ & C. I. \% Modification & Plate [\#] Name & $\begin{array}{l}\text { Gel Idx/Pos [4700 } \\
\text { Sample Name] }\end{array}$ & $\begin{array}{r}\text { iTRAQ } \\
\text { Ratio } \\
114 / 117^{*}\end{array}$ & $\begin{array}{r}\text { iTRAQ } \\
\text { Ratio } \\
115 / 117^{*}\end{array}$ & $\begin{array}{r}\text { iTRAQ } \\
\text { Ratio } \\
116 / 117^{*}\end{array}$ & Rank Result Type \\
\hline 1544.8738 & -0.0646 & 71 & 80 QPSKPEIVSK & 44 & 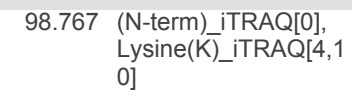 & [1] F8 061011 & 186/178 & 0.883 & 0.945 & 0.920 & 1 Mascot \\
\hline
\end{tabular}

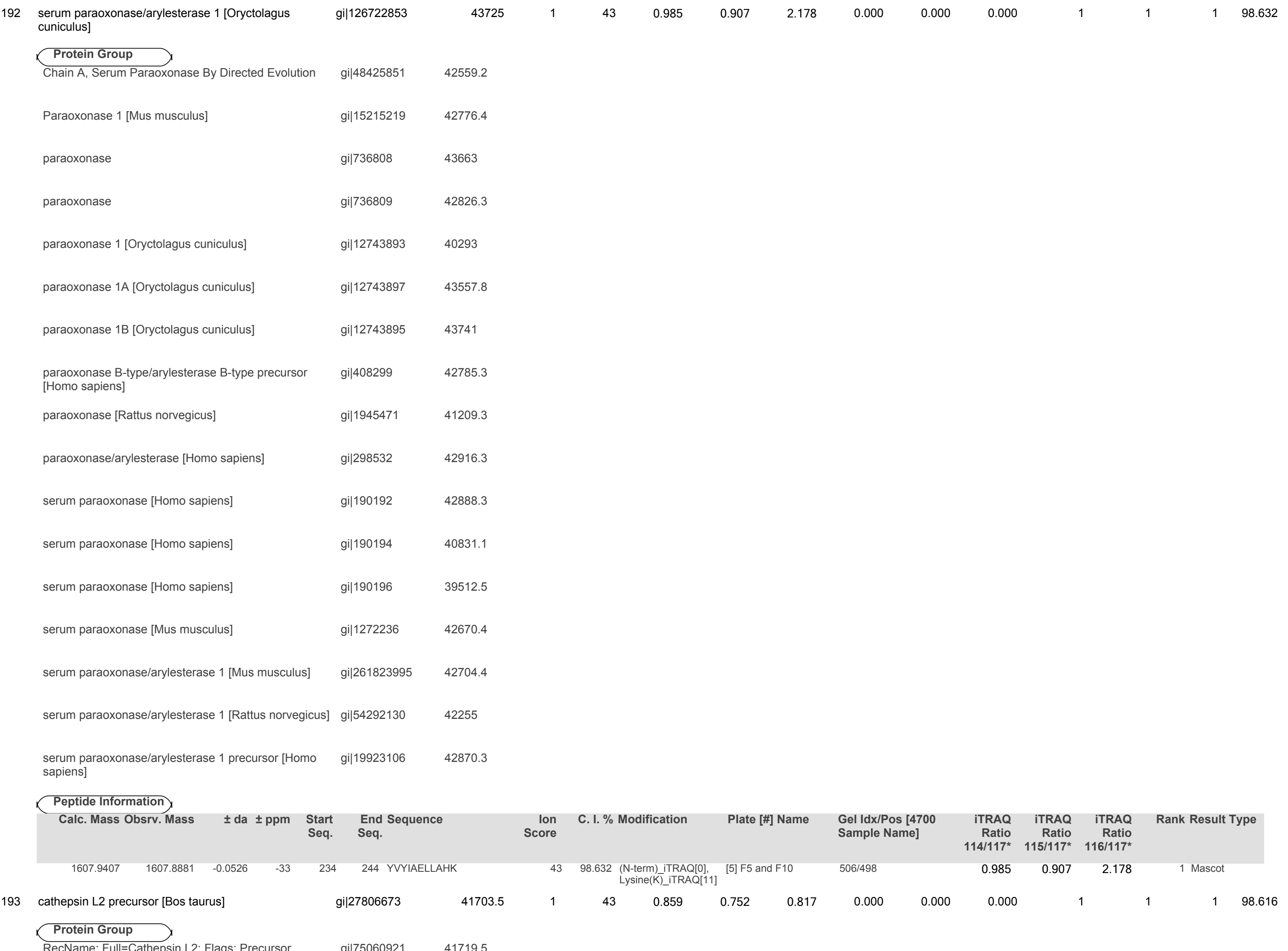




\begin{tabular}{|c|c|c|c|c|c|c|c|c|c|c|c|}
\hline Calc. Mass Obsrv. Mass & $\pm d a \pm p p m$ & $\begin{array}{l}\text { Start } \\
\text { Seq. }\end{array}$ & $\begin{array}{l}\text { End Sequence } \\
\text { Seq. }\end{array}$ & $\begin{array}{l}\text { lon } \\
\text { Score }\end{array}$ & C. I. \% Modification & Plate [\#] Name & $\begin{array}{l}\text { Gel Idx/Pos [4700 } \\
\text { Sample Name] }\end{array}$ & $\begin{array}{r}\text { iTRAQ } \\
\text { Ratio } \\
114 / 117^{*}\end{array}$ & $\begin{array}{r}\text { iTRAQ } \\
\text { Ratio } \\
115 / 117^{*}\end{array}$ & $\begin{array}{r}\text { iTRAQ } \\
\text { Ratio } \\
116 / 117^{*}\end{array}$ & Rank Result Type \\
\hline 1948.0419 & 0.0343 & 21 & 33 LDPNLDAHWHQWK & 43 & 98.616 (N-term)_iTRAQ $[0]$ & [5] F5 and F10 & 1239/1231 & 0.859 & 0.752 & 0.817 & Mascot \\
\hline
\end{tabular}

194 unnamed protein product [Homo sapiens]

\begin{tabular}{lr} 
gi|7022277 & 46412.3 \\
gi|9368808 & 73657.1 \\
gi|9368807 & 74031.2 \\
\hline gi|21706428 & 50809.6 \\
\hline gi|19171211 & 72854.8 \\
\hline gi|l55957679 & 58643.4 \\
\hline gi|47777317 & 74757.5
\end{tabular}

Protein Group

ASPIC [Homo sapiens]

CRTAC1 protein [Homo sapiens]

CRTAC1-B protein [Homo sapiens]

cartilage acidic protein 1 [Homo sapiens]

74757.5

cartilage

Peptide Information

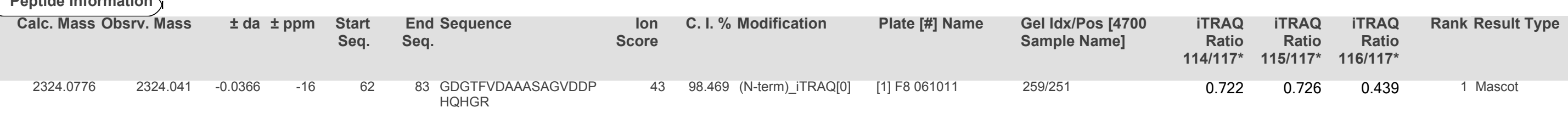

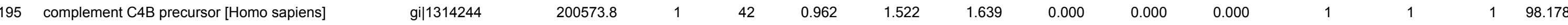
Peptide Information

\begin{tabular}{|c|c|c|c|c|c|c|c|c|c|c|c|}
\hline Calc. Mass Obsrv. Mass & \pm da $\pm p p m$ & $\begin{array}{l}\text { Start } \\
\text { Seq. }\end{array}$ & $\begin{array}{l}\text { End Sequence } \\
\text { Seq. }\end{array}$ & $\begin{array}{l}\text { Ion } \\
\text { Score }\end{array}$ & C. I. \% Modification & Plate [\#] Name & $\begin{array}{l}\text { Gel Idx/Pos [4700 } \\
\text { Sample Name] }\end{array}$ & $\begin{array}{r}\text { iTRAQ } \\
\text { Ratio } \\
114 / 117^{*}\end{array}$ & $\begin{array}{r}\text { iTRAQ } \\
\text { Ratio } \\
115 / 117^{*}\end{array}$ & $\begin{array}{r}\text { iTRAQ } \\
\text { Ratio } \\
116 / 117^{*}\end{array}$ & Rank Result Type \\
\hline 1108.6613 & -0.0512 & 160 & 166 VFXLDQK & 43 & $98.61 \begin{array}{l}\text { (N-term)_iTRAQ[0], } \\
\text { Lysine(K)_iTRAQ[7] }\end{array}$ & [5] F5 and F10 & $283 / 275$ & 0.962 & 1.522 & 1.639 & 1 Mascot \\
\hline
\end{tabular}

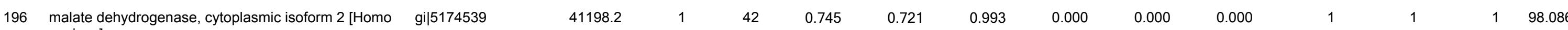

Protein Group

Malate dehydrogenase 1, NAD (soluble) [Rattus $\quad$ gi|37590235 41066.2

cytosolic malate dehydrogenase [Mus musculus] $\quad$ gi|387129 $\quad 41059.2$

malate dehydrogenase, cytoplasmic [Mus musculus] $\quad$ gi|254540027 $\quad 41093.2$

malate dehydrogenase, cytoplasmic [Rattus norvegicus] gil15100179 $\quad 41065.2$

unnamed protein product [Mus musculus] $\quad$ gi|12837652 41050.2

\section{Peptide Information}

\begin{tabular}{|c|c|c|c|c|c|c|c|c|c|c|c|}
\hline Calc. Mass Obsrv. Mass & \pm da $\pm \mathrm{ppm}$ & $\begin{array}{l}\text { Start } \\
\text { Seq. }\end{array}$ & $\begin{array}{l}\text { End Sequence } \\
\text { Seq. }\end{array}$ & $\begin{array}{l}\text { lon } \\
\text { Score }\end{array}$ & C. I. \% Modification & Plate [\#] Name & $\begin{array}{l}\text { Gel Idx/Pos [4700 } \\
\text { Sample Name] }\end{array}$ & $\begin{array}{r}\text { iTRAQ } \\
\text { Ratio } \\
114 / 117^{*}\end{array}$ & $\begin{array}{r}\text { iTRAQ } \\
\text { Ratio } \\
115 / 117^{*}\end{array}$ & $\begin{array}{r}\text { iTRAQ } \\
\text { Ratio } \\
116 / 117^{*}\end{array}$ & Rank Result Type \\
\hline
\end{tabular}




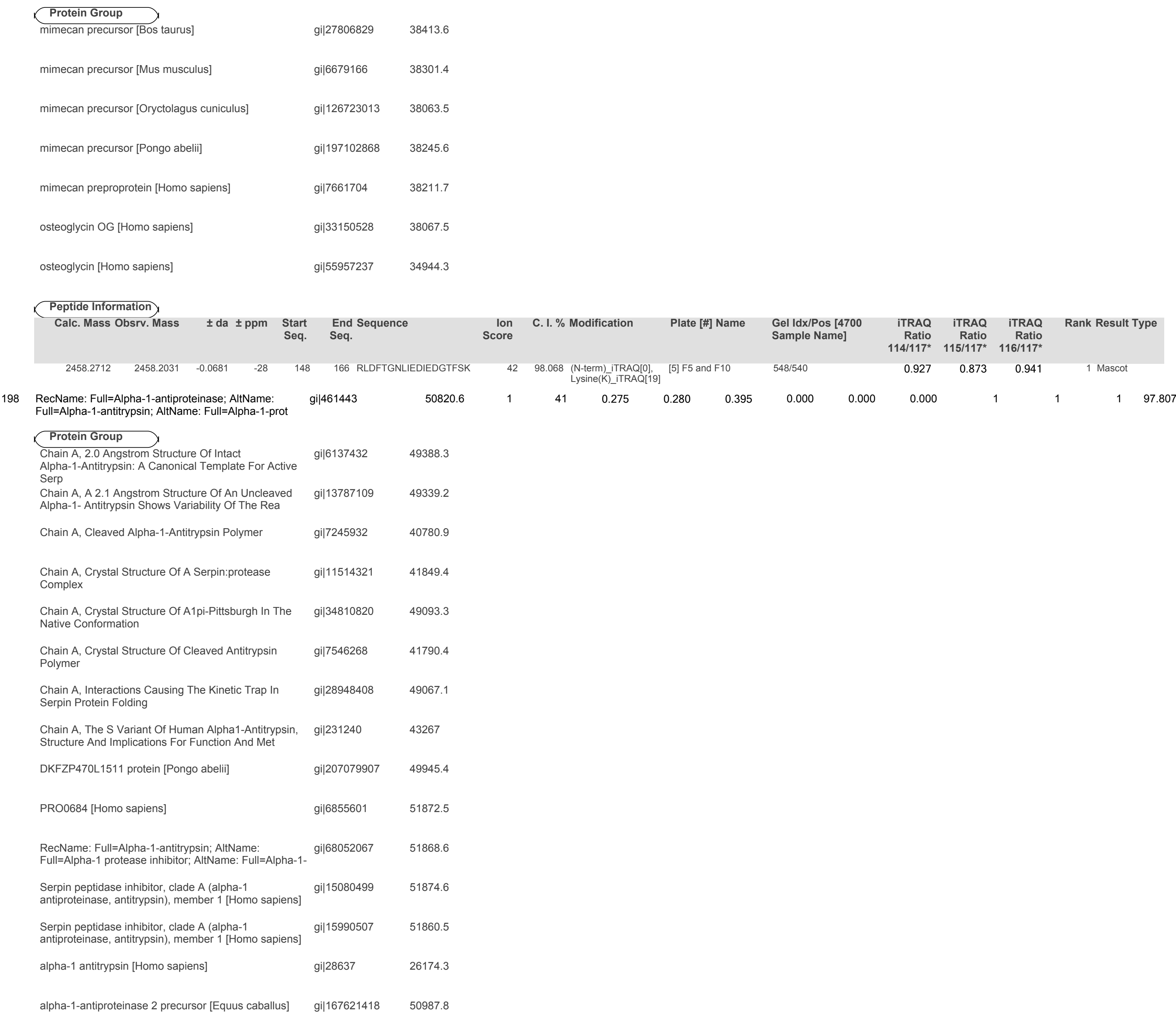




$\begin{array}{llc}\text { alpha-1-antitrypsin [Homo sapiens] } & \text { gi|177827 } & 51797.5 \\ \text { alpha-1-antitrypsin [Homo sapiens] } & \text { gi|177831 } & 51858.6 \\ \text { alpha-1-antitrypsin [Pongo abelii] } & \text { gi|197098210 } & 51944.6 \\ \text { alpha-1-antitrypsin precursor [Homo sapiens] } & \text { gi|50363217 } & 51888.6 \\ \text { alpha-1-antitrypsin precursor [Homo sapiens] } & \text { gi|177836 } & 52003.7 \\ \text { antitrypsin alpha1 mutant } & \text { gi|224224 } & 51883.5 \\ \text { inhibitor,alpha1 protease } & \text { gi|223433 } & 49495.5 \\ \text { unnamed protein product [Homo sapiens] } & \text { gi|28193184 } & 38592.1 \\ \text { unnamed protein product [Homo sapiens] } & \text { gi|28207863 } & 45595\end{array}$

\section{Peptide Information}

\begin{tabular}{|c|c|c|c|c|c|c|c|c|c|c|c|}
\hline Calc. Mass Obsrv. Mass & \pm da $\pm p p m$ & $\begin{array}{l}\text { Start } \\
\text { Seq. }\end{array}$ & $\begin{array}{l}\text { End Sequence } \\
\text { Seq. }\end{array}$ & $\begin{array}{l}\text { lon } \\
\text { Score }\end{array}$ & C. I. \% Modification & Plate [\#] Name & $\begin{array}{l}\text { Gel Idx/Pos [4700 } \\
\text { Sample Name] }\end{array}$ & $\begin{array}{r}\text { iTRAQ } \\
\text { Ratio } \\
114 / 117^{*}\end{array}$ & $\begin{array}{r}\text { iTRAQ } \\
\text { Ratio } \\
115 / 117^{*}\end{array}$ & $\begin{array}{r}\text { iTRAQ } \\
\text { Ratio } \\
116 / 117^{*}\end{array}$ & Rank Result Type \\
\hline 1296.7046 & 0.0681 & 173 & 180 QINDYVEK & 41 & $\begin{array}{c}97.807 \begin{array}{l}\text { (N-term) iTRAQ[0], } \\
\text { Lysine(K) iTRAQ[8] }\end{array}\end{array}$ & $\begin{array}{l}{[7] \mathrm{F} 12 \text { and } \mathrm{F9} \text { and } \mathrm{F7}} \\
\text { attempt } 2\end{array}$ & $823 / 815$ & 0.275 & 0.280 & 0.395 & 1 Mascot \\
\hline
\end{tabular}

$199 \begin{aligned} & \text { RecName: Full=Complement component C6; Flags: } \\ & \text { Precursor }\end{aligned}$ Precursor

\begin{tabular}{|c|c|c|c|c|c|c|c|c|c|c|c|}
\hline Calc. Mass Obsrv. Mass & \pm da $\pm p p m$ & $\begin{array}{l}\text { Start } \\
\text { Seq. }\end{array}$ & $\begin{array}{l}\text { End Sequence } \\
\text { Seq. }\end{array}$ & $\begin{array}{l}\text { lon } \\
\text { Score }\end{array}$ & C. I. \% Modification & Plate [\#] Name & $\begin{array}{l}\text { Gel Idx/Pos [4700 } \\
\text { Sample Name] }\end{array}$ & $\begin{array}{r}\text { iTRAQ } \\
\text { Ratio } \\
114 / 117^{*}\end{array}$ & $\begin{array}{r}\text { iTRAQ } \\
\text { Ratio } \\
115 / 117^{*}\end{array}$ & $\begin{array}{r}\text { iTRAQ } \\
\text { Ratio } \\
116 / 117^{*}\end{array}$ & Rank Result Type \\
\hline $1215.662 \quad 1215.5988$ & -0.0632 & 510 & 517 AFQEYAAK & 41 & $\begin{array}{l}97.772 \begin{array}{l}\text { (N-term) iTRAQ[0], } \\
\text { Lysine(K)_iTRAQ[8] }\end{array}\end{array}$ & [5] F5 and F10 & 232/224 & 0.762 & 1.200 & 0.983 & 1 Mascot \\
\hline
\end{tabular}
200 Ig lambda chain C region - horse (fragment)
gi|346446
13899
$41 \quad 1.206$
$\begin{array}{lll}1.171 & 1.302 & 0.000\end{array}$
$0.000 \quad 0.000$
1
$1 \quad 97.573$

Peptide Information

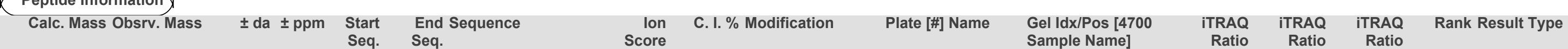

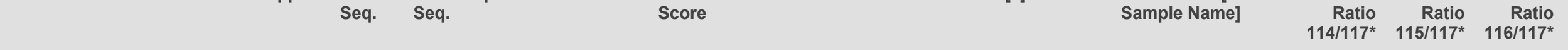

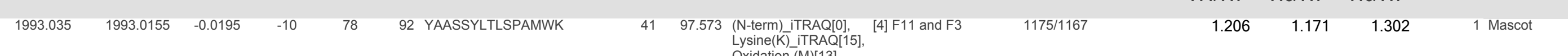

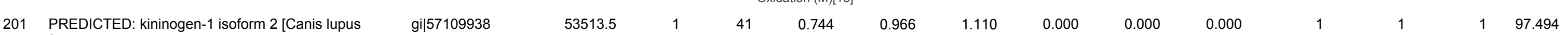

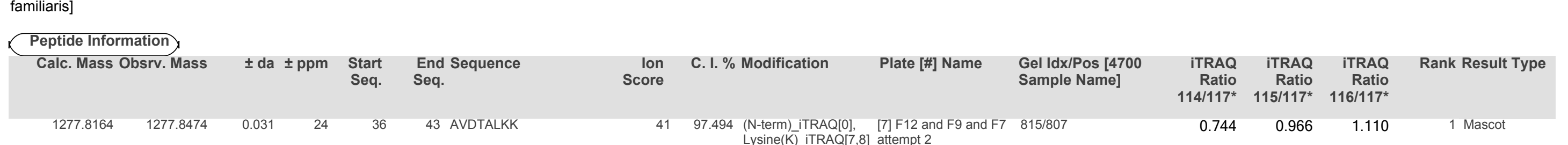

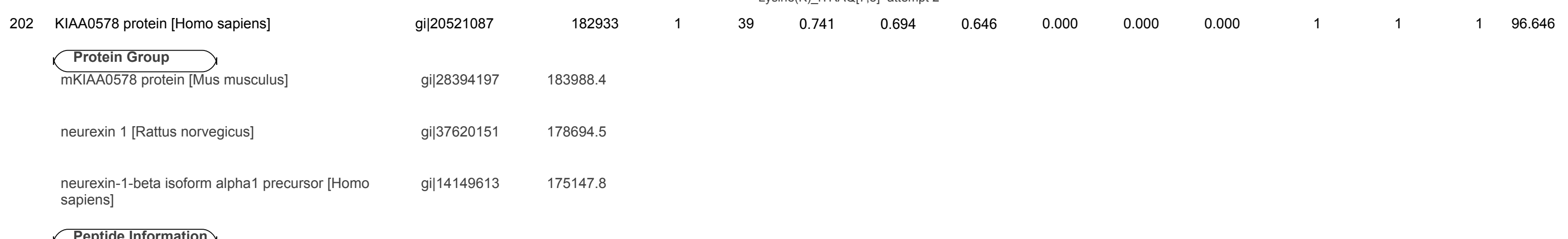

Peptide Information 


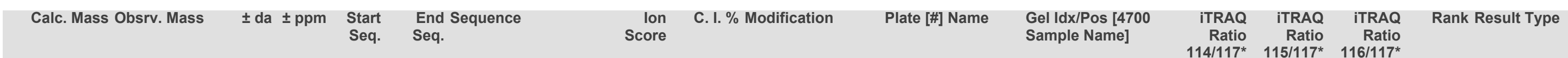

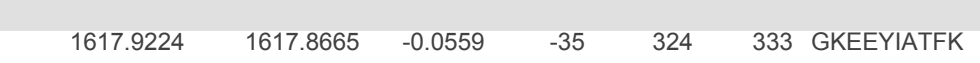

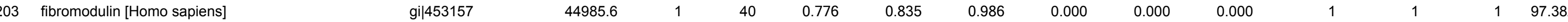

$\begin{array}{lcc}\begin{array}{l}\text { Protein Group } \\ \text { Fmod protein [Mus musculus] }\end{array} & \text { gi|30851675 } & 48402.3 \\ \begin{array}{l}\text { RecName: Full=Fibromodulin; Short=FM; AltName: } \\ \text { Full=Colliagen-binding 59 kDa protein; AltName: Full= }\end{array} & \text { gi|21542068 } & 17122.9 \\ \begin{array}{l}\text { RecName: Full=Fibromodulin; Short=FM; AltName: } \\ \text { Full=Keratan sulfate proteoglycan lumican; Short=KSP }\end{array} & \text { gi|21542112 } & 17252 \\ \text { fibromodulin [Bos taurus] } & \text { gi|27806623 } & 45101.6 \\ \text { fibromodulin [Homo sapiens] } & \text { gi|297091 } & 44936.4 \\ \text { fibromodulin precursor [Homo sapiens] } & \text { gi|71040111 } & 45012.6 \\ \text { fibromodulin precursor [Mus musculus] } & \text { gi|10946680 } & 44790.4 \\ \text { fibromodulin precursor [Rattus norvegicus] } & \text { gi|18104933 } & 44954.5 \\ \text { unnamed protein product [Mus musculus] } & \text { gi|26348627 } & 44933.6\end{array}$

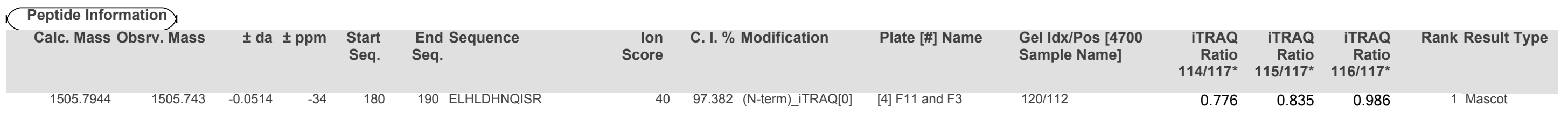

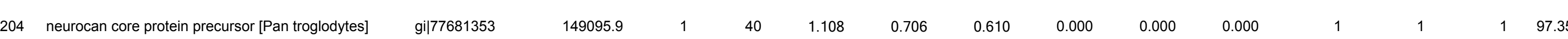
Protein Group

chondroitin sulfate proteoglycan 3 [Saimiri boliviensis] $\quad$ gil56122258 $\quad 134967$

neurocan [Homo sapiens] $\quad$ gi|2739089 $\quad 149057.8$

\begin{tabular}{|c|c|c|c|c|c|c|c|c|c|c|c|}
\hline Calc. Mass Obsrv. Mass & $\pm \mathrm{da} \pm \mathrm{ppm}$ & $\begin{array}{l}\text { Start } \\
\text { Seq. }\end{array}$ & $\begin{array}{l}\text { End Sequence } \\
\text { Seq. }\end{array}$ & $\begin{array}{l}\text { lon } \\
\text { Score }\end{array}$ & C. I. \% Modification & Plate [\#] Name & $\begin{array}{l}\text { Gel Idx/Pos [4700 } \\
\text { Sample Name] }\end{array}$ & $\begin{array}{r}\text { iTRAQ } \\
\text { Ratio } \\
114 / 1117^{*}\end{array}$ & $\begin{array}{r}\text { iTRAQ } \\
\text { Ratio } \\
115 / 117^{*}\end{array}$ & $\begin{array}{r}\text { iTRAQ } \\
\text { Ratio } \\
116 / 1117^{*}\end{array}$ & Rank Result Type \\
\hline 2200.9438 & -0.0735 & 1155 & 1170 DFQWTDNTGLQFENWR & 40 & 97.352 (N-term)_iTRAQ[0] & [8] F2 & $474 / 466$ & 1.108 & 0.706 & 0.610 & 1 Mascot \\
\hline
\end{tabular}

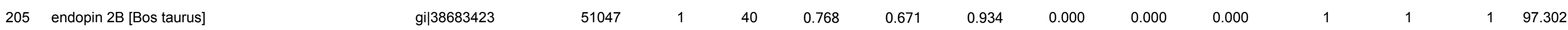
Protein Group

alpha1-antichymotrypsin isoform pHHK12 [Bos taurus] gi|535509 50998.9

\begin{tabular}{|c|c|c|c|c|c|c|c|c|c|c|c|}
\hline Calc. Mass Obsrv. Mass & \pm da \pm ppm & $\begin{array}{l}\text { Start } \\
\text { Seq. }\end{array}$ & $\begin{array}{l}\text { End Sequence } \\
\text { Seq. }\end{array}$ & $\begin{array}{l}\text { Ion } \\
\text { Score }\end{array}$ & C. I. \% Modification & Plate [\#] Name & $\begin{array}{l}\text { Gel Idx/Pos [4700 } \\
\text { Sample Name] }\end{array}$ & $\begin{array}{r}\text { iTRAQ } \\
\text { Ratio } \\
114 / 1117^{*}\end{array}$ & $\begin{array}{r}\text { iTRAQ } \\
\text { Ratio } \\
115 / 1117^{*}\end{array}$ & $\begin{array}{r}\text { iTRAQ } \\
\text { Ratio } \\
116 / 117^{*}\end{array}$ & Rank Result Type \\
\hline 1122.6075 & -0.0732 & 280 & 286 MQDLEAK & 40 & 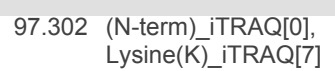 & {$[5] \mathrm{F} 5$ and $\mathrm{F} 10$} & 202/194 & 0.768 & 0.671 & 0.934 & 1 Mascot \\
\hline
\end{tabular}

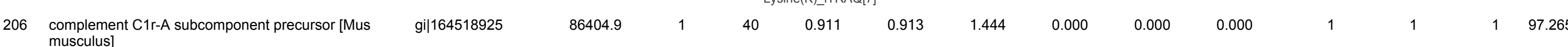




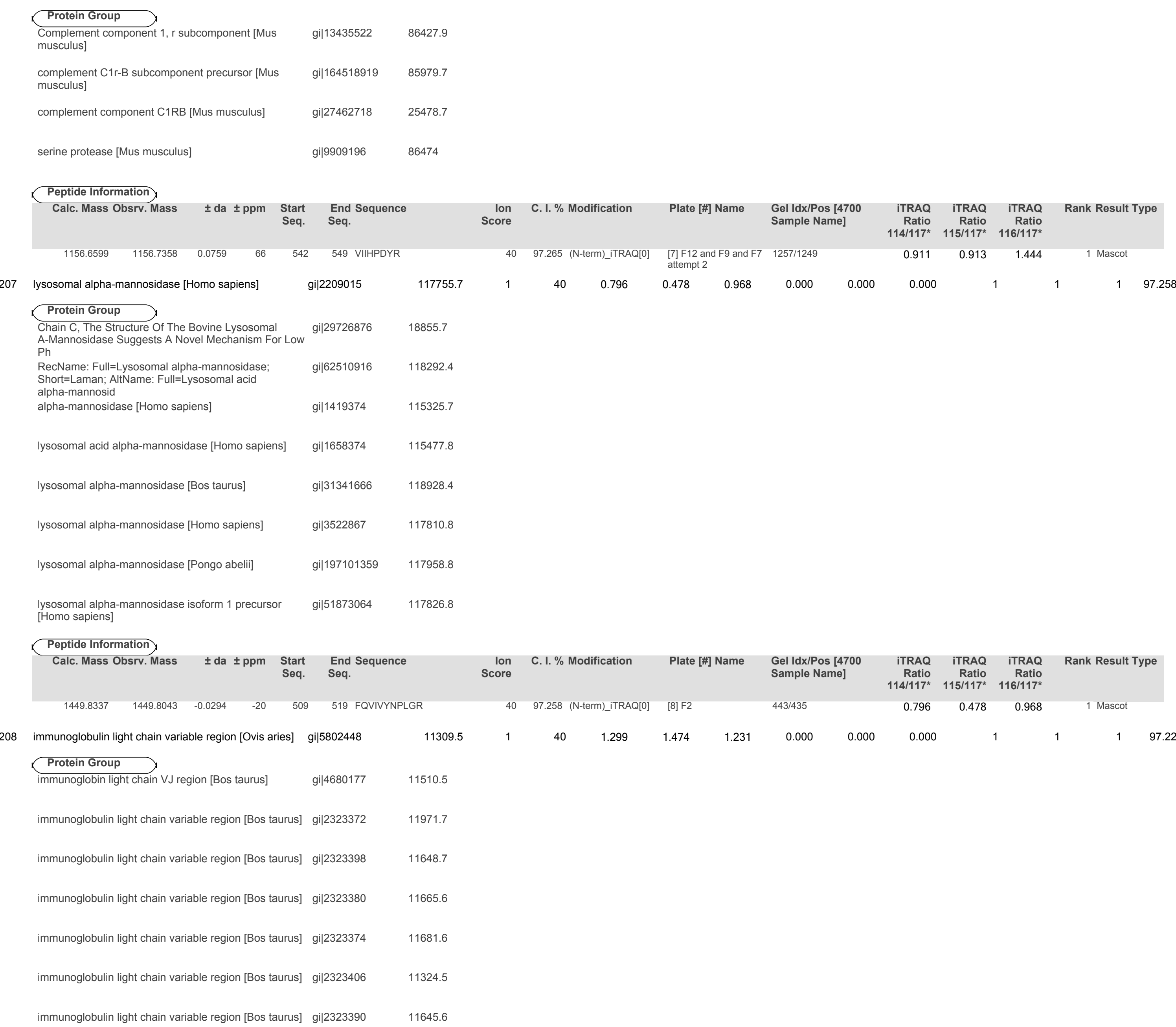


immunoglobulin light chain variable region [Bos taurus] gi|2323386

immunoglobulin light chain variable region [Bos taurus] gi|2323394

immunoglobulin light chain variable region [Bos taurus] gi|232338

immunoglobulin light chain variable region [Bos taurus] gi|2323404

immunoglobulin light chain variable region [Bos taurus] gi|2323396

immunoglobulin light chain variable region [Bos taurus] gi|2323402

immunoglobulin light chain variable region [Bos taurus] gi|232340

\section{Peptide Information}

\begin{tabular}{|c|c|c|c|c|c|c|c|c|c|c|c|}
\hline Calc. Mass Obsrv. Mass & \pm da $\pm p p m$ & $\begin{array}{l}\text { Start } \\
\text { Seq. }\end{array}$ & $\begin{array}{l}\text { End Sequence } \\
\text { Seq. }\end{array}$ & $\begin{array}{l}\text { lon } \\
\text { Score }\end{array}$ & C. I. \% Modification & Plate [\#] Name & $\begin{array}{l}\text { Gel Idx/Pos [4700 } \\
\text { Sample Name] }\end{array}$ & $\begin{array}{r}\text { iTRAQ } \\
\text { Ratio } \\
114 / 117^{*}\end{array}$ & $\begin{array}{r}\text { iTRAQ } \\
\text { Ratio } \\
115 / 117^{*}\end{array}$ & $\begin{array}{r}\text { iTRAQ } \\
\text { Ratio } \\
116 / 117^{*}\end{array}$ & Rank Result Type \\
\hline
\end{tabular}

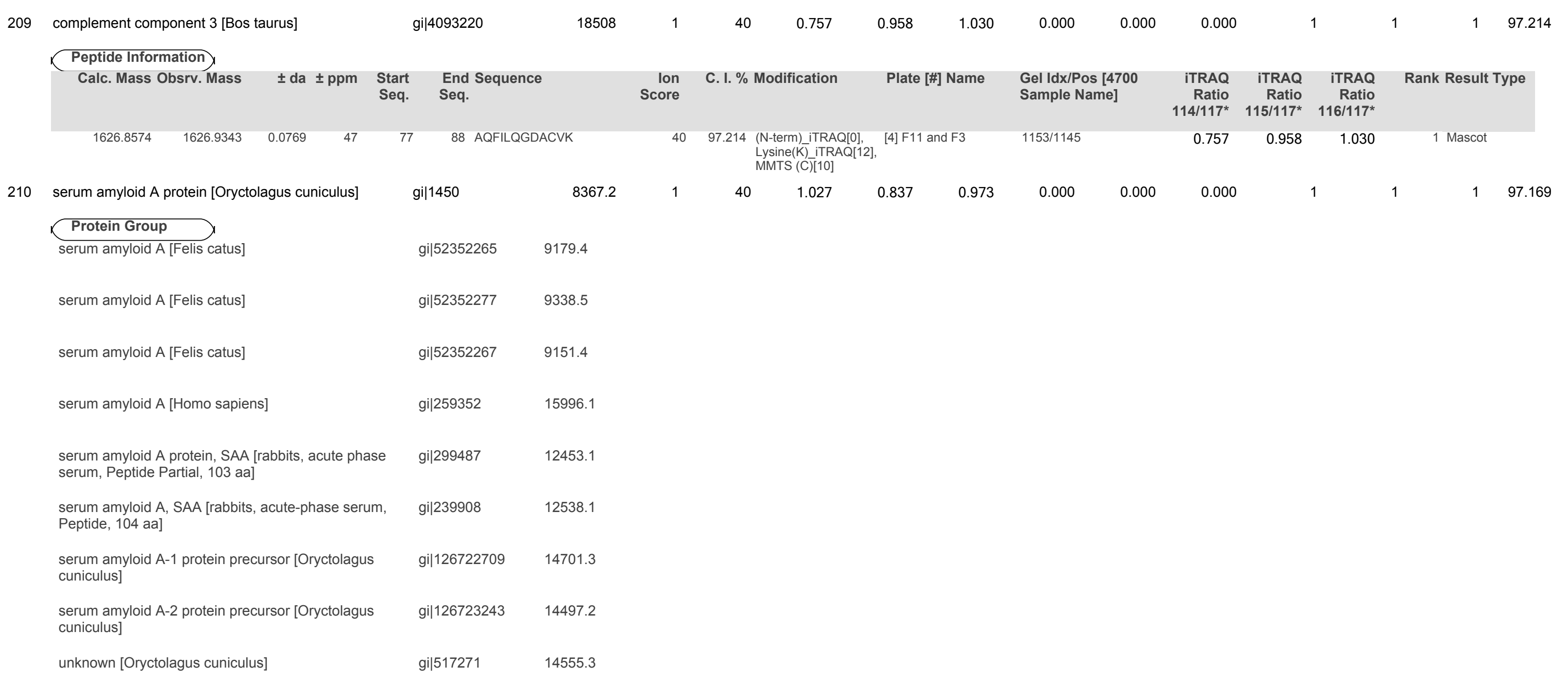




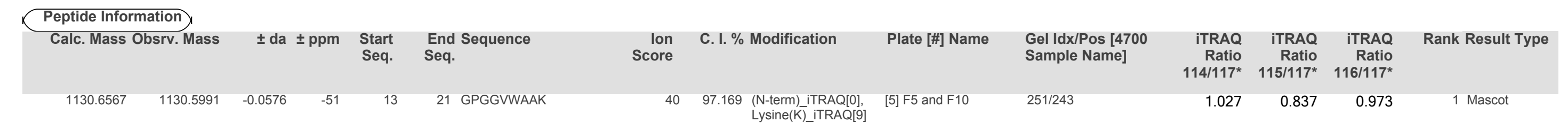

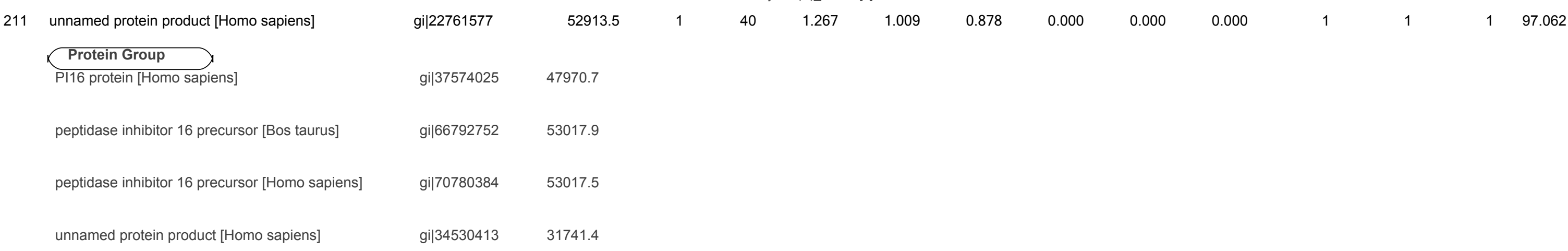




\section{Peptide Information}

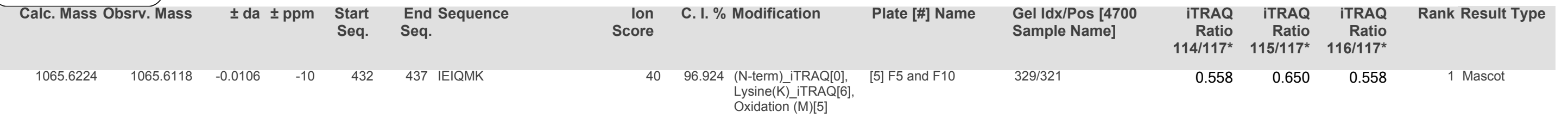

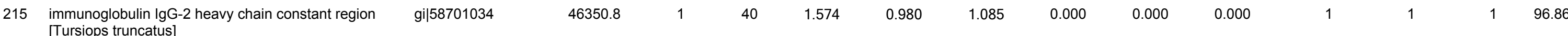

Protingoup

Protein Group
immunoglobulin IgG-1 heavy chain constant region
[Tursiops truncatus]

Peptide Information

\begin{tabular}{|c|c|c|c|c|c|c|c|c|c|c|c|}
\hline Calc. Mass Obsrv. Mass & $\pm \mathrm{da} \pm \mathrm{ppm}$ & $\begin{array}{l}\text { Start } \\
\text { Seq. }\end{array}$ & $\begin{array}{l}\text { End Sequence } \\
\text { Seq. }\end{array}$ & $\begin{array}{l}\text { Ion } \\
\text { Score }\end{array}$ & C. I. \% Modification & Plate [\#] Name & $\begin{array}{l}\text { Gel Idx/Pos [4700 } \\
\text { Sample Name] }\end{array}$ & $\begin{array}{r}\text { iTRAQ } \\
\text { Ratio } \\
114 / 117^{*}\end{array}$ & $\begin{array}{r}\text { iTRAQ } \\
\text { Ratio } \\
115 / 117^{*}\end{array}$ & $\begin{array}{r}\text { iTRAQ } \\
\text { Ratio } \\
116 / 117^{*}\end{array}$ & Rank Result Type \\
\hline
\end{tabular}

216 secreted nidogen domain protein precursor [Mus $\quad$ gil37605781 161194.4

nsculus]

Protein Group

FLJ00133 protein [Homo sapiens]

gi|18676472 147862

secreted protein SST3 [Mus musculus]

gi|29568116 $\quad 121217$

Peptide Information
Calc. Mass Obsrv. Mass
\pm da \pm ppm Start End Sequence
Ion C. I. \% Modification Plate [\#] Name Gel Idx/Pos [4700
$\begin{array}{llllll}1991.9828 & 1991.9224 & -0.0604 & -30 & 233 & 250 \\ & \text { TADMAEVETTTNVGVPG }\end{array}$
$307 / 299$

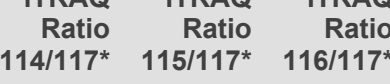

$39 \quad 96.711$ (N-term)_iTRAQ[0] $\quad[8] \mathrm{F} 2$

RecName: Full=Protein kinase C-binding protein
NELL2; AltName: Full=MEL91 protein; AltName:
Full=NE

gi|2494290 100048.6

Protein Group

gi|207079905 100063.5

RecName: Full=Protein kinase C-binding protein
NEL 2. AltName; Full=NEL-like protein 2; Flags: Prec

gi|2494291 99883.4

hypothetical protein [Pongo abelii]

gi|55727761 99972.6

mel [Mus musculus]

gi|1389827 $\quad 100387.7$

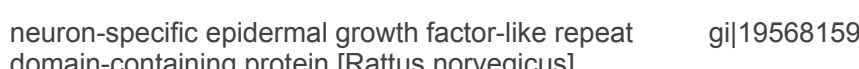

domain-containing protein [Rattus norvegicus]

protein kinase C-binding protein NELL2 [Mus musculus] gi|133922561

99751.4

100361.7

protein kinase C-binding protein NELL2 [Pongo abelii] gil197097656

100052.5

protein kinase C-binding protein NELL2 isoform b

precursor [Homo sapiens]

gi|5453766 $\quad 100276.7$

protein kinase C-binding protein NELL2 isoform C

gil223029474 100195.7

unnamed protein product [Mus musculus]

gi|26330366 96480

unnamed protein product [Mus musculus]

gi|26333475 $\quad 104708.8$ 
Peptide Information

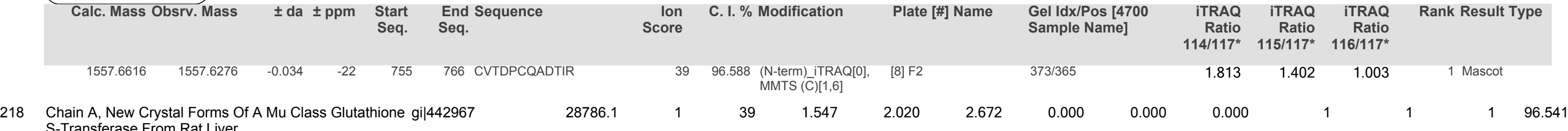

Chain A, Crystal Structures Of Class Mu Chimeric Gst gil|33356830 28707.1

Isoenzymes M1-2 And M2-1

Chain A, First-Sphere And Second-Sphere Electrostatic gil1943397

28770.1

Chain A, First-Sphere And Second-Sphere Electrostatic gil1943435
Effects In The Active Site Of A Class Mu Glut

28784.1

Chain A, First-Sphere And Second-Sphere Electrostatic gil1943433
Effects In The Active Site Of A Class Mu Glut

28772.1

Chain A, First-Sphere And Second-Sphere Electrostatic gi|1943431 28756.1

Chain A, Glutathione Transferase Mutant Y115f $\quad$ gi|29726512 28770.1

Chain A, Tetra-(5-Fluorotryptophanyl)-Glutathione $\quad$ gi|4388948 28485.8

Transferase

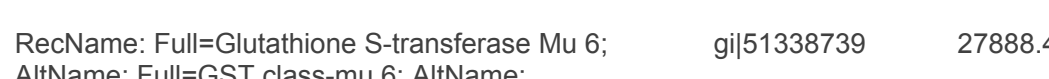

Full=Glutathio

glutathione S-transferase (EC 2.5.1.18) [Rattus $\quad$ gi|204501 28689

glutathione S-transferase Mu 1 [Rattus norvegicus] $\quad$ gi|l8393502 28917.1

glutathione S-transferase Mu 6 [Mus musculus] $\quad$ gil113680506 28192.6

glutathione S-transferase Yb-1 subunit (EC 2.5.1.18) $\quad$ gi|204503 28820.9

[Rattus norvegicus]

glutathione-S-transferase class M5 [Mus musculus] $\quad$ gi|2275021 11865

glutathione-S-transferase class M5 [Mus musculus] $\quad$ gi|2275019 27966.3

unnamed protein product [Mus musculus] $\quad$ gi|12853535 29718.2

\begin{tabular}{|c|c|c|c|c|c|c|c|c|c|c|c|}
\hline Calc. Mass Obsrv. Mass & \pm da $\pm \mathrm{ppm}$ & $\begin{array}{l}\text { Start } \\
\text { Seq. }\end{array}$ & $\begin{array}{l}\text { End Sequence } \\
\text { Seq. }\end{array}$ & $\begin{array}{l}\text { Ion } \\
\text { Score }\end{array}$ & C. I. \% Modification & Plate [\#] Name & $\begin{array}{l}\text { Gel Idx/Pos [4700 } \\
\text { Sample Name] }\end{array}$ & $\begin{array}{r}\text { iTRAQ } \\
\text { Ratio } \\
114 / 117^{*}\end{array}$ & $\begin{array}{r}\text { iTRAQ } \\
\text { Ratio } \\
115 / 117^{*}\end{array}$ & $\begin{array}{r}\text { iTRAQ } \\
\text { Ratio } \\
116 / 117^{*}\end{array}$ & Rank Result Type \\
\hline 1417.6178 & 0.1127 & 32 & 42 YAMGDAPDYDR & 39 & 96.541 (N-term)_iTRAQ[0] & [8] F2 & $238 / 230$ & 1.547 & 2.020 & 2.672 & 1 Mascot \\
\hline
\end{tabular}

75848.8

$\begin{array}{lll}39 & 0.230 & 0.342\end{array}$

0.780

$0.000 \quad 0.000$

1.547

1

1

Protein Group

gi|37956233 $\quad 75915.8$

RecName: Full=Fibrous sheath-interacting protein $1 \quad$ gil75048806 75358.6

fibrous sheath-interacting protein 1 [Homo sapiens] $\quad$ gi|116089284 $\quad 75860.7$

hypothetical protein [Macaca fascicularis]

gi|15207939 $\quad 60696.4$ 
220 Chain A, Crystal Structure of

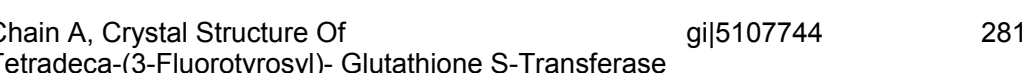
Peptide Information

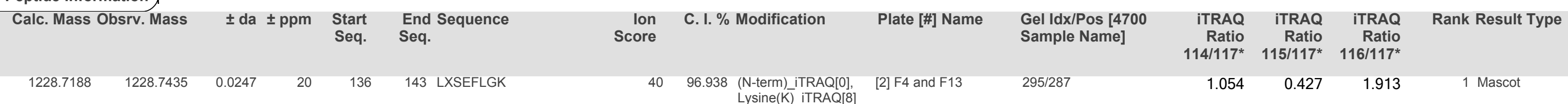

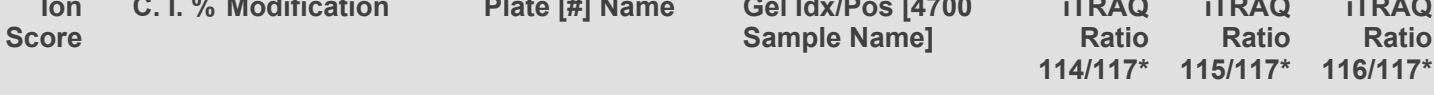

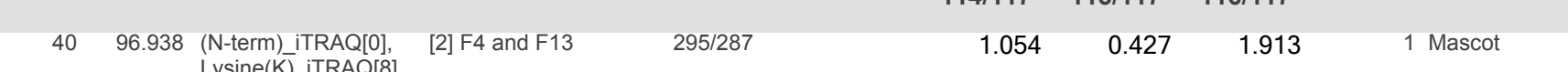

221 RecName: Full=EGF-containing fibulin-like extracellular gil9973149 matrix protein 2; AltName: Full=Fibulin-4;

Protein Group

EGF-containing fibulin-like extracellular matrix protein 2 gi|7634795

EGF-containing fibulin-like extracellular matrix protein 2 gi|54400722
[Rattus norvegicus]

EGF-containing fibulin-like extracellular matrix protein 2 gi|256355190 51854.1
isoform 1 [Mus musculus]

\section{Peptide Information}

\begin{tabular}{|c|c|c|c|c|c|c|c|c|c|c|c|}
\hline Calc. Mass Obsrv. Mass & \pm da $\pm p p m$ & $\begin{array}{l}\text { Start } \\
\text { Seq. }\end{array}$ & $\begin{array}{l}\text { End Sequence } \\
\text { Seq. }\end{array}$ & $\begin{array}{l}\text { Ion } \\
\text { Score }\end{array}$ & C. I. \% Modification & Plate [\#] Name & $\begin{array}{l}\text { Gel Idx/Pos [4700 } \\
\text { Sample Name] }\end{array}$ & $\begin{array}{r}\text { ITRAQ } \\
\text { Ratio } \\
114 / 117^{*}\end{array}$ & $\begin{array}{r}\text { iTRAQ } \\
\text { Ratio } \\
115 / 117^{*}\end{array}$ & $\begin{array}{r}\text { iTRAQ } \\
\text { Ratio } \\
116 / 117^{*}\end{array}$ & Rank Result Type \\
\hline
\end{tabular}

222 complement component C7 precursor [Homo sapiens] gi|45580688 103093.5 $\begin{array}{llllllllllll}51861.1 & 1 & 38 & 1.206 & 0.897 & 0.975 & 0.000 & 0.000 & 0.000 & 1 & 1 & 1\end{array}$ 51881.1 46862.

\begin{tabular}{|c|c|c|c|c|c|c|c|c|c|c|c|}
\hline Calc. Mass Obsrv. Mass & \pm da $\pm \mathrm{ppm}$ & $\begin{array}{l}\text { Start } \\
\text { Seq. }\end{array}$ & $\begin{array}{l}\text { End Sequence } \\
\text { Seq. }\end{array}$ & $\begin{array}{l}\text { lon } \\
\text { Score }\end{array}$ & C. I. \% Modification & Plate [\#] Name & $\begin{array}{l}\text { Gel Idx/Pos [4700 } \\
\text { Sample Name] }\end{array}$ & $\begin{array}{r}\text { iTRAQ } \\
\text { Ratio } \\
114 / 117^{*}\end{array}$ & $\begin{array}{r}\text { iTRAQ } \\
\text { Ratio } \\
115 / 117^{*}\end{array}$ & $\begin{array}{r}\text { iTRAQ } \\
\text { Ratio } \\
116 / 117^{*}\end{array}$ & Rank Result Type \\
\hline 1220.7338 & 0.0299 & 737 & 744 ILPLTVCK & 38 & 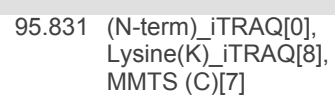 & [2] F4 and F13 & 285/277 & 0.985 & 0.978 & 1.044 & 1 Mascot \\
\hline
\end{tabular}

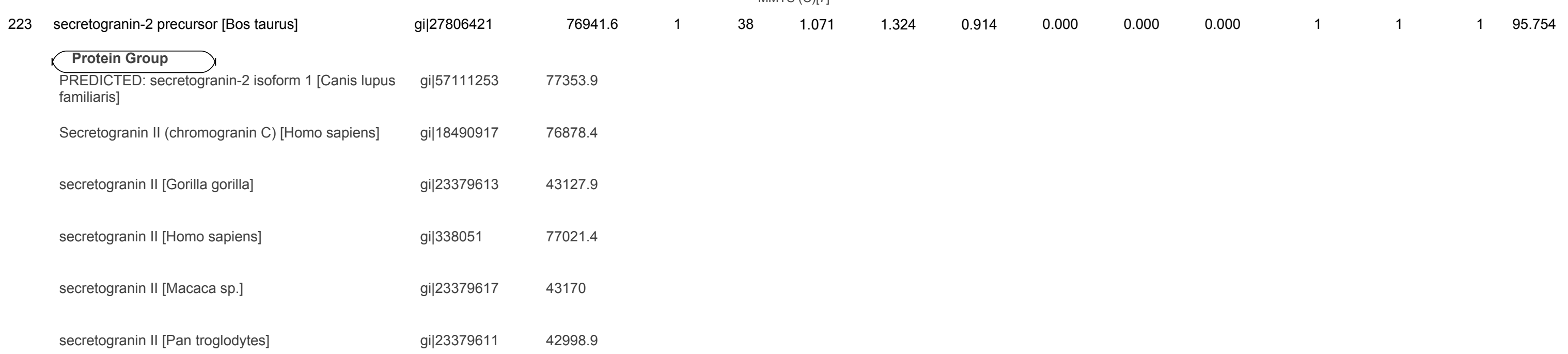


secretogranin II [Pongo pygmaeus]

secretogranin II [Saguinus oedipus]

secretogranin-2 precursor [Pongo abelii]

\section{Peptide Information}

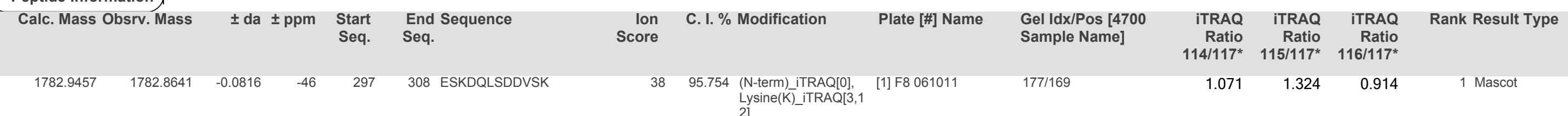

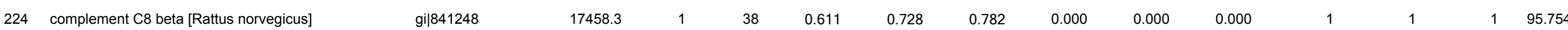
Peptide Information

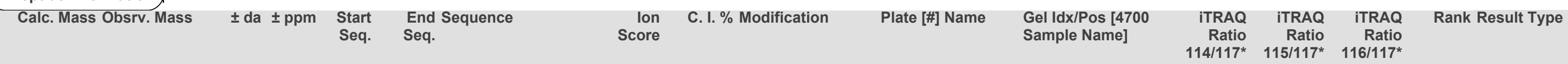

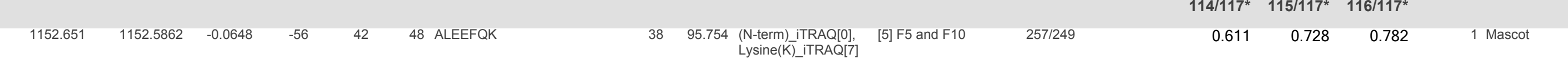

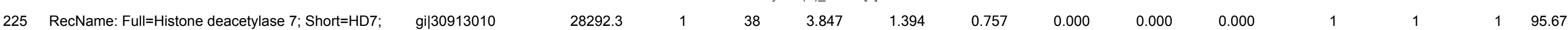

AltName: Full=Histone deacetylase $7 \mathrm{~A}$; Short=HD7a

Protein Group

RecName: Full=Histone deacetylase 7; Short=HD7;

AltName: Full=Histone deacetylase 7A; Short=HD7a

histone deacetylase 7 [Homo sapiens]

histone deacetylase 7 [Mus musculus]

gi|7542540 $\quad 97325.3$

histone deacetylase 7 isoform 3 [Mus musculus]

gil6911184 $\quad 106700.9$

histone deacetylase 7 isoform 4 [Mus musculus]

gi|324073138 107291.5

histone deacetylase 7 isoform a [Homo sapiens]

gil40254548 $\quad 106668.9$

histone deacetylase 7 isoform d [Homo sapiens]

gil169234807 $\quad 112020.7$

histone deacetylase 7A variant 3 [Homo sapiens]

gi|148539870 $\quad 108188.4$

hypothetical protein [Homo sapiens]

gil32482808 $\quad 104239.9$

mFLJ00062 protein [Mus musculus]

gi|5911907 $\quad 99192.4$

unnamed protein product [Mus musculus]

gi|47847402 $\quad 95956.4$

unnamed protein product [Mus musculus]

gi|26326835 $\quad 108247.2$

gi|26331526 $\quad 107367.6$

$\begin{array}{ll}\text { unnamed protein product [Mus musculus] } & \text { gil26354072 }\end{array}$

unnamed protein product [Mus musculus]

gi|26353936 $\quad 101963.6$

Peptide Information

\begin{tabular}{|c|c|c|c|c|c|c|c|c|c|c|c|}
\hline Calc. Mass Obsrv. Mass & $\pm \mathrm{da} \pm \mathrm{ppm}$ & $\begin{array}{l}\text { Start } \\
\text { Seq. }\end{array}$ & $\begin{array}{l}\text { End Sequence } \\
\text { Seq. }\end{array}$ & $\begin{array}{l}\text { lon } \\
\text { Score }\end{array}$ & C. I. \% Modification & Plate [\#] Name & $\begin{array}{l}\text { Gel Idx/Pos [4700 } \\
\text { Sample Name] }\end{array}$ & $\begin{array}{r}\text { iTRAQ } \\
\text { Ratio } \\
114 / 117^{*}\end{array}$ & $\begin{array}{r}\text { iTRAQ } \\
\text { Ratio } \\
115 / 117^{*}\end{array}$ & $\begin{array}{r}\text { iTRAQ } \\
\text { Ratio } \\
11 / 117^{*}\end{array}$ & Rank Result Type \\
\hline
\end{tabular}


$\begin{array}{llllll}1380.8586 & 1380.7924 & -0.0662 & -48 & 1 & 9 \text { VASYGVKPK }\end{array}$

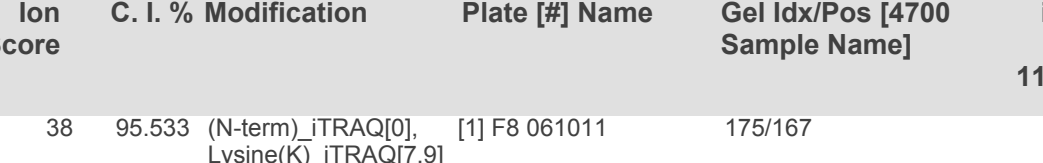

$\begin{array}{rrr}114 / 117^{*} & 115 / 117^{*} & \begin{array}{r}\text { Ratio } \\ 116 / 117^{*}\end{array} \\ 11228 & 1.177 & 129\end{array}$

$227 \begin{aligned} & \text { nuclear factor of activated T-cells, cytoplasmic } 2 \text { isoform gi|27886541 } \\ & \text { C [Homo sapiens] }\end{aligned}$

105862.5

Chain L, Structure of Nfat1 Bound As A Dimer To The gil37927427
Hiv-1 Ltr Kb Element

Chain M, Crystal Structure Of Human Nfat1 Bound $\quad$ gil46015057

35436.7

Chain N, Structure Of The Dna Binding Domains Of $\quad$ gi|3212242

NFAT1-A [Mus musculus]

gi|1353237

37211.5

Nfatc2 protein [Mus musculus]

gi|39793877 $\quad 70671.8$

T cell transcription factor NFAT1 isoform B [Mus
musculus]

gi|1353399

T cell transcription factor NFAT1 isoform C [Mus
musculus]

gi|1353401

nuclear factor of activated T-cells, cytoplasmic 2 isoform gi|27886539

nuclear factor of activated T-cells, cytoplasmic 2 isoform gi|81295412
c [Mus musculus]

transcription factor NFAT [Sus scrofa] g gi|3335546

transcription factor NFAT1 isoform B [Homo sapiens] gil1353774

transcription factor NFAT1 isoform C [Homo sapiens] gi|1353776

$\begin{array}{lllll}38 & 0.961 & 1.561 & 0.377 & 0.000\end{array}$

1.228

$1.177 \quad 1.292 \quad 1$ Mascot
1

36.7

35262.7

121352

105114

105635.2

105357.3

52075.1

14988.2

105375.2

105880.5

transcription factor NFAT1-D [Mus musculus] $\quad$ gil13936689 $\quad 75714.4$

\section{Peptide Information}

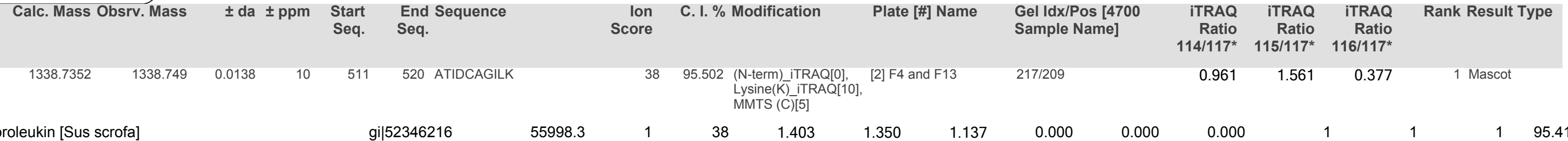

228 fibroleukin [Sus scrofa]

gi|52346216 $\quad 55998.3$

$137 \quad 0.000$

Protein Group protein [Homo sapien

gi|17389565 $\quad 18653.7$

fibroleukin precursor [Homo sapiens]

gil5730075

55890.4

Peptide Information

$\begin{array}{lll}\text { Calc. Mass Obsrv. Mass } \quad \pm \text { da } \pm \text { ppm } & \begin{array}{l}\text { Start } \\ \text { Seq. }\end{array} & \begin{array}{l}\text { End Sequence } \\ \text { Seq. }\end{array}\end{array}$

Ion C. I. \% Modification Plate [\#] Name Gel Idx/Pos [4700

ample Name]

ITRAQ ITRAQ iTRAQ Rank Result Type $\begin{array}{rrr}\text { Ratio } & \text { Ratio } & \text { Ratio } \\ 114 / 117^{*} & 115 / 117^{*} & 116 / 117^{*} \\ 11403 & 1350 & 1.137\end{array}$

$\begin{array}{lll}1.403 & 1.350 & 1.137\end{array}$

$\left.38 \quad 95.419 \begin{array}{c}\text { (N-term) iTRAQ[0]] } \\ \text { Lysine(K) iTRAQ[7,8] }\end{array}\right]$ [1] F8 $061011 \quad 215 / 207$

0.000 


\begin{tabular}{|c|c|c|}
\hline Protein Group & gi|37182914 & 52718.6 \\
\hline UP50 [Homo sapiens] & gi|3676822 & 52939.7 \\
\hline $\begin{array}{l}\text { embryonic vascular EGF repeat-containing protein } \\
\text { EVEC [Rattus norvegicus] }\end{array}$ & gi|4583509 & 52670.6 \\
\hline fibulin- 5 precursor [Bos taurus] & gil62460592 2010 & 52736.5 \\
\hline fibulin-5 precursor [Homo sapiens] & gi|19743803 & 52752.6 \\
\hline fibulin-5 precursor [Mus musculus] & gi|6753824 & 52719.5 \\
\hline fibulin-5 precursor [Pongo abelii] & gi|197102314 & 52851.7 \\
\hline in produc & gi|28207929 & 62962.5 \\
\hline
\end{tabular}

\begin{tabular}{|c|c|c|c|c|c|c|c|c|c|c|c|}
\hline Calc. Mass Obsrv. Mass & \pm da \pm ppm & $\begin{array}{l}\text { Start } \\
\text { Seq. }\end{array}$ & $\begin{array}{l}\text { End Sequence } \\
\text { Seq. }\end{array}$ & $\begin{array}{l}\text { Ion } \\
\text { Score }\end{array}$ & C. I. \% Modification & Plate [\#] Name & $\begin{array}{l}\text { Gel Idx/Pos [4700 } \\
\text { Sample Name] }\end{array}$ & $\begin{array}{r}\text { iTRAQ } \\
\text { Ratio } \\
114 / 117^{*}\end{array}$ & $\begin{array}{r}\text { iTRAQ } \\
\text { Ratio } \\
115 / 117^{*}\end{array}$ & $\begin{array}{r}\text { iTRAQ } \\
\text { Ratio } \\
116 / 117^{*}\end{array}$ & Rank Result Type \\
\hline 1708.8511 & -0.0301 & 360 & 373 SVPADIFQMQATTR & $38>$ & 95.376 (N-term)_iTRAQ[0] & [8] F2 & 393/385 & 1.296 & 1.182 & 1.122 & 1 Mascot \\
\hline
\end{tabular}

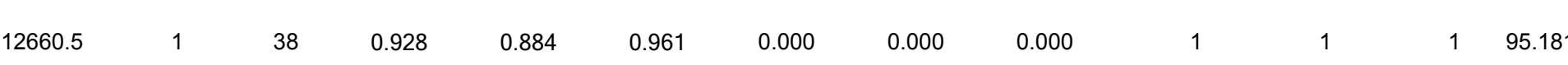

Protein Goup

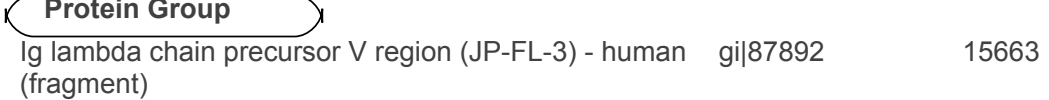

immunoglobulin lambda light chain variable region $\quad$ gil4324216 $\quad 12229.1$

[Homo sapiens]

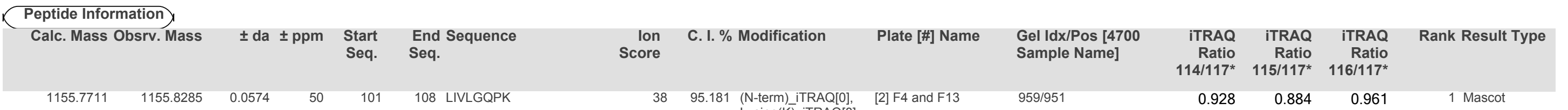

231 precursor polypeptide (AA -19 to 206) [Mus musculus] gi|50564

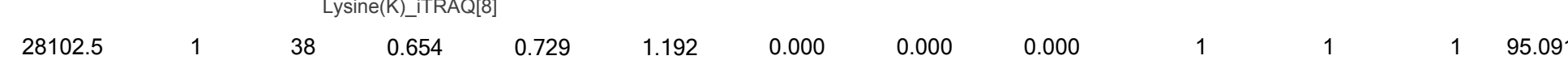

C-reactive protein [Homo sapiens]

27390.2

C-reactive protein [Oryctolagus cuniculus

gi|30224

27390.2

C-reactive protein precursor [Homo sapiens] $\quad$ gi|l55770842 $\quad 27322.2$

C-reactive protein precursor [Oryctolagus cuniculus] $\quad$ gil126722616 $\quad 27904.3$

$\begin{array}{ll}\text { C-reactive protein precursor [Sus scrofa] } & \text { gil55742770 }\end{array} \quad 26821.7$

C-reactive protein, pentraxin-related [Homo sapiens] $\quad$ gi|55665343 $\quad 13012.9$

$\begin{array}{lll}\text { CRP protein [Homo sapiens] } & \text { gil18088467 } & 11797.3\end{array}$

Chain A, Human C-Reactive Protein gi|1942435 25142

RecName: Full=C-reactive protein; Flags: Precursor $\quad$ gi|117487 28076.5 
Peptide Information

Calc. Mass Obsrv. Mass $\quad \pm$ da \pm ppm Start End Sequence

$\begin{array}{llllll}996.6127 & 996.5723 & -0.0404 & -41 & 27 & 32 \text { AFVFPK }\end{array}$

lon
Score

C. I. \% Modification

Plate [\#] Name

Gel Idx/Pos [4700
Sample Name]

Sample Name]

$38 \quad 95.091 \begin{aligned} & \left(\begin{array}{l}\text { N-term)_TRAQ[0], } \\ \text { Lysine(K)_TRRA[6] }\end{array}\right. \\ & {[5] \text { F5 and F10 }}\end{aligned}$ $\begin{array}{rrr}\text { iTRAQ } & \text { iTRAQ } & \text { iTRA } \\ \text { Ratio } & \text { Ratio } \\ \text { Ratio }\end{array}$ $0.654 \quad 0.729$
Rank Result Type 